\title{
Toroidal Dehn fillings on hyperbolic 3-manifolds
}

\author{
Cameron McA. Gordon ${ }^{1}$ and Ying-Qing $\mathrm{Wu}^{2}$
}

\begin{abstract}
We determine all hyperbolic 3 -manifolds $M$ admitting two toroidal Dehn fillings at distance 4 or 5 . We show that if $M$ is a hyperbolic 3manifold with a torus boundary component $T_{0}$, and $r, s$ are two slopes on $T_{0}$ with $\Delta(r, s)=4$ or 5 such that $M(r)$ and $M(s)$ both contain an essential torus, then $M$ is either one of 14 specific manifolds $M_{i}$, or obtained from $M_{1}, M_{2}, M_{3}$ or $M_{14}$ by attaching a solid torus to $\partial M_{i}-T_{0}$. All the manifolds $M_{i}$ are hyperbolic, and we show that only the first three can be embedded into $S^{3}$. As a consequence, this leads to a complete classification of all hyperbolic knots in $S^{3}$ admitting two toroidal surgeries with distance at least 4 .
\end{abstract}

\section{Introduction}

Let $M$ be a hyperbolic 3-manifold, by which we shall mean a compact, connected, orientable 3-manifold such that $M$ with its boundary tori removed admits a complete hyperbolic structure with totally geodesic boundary, and suppose that $M$ has a torus boundary component $T_{0}$. If $r$ is a slope on $T_{0}$ then $M(r)$ will denote the 3-manifold obtained by $r$-Dehn filling on $M$, i.e. attaching a solid torus $V_{r}$ to $M$ along $T_{0}$ in such a way that $r$ bounds a disk in $V_{r}$. The Dehn filling $M(r)$ and the slope $r$ are said to be exceptional if $M(r)$ is either reducible, $\partial$-reducible, annular, toroidal, or a small Seifert fiber space. Modulo the Geometrization Conjecture, the manifold $M(r)$ is hyperbolic if and only if $M(r)$ is not exceptional.

Thurston's Hyperbolic Dehn Surgery Theorem asserts that there are only finitely many exceptional Dehn fillings on each torus boundary component of $M$. It is known that if $r, s$ are both exceptional then the geometric intersection number $\Delta=\Delta(r, s)$, also known as the distance between $r$ and $s$, is small. In fact, the least upper bounds for $\Delta$ have been determined for all cases where neither $M(r)$ nor $M(s)$ is a small Seifert fiber space, by the work of many people. See [GW2] and the references therein.

\footnotetext{
${ }^{0}$ Mathematics subject classification (1991): Primary $57 N 10$.

${ }^{0}$ Keywords and phrases: Toroidal manifolds, Dehn fillings

${ }^{1}$ Partially supported by NSF grant DMS 0305846.

${ }^{2}$ Partially supported by NSF grant DMS 0203394.
} 
For toroidal fillings, it was shown by Gordon [Go] that if $r, s$ are toroidal slopes then $\Delta(r, s) \leq 8$, and moreover there are exactly two manifolds $M$ with $\Delta=8$, one with $\Delta=7$, and one with $\Delta=6$. In this paper we classify all the hyperbolic 3-manifolds which admit two toroidal Dehn fillings with $\Delta=4$ or 5 .

Already when $\Delta=5$ there are infinitely many such manifolds. To see this, let $M$ be the exterior of the Whitehead sister link, also known as the $(-2,3,8)$-pretzel link. The boundary of $M$ consists of two tori $T_{0}$ and $T_{1}$, and there are slopes $r, s$ on $T_{0}$ with $\Delta(r, s)=5$ such that the Dehn filled manifolds $M(r)=M(r, *), M(s)=M(s, *)$ are toroidal; see for example [GW3]. Now for infinitely many slopes $t$ on $T_{1}, M_{t}=M(*, t)$ will be hyperbolic and $M_{t}(r)=M(r)(t), M_{t}(s)=M(s)(t)$ will be toroidal. In this way we get infinitely many hyperbolic 3 -manifolds with boundary a single torus having two toroidal fillings at distance 5 . We shall show that, modulo this phenomenon, there are only finitely many $M$ with two toroidal fillings at distance 4 or 5 , and explicitly identify them. Define two triples $\left(N_{1}, r_{1}, s_{1}\right)$ and $\left(N_{2}, r_{2}, s_{2}\right)$ to be equivalent, denoted by $\left(N_{1}, r_{1}, s_{1}\right) \cong\left(N_{2}, r_{2}, s_{2}\right)$, if there is a homeomorphism from $N_{1}$ to $N_{2}$ which sends the boundary slopes $\left(r_{1}, s_{1}\right)$ to $\left(r_{2}, s_{2}\right)$ or $\left(s_{2}, r_{2}\right)$.

Theorem 1.1 There exist 14 3-manifolds $M_{i}, 1 \leq i \leq 14$, such that

(1) $M_{i}$ is hyperbolic, $1 \leq i \leq 14$;

(2) $\partial M_{i}$ consists of two tori $T_{0}, T_{1}$ if $i \in\{1,2,3,14\}$, and a single torus $T_{0}$ otherwise;

(3) there are slopes $r_{i}, s_{i}$ on the boundary component $T_{0}$ of $M_{i}$ such that $M\left(r_{i}\right)$ and $M\left(s_{i}\right)$ are toroidal, where $\Delta\left(r_{i}, s_{i}\right)=4$ if $i \in\{1,2,4,6,9,13,14\}$, and $\Delta\left(r_{i}, s_{i}\right)=5$ if $i \in\{3,5,7,8,10,11,12\}$;

(4) if $M$ is a hyperbolic 3-manifold with toroidal Dehn fillings $M(r), M(s)$ where $\Delta(r, s)=4$ or 5 , then $(M, r, s)$ is equivalent either to $\left(M_{i}, r_{i}, s_{i}\right)$ for some $1 \leq i \leq 14$, or to $\left(M_{i}(t), r_{i}, s_{i}\right)$ where $i \in\{1,2,3,14\}$ and $t$ is a slope on the boundary component $T_{1}$ of $M_{i}$.

Proof. The manifolds $M_{i}$ are defined in Definition 21.3. (1) is Theorem 23.14. (2) follows from the definition. (3) and (4) follow from Theorem 21.4.

Remark 1.2 Part (4) in Theorem 1.1 is still true if the hyperbolicity is replaced by the assumption that $M$ is compact, connected, orientable, irreducible, atoroidal, and non Seifert fibered, in other words, $M$ may be annular or $\partial$ reducible but not Seifert fibered.

Proof. First assume $M$ is $\partial$-irreducible. Then any essential annulus must have at least one boundary component on a non-toroidal boundary component of $M$ as otherwise $M$ would be either toroidal or Seifert fibered. Attaching a hyperbolic manifold $X$ to each non-toroidal boundary component of $M$ will produce a hyperbolic manifold $M^{\prime}$, and we may choose $X$ so that $M^{\prime}$ has more than three boundary components. One can show that the $M^{\prime}\left(r_{i}\right)$ are still toroidal for $i=1,2$, which is a contradiction to Theorem 21.4. Now assume $M$ is $\partial$ reducible. Then $M$ is obtained by attaching 1-handles to the boundary of a 
manifold $M^{\prime \prime}$. If a 1-handle is attached to a toroidal component $T$ of $\partial M^{\prime \prime}$ then either $M^{\prime \prime}=T \times I$, which is impossible because $M\left(r_{i}\right)$ would be a handlebody and hence atoroidal, or $T$ would be an essential torus in $M$, contradicting the assumption. It follows that $M^{\prime \prime}$ has a higher genus boundary component. One can check that the $M^{\prime \prime}\left(r_{i}\right)$ are still toroidal, which leads to a contradiction to Theorem 21.4 as above.

The manifolds $M_{1}, M_{2}$ and $M_{3}$ were discussed in [GW1]; $M_{i}, i=1,2,3$ is the exterior of a link $L_{i}$ in $S^{3}$, where $L_{1}$ is the Whitehead link, $L_{2}$ is the 2bridge link associated to the rational number $3 / 10$, and $L_{3}$ is the Whitehead sister link. See Figure 24.1. The other $M_{i}$ can be built using intersection graphs on tori, see Definition 21.3 for more details. For $i \neq 4,5$, each $M_{i}$ can also be described as a double branched cover of a tangle $Q_{i}=\left(W_{i}, K_{i}\right)$, where $W_{i}$ is a 3 -ball for $i=6, \ldots, 13$, and a once punctured 3-ball for $i=1,2,3,14$. This is done in [GW1] for $i=1,2,3$, and in Section 22 for the other cases. See Lemma 22.2 .

Some results on the case $\Delta=5$ have been independently obtained by Teragaito [T2]. He obtains a finite set of pairs of intersection graphs of punctured tori at distance 5 which must contain all the pairs of graphs that arise from two toroidal fillings on a hyperbolic 3-manifold at distance 5. One of his pairs produces a non-hyperbolic manifold while the others correspond to the manifolds in our list for $\Delta=5$.

We remark that the related problem of determining all hyperbolic 3-manifolds with two Dehn fillings at distance at least 4 that yield manifolds containing Klein bottles has been solved by Lee [L1, L2] (see also [MaS]).

Since $M_{i}$ has more than one boundary component only when $i \in\{1,2,3,14\}$, we have the following corollary to Theorem 1.1 (together with [Go]), which in the case $\Delta=5$ is due to Lee [L1]. Note that all boundary components of the manifolds are tori.

Corollary 1.3 Let $M$ be a hyperbolic 3-manifold with more than one boundary component, having toroidal Dehn fillings $M(r), M(s)$ with $\Delta=\Delta(r, s) \geq 4$. Then each boundary component of $M$ is a torus, and either

(1) $\Delta=4$ and $(M, r, s) \cong\left(M_{i}, r_{i}, s_{i}\right)$ for $i \in\{1,2,14\}$, or

(2) $\Delta=5$ and $(M, r, s) \cong\left(M_{3}, r_{3}, s_{3}\right)$.

In [GW1] and [GW3] it is shown that if $M$ is a hyperbolic 3-manifold with fillings $M(r)$ and $M(s)$, one of which is annular and the other either toroidal or annular, then either $(M, r, s) \cong\left(M_{i}, r_{i}, s_{i}\right)$ for $i \in\{1,2,3\}$, or $\Delta(r, s) \leq 3$. It is also known that if $M(r)$ contains an essential sphere or disk, and $M(s)$ contains an essential sphere, disk, annulus or torus, then $\Delta(r, s) \leq 3$; see [GW2] and the references listed there. Corollary 1.3 then gives

Corollary 1.4 Let $M$ be a hyperbolic 3-manifold with a torus boundary component $T_{0}$ and at least one other boundary component. Let $r, s$ be exceptional slopes on $T_{0}$. Then either $(M, r, s) \cong\left(M_{i}, r_{i}, s_{i}\right)$ for $i \in\{1,2,3,14\}$, or $\Delta(r, s) \leq 3$. 
A pair $\left(M, T_{0}\right)$ is called a large manifold if $T_{0}$ is a torus on the boundary of the 3-manifold $M$ and $H_{2}\left(M, \partial M-T_{0}\right) \neq 0$ (see [Wu3]). Teragaito [T2] proved that there is no large hyperbolic manifold $M$ admitting two toroidal fillings of distance at least 5 . The following corollary clarifies the case of distance 4 .

Theorem 22.3 Suppose $\left(M, T_{0}\right)$ is a large manifold and $M$ is hyperbolic and contains two toroidal slopes $r_{1}, r_{2}$ on $T_{0}$ with $\Delta\left(r_{1}, r_{2}\right) \geq 4$. Then $M$ is the Whitehead link exterior, and $\Delta\left(r_{1}, r_{2}\right)=4$.

Theorem 1.1 gives information about toroidal Dehn surgeries on hyperbolic knots in $S^{3}$. It follows from [Go] that the only such knot with two toroidal surgeries at distance $>5$ is the figure eight knot, for which the 4 and -4 surgeries are toroidal. Teragaito has shown [T1] that the only hyperbolic knots with two toroidal surgeries at distance 5 are the Eudave-Muñoz knots $k(2,-1, n, 0)$, $n \neq 1$. We can now determine the knots with toroidal surgeries at distance 4 . Denote by $L_{i}=K_{i}^{\prime} \cup K_{i}^{\prime \prime}$ the link in Figure 24.1(i), where $K_{i}^{\prime}$ is the component on the left. Denote by $L_{i}(n)$ the knot obtained from $K_{i}^{\prime \prime}$ by $1 / n$ surgery on $K_{i}^{\prime}$. One can check that $L_{3}(n)$ is the same as the Eudave-Muñoz knot $k(3,1,-n, 0)$ in [Eu, Figure 25], which is the mirror-image of $k(2,-1,1+n, 0)$ [Eu, Proposition 1.4].

Theorem 24.4 Suppose $K$ is a hyperbolic knot in $S^{3}$ admitting two toroidal surgeries $K\left(r_{1}\right), K\left(r_{2}\right)$ with $\Delta\left(r_{1}, r_{2}\right) \geq 4$. Then $\left(K, r_{1}, r_{2}\right)$ is equivalent to one of the following, where $n$ is an integer.

(1) $K=L_{1}(n), r_{1}=0, r_{2}=4$.

(2) $K=L_{2}(n), r_{1}=2-9 n, r_{2}=-2-9 n$.

(3) $K=L_{3}(n), r_{1}=-9-25 n, r_{2}=-(13 / 2)-25 n$.

(4) $K$ is the Figure 8 knot, $r_{1}=4, r_{2}=-4$.

The only hyperbolic knots known to have more than two toroidal surgeries are the figure eight knot and the $(-2,3,7)$-pretzel knot, with toroidal slopes $\{-4,0,4\}$ and $\{16,37 / 2,20\}$ respectively. This led Eudave-Muñoz [Eu] to conjecture that a hyperbolic knot in $S^{3}$ has at most three toroidal surgeries. Teragaito [T1] showed that there can be at most five toroidal surgeries. Theorem 1.1 and [T1, Corollary 1.2] lead to the following improvement.

Corollary 24.5 A hyperbolic knot in $S^{3}$ has at most four toroidal surgeries. If there are four, then they are consecutive integers.

Here is a sketch of the proof of Theorem 1.1. A toroidal Dehn filling $M(r)$ on a hyperbolic 3-manifold $M$ gives rise to an essential punctured torus $F$ in $M$ whose boundary consists of $n>0$ circles of slope $r$ on $T_{0}$, where the capped-off surface $\hat{F}$ is an essential torus in $M(r)$. Hence, in the usual way (see Section 2 ), two toroidal fillings $M\left(r_{1}\right), M\left(r_{2}\right)$ give rise to a pair of intersection graphs $\Gamma_{1}, \Gamma_{2}$ on the tori $\hat{F}_{1}, \hat{F}_{2}$, with $n_{1}, n_{2}$ vertices respectively. The proof consists of a detailed analysis of the possible pairs of intersection graphs with $\Delta\left(r_{1}, r_{2}\right)=4$ 
or 5, using Scharlemann cycles and other tools developed in earlier works in this area. This enables us to eliminate all but 17 pairs of graphs. As is usual in this kind of setting, the permissible graphs all have small numbers of vertices. Eleven of the pairs correspond to the manifolds $M_{i}, 4 \leq i \leq 14$. We show that any of the remaining pairs must correspond to a pair of fillings on $M_{i}$ or $M_{i}(t)$ for $i \in\{1,2,3\}$.

Here is a more detailed summary of the organization of the paper. Section 2 contains the basic definitions and some preliminary lemmas. In Sections 3-5 we deal with the generic case $n_{1}, n_{2}>4$, ultimately showing (Proposition 5.11) that this case cannot occur. More specifically, Section 3 shows that the reduced positive graph $\hat{\Gamma}_{a}^{+}$of $\Gamma_{a}$ (see Section 2 for definitions) has no interior vertices, and this is strengthened in Section 4 to showing that each component of $\hat{\Gamma}_{a}^{+}$ must be one of the 11 graphs in Figure 4.2. These are ruled out one by one in Section 5. In Sections 6-11 we consider the case where some $n_{a}=4$. Section 6 discusses the situation where the graph $\Gamma_{a}$ is kleinian; this arises when the torus $\hat{F}_{a}$ is the boundary of a regular neighborhood of a Klein bottle in $M\left(r_{a}\right)$. (The results here are also used in the discussion of the case $n_{1}, n_{2} \leq 2$.) Sections 7 , 8 and 9 show that if $n_{a}=4$ and $\Gamma_{b}$ is non-positive then $n_{b} \leq 4$. Section 10 shows that if $\Gamma_{1}$ and $\Gamma_{2}$ are both non-positive then $n_{1}=n_{2}=4$ is impossible. Section 11 shows (Proposition 11.9) that if $\Gamma_{b}$ is positive then there are exactly two pairs of graphs, one with $n_{b}=2$, the other with $n_{b}=1$. These give the manifolds $M_{4}$ and $M_{5}$ respectively. If we suppose $n_{a} \leq n_{b}$, it now easily follows (Proposition 11.10) that $n_{a} \leq 2$.

In Sections 12-16 we deal with the case $n_{a} \leq 2, n_{b} \geq 3$. The conclusion (Proposition 16.8) is that here there are exactly six pairs of graphs. Two of these are the ones described in Section 11, and the four new pairs give the manifolds $M_{6}, M_{7}, M_{8}$ and $M_{9}$. More precisely, in Section 12 we rule out the case where $\Gamma_{b}$ is positive, and in Sections 13 and 14 we consider the case where $n_{b}>4$ and both graphs $\Gamma_{1}$ and $\Gamma_{2}$ are non-positive. It turns out that here there is exactly one pair of graphs (Proposition 14.7), corresponding to the manifold $M_{6}$. We may now assume that $n_{b}=3$ or 4 . Section 15 establishes some notation and elementary properties for graphs with $n_{a} \leq 2$. In Section 16 we show that if $\Gamma_{1}$ and $\Gamma_{2}$ are non-positive then $n_{b}=3$ is impossible and if $n_{b}=4$ then there are exactly three examples, $M_{7}, M_{8}$ and $M_{9}$.

Sections 17-20 deal with the remaining cases where both $n_{1}$ and $n_{2}$ are $\leq 2$. In Section 17 we introduce an equivalence relation, equidistance, on the set of edges of a graph $\Gamma_{a}$, and show that, under the natural bijection between the edges of $\Gamma_{1}$ and $\Gamma_{2}$, the two graphs induce the same equivalence relation. This gives a convenient way of ruling out certain pairs of graphs. Section 18 considers the case $n_{a}=2$ and $n_{b}=1$, and shows that here there are exactly three examples. Section 19 considers the case $n_{1}=n_{2}=2, \Gamma_{b}$ positive, showing that there are two examples. Finally, in Section 20 we consider the case $n_{1}=$ $n_{2}=2, \Gamma_{1}$ and $\Gamma_{2}$ both non-positive, and show that there are exactly six pairs of graphs in this case. The final list of all 11 possible pairs of graphs with $n_{1}, n_{2} \leq 2$ is given in Proposition 20.4. Five of these correspond to the manifolds $M_{10}, M_{11}, M_{12}, M_{13}$ and $M_{14}$. 
The remaining six pairs of graphs in Proposition 20.4 have the property that one of the graphs has a non-disk face. In Section 21 we show (Lemma 21.2), using the classification of toroidal/annular and annular/annular fillings at distance $\geq 4$ given in [GW1] and [GW3], that in this case the manifold $M$ is either $M_{1}, M_{2}$ or $M_{3}$, or is obtained from one of those by Dehn filling along one of the boundary components.

In Section 22 we show how the manifolds $M_{i}, 6 \leq i \leq 14$ may be realized as double branched covers. Using this, in Section 23 we show that the manifolds $M_{i}$ are hyperbolic. Finally, in Section 24 we give the applications to toroidal surgeries on knots in $S^{3}$.

\section{Preliminary Lemmas}

Throughout this paper, we will fix a hyperbolic 3-manifold $M$, with a torus $T_{0}$ as a boundary component. A compact surface properly embedded in $M$ is essential if it is $\pi_{1}$-injective, and is not boundary parallel. We use $a, b$ to denote the numbers 1 or 2 , with the convention that if they both appear in a statement then $\{a, b\}=\{1,2\}$.

A slope on $T_{0}$ is a toroidal slope if $M\left(r_{a}\right)$ is toroidal. Let $r_{a}$ be a toroidal slope on $T_{0}$. Denote by $\Delta=\Delta\left(r_{1}, r_{2}\right)$ the minimal geometric intersection number between $r_{1}$ and $r_{2}$. When $\Delta>5$ the manifolds $M$ have been determined in [Go]. We will always assume that $\Delta=4$ or 5 . Let $\hat{F}_{a}$ be an essential torus in $M\left(r_{a}\right)$, and let $F_{a}=\hat{F}_{a} \cap M$. If $M\left(r_{a}\right)$ is reducible then by [Wu1] and [Oh] we would have $\Delta \leq 3$, which is a contradiction. Therefore both $M\left(r_{a}\right)$ are irreducible.

Let $n_{a}$ be the number of boundary components of $F_{a}$ on $T_{0}$. Choose $\hat{F}_{a}$ in $M\left(r_{a}\right)$ so that $n_{a}$ is minimal among all essential tori in $M\left(r_{a}\right)$. Minimizing the number of components of $F_{1} \cap F_{2}$ by an isotopy, we may assume that $F_{1} \cap F_{2}$ consists of arcs and circles which are essential on both $F_{a}$. Denote by $J_{a}$ the attached solid torus in $M\left(r_{a}\right)$, and by $u_{i}\left(i=1, \ldots, n_{a}\right)$ the components of $\hat{F}_{a} \cap J_{a}$, which are all disks, labeled successively when traveling along $J_{a}$. Similarly let $v_{j}$ be the disk components of $\hat{F}_{b} \cap J_{b}$. Let $\Gamma_{a}$ be the graph on $\hat{F}_{a}$ with the $u_{i}$ 's as (fat) vertices, and the arc components of $F_{1} \cap F_{2}$ as edges. Similarly for $\Gamma_{b}$. The minimality of the number of components in $F_{1} \cap F_{2}$ and the minimality of $n_{a}$ imply that $\Gamma_{a}$ has no trivial loops, and that each disk face of $\Gamma_{a}$ in $\hat{F}_{a}$ has interior disjoint from $F_{b}$.

If $e$ is an edge of $\Gamma_{a}$ with an endpoint $x$ on a fat vertex $u_{i}$, then $x$ is labeled $j$ if $x$ is in $u_{i} \cap v_{j}$. In this case $e$ is called a $j$-edge in $\Gamma_{a}$, and an $i$-edge in $\Gamma_{b}$. Labels in $\Gamma_{a}$ are considered as mod $n_{b}$ integers; in particular, $n_{b}+1=1$. When going around $\partial u_{i}$, the labels of the endpoints of edges appear as $1,2, \ldots, n_{b}$ repeated $\Delta$ times. Label the endpoints of edges in $\Gamma_{b}$ similarly.

Each vertex of $\Gamma_{a}$ is given a sign according to whether $J_{a}$ passes $\hat{F}_{a}$ from the positive side or negative side at this vertex. Two vertices of $\Gamma_{a}$ are parallel if they have the same sign, otherwise they are antiparallel. Note that if $\hat{F}_{a}$ is a separating surface, then $n_{a}$ is even, and $v_{i}, v_{j}$ are parallel if and only if $i, j$ have 
the same parity. We use $\operatorname{val}(v, G)$ to denote the valence of a vertex $v$ in a graph $G$. If $G$ is clear from the context, we simply denote it by $\operatorname{val}(v)$.

When considering each family of parallel edges of $\Gamma_{a}$ as a single edge $\hat{e}$, we get the reduced graph $\hat{\Gamma}_{a}$ on $\hat{F}_{a}$. It has the same vertices as $\Gamma_{a}$. Each edge of $\hat{\Gamma}_{a}$ represents a family of parallel edges in $\Gamma_{a}$. We shall often refer to a family of parallel edges as simply a family.

Definition 2.1 (1) An edge of $\Gamma_{a}$ is a positive edge if it connects parallel vertices. Otherwise it is a negative edge.

(2) The graph $\Gamma_{a}$ is positive if all its vertices are parallel, otherwise it is non-positive. $p$

We use $\Gamma_{a}^{+}$(resp. $\Gamma_{a}^{-}$) to denote the subgraph of $\Gamma_{a}$ whose vertices are the vertices of $\Gamma_{a}$ and whose edges are the positive (resp. negative) edges of $\Gamma_{a}$. Similarly for $\hat{\Gamma}_{a}^{+}$and $\hat{\Gamma}_{a}^{-}$.

A cycle in $\Gamma_{a}$ consisting of positive edges is a Scharlemann cycle if it bounds a disk with interior disjoint from the graph, and all the edges in the cycle have the same pair of labels $\{i, i+1\}$ at their two endpoints, called the label pair of the Scharlemann cycle. A Scharlemann cycle containing only two edges is called a Scharlemann bigon. A Scharlemann cycle with label pair, say, $\{1,2\}$ will also be called a (12)-Scharlemann cycle. If $\Gamma_{b}$ contains a Scharlemann cycle with label pair $\{i, i \pm 1\}$, we shall sometimes abuse terminology and say that the vertex $u_{i}$ of $\Gamma_{a}$ is a label of a Scharlemann cycle. An extended Scharlemann cycle is a cycle of edges $\left\{e_{1}, \ldots, e_{k}\right\}$ such that there is a Scharlemann cycle $\left\{e_{1}^{\prime}, \ldots, e_{k}^{\prime}\right\}$ with $e_{i}$ parallel and adjacent to $e_{i}^{\prime}$ and $e_{i} \neq e_{j}^{\prime}, 1 \leq i, j \leq k$. If $\left\{e_{1}, \ldots, e_{k}\right\}$ is a Scharlemann cycle in $\Gamma_{a}$ then the subgraph of $\Gamma_{b}$ consisting of these edges and their vertices is called a Scharlemann cocycle.

A subgraph $G$ of a graph $\Gamma$ on a surface $F$ is essential if it is not contained in a disk in $F$. The following lemma contains some common properties of the graphs $\Gamma_{a}$. It can be found in [GW1, Lemma 2.2].

Lemma 2.2 (1) (The Parity Rule) An edge $e$ is a positive edge in $\Gamma_{1}$ if and only if it is a negative edge in $\Gamma_{2}$.

(2) A pair of edges cannot be parallel on both $\Gamma_{1}$ and $\Gamma_{2}$.

(3) If $\Gamma_{a}$ has a set of $n_{b}$ parallel negative edges, then on $\Gamma_{b}$ they form mutually disjoint essential cycles of equal length.

(4) If $\Gamma_{a}$ has a Scharlemann cycle, then $\hat{F}_{b}$ is separating. In particular, $\Gamma_{b}$ has the same number of positive and negative vertices, so $n_{b}$ is even, and two vertices $v_{i}, v_{j}$ of $\Gamma_{b}$ are parallel if and only if $i, j$ have the same parity.

(5) If $\Gamma_{a}$ has a Scharlemann cycle $\left\{e_{1}, \ldots, e_{k}\right\}$, then the corresponding Scharlemann cocycle on $\Gamma_{b}$ is essential.

(6) If $n_{b}>2$, then $\Gamma_{a}$ contains no extended Scharlemann cycle.

Let $\hat{e}$ be a collection of parallel negative edges on $\Gamma_{b}$, oriented from $v_{1}$ to $v_{2}$. Then $\hat{e}$ defines a permutation $\varphi:\left\{1, \ldots, n_{a}\right\} \rightarrow\left\{1, \ldots, n_{a}\right\}$, such that an edge $e$ in $\hat{e}$ has label $k$ at $v_{1}$ if and only if it has label $\varphi(k)$ at $v_{2}$. Call $\varphi$ the transition function associated to $\hat{e}$. Define the transition number to be the mod 
$n_{a}$ integer $s=s(\hat{e})$ such that $\varphi(k)=k+s$. If we reverse the orientation of $\hat{e}$ then the transition function is $\varphi^{-1}$, and the transition number is $-s$; hence if $\hat{e}$ is unoriented then $\varphi$ is well defined up to inversion, and $s(\hat{e})$ is well defined up to sign.

Lemma 2.3 (1) If a family of parallel negative edges in $\Gamma_{a}$ contains more than $n_{b}$ edges (in particular, if the family contains 3 edge endpoints with the same label), then $\Gamma_{b}$ is positive, and the transition function associated to this family is transitive.

(2) If $\Gamma_{a}$ contains two Scharlemann cycles with disjoint label pairs $\{i, i+1\}$ and $\{j, j+1\}$, then $i \equiv j \bmod 2$.

(3) If $n_{b}>2$ then a family of parallel positive edges in $\Gamma_{a}$ contains at most $n_{b} / 2+2$ edges, and if it does contain $n_{b} / 2+2$ edges, then $n_{b} \equiv 0 \bmod 4$.

(4) $\Gamma_{a}$ has at most four labels of Scharlemann cycles, at most two for each sign.

(5) A loop edge e and a non-loop edge $e^{\prime}$ on $\Gamma_{a}$ cannot be parallel on $\Gamma_{b}$.

(6) If $n_{b} \geq 4$ then $\Gamma_{a}$ contains at most $2 n_{b}$ parallel negative edges.

Proof. (1) This is obvious if $n_{b} \leq 2$, and it can be found in [GW1, Lemma 2.3] if $n_{b}>2$.

(2) and (3) are basically Lemmas 1.7 and 1.4 of [Wu1]. If $\Gamma_{a}$ has $n_{b} / 2+2$ parallel positive edges then the two outermost pairs form two Scharlemann bigons. One can then check the labels of these Scharlemann bigons and use (2) to show that $n_{b} \equiv 0 \bmod 4$.

(4) If $\Gamma_{a}$ has more than four labels of Scharlemann cycles, then either one can find two Scharlemann cycles with disjoint label pairs $\{i, i+1\}$ and $\{j, j+1\}$ such that $i-j \equiv 1 \bmod 2$, which is a contradiction to (2), or one can find three Scharlemann cycles with mutually disjoint label pairs, in which case one can replace $\hat{F}_{a}$ by another essential torus to reduce $n_{a}$ and get a contradiction. See [Wu1, Lemma 1.10].

If $\Gamma_{a}$ has three positive labels of Scharlemann cycles $u_{i_{j}}$ then it has negative labels of Scharlemann cycles $u_{i_{j}+\epsilon_{j}}$ for some $\epsilon_{j}= \pm 1$, which cannot all be the same, hence $\Gamma_{a}$ has at least 5 labels of Scharlemann cycles, contradicting the above.

(5) Since $e$ is positive on $\Gamma_{a}$, it is negative in $\Gamma_{b}$. If $e$ has endpoints on $u_{i}$ in $\Gamma_{a}$ then on $\Gamma_{b}$ its two endpoints are both labeled $i$, hence the corresponding transition number is 0 , so any edge $e^{\prime}$ parallel to $e$ on $\Gamma_{b}$ must also have the same label at its two endpoints, which implies that $e^{\prime}$ is a loop on $\Gamma_{a}$.

(6) This is [Go, Corollary 5.5].

Lemma 2.4 If a label $i$ appears twice among the endpoints of a family $\hat{e}$ of parallel positive edges in $\Gamma_{a}$, then $i$ is a label of a Scharlemann bigon in $\hat{e}$. In particular, if $\hat{e}$ has more than $n_{b} / 2$ edges, then it contains a Scharlemann bigon.

Proof. Since the edges are positive, by the parity rule $i$ cannot appear at both endpoints of a single edge in this family. Let $e_{1}, e_{2}, \ldots, e_{k}$ be consecutive edges 
of $\hat{e}$ such that $e_{1}$ and $e_{k}$ have $i$ as a label. Now $k$ must be even, otherwise the edge $e_{(k+1) / 2}$ would have the same label at its two endpoints. If $k \geq 4$ and $n_{b}>2$ one can see that these edges contain an extended Scharlemann cycle, which contradicts Lemma 2.2(6). Therefore $k=2$ or $n_{b}=2$, in which case $e_{1}, e_{2}$ form a Scharlemann bigon with $i$ as a label.

If $\hat{e}$ has more than $n_{b} / 2$ edges, then it has more than $n_{b}$ endpoints, so some label must appear twice.

Lemma 2.5 $\hat{\Gamma}_{a}$ contains at most $3 n_{a}$ edges.

Proof. Let $V, E, F$ be the number of vertices, edges and disk faces of $\hat{\Gamma}_{a}$. Then $V-E+F \geq 0$ (the inequality may be strict if there are some non-disk faces.) Each face of $\hat{\Gamma}_{a}$ has at least three edges, hence we have $3 F \leq 2 E$. Solving those two inequalities gives $E \leq 3 \mathrm{~V}$.

Lemma 2.6 If $n_{b}>4$ then the vertices of $\Gamma_{a}$ cannot all be parallel.

Proof. By Lemma 2.5 the reduced graph $\hat{\Gamma}_{a}$ has at most $3 n_{a}$ edges. For any $i$, since $v_{i}$ on $\Gamma_{b}$ has valence at least $4 n_{a}$, there are $4 n_{a} i$-edges on $\Gamma_{a}$, hence two of them must be parallel, so $i$ is a label of a Scharlemann cycle. Since there are at most 4 such labels (Lemma $2.3(4)$ ), we would have $n_{b} \leq 4$, contradicting the assumption.

A vertex $v$ of a graph is a full vertex if all edges incident to it are positive.

Lemma 2.7 Suppose $n_{b}>4$. Then

(1) a family of parallel negative edges in $\Gamma_{b}$ contains at most $n_{a}$ edges, hence any label $i$ appears at most twice among the endpoints of such a family;

(2) two families of positive edges in $\Gamma_{a}$ adjacent at a vertex contain at most $n_{b}+2$ edges; and

(3) three families of positive edges in $\Gamma_{a}$ adjacent at a vertex contain at most $2 n_{b}$ edges, and if there are $2 n_{b}$ then $n_{b}=6$.

Proof. (1) If a family of parallel negative edges on $\Gamma_{b}$ contains more than $n_{a}$ edges then by Lemma 2.3(1) all vertices of $\Gamma_{a}$ are parallel, which contradicts Lemma 2.6.

If $i$ appears three times among the endpoints of a family of parallel negative edges in $\Gamma_{b}$ then this family would contain more than $n_{a}$ edges, which is a contradiction.

(2) By Lemma 2.3(3) a family of parallel positive edges contains $r \leq n_{b} / 2+2$ edges. If two adjacent families $\hat{e}_{1}, \hat{e}_{2}$ contain more than $n_{b}+2$ edges, then one of them, say $\hat{e}_{1}$, has $n_{b} / 2+2$ edges while the other one has either $n_{b} / 2+1$ or $n_{b} / 2+2$ edges. Now $\hat{e}_{1}$ contains two Scharlemann bigons, which must appear on the two sides of the family because there is no extended Scharlemann cycle. There is also at least one Scharlemann bigon in $\hat{e}_{2}$. Examining the labels of these Scharlemann bigons we can see that they contain at least 5 labels, which contradicts Lemma 2.3(4). 
(3) Assume the three families contain $r \geq 2 n_{b}$ edges. Then one of the families contains more than $n_{b} / 2$ edges, so by Lemma 2.2(4) $n_{b}$ is even. By (2) two adjacent families of parallel edges contain at most $n_{b}+2$ edges, while by Lemma $2.3(3)$ the other family has at most $n_{b} / 2+2$ edges, so we have $2 n_{b} \leq r \leq\left(n_{b}+2\right)+\left(n_{b} / 2+2\right)$, which gives $n_{b} \leq 8$.

If $n_{b}=8$ then the above inequalities force the three families to have $6,4,6$ edges, and we see that all 8 labels appear as labels of Scharlemann bigons, which contradicts Lemma 2.3(4). So we must have $n_{b}=6$. By Lemma 2.3(3) we have $2 n_{b} \leq r \leq 3\left(n_{b} / 2+1\right)=12=2 n_{b}$. Hence $r=2 n_{b}$.

Lemma 2.8 If a vertex $u_{i}$ of $\Gamma_{a}$ is incident to more than $n_{b}$ negative edges, then $\Gamma_{b}$ has a Scharlemann cycle.

Proof. In this case there are $n_{b}+1$ positive $i$-edges in $\Gamma_{b}$, which cut the surface $F_{b}$ into faces, at least one of which is a disk face in the sense that it is a topological disk whose interior contains no vertices of $\Gamma_{b}$. Hence the subgraph of $\Gamma_{b}$ consisting of these edges is a $x$-edge cycle in the sense of Hayashi-Motegi [HM, Page 4468]. By [HM, Proposition 5.1] a disk face of this $x$-edge cycle contains a disk face of a Scharlemann cycle.

Consider a graph $G$ on a closed surface $F$, and assume that $G$ has no isolated vertex. If the vertices of $G$ have been assigned \pm signs (for example $\hat{\Gamma}_{a}^{+}$), let $X$ be the union of $G$ and all its faces $\sigma$ such that all vertices on $\partial \sigma$ have the same sign, otherwise let $X$ be the union of $G$ and all its disk faces. A vertex $v$ of $G$ is an interior vertex if it lies in the interior of $X$. A vertex $v$ of $G$ is a cut vertex if a regular neighborhood of $v$ in $X$ with $v$ removed is not connected. A vertex $v$ of $G$ is a boundary vertex if it is not an interior or cut vertex. Note that if $G=\hat{\Gamma}_{a}^{+}$then an interior vertex is a full vertex. Alternatively, let $\delta(v)$ be the number of corners around $v$ which lie in $X$. Then $v$ is an interior vertex if $\delta(v)=\operatorname{val}(v, G)$, a boundary vertex if $\delta(v)=\operatorname{val}(v, G)-1$, and a cut vertex if $\delta(v) \leq \operatorname{val}(v, G)-2$.

Given a graph $G$ on a surface $D$, let $c_{i}(G)$ be the number of boundary vertices of $G$ with valence $i$. Define

$$
\begin{aligned}
& \varphi(G)=6 c_{0}(G)+3 c_{1}(G)+2 c_{2}(G)+c_{3}(G) \\
& \psi(G)=c_{0}(G)+c_{1}(G)+c_{2}(G)+c_{3}(G) .
\end{aligned}
$$

Note that $\psi(G)$ is the number of boundary vertices of $G$ with valence at most 3 .

Lemma 2.9 Let $G$ be a connected reduced graph in a disk $D$ such that any interior vertex of $G$ has valence at least 6 . Then $\varphi(G) \geq 6$. Moreover, if $G$ is not homeomorphic to an arc or a single point then $\psi(G) \geq 3$.

Proof. Let $X$ be the union of $G$ and all its disk faces. The result is obviously true if $G$ is a tree. So we assume that $G$ has some disk faces. 
First assume that $X$ has no cut vertex, so it is a disk, and $c_{0}(G)=c_{1}(G)=0$. The double of $G$ along $\partial X$ is then a graph $\tilde{G}$ on the double of $X$, which is a sphere. Note that the valence of a vertex $v$ of $\tilde{G}$ is either at least 6 , or it is 2 or 4 when $v$ is a boundary vertex of $G$ with valence 2 or 3 , respectively. Since each face has at least three edges, an Euler characteristic argument gives

$$
2=V-E+F \leq V-\frac{1}{3} E=\sum_{i}\left(1-\frac{1}{6} \operatorname{val}\left(v_{i}, \tilde{G}\right)\right) \leq \frac{2}{3} c_{2}(G)+\frac{1}{3} c_{3}(G)
$$

Therefore $\varphi(G)=2 c_{2}(G)+c_{3}(G) \geq 6$. Since $c_{0}(G)=c_{1}(G)=0$, we also have $\psi(G)=c_{2}(G)+c_{3}(G) \geq \frac{1}{2} \varphi(G) \geq 3$.

Now assume that $X$ has a cut vertex $v$. Since $G$ is connected and contained in a disk, $X$ is simply connected, so we can write $X=X_{1} \cup X_{2}$, where $X_{i}$ are subcomplexes of $X$ such that $X_{1} \cap X_{2}=v$, and $G_{i}=G \cap X_{i}$ are nontrivial connected subgraphs of $G$. The valence of $v$ in $G_{i}$ is at least 1 , so its contribution to $\varphi\left(G_{i}\right)$ is at most 3 . Hence by induction we have

$$
\varphi(G) \geq\left(\varphi\left(G_{1}\right)-3\right)+\left(\varphi\left(G_{2}\right)-3\right) \geq 6 .
$$

By assumption $X$ is not homeomorphic to an arc, so at least one of the $X_{i}$, say $X_{1}$, is not homeomorphic to an arc, and the other one has at least 2 boundary vertices of valence at most 3 , whether it is homeomorphic to an arc or not. Hence

$$
\psi(G) \geq \psi\left(G_{1}\right)+\psi\left(G_{2}\right)-2 \geq 3+2-2=3
$$

Lemma 2.10 Let $G$ be a reduced graph on a torus $T$ with no interior or isolated vertex. Let $V$ and $E$ be the number of vertices and edges of $G$, and let $k$ be the number of boundary vertices of $G$.

(1) $k \geq E-V$, and equality holds if and only if all disk face of $G$ are triangles, all non-disk faces are annuli, and each cut vertex has exactly two corners on annular faces.

(2) $G$ has at most $2 V$ edges.

Proof. (1) Let $D$ be the number of disk faces of $G$. Then $0=\chi(T) \leq V-E+D$, and equality holds if and only if all non-disk faces are annuli. Thus $D \geq E-V$. For each vertex $u$ of $G$, let $\delta(u)$ be the number of corners of disk faces incident to $u$. Then $\sum_{u} \operatorname{val}(u)=2 E$, and $\sum_{u} \delta(u) \geq 3 D \geq 3(E-V)$. Since there is no isolated or interior vertex, we have $\operatorname{val}(u)-\delta(u) \geq 1$, and equality holds if and only if $u$ is a boundary vertex. Let $p$ be the number of non-boundary vertices. Then

$$
p \leq \sum_{u}(\operatorname{val}(u)-\delta(u)-1) \leq 2 E-3(E-V)-V=2 V-E .
$$

It follows that the number of boundary vertices is $k=V-p \geq E-V$, and equality holds if and only if (i) $V-E+D=0$, i.e. non-disk faces are annuli, (ii) 
$\sum_{u} \delta(u)=3 D$, so all disk faces are triangles, and (iii) $\operatorname{val}(u)-\delta(u)-1=1$ for any cut vertex, i.e. each cut vertex has exactly two corners not on disk faces.

(2) Since the number of boundary vertices is at most $V$, by (1) we have $V \geq k \geq E-V$, hence $E \leq 2 V$.

Lemma 2.11 Suppose all interior vertices of $\hat{\Gamma}_{a}^{+}$have valence at least 6 , and all boundary vertices of $\hat{\Gamma}_{a}^{+}$have valence at least 4 . Let $G$ be a component of $\hat{\Gamma}_{a}^{+}$. Then either (i) $G$ is topologically an essential circle on the torus $\hat{F}_{a}$, or (ii) $G$ has no cut vertex, all interior vertices of $G$ are of valence exactly 6 , and all boundary vertices of $G$ are of valence exactly 4 .

Proof. Let $X$ be the union of $G$ and all its disk faces. If $X$ is the whole torus then all vertices are interior vertices, and an easy Euler characteristic argument shows that all vertices must be of valence 6 , so (ii) follows. Also, by Lemma $2.9 X$ is not in a disk in $\hat{F}_{a}$ as otherwise $G$ would have a boundary vertex of valence at most 3 . Therefore we may assume that $X$ has the homotopy type of a circle.

First assume that $X$ has a cut vertex $v$. Recall that $X$ is homotopy equivalent to a circle, so if $X-v$ is not connected, then $v$ cuts off a subcomplex $W$ of $X$ which lies in a disk in $\hat{F}_{a}$. By Lemma 2.9 the graph $G \cap W$ has at least two boundary vertices of valence at most 3 , hence at least one such vertex $v^{\prime}$ other than $v$, which contradicts the assumption because $v^{\prime}$ is then a boundary vertex of $\hat{\Gamma}_{a}^{+}$of valence at most 3 . Therefore we may assume that $X-v$ is connected. Since $X$ has the homotopy type of a circle, $X$ cut at $v$ is a simply connected planar complex $W$, and $X$ is obtained by identifying exactly two points of $W$. Let $G^{\prime}$ be the corresponding graph on $W$. We may assume that $X$ is not a circle as otherwise (i) is true. Thus $W$ is not homeomorphic to an arc. Therefore by Lemma 2.9 we have $\psi\left(G^{\prime}\right) \geq 3$, hence $G^{\prime}$ has at least one boundary vertex $v^{\prime}$ of valence at most 3 which is not identified to $v$ in $G$. By definition $v^{\prime}$ is a boundary vertex of $\hat{\Gamma}_{a}^{+}$of valence at most 3 , which is a contradiction. This completes the proof that $X$ has no cut vertex.

We may now assume that $X$ is an annulus, so all vertices of $G$ are either interior vertices of valence at least 6 in the interior of $X$, or boundary vertices of valence at least 4 on $\partial X$. Consider the double $G^{\prime \prime}$ of $G$ on the double of $X$ along $\partial X$. Since each boundary vertex of $G$ of valence $k$ gives rise to a vertex of valence $2 k-2$ in $G^{\prime \prime}$, we see that $G^{\prime \prime}$ is a reduced graph on a torus such that all of its vertices have valence at least 6 . An Euler characteristic argument shows that all vertices of $G^{\prime \prime}$ must have valence exactly 6 , hence (ii) follows.

Lemma 2.12 If $M\left(r_{a}\right)$ contains a Klein bottle $K$, then

(1) $T=\partial N(K)$ is an essential torus in $M\left(r_{a}\right)$; and

(2) $K$ intersects the core $K_{a}$ of the Dehn filling solid torus at no less than $n_{a} / 2$ points.

Proof. $T$ bounds a twisted $I$-bundle over the Klein bottle $N(K)$ on one side. Since $M\left(r_{a}\right)$ is assumed irreducible, if $T$ is compressible on the other side then 
$M\left(r_{a}\right)$ is a Seifert fiber space over a sphere with (at most) three singular fibers of indices $(2,2, p)$ for some $p$, and if $T$ is boundary parallel then $M\left(r_{a}\right)$ is a twisted $I$-bundle over the Klein bottle. Either case contradicts the assumption that $M\left(r_{a}\right)$ is toroidal. Therefore $T$ is an essential torus. If $\left|K \cap K_{a}\right|<n_{a} / 2$ then $T$ would intersect $K_{a}$ in less than $n_{a}$ points, contradicting the choice of $n_{a}$.

Lemma 2.13 Suppose $n_{a}>2$, and $\Gamma_{b}$ has both a 12-Scharlemann bigon $e_{1} \cup e_{2}$ and a 23-Scharlemann bigons $e_{3} \cup e_{4}$. If $e_{1} \cup e_{2}$ and $e_{3} \cup e_{4}$ are isotopic on $\hat{F}_{a}$, then the disk face $D$ they bound on $\hat{F}_{a}$ contains at least $\left(n_{a} / 2\right)-1$ vertices in its interior.

Proof. Let $m$ be the number of vertices in the interior of $D$. Let $D_{1}, D_{2}$ be the disk faces of (12)- and (23)-Scharlemann bigons in $\Gamma_{b}$. Shrinking the Dehn filling solid torus of $M\left(r_{a}\right)$ to its core $K_{a}$, the union $D_{1} \cup D_{2} \cup D$ is a Klein bottle $Q$ in $M\left(r_{a}\right)$. A regular neighborhood of $Q$ intersects $K_{a}$ at an arc from $u_{1}$ to $u_{2}$ then to $u_{3}$, and one arc for each vertex of $\Gamma_{a}$ in the interior of $D$. Hence $Q$ can be perturbed to intersect $K_{a}$ at $1+m$ points. By Lemma 2.12(2) we have $m+1 \geq n_{a} / 2$, hence the result follows.

An edge $e$ of $\Gamma_{a}$ is a co-loop edge if it has the same label on its two endpoints, in other words, it is a loop on the other graph $\Gamma_{b}$. Given a codimension 1 manifold $X$ in a manifold $Y$, use $Y \mid X$ to denote the manifold obtained by cutting $Y$ along $X$.

Lemma 2.14 Let $\hat{e}$ be a family of negative edges in $\Gamma_{a}$. Let $G$ be the subgraph of $\Gamma_{b}$ consisting of the edges of $\hat{e}$ and their vertices.

(1) Each cycle component of $G$ is an essential loop on $\hat{F}_{b}$.

(2) (The 3-Cycle Lemma.) G cannot contain three disjoint cycles; in particular, $\Gamma_{a}$ cannot have three parallel co-loop edges.

(3) (The 2-Cycle Lemma.) If $\Gamma_{b}$ is positive then $G$ cannot contain two disjoint cycles; in particular, $\Gamma_{a}$ cannot have two parallel co-loop edges.

Proof. (1) Assume to the contrary that some cycle component of $G$ is inessential on $\hat{F}_{b}$. Let $D$ be a disk bounded by an innermost cycle component of $G$, and let $D^{\prime}$ be the bigon disks on $F_{a}$ between edges of $\hat{e}$. Let $V_{b}$ be the Dehn filling solid torus in $M\left(r_{b}\right)$. Then a regular neighborhood $W$ of $D \cup V_{b} \cup D^{\prime}$ is a solid torus containing the core of $V_{b}$ as a cable knot winding along the longitude at least twice. See the proof of [GLi, Proposition 1.3]. In this case $W \cap M$ is a cable space, which is a contradiction to the assumption that $M$ is a hyperbolic manifold.

(2) Let $\hat{e}=e_{1} \cup \ldots \cup e_{k}$, oriented consistently, with tails at $u^{\prime}$ and heads at $u^{\prime \prime}$ on $\Gamma_{a}$. Let $s$ be the transition number of $\hat{e}$. We may assume that $e_{i}$ has label $i$ at its tail, so it has label $i+s$ at its head. Let $D_{j}$ be the bigon on $\hat{F}_{a}$ between $e_{j}$ and $e_{j+1}$.

If $k>n_{b}$ then by Lemma 2.3(1) the transition function associated with $\hat{e}$ has only one orbit, hence we may assume $k \leq n_{b}$. On $\Gamma_{b}$ these edges form disjoint 
cycles and chains. Assume there are at least three cycles. Then $e_{1}, e_{2}, e_{3}$ belong to three distinct cycles $C_{1}, C_{2}, C_{3}$. Thus for $i=1,2,3$,

$$
C_{i}=e_{i} \cup e_{i+s} \cup \ldots \cup e_{i+(p-1) s}
$$

is an oriented cycle on $\hat{F}_{b}$ for some fixed $p$. By (1) these are essential loops on $\hat{F}_{b}$, so they are parallel as unoriented loops.

Each bigon $D_{1+j s}$ gives a parallelism between an edge of $C_{1}$ and an edge of $C_{2}$, hence when shrinking the Dehn filling solid torus $V_{b}$ to its core knot $K_{b}$, the union $A_{1}=\cup D_{1+j s}$ is an annulus in $M\left(r_{b}\right)$ with $\partial A_{1}=C_{1} \cup C_{2}$. Similarly, $A_{2}=\cup D_{2+j s}$ is an annulus in $M\left(r_{b}\right)$ with $\partial A_{2}=C_{2} \cup C_{3}$. These $A_{i}$ are essential in $M\left(r_{b}\right) \mid \hat{F}_{b}$, the manifold obtained from $M\left(r_{b}\right)$ by cutting along $\hat{F}_{b}$, otherwise $K_{b}$ would be isotopic to a curve having fewer intersections with $\hat{F}_{b}$.

Let $A_{1}^{\prime}, A_{2}^{\prime}, A_{3}^{\prime}$ be the annuli $\hat{F}_{b} \mid\left(C_{1} \cup C_{2} \cup C_{3}\right)$, with $\partial A_{i}^{\prime}=C_{i} \cup C_{i+1}$ (subscripts mod 3.) Let $m_{i}$ be the number of times that $K_{b}$ intersects the interior of $A_{i}^{\prime}$. Then

$$
\sum m_{i}+3 p=n_{b}
$$

The annulus $A_{i}$ is said to be of type I if a regular neighborhood of $\partial A_{i}$ lies on the same side of $\hat{F}_{b}$, otherwise it is of type II. Note that if $\hat{F}_{b}$ is separating then $A_{i}$ must be of type I. There are several possibilities. In each case one can find an essential torus $T^{\prime}$ in $M\left(r_{b}\right)$ which has fewer intersections with $K_{b}$. This will contradict the choice of $\hat{F}_{b}$ and complete the proof of (1).

Case 1. $C_{2}$ is anti-parallel to both $C_{1}$ and $C_{3}$.

In this case each $T_{i}=A_{i} \cup A_{i}^{\prime}$ is a Klein bottle for $i=1,2$, which can be perturbed to intersect $K_{b}$ at $p+m_{i}$ points. Since $\sum m_{i}+3 p=n_{b}$, either $T_{1}$ or $T_{2}$ can be perturbed to intersect $K_{b}$ at fewer than $n_{b} / 2$ points, contradicting Lemma 2.12.

Case 2. $C_{2}$ is anti-parallel to $C_{1}$, say, and parallel to the other cycle $C_{3}$.

Let $T_{1}=A_{1} \cup A_{1}^{\prime}$ and $T_{2}=A_{1} \cup A_{2} \cup A_{3}^{\prime}$. Then $T_{i}$ are Klein bottles, and they can be perturbed to intersect $K_{b}$ at $p+m_{1}$ and $m_{3}$ points, respectively. One of these contradicts Lemma 2.12.

Case 3. $C_{2}$ is parallel to both $C_{1}$ and $C_{3}$.

If one of the $A_{i}$, say $A_{1}$, is of type II, then $T_{1}=A_{1} \cup A_{1}^{\prime}$ is a non-separating torus (because it can be perturbed to intersect $\hat{F}_{b}$ transversely at a single circle), and it intersects $K_{b}$ at $p+m_{i}<n_{b}$ points. Since $M\left(r_{b}\right)$ is irreducible, $T_{1}$ is incompressible and hence essential, which contradicts the choice of $\hat{F}_{b}$.

If both $A_{i}$ are of type I then one can show that $A_{1} \cup A_{2} \cup A_{3}^{\prime}$ is an essential torus $T$ which can be perturbed to intersect $K_{b}$ in $m_{3}+p<n_{b}$ points. The proof is standard: The torus $\hat{F}_{b}$ and the annuli $A_{1}, A_{2}$ cut $M\left(r_{b}\right)$ into a manifold whose boundary contains four tori $T_{1}=A_{1} \cup A_{1}^{\prime}, T_{2}=A_{1} \cup A_{2}^{\prime} \cup A_{3}^{\prime}, T_{3}=A_{2} \cup A_{2}^{\prime}$, and $T_{4}=A_{2} \cup A_{1}^{\prime} \cup A_{3}^{\prime}$. Each of these tori $T_{i}$ can be perturbed to have fewer than $n_{b}$ intersections with the knot $K_{b}$, and hence bounds a manifold $W_{i}$ which is either a solid torus or a $T^{2} \times I$ between $T_{i}$ and a component of $\left.\partial M_{(} r_{b}\right)$. Moreover, if $W_{i}$ is a solid torus $W_{i}$ then the annulus $T_{i} \cap \hat{F}_{b}$ is essential on $\partial W_{i}$ 
in the sense that it is neither meridional nor longitudinal (otherwise $\hat{F}_{b}$ would be compressible or could be isotoped to have fewer intersections with $K_{b}$ ). Now we have $M\left(r_{b}\right)=\left(W_{1} \cup W_{4}\right) \cup\left(W_{2} \cup W_{3}\right)=W^{\prime} \cup W^{\prime \prime}$, with $W^{\prime} \cap W^{\prime \prime}$ a torus $T=A_{1} \cup A_{2} \cup A_{3}^{\prime}$ which can be perturbed to intersect $K_{b}$ at $m_{3}+p<n_{b}$ points. Since $W^{\prime}=W_{1} \cup_{A_{1}^{\prime}} W_{4}$ and $A_{1}^{\prime}$ is essential in both $W_{1}$ and $W_{4}, T$ is incompressible and not boundary parallel in $W^{\prime}$; similarly for $W^{\prime \prime}$. It follows that $T$ is a contradiction to the choice of $\hat{F}_{b}$.

(3) The proof of this part is much simpler. Let $A_{1}, A_{1}^{\prime}$ be as above, and let $A_{1}^{\prime \prime}$ be the complement of $A_{1}^{\prime}$ on $\hat{F}_{b}$. If $C_{1}, C_{2}$ are parallel then $A_{1} \cup A_{1}^{\prime}$ is a nonseparating torus in $M\left(r_{b}\right)$ which can be perturbed to intersect $K_{b}$ less than $n_{b}$ times, contradicting the choice of $\hat{F}_{b}$. If $C_{1}, C_{2}$ are anti-parallel then $A_{1} \cup A_{1}^{\prime}$ and $A_{1} \cup A_{1}^{\prime \prime}$ are Klein bottles, which can be perturbed to intersect $K_{b}$ at a total of $n-2 p$ points, where $p$ is the number of vertices in $C_{i}$; hence one of those will intersect $K_{b}$ less than $n_{b} / 2$ times, which contradicts Lemma 2.12.

When studying Dehn surgery via intersection graphs, we usually fix the surfaces $F_{1}, F_{2}$, and hence the graphs $\Gamma_{1}, \Gamma_{2}$ are also fixed. The following technique will allow us to modify the surfaces and hence the graphs in certain situation. Lemma 2.15 will be used in the proofs of Lemmas 12.16 and 19.6.

Consider two surfaces $F_{1}, F_{2}$ in a 3 -manifold $M$ with boundary slopes $r_{1}, r_{2}$ respectively and suppose they intersect minimally. Let $\Gamma_{a}, \Gamma_{b}$ be the intersection graphs on $\hat{F}_{1}, \hat{F}_{2}$, respectively. Let $\alpha$ be a proper arc on a disk face $D$ of $\Gamma_{a}$ with boundary on edges of $\Gamma_{a}$. Then one can replace two small arcs of $\Gamma_{a}$ centered at $\partial \alpha$ by two parallel copies of $\alpha$ to obtain a new graph $\Gamma_{a}^{\prime}$, called the graph obtained from $\Gamma_{a}$ by surgery along $\alpha$.
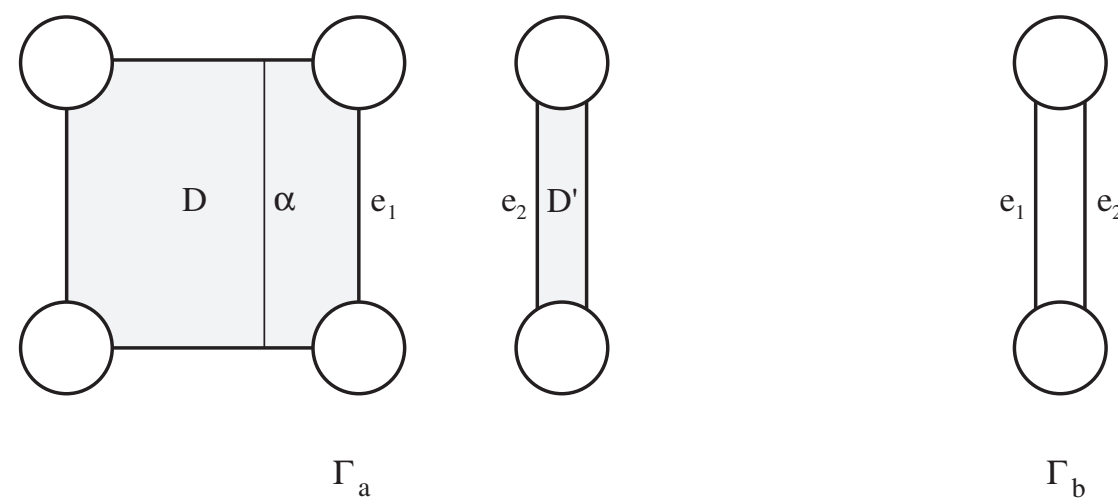

$\Gamma_{\mathrm{a}}$

$\Gamma_{\mathrm{b}}$

Figure 2.1

A face $D^{\prime}$ of $\Gamma_{a}$ is called a coupling face to another face $D$ of $\Gamma_{a}$ along an edge $e_{1}$ of $D$ if $D^{\prime}$ has an edge $e_{2}$ such that $e_{1}, e_{2}$ are adjacent parallel edges on $\Gamma_{b}$, and the neighborhoods in $D$ and $D^{\prime}$ of the $e_{i}$ 's lie (locally) on the same side of $\hat{F}_{b}$. Note that this is independent of whether $\hat{F}_{b}$ is orientable or separating in 
$M$. See Figure 2.1. By definition $D$ has no coupling face along $e_{1}$ if $e_{1}$ has no parallel edge on $\Gamma_{b}$, one coupling face along $e_{1}$ if $e_{1}$ has some parallel edges and is a border edge of the family, and two otherwise. A 4-gon face $D$ of $\Gamma_{a}$ looks like a "saddle surface" in $M \mid F_{b}$. In general it is not possible to push the saddle up or down to change the intersection graph. However, if some coupling face to an edge of $D$ is a bigon then this is possible. See Figure 2.2. More explicitly, we have the following lemma.

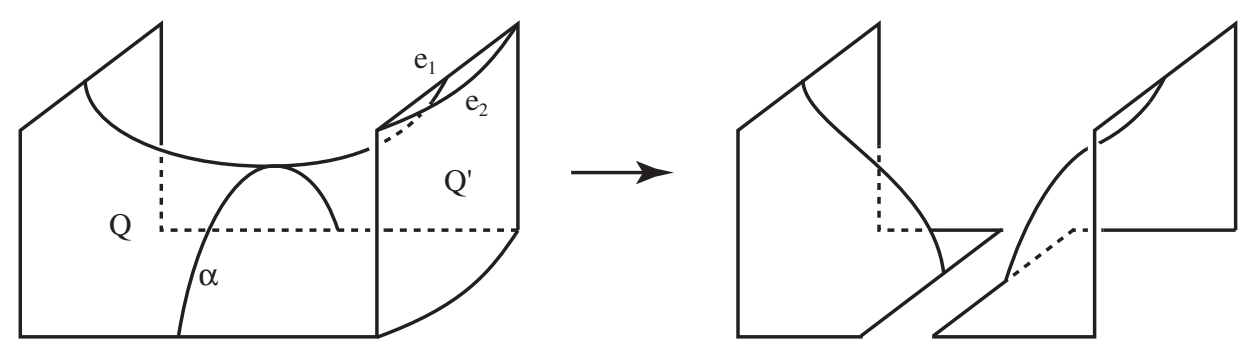

Figure 2.2

Lemma 2.15 Let $\Gamma_{1}, \Gamma_{2}$ be a pair of intersection graphs. Let $Q$ be a face of $\Gamma_{a}$, and let $e$ be an edge on $\partial Q$. Let $\alpha$ be an arc on $Q$ with boundary in the interior of edges of $\Gamma_{a}$, cutting off a disk $B_{1}$ containing e and exactly two corners of $Q$. If some coupling face $Q^{\prime}$ of $Q$ along $e$ is a bigon, then $F_{a}$ can be isotoped so that the new intersection graph $\Gamma_{a}^{\prime}$ is obtained from $\Gamma_{a}$ by surgery along $\alpha$.

Proof. Cut $M$ along $F_{b}$. Then the face $Q$ is as shown in Figure 2.2. Let $B_{2}$ be the bigon in $\Gamma_{b}$ between $e$ and the edge $e^{\prime}$ on $Q^{\prime}$. After shrinking the Dehn filling solid torus $V_{b}$ to its core knot $K_{b}$, the union $B_{1} \cup B_{2} \cup Q^{\prime}$ is a disk $Q^{\prime \prime}$ with boundary the union of $\alpha$ and an arc on $\hat{F}_{b}$. Pushing $Q^{\prime \prime}$ off $K_{b}$ gives a disk $P$ in $M$ which has boundary the union of $\alpha$ and an $\operatorname{arc}$ on $F_{b}$, and has interior disjoint from $F_{b} \cup F_{a}$. Therefore we can isotope $F_{a}$ through this disk $P$ to get a new surface $F_{a}^{\prime}$. It is clear that the new intersection graph $\Gamma_{a}^{\prime}$ is obtained from $\Gamma_{a}$ by surgery along $\alpha$.

Let $u$ be a vertex of $\Gamma_{a}$, and $P, Q$ two edge endpoints on $\partial u$. Let $I$ be the interval on $\partial u$ from $P$ to $Q$ along the direction induced by the orientation of $u$. The edge endpoints of $\Gamma_{a}$ cut $I$ into $k$ subintervals for some $k$. Then the distance from $P$ to $Q$ on $\partial u$ is defined as $d_{u}(P, Q)=k$. Some times we also use $d_{\Gamma_{a}}(P, Q)$ to denote $d_{u}(P, Q)$. If $P, Q$ are the only edge endpoints of $e_{1}, e_{2}$ on $\partial u$, respectively, then we define $d_{u}\left(e_{1}, e_{2}\right)=d_{u}(P, Q)$. Notice that if the valence of $u$ is $m$, then $d_{u}(Q, P)=m-d_{u}(P, Q)$. The following lemma can be found in [Go].

Lemma 2.16 [Go, Lemma 2.4] (i) Suppose $P, Q \in \partial u_{i} \cap \partial v_{k}$ and $R, S \in$ $\partial u_{j} \cap \partial v_{l}$. If $d_{u_{i}}(P, Q)=d_{u_{j}}(R, S)$ then $d_{v_{k}}(P, Q)=d_{v_{l}}(R, S)$.

(ii) Suppose that $P \in u_{i} \cap v_{k}, Q \in u_{i} \cap v_{l}, R \in u_{j} \cap v_{k}$, and $S \in u_{j} \cap v_{l}$. If $d_{u_{i}}(P, Q)=d_{u_{j}}(R, S)$, then $e_{v_{k}}(P, R)=d_{v_{l}}(Q, S)$. 
Suppose two edges $e_{1}, e_{2}$ of $\Gamma_{a}$ connect the same pair of vertices $u_{i}, u_{j}$. Let $p_{k}, q_{k}$ be the endpoints of $e_{k}$ on $u_{i}, u_{j}$, respectively, $k=1,2$. Then $e_{1}, e_{2}$ are equidistant if $d_{u_{i}}\left(p_{1}, p_{2}\right)=d_{u_{j}}\left(q_{2}, q_{1}\right)$. (Note that the orders of the edge endpoints have been reversed.) Thus for example a pair of parallel positive edges is always equidistant, but a pair of parallel negative edges is not unless their distance is exactly half of the valence of the vertices.

Note that when $u_{i} \neq u_{j}$ the above equation can be written as $d_{u_{i}}\left(e_{1}, e_{2}\right)=$ $d_{u_{j}}\left(e_{2}, e_{1}\right)$. When $u_{i}=u_{j}, d_{u_{i}}\left(e_{1}, e_{2}\right)$ is not defined, and there are two choices for the pair $p_{k}, q_{k}$, but one can check that whether the equality $d_{u_{i}}\left(p_{1}, p_{2}\right)=$ $d_{u_{j}}\left(q_{2}, q_{1}\right)$ holds is independent of the choice of $p_{i}, q_{i}$.

The following lemma is called the Equidistance Lemma. It follows from Lemma 2.16, and can also be found in [GW1].

Lemma 2.17 [GW1, Lemma 2.8] Let $e_{1}, e_{2}$ be a pair of edges with $\partial e_{1}=\partial e_{2}$ in both $\Gamma_{1}$ and $\Gamma_{2}$. Then $e_{1}, e_{2}$ are equidistant in $\Gamma_{1}$ if and only if they are equidistant in $\Gamma_{2}$.

Given two oriented slopes $r_{1}, r_{2}$ on $T_{0}$, choose an oriented meridian-longitude pair $m, l$ on the torus $T_{0}$ so that $r_{1}=m$, then the slope $r_{2}$ is homologous to $J m+\Delta l$ for some mod $\Delta$ integer $J=J\left(r_{1}, r_{2}\right)$, called the jumping number between $r_{1}, r_{2}$. Note that if $\Delta=4$, then $J= \pm 1$, and if $\Delta=5$, then $J= \pm 1$ or \pm 2 . The following lemma is call the Jumping Lemma and can be found in [GW1].

Lemma 2.18 [GW1, Lemma 2.10] Let $P_{1}, \ldots, P_{\Delta}$ be the points of $\partial u_{i} \cap \partial v_{j}$, labeled successively on $\partial u_{i}$. Let $J=J\left(r_{1}, r_{2}\right)$ be the jumping number of $r_{1}, r_{2}$. Then on $v_{j}$ these points appear in the order of $P_{J}, P_{2 J}, \ldots, P_{\Delta J}$. In particular, they appear successively as $P_{1}, \ldots, P_{\Delta}$ along some direction of $\partial v_{j}$ if and only if $J= \pm 1$.

Lemma 2.19 Let $e_{1} \cup \ldots \cup e_{p}$ and $e_{1}^{\prime} \cup \ldots \cup e_{q}^{\prime}$ be two sets of parallel edges on $\Gamma_{a}$. Suppose $e_{1}$ is parallel to $e_{1}^{\prime}$ and $e_{p}$ parallel to $e_{q}^{\prime}$ on $\Gamma_{b}$. Then $p=q$.

Proof. Let $D_{1}, D_{2}, D_{3}, D_{4}$ be the disks realizing the parallelisms of $e_{1} \cup e_{p}$ and $e_{1}^{\prime} \cup e_{q}^{\prime}$ on $\Gamma_{a}$, and $e_{1} \cup e_{1}^{\prime}$ and $e_{p} \cup e_{q}^{\prime}$ on $\Gamma_{b}$. Then the union $A=D_{1} \cup \ldots \cup D_{4}$ is a Möbius band or annulus in $M$ with boundary on $T_{0}$. (It is embedded in $M$, otherwise there is a pair of edges parallel in both graphs, contradicting Lemma 2.2(2).) If $A$ is a Möbius band then it is already a contradiction to the hyperbolicity of $M$. If $A$ is an annulus and $p \neq q$ then a boundary component $c$ of $A$ has intersection number $p-q \neq 0$ with $\cup \partial v_{i}$ and hence is an essential curve on $T_{0}$. Since $e_{1}$ is an essential arc on both $A$ and $F_{1}$ and $F_{1}$ is boundary incompressible, $A$ cannot be boundary parallel. It follows that $A$ is an essential annulus in $M$, which again contradicts the assumption that $M$ is hyperbolic.

Lemma 2.20 Suppose $\Gamma_{b}$ is positive, $n_{b} \geq 3$, and $\Gamma_{a}$ contains bigons $e_{1} \cup e_{2}$ and $e_{1}^{\prime} \cup e_{2}^{\prime}$, such that $e_{1}, e_{1}^{\prime}$ have label pair $\{i, j\}$ and $e_{2}, e_{2}^{\prime}$ have label pair 
$\{i+1, j+1\}$, where $j \neq i$. Let $C_{1}=e_{1} \cup e_{1}^{\prime}$ and $C_{2}=e_{2} \cup e_{2}^{\prime}$ be the loops on $\hat{F}_{b}$. If $C_{1}$ is essential on $\hat{F}_{b}$ then $C_{2}$ is essential on $\hat{F}_{b}$ and not homotopic to $C_{1}$.

Proof. Let $B$ and $B^{\prime}$ be the bigon faces bounded by $e_{1} \cup e_{2}$ and $e_{1}^{\prime} \cup e_{2}^{\prime}$, respectively. Shrinking the Dehn filling solid torus to the core knot $K_{b}$, the union $B \cup B^{\prime}$ becomes an annulus $A_{1}$ in $M\left(r_{b}\right)$ with boundary $C_{1} \cup C_{2}$. Since $\hat{F}_{b}$ is incompressible and $C_{1}$ is essential on $\hat{F}_{b}$, it follows that $C_{2}$ must also be essential on $\hat{F}_{b}$.

Now assume $C_{i}$ are essential and homotopic on $\hat{F}_{b}$. Since $i \neq j$ and $n_{b}>2$, $C_{1}, C_{2}$ have at most one vertex in common. If $C_{1}, C_{2}$ are disjoint, let $A_{2}$ be an annulus on $\hat{F}_{b}$ bounded by $C_{1} \cup C_{2}$. If $C_{1}, C_{2}$ has a common vertex $v_{i+1}=v_{j}$, let $A_{2}$ be the disk face of $C_{1} \cup C_{2}$ in $\hat{F}_{b}$, which will be considered as a degenerate annulus as it can be obtained from an annulus by pinching an essential arc to a point. Let $A_{2}^{\prime}$ be the closure of $\hat{F}_{b}-A_{2}$. Let $m$ and $m^{\prime}$ be the number of vertices in the interior of $A_{2}$ and $A_{2}^{\prime}$, respectively. Then $n_{b}=m+m^{\prime}+k$, where $k$ is the number of vertices on $C_{1} \cup C_{2}$, i.e., $k=4$ if $C_{1} \cap C_{2}=\emptyset$, and $k=3$ otherwise.

First consider the case that $C_{1} \cap C_{2}=\emptyset$. Orient $C_{1}, C_{2}$ so that they are parallel on the annulus $A_{1}$. If they are also parallel on $\hat{F}_{b}$ then $A_{1} \cup A_{2}$ is a nonseparating torus which can be perturbed to intersect $K_{b}$ at $m+2<n$ points, which is a contradiction. If they are anti-parallel then $A_{1} \cup A_{2}$ and $A_{1} \cup A_{2}^{\prime}$ are Klein bottles which can be perturbed to intersect $K_{b}$ at $m$ and $m^{\prime}$ points, respectively. Since at least one of $m, m^{\prime}$ is less than $n_{b} / 2$, this contradicts Lemma 2.12.

The case that $C_{1} \cap C_{2} \neq \emptyset$ is similar. If $C_{1}, C_{2}$ are parallel then $A_{1} \cup$ $A_{2}$ is a torus and can be perturbed to intersect $K_{b}$ at $m+1<n$ points; if they are anti-parallel then $A_{1} \cup A_{2}$ and $A_{1} \cup A_{2}^{\prime}$ can be perturbed to be Klein bottles intersecting $K_{b}$ at $m+1$ and $m^{\prime}$ points, respectively, which leads to contradictions as above because $m+1+m^{\prime}<n_{b}$ implies either $2(m+1)<n_{b}$ or $2 m^{\prime}<n_{b}$.

A triple of edge endpoints $\left(p_{1}, p_{2}, p_{3}\right)$ on $\Gamma_{b}$ is positive if they appear on the boundary of the same vertex $v_{i}$, and in this order on $\partial v_{i}$ along the orientation of $\partial v_{i}$. Note that this is true if and only if $d_{v_{i}}\left(p_{1}, p_{2}\right)+d_{v_{i}}\left(p_{2}, p_{3}\right)=d_{v_{i}}\left(p_{1}, p_{3}\right)$.

Lemma 2.21 (1) Suppose $\left(p_{1}, p_{2}, p_{3}\right)$ is a positive triple on $\Gamma_{b}$. Let $k$ be a fixed integer and let $p_{i}^{\prime}$ be edge endpoints such that $d_{\Gamma_{a}}\left(p_{i}, p_{i}^{\prime}\right)=k$ for all $i$. Then $\left(p_{1}^{\prime}, p_{2}^{\prime}, p_{3}^{\prime}\right)$ is also a positive triple on $\Gamma_{b}$.

(2) Let $e_{1} \cup \ldots \cup e_{r}$ be a set of parallel negative edges with end vertices $u_{1}, u_{2}$ in $\Gamma_{a}$. Let $u(p) \in\left\{u_{1}, u_{2}\right\}$ for $p=1,2,3$, and let $e_{j}(u(p))$ be the endpoint of $e_{j}$ at $u(p)$. If $\left(e_{i}(u(1)), e_{j}(u(2)), e_{k}(u(3))\right)$ is a positive triple and $i, j, k \leq r-t$, then $\left(e_{i+t}(u(1)), e_{j+t}(u(2)), e_{k+t}(u(3))\right)$ is also a positive triple.

Proof. (1) Geometrically this is obvious: Flowing on $T_{0}$ along $\partial F_{a}$ moves the first triple to the second triple, hence the orientations of the components of $\partial F_{b}$ containing these triples are the same on $T_{0}$. 
Alternatively one may use Lemma 2.16(ii) to prove the result. Since $d_{\Gamma_{a}}\left(p_{i}, p_{i}^{\prime}\right)=$ $d_{\Gamma_{a}}\left(p_{j}, p_{j}^{\prime}\right)=k$ for all $i, j$, by Lemma 2.16 (ii) we have $d_{\Gamma_{b}}\left(p_{i}, p_{j}\right)=d_{\Gamma_{b}}\left(p_{i}^{\prime}, p_{j}^{\prime}\right)$ for all $i, j$. Therefore $d_{\Gamma_{b}}\left(p_{1}, p_{2}\right)+d_{\Gamma_{b}}\left(p_{2}, p_{3}\right)=d_{\Gamma_{b}}\left(p_{1}, p_{3}\right)$ if and only if $d_{\Gamma_{b}}\left(p_{1}^{\prime}, p_{2}^{\prime}\right)+d_{\Gamma_{b}}\left(p_{2}^{\prime}, p_{3}^{\prime}\right)=d_{\Gamma_{b}}\left(p_{1}^{\prime}, p_{3}^{\prime}\right)$.

(2) This is a special case of (1) because

$$
\begin{aligned}
d_{\Gamma_{a}}\left(e_{i}(u(1)), e_{i+t}(u(1))\right) & =d_{\Gamma_{a}}\left(e_{j}(u(2)), e_{j+t}(u(2))\right) \\
& =d_{\Gamma_{a}}\left(e_{k}(u(3)), e_{k+t}(u(3))\right)=t
\end{aligned}
$$

Lemma 2.22 Suppose $\Gamma_{b}$ is positive and $n=n_{b} \geq 3$.

(1) Suppose $\hat{e} \supset e_{1} \cup \ldots \cup e_{n+2}$, and the transition number $s=1$. Let $A$ be the annulus obtained by cutting $\hat{F}_{b}$ along the cycle $e_{1} \cup \ldots \cup e_{n}$. Then the edges $e_{n+1}, e_{n+2}$ lie in $A$ as shown in Figure 2.3, up to reflection along the center circle of the annulus.

(2) If $s_{1}=1, n=3$ and $\hat{e}_{1}$ contains 6 edges $e_{1} \cup \ldots \cup e_{6}$ then the edges are as shown in Figure 2.4.

(3) Any family of parallel negative edges in $\Gamma_{a}$ contains at most $2 n$ edges.

Proof. (1) Let $u, u^{\prime}$ be the end vertices of $\hat{e}$ in $\Gamma_{a}$. Orient $e_{i}$ from $u$ to $u^{\prime}$ and assume without loss of generality that $e_{i}$ has label $i$ at its tail $e_{i}(t)$ in $\Gamma_{a}$. Since $s=1$, the head of $e_{i}$, denoted by $e_{i}(h)$, has label $i+1$ in $\Gamma_{a}$. The edges $e_{1}, \ldots, e_{n}$ form an essential loop on the torus $\hat{F}_{b}$. Cutting $\hat{F}_{b}$ along this loop produces an annulus, as shown in Figure 2.3.

Up to reflection along the center circle of the annulus we may assume that the edge $e_{n+1}$ appears in this annulus as shown in Figure 2.3. We need to prove that $e_{n+2}$ appears in $\Gamma_{b}$ as shown in the figure. 


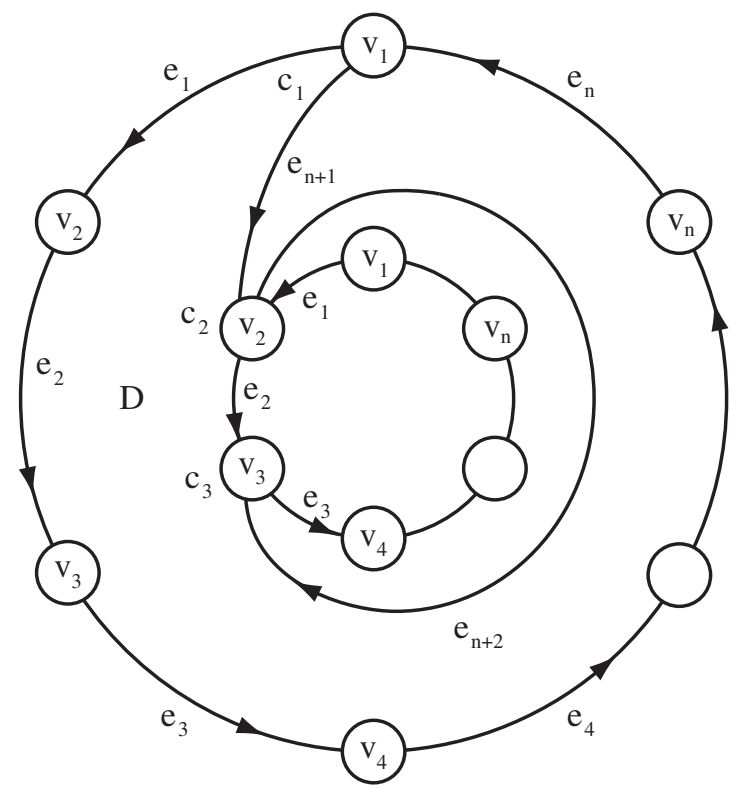

Figure 2.3

Since $\Gamma_{b}$ is positive, we may assume that all vertices on Figure 2.3 are oriented counterclockwise. Note that $\left(e_{1}(h), e_{n+1}(h), e_{2}(t)\right)$ is a positive triple on $\Gamma_{b}$. By Lemma 2.21(2) the triple $\left(e_{2}(h), e_{n+2}(h), e_{3}(t)\right)$ is also a positive triple. This determines the location of the head of $e_{n+2}$, as shown in Figure 2.3. Applying Lemma 2.20 to $e_{1} \cup e_{2}$ and $e_{n+1} \cup e_{n+2}$, we see that the loop $e_{2} \cup e_{n+2}$ is essential and not homotopic to $e_{1} \cup e_{n+1}$ on $F_{b}$, so these two loops must intersect transversely at the common vertex $v_{2}$ on $\hat{F}_{b}$. Hence the edge $e_{n+2}$ must appear as shown in Figure 2.3.

(2) By (1) the first 5 edges must be as shown in Figure 2.4. These cut the torus into a 3 -gon and a 7-gon. The edge $e_{6}$ is not parallel to the other $e_{i}$ 's on $\Gamma_{b}$ and hence must lie in the 7-gon, connecting $v_{3}$ to $v_{1}$. For the same reason as above, $\left(e_{3}(h), e_{6}(h), e_{4}(t)\right)$ is a positive triple on $\partial v_{1}$, hence the head of $e_{6}$ must be in the corner on $\partial v_{1}$ from $e_{1}(t)$ to $e_{4}(t)$ because the corner from $e_{3}(h)$ to $e_{1}(t)$ lies in the 3 -gon. Similarly, since $\left(e_{1}(h), e_{5}(t), e_{4}(h)\right)$ is a positive triple on Figure 2.4, by Lemma 2.21(2) $\left(e_{2}(h), e_{6}(t), e_{5}(h)\right)$ is also a positive triple, which determines the position of the tail of $e_{6}$. Therefore $e_{6}$ must be as shown in Figure 2.4.

(3) This follows from [Go, Corollary 5.5] when $n \geq 4$. Now assume $n=3$ and suppose there exist $2 n+1=7$ parallel edges $e_{1} \cup \ldots \cup e_{7}$ on $\Gamma_{a}$. By the 3 -Cycle Lemma 2.14(2) we may assume that the transition number $s \neq 0$. Since $n=3$, we may assume without loss of generality that $s=1$, hence by (2) the subgraph of $\Gamma_{b}$ consisting of the edges $e_{1} \cup \ldots \cup e_{6}$ is as shown in Figure 2.4. By the same argument as above, $\left(e_{4}(h), e_{7}(h), e_{5}(t)\right)$ and $\left(e_{3}(h), e_{7}(t), e_{6}(h)\right)$ are positive triples on $\partial v_{2}$ and $\partial v_{1}$, respectively. Since $e_{7}$ must lie in the 6- 
gon face $D$ in Figure 2.4, this is possible only if $e_{7}$ is parallel to $e_{1}$, which is a contradiction to Lemma 2.2(2).

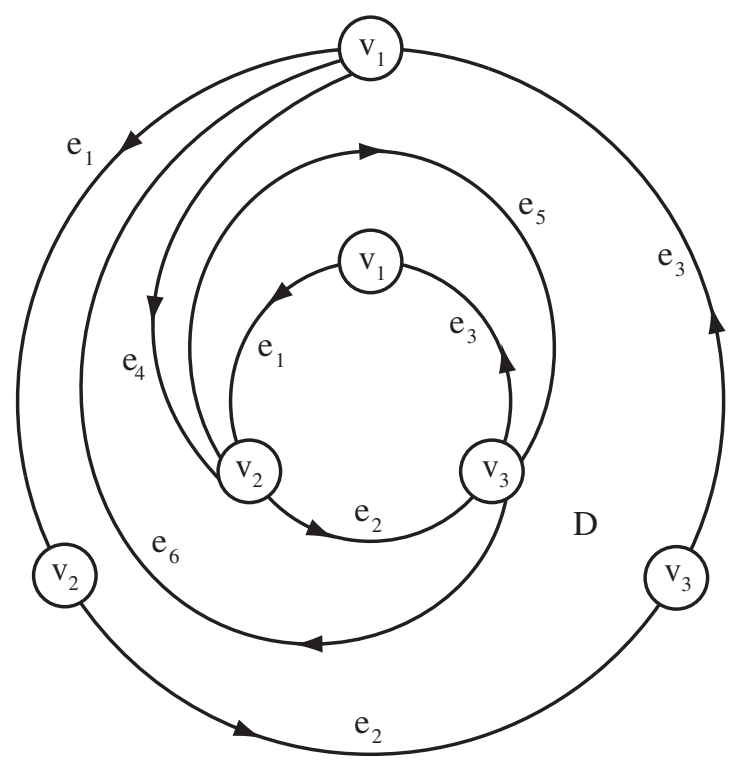

Figure 2.4

Lemma 2.23 Let sign $(v)$ be the sign of a vertex $v$, and define $p_{a}$ to be the sum of the signs of the vertices of $\Gamma_{a}$. Then either $p_{1}=0$ or $p_{2}=0$. In particular, $n_{1}, n_{2}$ cannot both be odd.

Proof. For each edge endpoint $c$ on $u_{i} \cap v_{j}$, define $\operatorname{sign}(c)=\operatorname{sign}\left(u_{i}\right) \operatorname{sign}\left(v_{j}\right)$. Then the parity rule says that the two endpoints of an edge $e$ have different sign. Summing over all edge endpoints on $\Gamma_{a}$ gives

$$
0=\sum_{i, j} \Delta\left(\operatorname{sign}\left(u_{i}\right) \operatorname{sign}\left(v_{j}\right)\right)=\Delta \sum_{i} \operatorname{sign}\left(u_{i}\right) \sum_{j} \operatorname{sign}\left(v_{j}\right)=\Delta p_{1} p_{2}
$$

hence either $p_{1}=0$ or $p_{2}=0$.

\section{$3 \quad \hat{\Gamma}_{a}^{+}$has no interior vertex}

In this section we will show that if $n_{b}>4$ then the graph $\Gamma_{a}$ does not have interior vertices; in particular the vertices of $\Gamma_{a}$ cannot all be parallel. Recall that we have assumed that $\Delta \geq 4$.

Lemma 3.1 If $n_{b}>4$ then $\hat{\Gamma}_{a}$ has no full vertex of valence at most 6 . 
Proof. First assume $n_{b} \neq 6$. Then by Lemma 2.7(3), three adjacent families of positive edges in $\Gamma_{a}$ contain at most $2 n_{b}-1$ edges, hence if $\Gamma_{a}$ has a full vertex of valence at most 6 then

$$
4 n_{b} \leq \Delta n_{b} \leq 2\left(2 n_{b}-1\right)=4 n_{b}-2,
$$

a contradiction.

So suppose $n_{b}=6$, and let $u_{1}$ be a full vertex of $\hat{\Gamma}_{a}$ of valence at most 6 . By Lemma 2.3(3), each family of edges incident to $u_{1}$ contains at most 4 edges. Since there are at least 24 edges in at most 6 families, there must be exactly 6 families, each containing exactly 4 edges. Each family contains a Scharlemann bigon, so there are six Scharlemann bigons at the vertex $u_{1}$. Since there are no extended Scharlemann cycles, each Scharlemann bigon appears at one end of a family of parallel edges. Thus by examining the labels around the vertex $u_{1}$, one can see that if one Scharlemann bigon has label pair $\{1,2\}$ then the others must have label pair $\{1,2\},\{3,4\}$ or $\{5,6\}$, and that at least two pairs do occur as label pairs of Scharlemann bigons. On the other hand, by Lemma 2.3(4) all three pairs cannot appear as label pairs of Scharlemann bigons. Hence, without loss of generality, there are incident to $u_{1}$ at least three (12)-Scharlemann bigons and a (34)-Scharlemann bigon. Since on $\Gamma_{b}$ the edges of the (34)-Scharlemann bigon form an essential loop on $\hat{\Gamma}_{b}$, there are at most two edges of $\hat{F}_{b}$ joining $v_{1}$ to $v_{2}$. Since the three (12)-Scharlemann bigons give rise to six negative 1-edges of $\Gamma_{b}$ joining $v_{1}$ to $v_{2}$, three of these must be parallel, contradicting Lemma $2.7(1)$.

Lemma 3.2 If $n_{b}>4$ then $\hat{\Gamma}_{a}^{+}$has no interior vertices.

Proof. This follows from Lemma 3.1 if $n_{a} \leq 2$ because in this case either $\hat{\Gamma}_{a}^{+}=\hat{\Gamma}_{a}$ and there is a full vertex of valence at most 6 , or $n_{a}=2$ and there is no interior vertex. Therefore we may assume that $n_{a} \geq 3$.

Suppose to the contrary that $\hat{\Gamma}_{a}^{+}$has an interior vertex $u_{i}$. By Lemma 3.1 all interior vertices of $\hat{\Gamma}_{a}^{+}$have valence at least 7 , hence we can apply Lemma 2.11 to conclude that $\hat{\Gamma}_{a}^{+}$has a boundary vertex $u_{1}$ of valence at most 3 .

By Lemma 2.7(3) the three families of adjacent positive edges at $u_{1}$ contain at most $2 n_{b}$ edges, hence there are $2 n_{b}$ adjacent negative edges. On $\Gamma_{b}$ this implies that each vertex $v_{j}$ is incident to two positive edges with label 1 at $v_{j}$, which cannot be parallel as otherwise there would be at least $n_{a}+1>n_{a} / 2+2$ parallel positive edges, contradicting Lemma 2.3(3). Therefore the reduced graph $\hat{\Gamma}_{b}$ contains at least $n_{b}$ positive edges. On the other hand, the existence of an interior vertex in $\hat{\Gamma}_{a}^{+}$implies that $\hat{\Gamma}_{b}$ contains at least $2 n_{b}$ negative edges, as shown in the proof of Lemma 3.1. Since $\hat{\Gamma}_{b}$ has at most $3 n_{b}$ edges (Lemma 2.5), it must have exactly $n_{b}$ positive edges and $2 n_{b}$ negative edges. Since we have shown above that each vertex in $\hat{\Gamma}_{b}$ is incident to at least two positive edges, it follows that it is incident to exactly two positive edges.

We claim that a family of parallel positive edges in $\Gamma_{b}$ contains at most $n_{a} / 2$ edges. If such a family contains more than $n_{a} / 2$ edges, then there is 
a Scharlemann bigon on one side of the family, and by looking at the labels one can see that all labels appear among the endpoints of this family, which is impossible because $u_{i}$ being an interior vertex in $\hat{\Gamma}_{a}^{+}$implies that all edges in $\Gamma_{b}$ with $i$ as a label are negative.

Since each vertex $v_{j}$ is incident to two families of positive edges, each containing at most $n_{a} / 2$ edges, we see that $v_{j}$ is incident to at least $3 n_{a}$ negative edges. By Lemmas 3.1 and 2.5 we see that $\hat{\Gamma}_{a}$ has less than $3 n_{a}$ positive edges, hence two of the negative edges incident to $v_{j}$ are parallel in $\Gamma_{a}$, so $j$ is a label of a Scharlemann bigon in $\Gamma_{a}$. Since this is true for all vertices in $\Gamma_{b}$, by Lemma $2.3(4)$ we have $n_{b} \leq 4$, which is a contradiction.

\section{Possible components of $\hat{\Gamma}_{a}^{+}$}

Lemma 4.1 Suppose $\hat{\Gamma}_{a}^{+}$has no isolated vertex or interior vertex. If some $v_{i}$ of $\Gamma_{b}$ is incident to more than $2 n_{a}$ negative edges in $\Gamma_{b}$, or if $n_{a}>4$ and $v_{i}$ is incident to at most two families of positive edges in $\Gamma_{b}$, then $i$ is a label of a Scharlemann bigon in $\Gamma_{a}$.

Proof. If $v_{i}$ is a vertex of $\Gamma_{b}$ incident to more than $2 n_{a}$ negative edges then by Lemma 2.10(2) two of them are parallel in $\Gamma_{a}$, so by Lemma 2.4 they form a Scharlemann bigon, hence $i$ is the label of a Scharlemann bigon in $\Gamma_{a}$. If $n_{a}>4$ and $v_{i}$ is incident to two families of positive edges in $\Gamma_{b}$ then by Lemma 2.3(3) each family contains less than $n_{a}$ edges, hence $v_{i}$ is incident to more than $2 n_{a}$ negative edges and the result follows from the above.

In the rest of this section we assume $n_{a}>4$ for $a=1,2$, and $\Delta \geq 4$. By Lemma $3.2 \hat{\Gamma}_{a}^{+}$has no interior vertices. We will show that each component of $\hat{\Gamma}_{a}^{+}$must be one of the 11 graphs in Figure 4.2.

Lemma 4.2 No vertex $u$ of $\hat{\Gamma}_{a}$ is incident to at most four positive edges and at most one negative edge.

Proof. By Lemmas 2.7(1) and 2.7(2) a family of negative edges contains at most $n_{b}$ edges, and four adjacent families of positive edges contain at most $2\left(n_{b}+2\right)=2 n_{b}+4$ edges. Since $\Gamma_{a}$ has at least $4 n_{b}$ edges incident to $u$, we would have $n_{b} \leq 4$, which is a contradiction to our assumption.

Lemma 4.3 Suppose $u_{i}$ is incident to at most three positive edges in $\hat{\Gamma}_{a}$, and if there are three then two of them are adjacent. Then $i$ is a label of a Scharlemann bigon in $\Gamma_{b}$.

Proof. In this case each label appears at the endpoint of some negative edge at $u_{i}$, so $\hat{\Gamma}_{b}^{+}$has no isolated vertex. By Lemma 4.1 the result is true if $u_{i}$ is incident to more than $2 n_{b}$ negative edges. So we assume that $u_{i}$ is incident to no more than $2 n_{b}$ negative edges, and hence at least $2 n_{b}$ positive edges. By 
Lemma 2.7(2) the two adjacent families of positive edges contain at most $n_{b}+2$ edges, while the other positive family contains no more than $n_{b} / 2+2$ edges. Thus $\left(n_{b}+2\right)+\left(n_{b} / 2+2\right) \geq 2 n_{b}$, which gives $n_{b} \leq 8$. Since one of the positive families contains more than $n_{b} / 2$ edges, it contains a Scharlemann bigon; by Lemma 2.2(4) $n_{b}$ must be even, so $n_{b}=8$ or 6 . Using the above inequality and the fact that when $n_{b}=6$ each positive family contains at most 4 edges (Lemma $2.3(3)$ ), we see that $u_{i}$ is incident to exactly $2 n_{b}$ positive edges and $2 n_{b}$ negative edges. Dually, this implies that in $\Gamma_{b}$ there are exactly $2 n_{b}$ positive $i$-edges and $2 n_{b}$ negative $i$-edges. (As always, an edge with both endpoints labeled $i$ is counted twice.)

If $i$ is not a label of a Scharlemann bigon in $\Gamma_{b}$ then the $2 n_{b}$ positive $i$ edges in $\Gamma_{b}$ are mutually nonparallel, so $\hat{\Gamma}_{b}$ has at least $2 n_{b}$ positive edges. By Lemma 2.5 the reduced graph $\hat{\Gamma}_{b}$ has no more than $3 n_{b}$ edges, so it has at most $n_{b}$ negative edges. On the other hand, by Lemma 2.7(1) each family of parallel negative edges in $\Gamma_{b}$ has at most two endpoints labeled $i$; since there are $2 n_{b}$ such endpoints, $\Gamma_{b}$ must have at least $n_{b}$ families of negative edges. It follows that $\Gamma_{b}$ has exactly $n_{b}$ families of negative edges, each having exactly two endpoints labeled $i$.

Suppose $n_{b}=6$. Then there are 12 edges in the three families incident to $u_{i}$, and by Lemma 2.3(3) each family contains at most four edges, hence each family contains exactly four edges. If some of these edges are loops, then there are four loops and four non-loop edges. No loop can be parallel to a non-loop edge in $\Gamma_{b}$ since otherwise the label $i$ would appear three times among a set of parallel edges in $\Gamma_{b}$. It follows that all the 8 positive edges incident to $u_{i}$ are mutually nonparallel in $\Gamma_{b}$, so the reduced graph $\hat{\Gamma}_{b}$ would have at least 8 negative edges, which is a contradiction as we have shown above that $\hat{\Gamma}_{b}$ has exactly $n_{b}=6$ negative edges. Hence we can assume there is no loop based at $u_{i}$. Note that a family of four parallel edges in $\Gamma_{a}$ contains a Scharlemann bigon. If the label pair of the Scharlemann bigon is $\{j, j+1\}$, then these two labels appear twice among the endpoints of this family, and each of the other four labels appears exactly once. By Lemma 2.3(4) at most four labels are the labels of some Scharlemann bigons in $\Gamma_{b}$, so there is some $k$ which is not a label of a Scharlemann bigon and hence appears exactly three times among the endpoints of the positive edges incident to $u_{i}$. Dually, this implies that some negative edge in $\hat{\Gamma}_{b}$ contains only one $i$-edge, which is a contradiction as we have shown above that each negative edge in $\hat{\Gamma}_{b}$ must contain exactly two negative $i$-edges.

The proof for $n_{b}=8$ is similar. In this case the numbers of edges in the three positive families incident to $u_{i}$ are either $(6,5,5)$ or $(6,6,4)$. Using the fact that there are at most four labels of Scharlemann cycles one can show that in either case some label appears three times among the endpoints of these edges, which would lead to a contradiction as above.

Lemma 4.4 No vertex $u_{i}$ is incident to at most one edge in $\hat{\Gamma}_{a}^{+}$.

Proof. By Lemma 3.2 there are no interior vertices, hence by Lemma 2.11 either (i) $\hat{\Gamma}_{b}^{+}$has a circle component, or (ii) $\hat{\Gamma}_{b}^{+}$has a boundary vertex of valence at 
most 3, or (iii) all vertices of $\hat{\Gamma}_{b}^{+}$are boundary vertices of valence 4 .

In case (i) a vertex $v_{j}$ on the circle component is incident to at most two positive edges with label $i$ at $v_{j}$, hence dually there are at most two negative edges with label $j$ at $u_{i}$, and hence at least $\Delta-2 \geq 2$ positive edges with label $j$ at $u_{i}$, which is impossible because $u_{i}$ is incident to at most one family of positive edges and by Lemma 2.3(3) such a family contains at most one edge with label $j$ at $u_{i}$.

The proof for case (ii) is similar because by Lemma 2.7(3) a valence 3 boundary vertex $v_{j}$ of $\hat{\Gamma}_{b}^{+}$is incident to at most $2 n_{a}$ positive edges of $\Gamma_{b}$ and hence at most two positive edges with label $i$ at $v_{j}$.

In case (iii), since $u_{i}$ is incident to at most $n_{b} / 2+2<n_{b}$ positive edges, there is a label $j$ such that all four edges with label $j$ at $u_{i}$ are negative. Dually $v_{j}$ has four positive $i$-edges. Since it is a boundary vertex, it is incident to at least $3 n_{a}+1$ positive edges. On the other hand, since $v_{j}$ has valence 4 in $\hat{\Gamma}_{b}^{+}$, by Lemma $2.7(2)$ it has at most $2\left(n_{a}+2\right)<3 n_{a}$ positive edges, a contradiction.

Corollary 4.5 Each component of $\hat{\Gamma}_{a}^{+}$is contained in an essential annulus but not a disk on $\hat{F}_{a}$.

Proof. By Lemma 2.6 $\hat{\Gamma}_{a}^{+}$has at least two components, so if the result is not true then one can find a disk $D$ on $\hat{F}_{a}$ such that $D \cap \hat{\Gamma}_{a}^{+}$is a component $G$ of $\hat{\Gamma}_{a}^{+}$. By Lemma $4.4 G$ is not an arc, so by Lemma 2.9 it has at least three boundary vertices of valence at most 3. By Lemma 4.3 these vertices are labels of Scharlemann cycles in $\Gamma_{b}$, which is a contradiction because by Lemma 2.3(4) $\Gamma_{a}$ contains at most two labels of Scharlemann cycles of each sign.

Let $G$ be a component of $\hat{\Gamma}_{a}^{+}$contained in the interior of an essential annulus $A$ on $\hat{F}_{a}$. By Corollary 4.5, $G$ is not contained in a disk, hence it contains some cycles which are topologically essential simple closed curves on $\hat{F}_{a}$, and all such cycles are isotopic to the core of $A$. We call such a cycle an essential cycle on $G$. Note that a cycle may have more than two edges incident to a vertex, but an essential cycle does not. An essential cycle $C$ of $G$ is outermost on $A$ if all essential cycles of $G$ lie in one component of $A \mid C$. By cutting and pasting one can see that outermost essential cycles always exist, and there are at most two of them, which we denote by $C_{l}$ and $C_{r}$, called the leftmost cycle and the rightmost cycle, respectively. Let $A_{l}^{l}$ and $A_{l}^{r}$ be the components of $A \mid C_{l}$, called the left annulus and the right annulus of $C_{l}$, respectively, labeled so that $A_{l}^{l}$ contains no essential cycles of $G$ other than $C_{l}$. Similarly for $A_{r}^{l}$ and $A_{r}^{r}$, where the right annulus $A_{r}^{r}$ of $C_{r}$ is the one that contains no essential cycles other than $C_{r}$.

Lemma 4.6 The interiors of $A_{l}^{l}$ and $A_{r}^{r}$ do not intersect $G$.

Proof. Assuming the contrary, let $G^{\prime}$ be the closure of a component of $G \cap A_{l}^{l}$. Since $G$ is connected, $G^{\prime}$ must intersect $C_{l}$ at some vertex $v$, but it cannot intersect $C_{l}$ at more than one vertex, as otherwise the union of an arc in $G^{\prime}$ 
and an arc on $C_{l}$ would be an essential cycle in $A_{l}^{l}$ other than $C_{l}$, contradicting the definitions of leftmost cycle and its left annulus. For the same reason, $G^{\prime}$ contains no essential cycles, hence it lies on a disk $D$ in $A_{l}^{l}$. By Lemma $4.4 G$ has no vertex of valence 1 , so $G^{\prime}$ is not homeomorphic to an arc. By Lemma $2.9 G^{\prime}$ has at least three boundary vertices of valence at most 3 . Let $v^{1}$ and $v^{2}$ be such vertices other than $v$. They are boundary vertices of $G$ lying in the interior of $A_{l}^{l}$ with valence at most 3 , and $v^{i} \neq v$.

By Lemma 4.3, for $i=1,2$ there is a Scharlemann bigon $\left\{e_{1}^{i}, e_{2}^{i}\right\}$ on $\Gamma_{b}$ with $v^{i}$ as a label, and by Lemma 2.2(5) $C_{i}=e_{1}^{i} \cup e_{2}^{i}$ is an essential curve on $\hat{F}_{a}$ containing $v^{i}$. Since $v^{2}$ is a boundary vertex of $G^{\prime}$, it is not a cut vertex, hence there is an arc $C^{\prime}$ on $G^{\prime}$ connecting $v^{1}$ to $v$ which is disjoint from $v^{2}$. Now the union $C_{1} \cup C^{\prime} \cup C_{l}$ cuts $\hat{F}_{a}$ into an annulus and a disk $D$ containing $v^{2}$ in its interior, so the cycle $C_{2}$ is also contained in the disk $D$, which is a contradiction to the fact that $C_{2}$ is topologically an essential curve on $\hat{F}_{a}$.

Lemma 4.6 shows that $G$ is contained in the region $R$ between $C_{l}$ and $C_{r}$. Since $G$ has no interior vertices, all its vertices are on $C_{l} \cup C_{r}$. If $C_{l}$ is disjoint from $C_{r}$ then $R$ is an annulus, and if $C_{l}=C_{r}$ then $R=C_{l}=C_{r}$ is a circle. In the generic case we have $C_{l} \cap C_{r}=E_{1} \cup \ldots \cup E_{k}$, where each $E_{i}$ is either a vertex or an arc. The region $R$ is then a union of these $E_{i}$ and some disks $D_{1}, \ldots, D_{k}$, such that $\partial D_{i}$ is the union of two arcs, one in each of $C_{r}$ and $C_{l}$. When $k=1$ and $E_{1}=v$ is a vertex, $D_{1}$ is a disk with a pair of boundary points identified to the single point $v$. Note that a vertex of $G$ is a boundary vertex if and only if it is on $C_{l} \cup C_{r}-C_{l} \cap C_{r}$.

Lemma 4.7 Let $C=C_{l}$ or $C_{r}$.

(1) If $C$ has a boundary vertex $u_{i}$ of valence at most 3 then it has no other boundary vertex of valence at most 4.

(2) If $C$ has a boundary vertex $u_{i}$ of valence 2 then it has no other boundary vertex.

Proof. (1) By Lemma 4.3, $i$ is a label of a Scharlemann bigon in $\Gamma_{b}$. On $\Gamma_{a}$ the edges of this Scharlemann bigon form a cycle $C^{\prime}$ containing $u_{i}$ and another vertex $u_{k}$. By Lemma 2.2(5) $C^{\prime}$ is topologically an essential circle on the torus $\hat{F}_{a}$. Since $u_{i}$ is a boundary vertex, one can see that $C^{\prime}$ is topologically isotopic to $C$. By Lemma 4.6 applied to $G$ and to the component of $\hat{\Gamma}_{a}^{+}$containing $u_{k}$, there are no other vertices of $\Gamma_{a}$ between $C^{\prime}$ and $C$. Hence any boundary vertex $u_{j} \neq u_{i}$ on $C$ is incident to at at most one family of parallel negative edges, connecting it to $u_{k}$. The result now follows from Lemma 4.2.

(2) Note that since $u_{i}$ is a boundary vertex, the edges of any Scharlemann bigon on $\Gamma_{b}$ with $i$ as a label must connect $u_{i}$ to the same vertex $u_{k}$ on $\Gamma_{a}$, so there are at most $n_{b}$ such bigons because there are only two edges on $\hat{\Gamma}_{a}$ connecting $u_{i}$ to $u_{k}$, each representing a family of at most $n_{b}$ edges. By Lemma $2.7(2) u_{i}$ is incident to at most $n_{b}+2$ positive edges, hence at least $3 n_{b}-2$ negative edges. If a pair of these edges are parallel on $\Gamma_{b}$ then they form a Scharlemann bigon. Hence by the above we see that there are at most $n_{b}$ pairs 
of such edges. It follows that $\hat{\Gamma}_{b}$ has at least $3 n_{b}-2-n_{b}=2 n_{b}-2$ positive edges.

If $u_{j}$ is a boundary vertex of $C$ other than $u_{i}$ then as in the proof of (1) it is incident to at most one family of negative edges, so it has at least $3 n_{b}$ positive edges. Since no three of those are parallel on $\Gamma_{b}$, we see that $\hat{\Gamma}_{b}$ has at least $3 n_{b} / 2$ negative edges, so $\hat{\Gamma}_{b}$ would have a total of at least $2 n_{b}-2+3 n_{b} / 2>3 n_{b}$ edges, contradicting Lemma 2.5.

Now suppose $C_{l} \cap C_{r} \neq \emptyset$, and $C_{l} \neq C_{r}$. Then the region $R$ between $C_{l}$ and $C_{r}$ can be cut along vertices of $C_{l} \cap C_{r}$ to obtain a set of disks, and possibly some arcs. Let $D$ be such a disk. If $C_{l} \cap C_{r}$ is a single vertex $v$ then $D$ is obtained by cutting $R$ along $v$, in which case we use $D \cap G$ to denote the graph on $D$ obtained by cutting $G$ along $v$.
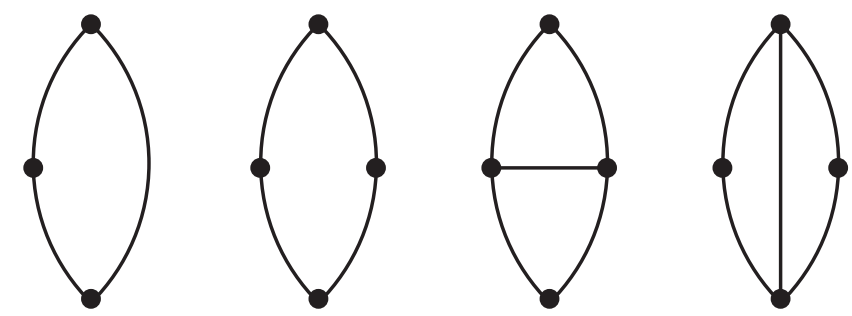

Figure 4.1

Lemma $4.8 G^{\prime}=D \cap G$ is one of the four graphs in Figure 4.1.

Proof. Let $v^{\prime}, v^{\prime \prime}$ be the vertices of $G^{\prime}$ lying on both $C_{l}$ and $C_{r}$. (Note that they are distinct vertices on $G^{\prime}$ but may be identified to a single vertex on $G$.) These vertices divide $\partial D$ into two $\operatorname{arcs} E_{1}$ and $E_{2}$, with $E_{1} \subset C_{l}$ and $E_{2} \subset C_{r}$.

By Lemma 4.7 , each $E_{j}$ contains at most one vertex of valence at most 3 in its interior. Therefore, if $D$ contains at most one interior edge then $G^{\prime}$ has at most four vertices, so it is one of the four graphs in Figure 4.1. We need to show that $D$ cannot have more than one interior edge.

First suppose there is an interior edge $e$ of $G^{\prime}$ which has both endpoints on $E_{1}$. We may choose $e$ to be outermost in the sense that there is an arc $E^{\prime}$ on $E_{1}$ with $\partial E^{\prime}=\partial e$, and there is no edge of $G^{\prime}$ inside the disk bounded by $E^{\prime} \cup e$. Since $G^{\prime}$ has no parallel edges, there must be a vertex $v$ in the interior of $E^{\prime}$, which has valence 2. By Lemma 4.7(2), in this case $C_{l}$ has no other boundary vertices, so $E_{1}$ has no vertex other than $v$ in its interior; in particular, $e$ must have its endpoints on $v^{\prime}$ and $v^{\prime \prime}$. This implies that all interior edges have both endpoints on $E_{2}$, and by the same argument as above we see that $E_{2}$ has exactly one vertex in its interior, and all edges must have endpoints on $v^{\prime}$ and $v^{\prime \prime}$. Since $G^{\prime}$ has no parallel edges, it can have at most one edge connecting $v^{\prime}$ to $v^{\prime \prime}$, and we are done. 
We can now assume that every interior edge of $G^{\prime}$ has one endpoint in the interior of each $E_{i}$. Let $G^{\prime \prime}$ be the union of the interior edges. The above implies that $G^{\prime \prime}$ cannot have a cycle, so it is a union of several trees with endpoints in the interiors of $E_{1}$ and $E_{2}$. A vertex of valence 1 in $G^{\prime \prime}$ is a vertex of valence 3 in $G^{\prime}$, and by Lemma 4.7(1) there is at most one such for each $E_{i}$. Therefore $G^{\prime \prime}$ is a chain, with two vertices of valence 1 and $k \geq 0$ vertices of valence 2 , so $G^{\prime}$ has one vertex of valence 3 on each $E_{i}$, and $k$ vertices of valence 4 . Note that these are boundary vertices. However, by Lemma 4.7(1), if $G$ has a vertex of valence 3 on $C_{l}$ then it has no boundary vertex of valence at most 4 on $C_{l}$, and similarly for $C_{r}$. It follows that $k=0$, which again implies that $G^{\prime}$ has only one interior edge.

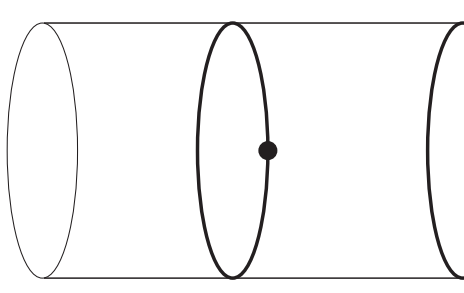

(1)
(5)

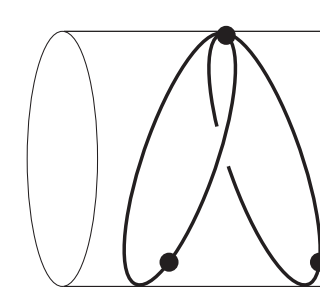

(2)

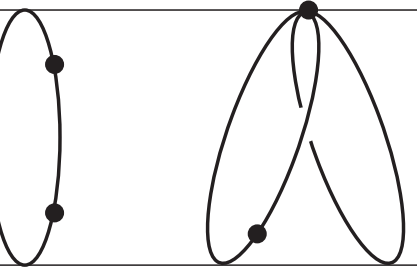

(3)

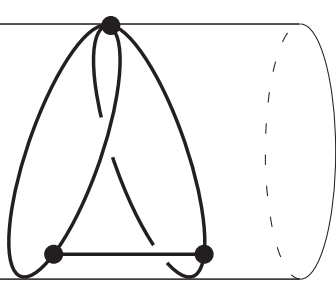

(4)

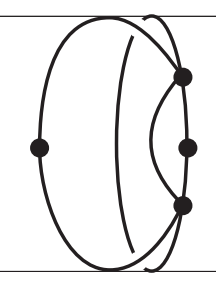

(6)

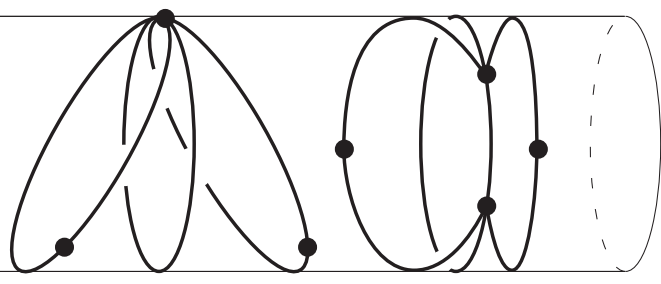

(7)

(8)

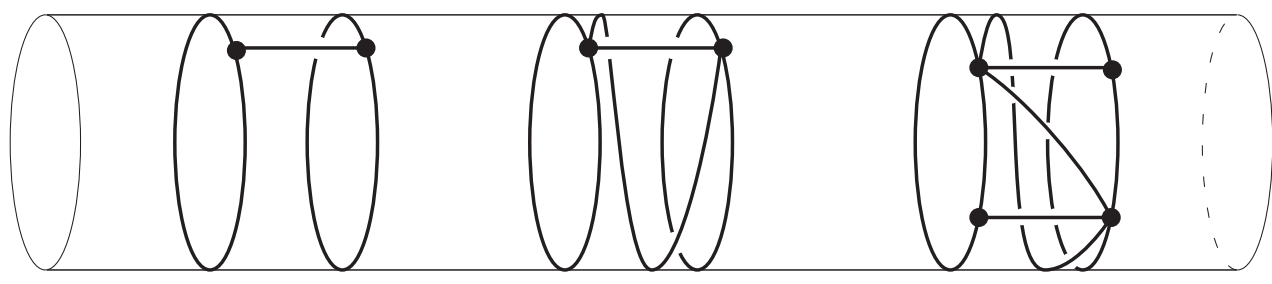

(9)
(11)

Figure 4.2

Lemma 4.9 If $G$ is a component of $\hat{\Gamma}_{a}$ and $C_{l} \cap C_{r} \neq \emptyset$, then $G$ is one of the graphs in Figure 4.2 (1) - (8). 
Proof. If $C_{l}=C_{r}$ then $G$ is a simple cycle, in which case each vertex has valence 2 and hence is a label of a Scharlemann bigon by Lemma 4.3. By Lemma 2.3(4), $G$ has at most two such vertices, hence $G$ is the graph in Figure 4.2(1) or (2).

Suppose $C_{l} \neq C_{r}$ and $C_{l} \cap C_{r} \neq \emptyset$. We call the endpoints of $C_{l} \cap C_{r}$ breaking points of $G$, which cut the region $R$ between $C_{l}$ and $C_{r}$ into several disks $D_{1}, \ldots, D_{k}$ and possibly some arcs. By Lemma 4.8 each $G_{i}=D_{i} \cap G$ is one of the graphs in Figure 4.1. We say that $G_{i}$ is of type $(j)$ if it is the graph in Figure 4.1(j). Since $G$ can have at most two boundary vertices of valence at most three, we see that either $k=1$, or $k=2$ and both $G_{i}$ are of type (1).

First assume that $k=2$ and $G_{1}, G_{2}$ are of type (1). By Lemma 4.7 the two boundary vertices of $G_{i}$ must be one on each of $C_{l}, C_{r}$. If the component of $C_{l} \cap C_{r}$ containing a breaking point $v^{\prime}$ on $G_{i}$ is an arc instead of a vertex, then $v^{\prime}$ would be a vertex of $\hat{\Gamma}_{b}$ which is incident to three positive edges, two of which are adjacent, in which case by Lemma $4.3 v^{\prime}$ is a label of a Scharlemann bigon in $\Gamma_{b}$. Since $G$ contains no more than two Scharlemann bigon labels, this cannot happen. It follows that $G$ is the graph shown in Figure 4.2(6).

We can now assume $k=1$. For the same reason as above, we see that if $G_{1}$ is of type (1), (2) or (3), then $G$ is as shown in Figure 4.2(3), (5) or (4), respectively. If $G_{1}$ is of type (4), the breaking vertices may be incident to an edge in $C_{l} \cap C_{r}$, so $G$ is the graph in Figure 4.2(7) or (8).

Lemma 4.10 If $G$ is a component of $\hat{\Gamma}_{a}^{+}$and $C_{l} \cap C_{r}=\emptyset$, then $G$ is one of the three graphs in Figure 4.2 (9), (10), or (11).

Proof. Note that in this case all vertices on $C_{l}$ and $C_{r}$ are boundary vertices. If $C_{l}$ has a vertex of valence 2 then by Lemma 4.7 it has no other vertices, in which case $C_{l}$ is a loop and we have $G=C_{l}$, so $C_{l}=C_{r}$, a contradiction. Therefore $C_{l}$ and $C_{r}$ have no vertices of valence 2 , hence all vertices of $G$ have valence at least 3 .

Doubling the annulus and calculating Euler characteristic, we see that

$$
\sum\left(4-\operatorname{val}\left(v_{i}\right)\right) \geq 0 .
$$

By Lemma $4.7 G$ has at most two vertices of valence 3 .

First assume that $G$ has two vertices $v_{1}, v_{2}$ of valence 3. By Lemma 4.7 $v_{1}, v_{2}$ cannot both be on $C_{l}$ or $C_{r}$, hence each of $C_{l}$ and $C_{r}$ contains exactly one vertex of valence 3 . By Lemma 4.7 they cannot contain vertices of valence 4 . By the above formula $G$ has either (i) no other vertex, or (ii) one other vertex with valence 6 or 5 , or (iii) two other vertices, both having valence 5 . One can check that in Case (i) the graph is that of Figure 4.2(9), Case (ii) does not happen, and in Case (iii) the graph is the one in Figure 4.2(11).

If $G$ contains only one vertex $v$ of valence 3 , then by the above formula it contains at least one vertex of valence 5 , and all other vertices are of valence 4 . If $C_{l}$ contains $v$ then by Lemma 4.7 it contains no vertices of valence 4 . Since each edge of $G$ must have one endpoint on each of $C_{l}$ and $C_{r}$, we see that $C_{l}$ must contain a vertex of valence 5 , and $C_{r}$ contains exactly two vertices, each 
of valence 4 . One can check that there is no reduced graph satisfying these conditions.

Now assume that $G$ has no vertices of valence 3 . Then by the above formula all vertices of $G$ are of valence 4 . Since $G$ has no parallel edges, all edges of $G-C_{l} \cup C_{r}$ must connect $C_{r}$ to $C_{l}$, so the graph $G$ is completely determined by the number of vertices $k$ on $C_{r}$, which must be the same as that on $C_{l}$. Denote such a graph by $G_{k}$. When $k=1$, the graph $G=G_{1}$ is shown in Figure 4.2(10). We need to show that $k>1$ does not happen.

Suppose $k>1$. By Lemma 4.2 each vertex on $C_{l}$ is incident to at least two negative edges in $\hat{\Gamma}_{a}$. Let $G^{\prime}$ be the component of $\hat{\Gamma}_{a}^{+}$adjacent to $C_{l}$, and let $C_{r}^{\prime}$ be the outermost cycle of $G^{\prime}$ adjacent to $C_{l}$. If $C_{r}^{\prime}$ has only one vertex then two negative edges based at some vertex $v_{i}$ on $C_{l}$ form an essential loop on the annulus between $C_{r}^{\prime}$ and $C_{l}$, so there is only one negative edge of $\hat{\Gamma}_{a}$ incident to any other vertex on $C_{l}$, which is a contradiction. Similarly if some vertex on $C_{r}^{\prime}$ is a boundary vertex of valence at most 3 then by Lemma 4.3 it is a label of a Scharlemann bigon, which is again a contradiction because the two edges of the Scharlemann bigon would form an essential loop as above. This rules out the possibility of $G^{\prime}$ being a graph in Figure 4.2 (1) or (3) - (11). If $G^{\prime}$ is the one in Figure 4.2(2) then by Lemma 4.3 both of its vertices are labels of Scharlemann bigons. The edges of these two Scharlemann bigons form two cycles, which cannot be on the same side of $G^{\prime}$ as otherwise one of them would lie on a disk, which contradicts Lemma 2.2(5). Hence one of the pairs of edges connect a vertex of $G^{\prime}$ to a vertex of $C_{l}$, which is again a contradiction.

It now follows that if some component of $\hat{\Gamma}_{a}^{+}$is a $G_{k}$ for $k \geq 2$, then so are all the other components. Moreover, none of the vertices is a label of a Scharlemann cycle as otherwise some vertex would be incident to a single negative edge in $\hat{\Gamma}_{a}$, which would contradict Lemma 4.2 . Hence $\Gamma_{b}$ has no Scharlemann cycles. On the other hand, by Lemma 2.7(2) the four families of positive edges at a vertex $v_{i}$ of $G_{k}$ contain at most $2 n_{b}+4$ edges, so $v_{i}$ is incident to at least $\Delta n_{b}-\left(2 n_{b}+2\right)>n_{b}$ negative edges. By Lemma 2.8 this implies that $\Gamma_{b}$ does have a Scharlemann cycle, which is a contradiction.

Corollary 4.11 Suppose $\Delta \geq 4$, and $n_{a}>4$ for $a=1,2$. Then

(1) each component of $\hat{\Gamma}_{a}^{+}$is one of the 11 graphs in Figure 4.2; and

(2) each $\Gamma_{a}$ contains a Scharlemann cycle, hence $\hat{F}_{b}$ is separating, and $n_{b}$ is even for $b=1,2$.

Proof. (1) This follows from Lemmas 4.9 and 4.10.

(2) By (1), $\hat{\Gamma}_{a}^{+}$contains either a vertex $v$ of valence 2 or a boundary vertex of valence at most 4 . In the first case the result follows from Lemma 4.3. In the second case by Lemma 2.7(2) $v$ is incident to at most $2 n_{b}+4$ positive edges, hence at least $2 n_{b}-4>n_{b}$ negative edges, so by Lemma $2.8 \Gamma_{a}$ has a Scharlemann cycle. 


\section{The case $n_{1}, n_{2}>4$}

In this section we will complete the proof that the generic case $n_{1}, n_{2}>4$ cannot happen. We assume throughout the rest of the section that $n_{1}, n_{2}>4$. Let $G$ be a component of $\hat{\Gamma}_{a}^{+}$. By Corollary $4.11 G$ is one of the graphs in Figure 4.2. We need to rule out all these possibilities. Recall that a component of $\hat{\Gamma}_{a}^{+}$is of type $(\mathrm{k})$ if it is the graph in Figure $4.2(\mathrm{k})$.

Here is a sketch of the proof. We first show (Lemma 5.4) that $\hat{\Gamma}_{a}^{+}$cannot have two boundary vertices of valence 2 , hence no component of $\hat{\Gamma}_{a}^{+}$is of type (5)-(8). Types (3) and (11) will be ruled out in Lemmas 5.6 and 5.7, so we are left with types $(1),(2),(4),(9)$ and (10). Lemma 5.8 will show that each vertex of a type (10) component is a label of Scharlemann cycle, which implies that all vertices of $\hat{\Gamma}_{a}^{+}$are labels of Scharlemann cycles, except the valence 4 vertex in a type (4) component. Since $\hat{\Gamma}_{a}^{+}$has at most two Scharlemann labels of each sign, we see that each $\hat{\Gamma}_{a}^{+}$is a union of two type (4) components. This will be ruled out in Lemma 5.10, completing the proof of the theorem.

Each vertex $u_{i}$ in $\Gamma_{a}$ has $\Delta$ edge endpoints labeled $j$. Define $\sigma\left(u_{i}, v_{j}\right)$ to be the number of those on positive edges minus the number of those on negative edges. In other words, it is the sum of the signs of the edges with an endpoint labeled $j$ at $u_{i}$.

Define a vertex $u$ of $\hat{\Gamma}_{a}^{+}$to be small if it is either of valence 2 or is a boundary vertex of valence 3. Note that a component of type (1) or (3) in Figure 4.2 has one small vertex, a component of type (10) has no small vertex, and all others have two small vertices.

Lemma 5.1 (1) $\sigma\left(u_{i}, v_{j}\right)=-\sigma\left(v_{j}, u_{i}\right)$.

(2) If $v_{j}$ is a small vertex in $\hat{\Gamma}_{b}^{+}$then $\sigma\left(u_{i}, v_{j}\right) \geq 0$ for all $i$.

(3) If $\hat{\Gamma}_{a}^{+}$has a boundary vertex $u_{i}$ of valence 2 , then $\sigma\left(u_{i}, v_{j}\right)<0$ for all but at most two $j$, at most one for each sign.

(4) If $\hat{\Gamma}_{a}^{+}$has a boundary vertex of valence 2 , then $\hat{\Gamma}_{b}^{+}$has at most one small vertex of each sign.

Proof. (1) This follows from the parity rule Lemma 2.2(1).

(2) If $v_{j}$ has valence 2 in $\hat{\Gamma}_{b}^{+}$then each label $i$ appears at most twice among the positive edge endpoints. If $v_{j}$ is a boundary vertex of valence 3 in $\hat{\Gamma}_{b}^{+}$then by Lemma $2.7(3)$ it is incident to at most $2 n_{a}$ adjacent positive edges in $\Gamma_{b}$, hence again each $i$ appears at most twice among the positive edge endpoints. Since $\Delta \geq 4$, the result follows.

(3) If $u_{i}$ is a boundary vertex of valence 2 then by Lemma 2.7(2) there are at most $n_{b}+2$ adjacent positive edges, so at most two labels appear more than once among the positive edge endpoints, and if there are two then they are adjacent, so there is only one for each sign.

(4) This follows immediately from (2) and (3).

Lemma 5.2 Suppose $\hat{\Gamma}_{a}^{+}$has a boundary vertex $u_{i}$ of valence 2. Then all components of $\hat{\Gamma}_{b}^{+}$are of type (1), (3) or (10). Moreover, for each sign there is at most one component with vertices of that sign which is of type (1) or (3). 
Proof. This follows immediately from Lemma 5.1(4) and the fact that a component of type (1) or (3) has one small vertex, a component of type (10) has no small vertex, and all others have two small vertices.

Lemma 5.3 Let $v_{j}$ be a vertex of a type (10) component $G$ of $\hat{\Gamma}_{b}^{+}$.

(1) $v_{j}$ is incident to at most $2 n_{a}+2$ positive edges in $\Gamma_{b}$.

(2) $\sigma\left(u_{i}, v_{j}\right) \geq 0$ for all but at most two $u_{i}$, one for each sign.

Proof. (1) By Lemma 2.7(2) the four families of adjacent parallel positive edges incident to $v_{j}$ contain $m \leq 2\left(n_{a}+2\right)$ edges. If $m>2 n_{a}+2$, then in particular one of the families contains more than $n_{a} / 2$ edges, so it contains a Scharlemann bigon. By Lemma 2.2(4) and Lemma 2.2(1) the labels at the endpoints of a loop at $v_{j}$ must have different parity, which rules out the possibility $m=2 n_{a}+3$. Hence $m=2 n_{a}+4$. Note that in this case there are at least 4 parallel loops $\left\{e_{1}, \ldots, e_{4}\right\}$, where $e_{1}$ is the outermost edge on the annulus containing $G$. By looking at the labels at the endpoints of these loops, we see that $e_{2}, e_{3}$ form a Scharlemann bigon, which contradicts Lemma 2.2(6) because $\left\{e_{1}, e_{4}\right\}$ is then an extended Scharlemann cycle.

(2) Since $v_{j}$ is a boundary vertex of $G$, the positive edges incident to $v_{j}$ are adjacent. Therefore (1) implies that $\sigma\left(v_{j}, u_{i}\right) \leq 0$ for all but at most two $i$, hence by Lemma 5.1(1) we have $\sigma\left(u_{i}, v_{j}\right) \geq 0$ for all but at most two $u_{i}$, and if there are two such $u_{i}$ then they are of opposite sign.

Lemma 5.4 $\hat{\Gamma}_{a}^{+}$cannot have two parallel boundary vertices of valence 2; in particular, no component $G$ of $\hat{\Gamma}_{a}^{+}$is of type (5), (6), (7) or (8).

Proof. Suppose to the contrary that $\hat{\Gamma}_{a}^{+}$has two boundary vertices $u_{i_{1}}, u_{i_{2}}$ of valence 2 , and of the same sign. By Lemma 5.2, each component $G^{\prime}$ of $\hat{\Gamma}_{b}^{+}$is of type (1), (3) or (10). If $G^{\prime}$ is of type (3) then it has a boundary vertex of valence 2 , so applying Lemma 5.2 to this vertex (with $\hat{\Gamma}_{a}^{+}$and $\hat{\Gamma}_{b}^{+}$switched), we see that $G$ must be of type (1), (3) or (10), which is a contradiction. Therefore $G^{\prime}$ must be of type (1) or (10).

By Lemma 5.1(3), $\sigma\left(u_{i_{1}}, v_{k}\right)<0$ for all but at most two $v_{k}$. Similarly for $\sigma\left(u_{i_{2}}, v_{k}\right)$. Since $n_{b}>4$, there is a vertex $v^{\prime}$ such that $\sigma\left(u_{r}, v^{\prime}\right)<0$ for both $r=i_{1}, i_{2}$. On the other hand, if $v^{\prime}$ is on a component $G^{\prime}$ and if $G^{\prime}$ is of type (1) then by Lemma 5.1(2) we have $\sigma\left(u_{r}, v^{\prime}\right)=-\sigma\left(v^{\prime}, u_{r}\right) \geq 0$ for all $u_{r}$, while if $G^{\prime}$ is of type (10) then Lemma 5.3(2) says $\sigma\left(u_{r}, v^{\prime}\right) \geq 0$ for either $r=i_{1}$ or $i_{2}$ because $u_{i_{1}}$ and $u_{i_{2}}$ are of the same sign. This is a contradiction.

Note that a vertex $u$ on a component $G$ of $\hat{\Gamma}_{a}^{+}$is a boundary vertex if it lies on one outermost essential cycle $C_{1}$ of $G$ but not the other one. In this case there is a unique component $G^{\prime}$ of $\hat{\Gamma}_{a}^{+}$and a unique outermost essential cycle $C_{2}$ on $G^{\prime}$ such that $C_{1} \cup C_{2}$ bounds an annulus on $\hat{F}_{a}$ whose interior contains no vertex of $\Gamma_{a}$. We say that $G^{\prime}$ and $C_{2}$ are adjacent to $u$.

Lemma 5.5 Let $u_{i}$ be a vertex on a type (10) component $G$ of $\hat{\Gamma}_{a}^{+}$. If $u_{i}$ is not a label of a Scharlemann cycle in $\Gamma_{b}$, then 
(i) the component $G^{\prime}$ of $\hat{\Gamma}_{a}^{+}$adjacent to $u_{i}$ is of type (1), (3) or (10);

(ii) $u_{i}$ is incident to exactly $2 n_{b}-2$ negative edges; and

(iii) $\hat{\Gamma}_{b}^{+}$has only two components, each of type (4) or (11).

Proof. We assume that $u_{i}$ is not a label of a Scharlemann cycle. Let $G^{\prime}$ and $C$ be the component and outermost cycle adjacent to $u_{i}$. If $C$ has a boundary vertex $u_{j}$ of valence at most 3 , then by Lemma $4.3 u_{j}$ is a label of a Scharlemann cycle. Since $u_{j}$ is a boundary vertex and there is no vertex between $C$ and the outermost cycle on $G$ containing $u_{i}$, the edges of the above Scharlemann cycle must connect $u_{j}$ to $u_{i}$, hence $u_{i}$ is also a label of the Scharlemann cycle, which is a contradiction. Also, if $G^{\prime}$ is of type (2) then by Lemma 4.1 each of its vertices is a label of a Scharlemann cycle. Recall that the edges of a Scharlemann cycle in $\Gamma_{b}$ cannot lie in a disk on $\hat{F}_{a}$, hence the edges of one of the Scharlemann cycles must connect a vertex on $C$ to $u_{i}$, which again is a contradiction. Therefore $C$ does not have a boundary vertex of valence at most 3 , and it is not on a type (2) component. Examining the graphs in Figure 4.2, we see that $G^{\prime}$ must be of type (1), (3) or (10). Moreover, if it is of type (3) then $C$ is the loop there. In any case, $C$ contains only one vertex.

Let $t$ be the number of negative edges incident to $u_{i}$. Since $C$ has only one vertex $u_{j}, u_{i}$ is incident to at most two families of negative edges $\hat{e}_{1}, \hat{e}_{2}$, all connecting $u_{i}$ to $u_{j}$, so by Lemma $2.7(1) t \leq 2 n_{b}$. On the other hand, by Lemma $5.3 u_{i}$ is incident to at most $2 n_{b}+2$ positive edges, so $t \geq 2 n_{b}-2$. Therefore we have $2 n_{b} \geq t \geq 2 n_{b}-2$.

First assume $t=2 n_{b}$. Then each of $\hat{e}_{1}$ and $\hat{e}_{2}$ contains exactly $n_{b}$ edges. Since $u_{i}$ is not a label of Scharlemann cycle, by Lemma 2.4 these $2 n_{b}$ edges are mutually non-parallel on $\Gamma_{b}$, hence $\hat{\Gamma}_{b}^{+}$has at least $2 n_{b}$ edges. On the other hand, by Lemma 4.1 it cannot have more than $2 n_{b}$ such edges, hence $\hat{\Gamma}_{b}^{+}$has exactly $2 n_{b}$ edges, each containing exactly one edge in $\hat{e}_{1} \cup \hat{e}_{2}$. Counting the number of edges on each graph in Figure 4.2, we see that each component of $\hat{\Gamma}_{b}^{+}$ must be of type (10) or (11). Also, a component of type (11) has a vertex $v_{k}$ of valence 5 in $\hat{\Gamma}_{b}^{+}$, so the above implies that the label $k$ appears 5 times among the endpoints of edges in $\hat{e}_{1} \cup \hat{e}_{2}$, which is absurd. This rules out the possibility for a component to be of type (11). Now notice that these two families of $n_{b}$ parallel edges have the same transition function, hence if some edge has the same labels on its two endpoints, then they all do. It follows that no component can be of type (10) because it has both loop and non-loop edges. This completes the proof for the case $t=2 n_{b}$.

If $t=2 n_{b}-1$ then one of $\hat{e}_{1}, \hat{e}_{2}$ contains $n_{b}$ edges and the other contains $n_{b}-1$ edges. Examining the labels at the endpoints of these edges we see that if an edge in $\hat{e}_{1}$ has labels of the same parity at its two endpoints then an edges in $\hat{e}_{2}$ would have labels of different parities at its endpoints, and vice versa. This contradicts the parity rule (Lemma 2.2(1)).

We can now assume $t=2 n_{b}-2$. Without loss of generality we may assume that the labels of the endpoints of $\hat{e}_{1} \cup \hat{e}_{2}$ appear as $1,2, \ldots, n_{b}, 1, \ldots, n_{b}-2$ on $\partial u_{i}$ when traveling clockwise, and we assume that the first $n_{b}$ are endpoints of $\hat{e}_{1}$. (The other cases are similar.) Let $e_{p}^{k}(k=1,2)$ be the edge in $\hat{e}_{k}$ with label 
$p$ at $u_{i}$, and assume that the label of $e_{1}^{1}$ on $u_{j}$ is $1+r$ for some $r$. Then one can check that the label of $e_{p}^{1}$ on $u_{j}$ is $p+r$, and the label of $e_{p}^{2}$ on $u_{j}$ is $p+r+2$. (All labels are integers $\bmod n_{b}$.) Hence for any $p$ between 3 and $n_{b}$, the edges $e_{p}^{1}$ and $e_{p-2}^{2}$ have the same label $p+r$ at $u_{j}$. On $\Gamma_{b}$ this implies that there are two positive edges, connecting $v_{p}$ to $v_{p+r}$ and $v_{p+r}$ to $v_{p-2}$, so $v_{p}$ are $v_{p-2}$ are in the same component of $\hat{\Gamma}_{b}^{+}$. Since this is true for all $p$ between 3 and $n_{b}$, it follows that $\hat{\Gamma}_{b}^{+}$has only two components.

By Lemmas 2.8 and 2.2(4) $\hat{\Gamma}_{b}^{+}$has the same number of positive vertices and negative vertices, hence each component $G$ has at least three vertices. This rules out the possibility for $G$ to be of type (1), (2), (3), (9) or (10). Combined with Lemme 5.4 we see that each component of $\hat{\Gamma}_{b}^{+}$is of type (4) or (11).

Lemma 5.6 No component of $\hat{\Gamma}_{a}^{+}$is of type (3).

Proof. By Lemma 5.2 if $\hat{\Gamma}_{a}^{+}$has a component of type (3) then each component of $\hat{\Gamma}_{b}^{+}$is of type (1), (3) or (10), and there is at most one component of type (1) or (3) for each sign. Since $n_{b}>4$ and a component of type (1) or (3) has at most 2 vertices, there is at least one component $G$ of $\hat{\Gamma}_{b}^{+}$of type (10) and at least one other component $G^{\prime}$ of the same sign. On the other hand, by Lemma 5.5 each vertex of $G$ is a label of a Scharlemann cycle, and by Lemmas 4.1 and 4.3 at least one vertex of $G^{\prime}$ is a label of a Scharlemann cycle, so there are at least three labels of Scharlemann cycles of the same sign, contradicting Lemma $2.3(4)$.

Lemma 5.7 No component of $\hat{\Gamma}_{a}^{+}$is of type (11).

Proof. An outermost cycle on a component $G$ of type (11) contains two parallel vertices $u_{i}$ and $u_{j}$, where $u_{i}$ is of valence 3 and hence the label of a Scharlemann bigon (Lemma 4.3 ), and $u_{j}$ has valence 5 . If $\left\{e_{1}, e_{2}\right\}$ is a Scharlemann bigon on $\Gamma_{b}$ with label pair $\{i, i+1\}$, say, then on $\Gamma_{a}$ these edges form an essential curve containing the vertices $u_{i}$ and $u_{i+1}$, which separates $u_{j}$ from all other vertices of opposite sign, hence all negative edges incident to $u_{j}$ have their other endpoints on $u_{i+1}$, and they are all parallel. Thus $u_{j}$ has at most $n_{b}$ negative edges, and hence at least $3 n_{b}$ adjacent positive edges. In particular, each label appears at least three times among endpoints of positive edges at $u_{j}$. Dually, each vertex $v_{k}$ in $\Gamma_{b}$ is incident to at least three negative edges labeled $j$ at $v_{k}$. If $v_{k}$ is a boundary vertex, then this implies that it is incident to at least $2 n_{a}+1$ negative edges, so by Lemma 4.1 it is a label of a Scharlemann cycle.

By Lemmas 5.4 and 5.6 a component of $\hat{\Gamma}_{b}^{+}$is of type (1), (2), (4), (9), (10) or (11). By the above and Lemma 4.1 all vertices of $\Gamma_{b}$ except those with valence 4 in type (4) components are labels of Scharlemann cycles. Since $n_{b}>4$ and there are at most two Scharlemann labels for each sign, we see that $\hat{\Gamma}_{b}^{+}$has only two components, each of type $(4)$, so $n_{b}=6$, and $\hat{\Gamma}_{b}^{+}$has 10 positive edges. By Lemma $2.5 \hat{\Gamma}_{b}$ has at most $3 n_{b}-10=8$ negative edges. On the other hand, we have shown that $u_{j}$ in $\Gamma_{a}$ is incident to at least $3 n_{b}=18$ positive edges; since 
no three of them are parallel in $\Gamma_{b}, \hat{\Gamma}_{b}$ has at least $18 / 2=9$ negative edges, which is a contradiction.

Lemma 5.8 Each vertex of a type (10) component of $\hat{\Gamma}_{a}^{+}$is a label of a Scharlemann bigon.

Proof. Suppose that a vertex $u_{i}$ of a type (10) component of $\hat{\Gamma}_{a}^{+}$is not a label of a Scharlemann bigon. By Lemmas 5.5 and $5.7 \hat{\Gamma}_{b}^{+}$is a union of two type (4) components, so $n_{b}=6, \hat{\Gamma}_{b}$ has 10 positive edges, and no more than $3 n_{b}-10=8$ negative edges.

By Lemma 5.5(ii) $u_{i}$ is incident to $(\Delta-2) n_{b}+2=6 \Delta-10$ positive edges (loops counted twice). By Lemma 2.7(1) no three of these are parallel in $\Gamma_{b}$, hence they represent at least $3 \Delta-5$ negative edges in $\hat{\Gamma}_{b}$. Therefore $\Delta=4$, and we have at least 7 negative edges in $\hat{\Gamma}_{b}$. We need to find two more to get a contradiction.

By Lemma 5.5(i) and Lemma 5.6 the component $G$ of $\hat{\Gamma}_{a}^{+}$adjacent to $u_{i}$ is of type (1) or (10), so the outermost cycle of $G$ adjacent to $u_{i}$ has a single vertex $u_{j}$ and a single edge $E_{0}$. We claim that $E_{0}$ contains at least two edges of $\Gamma_{b}$.

If $G$ is of type (10), then $u_{j}$ is incident to four families of positive edges in $\Gamma_{a}$, with a total of $2 n_{b}+2=14$ edges, where loops are counted twice. By Lemma 2.3(3) each family contains no more than 4 edges, so the loop edge $E_{0}$ contains at least $(14-2 \times 4) / 2=3$ edges of $\Gamma_{b}$. If $G$ is of type (1) then since no three negative edges incident to $u_{j}$ are parallel in $\Gamma_{b}$, and since $\hat{\Gamma}_{b}^{+}$has only 10 edges, we see that $u_{j}$ is incident to at most 20 negative edges, hence $E_{0}$ contains at least $(24-20) / 2=2$ edges. This completes the proof of the above claim.

Let $e_{1}^{\prime}, e_{2}^{\prime}$ be the two edges in $E_{0}$ closest to $u_{i}$. By Lemma 2.2(2) they are not parallel on $\Gamma_{b}$. We claim that on $\Gamma_{b}$ neither of them is parallel to any edge incident to $u_{i}$, hence $\hat{\Gamma}_{b}$ contains at least $7+2=9$ negative edges. This will be a contradiction as we have shown above that $\hat{\Gamma}_{b}$ has at most 8 negative edges.

By Lemma 5.5(ii) there are exactly $2 n_{b}-2=10$ negative edges $e_{1}, \ldots, e_{10}$ connecting $u_{i}$ to $u_{j}$. Without loss of generality we may assume that the sequence of labels of the endpoints of these edges at $u_{i}$ is $1, \ldots, 6,1, \ldots, 4$, counting clockwise, and the labels of their endpoints at $u_{j}$ are $r+2, r+3, \ldots, r-1$, counting counterclockwise. Thus $\left\{e_{1}^{\prime}, e_{2}^{\prime}\right\}$ is a Scharlemann bigon with label pair $\{r, r+1\}$.

Since $e_{i}^{\prime}$ is a loop, by Lemma 2.3(5) if it is parallel in $\Gamma_{b}$ to an edge $e$ incident to $u_{i}$ then $e$ is also a loop. Note that $e_{i}^{\prime}$ and $e$ must have the same label pair. Let $E_{3}$ be the loop of $\hat{\Gamma}_{a}$ based at $u_{i}$. It has at most four edges $e_{1}^{\prime \prime}, e_{2}^{\prime \prime}, e_{3}^{\prime \prime}, e_{4}^{\prime \prime}$, with label pairs $\{5,6\},\{6,5\},\{1,4\},\{2,3\}$, respectively. By Lemma 2.3(2) we have $\{r, r+1\} \neq\{2,3\}$, hence if $e_{i}^{\prime}$ is parallel to some $e_{j}^{\prime \prime}$ then $\{r, r+1\}=\{5,6\}$, so $r=5$, and hence the label sequence of the above negative edges at $u_{j}$ is also $1, \ldots, 6,1, \ldots, 4$.

The 10 edges $e_{1}, \ldots, e_{10}$ are divided into two families $E_{1}, E_{2}$. Since $\left|E_{i}\right| \leq 6$, we have $\left|E_{1}\right|=4,5$, or 6 . If $\left|E_{1}\right|=5$ then the edge $e_{1}$ would have label 1 at $u_{i}$ and label 6 at $u_{j}$. Since $v_{1}$ and $v_{6}$ on $\Gamma_{b}$ are antiparallel, this is impossible by the parity rule. If $\left|E_{1}\right|=4$ then $e_{1}$ has the same label 1 at its two endpoints, which contradicts the fact that $\Gamma_{b}$ has no loop. Similarly if $\left|E_{1}\right|=6$ then $e_{7}$ 
has the same label 1 at its two endpoints, which is again a contradiction. This completes the proof of the Lemma.

Lemma 5.9 Each $\hat{\Gamma}_{a}^{+}$is a union of two type (4) components.

Proof. By Lemmas 5.4, 5.6 and 5.7, each component $G$ of $\hat{\Gamma}_{a}^{+}$is of type (1), (2), (4), (9) or (10). By Lemmas 4.1, 4.3 and 5.8, we see that all vertices $u_{i}$ of $G$ are labels of Scharlemann bigons, unless $G$ is of type (4) and $u_{i}$ is the vertex of valence 4 in $G$. Since $n_{a}>4$ and $\hat{\Gamma}_{a}^{+}$has at most two vertices which are labels of Scharlemann bigons for each sign, we see that $\hat{\Gamma}_{a}^{+}$consists of exactly two components, each of type (4).

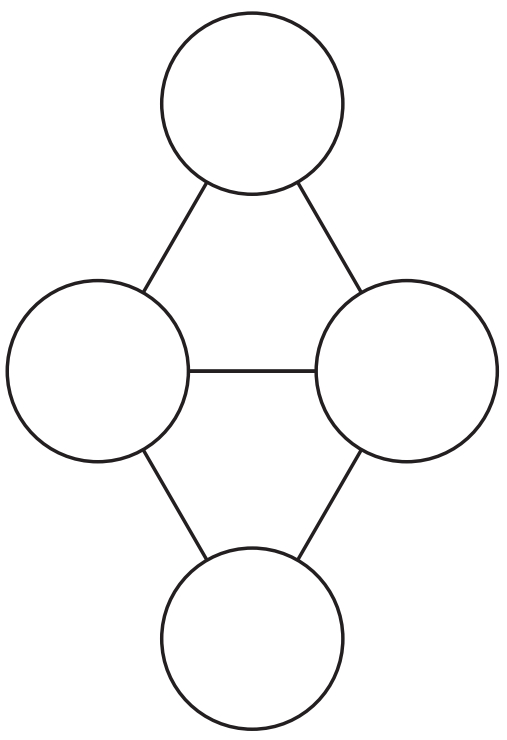

(a)

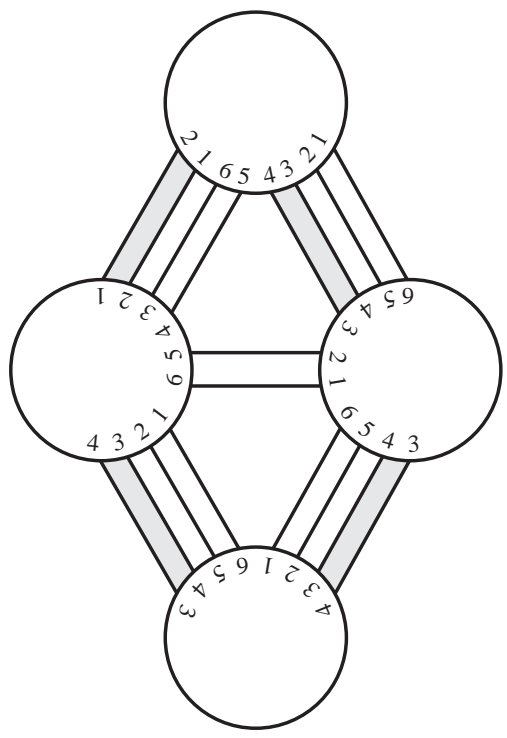

(b)

Figure 5.1

Lemma 5.10 One of the $\hat{\Gamma}_{a}^{+}$is not a union of two type (4) components.

Proof. Assume that each $\hat{\Gamma}_{a}^{+}$is a union of two type (4) components. Each vertex of $\Gamma_{a}$ has valence $\Delta n_{b} \geq 24$, hence $\Gamma_{a}$ has at least 72 edges. Since a positive edge in $\Gamma_{a}$ is a negative edge in $\Gamma_{b}$, we may assume that $\Gamma_{1}$ has no more negative edges than positive edges, so $\Gamma_{1}^{+}$has at least 36 positive edges. Thus one component $G$ of $\Gamma_{1}^{+}$has at least 18 edges. Denote by $\hat{G}$ the reduced 
graph of $G$. It is of type (4), so it is obtained from the graph in Figure 5.1(a) by identifying the top and bottom vertices.

Let $E_{1}, \ldots, E_{5}$ be the edges of $\hat{G}$. Denote by $\left|E_{i}\right|$ the number of edges of $G$ in $E_{i}$, and call it the weight of $E_{i}$. By Lemma 2.3(3), each $\left|E_{i}\right| \leq 4$. Since $G$ has at least 18 edges, up to relabeling the weights of the edges are at least $(4,4,4,4,2)$ or $(4,4,4,3,3)$.

Let $D$ be a triangle face of $\hat{G}$, and let $E_{1}, E_{2}, E_{3}$ be the edges of $D$. We will also use $D$ to denote the corresponding triangle face in $G$. If $\left|E_{i}\right|=4$ then by Lemma $2.4 E_{i}$ contains a Scharlemann bigon, which must be at one end of the family of parallel edges in $E_{i}$. We say that the Scharlemann bigon in $E_{i}$ is adjacent to $D$ if one of its edges is on the boundary of $D$.

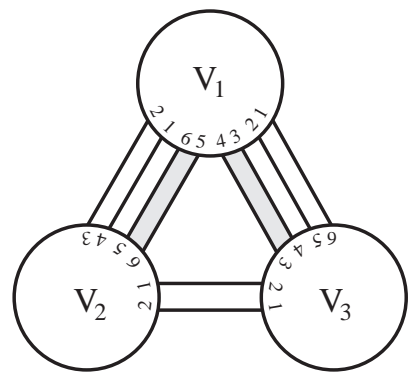

(a)

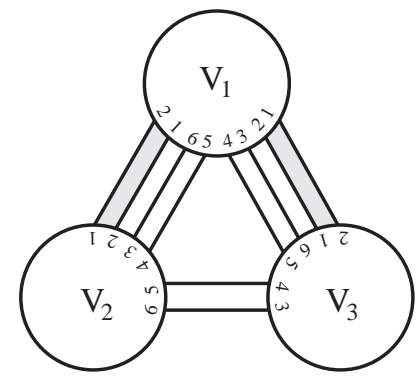

(b)

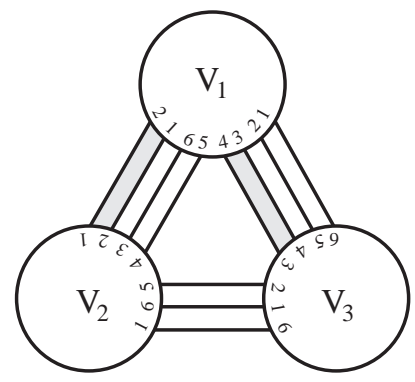

(c)

Figure 5.2

Sublemma If $\left|E_{1}\right|=\left|E_{2}\right|=4$, then (i) $\left|E_{3}\right|=2$, and (ii) exactly one of $E_{1}$ and $E_{2}$ has its Scharlemann bigon adjacent to $D$.

Proof. Let $V_{1}$ be the fat vertex incident to both $E_{1}$ and $E_{2}$. Without loss of generality we may assume that the labels on $\partial V_{1}$ are as shown in Figure 5.2(a), where $E_{1}$ is the upper right family of edges. Note that the positions of the Scharlemann bigons in $E_{1}, E_{2}$ determine the labels on $\partial V_{2}$ and $\partial V_{3}$.

If both $E_{1}$ and $E_{2}$ have their Scharlemann bigons adjacent to $D$, then the labels are as shown in Figure 5.2(a), in which case we have three Scharlemann bigons with disjoint label pairs, contradicting Lemma 2.3(4). If both Scharlemann bigons of $E_{1}, E_{2}$ are non-adjacent to $D$, then the labels are as shown in Figure 5.2(b), in which case the edges adjacent to those of $D$ form an extended Scharlemann cycle, which contradicts Lemma 2.2(6). This proves (ii).

We may now assume without loss of generality that the Scharlemann bigon of $E_{1}$ is adjacent to $D$ while that of $E_{2}$ is not adjacent to $D$. See Figure 5.2(c). In this case the label pair of the Scharlemann bigon in $E_{1}$ is $\{3,4\}$. If $\left|E_{3}\right| \geq 3$ then $E_{3}$ contains a Scharlemann bigon with label pair $\{6,1\}$. This contradicts Lemma 2.3(2), completing the proof of the sublemma. 
If the weights of the $E_{i}$ are $(4,4,4,3,3)$, or if the weights are $(4,4,4,4,2)$ and the horizontal edge in Figure 5.1(a) has weight 4, then the boundary edges of one of the triangles in Figure 5.1(a) have weights $(4,4,3)$ or $(4,4,4)$, which contradicts the sublemma. Therefore the edges of $G$ are exactly as shown in Figure 5.1(b). As in the proof of the sublemma, we may assume that the labels at the three vertices in the upper triangle of $G$ are as shown in Figure 5.1(b). The Scharlemann bigons in the upper triangle have label pairs $\{3,4\}$ and $\{1,2\}$, hence by Lemma 2.3(4) $G$ cannot have a Scharlemann bigon on label pair $\{5,6\}$. Therefore the labels of the endpoints of the lower-right edges must be as shown in Figure 5.1(b). This determines the labels at the lower vertex. But then neither Scharlemann bigon in the lower triangle is adjacent to the triangle, contradicting the sublemma.

Proposition 5.11 The case that both $n_{1}, n_{2}>4$ is impossible.

Proof. This follows from the contradiction between Lemma 5.9 and Lemma 5.10 .

\section{$6 \quad$ Kleinian graphs}

In Sections $6-11$ we will improve Proposition 5.11 to show that $n_{i} \leq 2$ for $i=1$ or 2 . For the most part we will assume that $n_{a}=4$. In this section we prove some useful lemmas. In particular, Lemmas $6.2-6.5$ study kleinian graphs. Lemma 6.2 gives basic properties of kleinian graphs, which will also be used later in studying the case $n_{a}=2$.

Definition 6.1 The graph $\Gamma_{a}$ is said to be kleinian if $\hat{F}_{a}$ bounds a twisted Ibundle over the Klein bottle $N(K)$ such that each component of $N(K) \cap V_{a}$ is a $D^{2} \times I$, and each component of $N(K) \cap F_{b}$ is a bigon.

By Lemma 2.12, if $M\left(r_{a}\right)$ contains a Klein bottle $K$ intersecting $K_{a}$ at $n_{a} / 2$ points then $\partial N(K)$ is an essential torus intersecting $K_{a}$ at $n_{a}$ points, hence in this case we may assume that $\hat{F}_{a}=\partial N(K)$, where $N(K)$ is a small regular neighborhood of $K$; in particular, $\Gamma_{a}$ is kleinian. In this case $N(K)$ is called the black region, and all faces of $\Gamma_{b}$ lying in this region are called black faces, and the others white faces. We assume that the vertices of $\Gamma_{a}$ have been labeled so that $u_{2 i-1} \cup u_{2 i}$ lie on the same component of $V_{a} \cap N(K)$. The following lemma lists the main properties of kleinian graphs.

Lemma 6.2 Suppose $\Gamma_{a}$ is kleinian. Then

(1) each black face of $\Gamma_{b}$ is a bigon;

(2) each family of parallel edges in $\Gamma_{b}$ contains an even number of edges;

(3) $\Gamma_{b}$ has no white Scharlemann disk, hence any Scharlemann cycle of $\Gamma_{b}$ has label pair $\{k, k+1\}$ with $k$ odd;

(4) there is a free involution of $\hat{F}_{a}$, which preserves $\Gamma_{a}$, sending $u_{2 i-1}$ to $u_{2 i}$ and preserving the labels of edge endpoints. 
Proof. (1) follows from the definition. (2) follows from (1) because if there is a family containing an odd number of edges then one side of that family would be adjacent to a black face, which is not a bigon.

(3) Each edge of a white face is adjacent to a black bigon, so if there is a white Scharlemann disk then the edges of the Scharlemann cycle and the adjacent edges would form an extended Scharlemann cycle, which would be a contradiction to Lemma 2.2(6).

(4) We may assume that the Dehn filling solid torus $V_{a}$ and the surface $F_{b}$ intersect $N(K)$ in $I$-fibers. Thus the involution of $\hat{F}_{a}$ obtained by mapping each point to the other end of the $I$-fiber gives rise to the required involution of $\Gamma_{a}$.

Lemma 6.3 Suppose $n_{a}=4$. Then $\Gamma_{a}$ is kleinian if each vertex of $\Gamma_{a}$ is a label of a Scharlemann bigon in $\Gamma_{b}$.

Proof. Without loss of generality we may assume that $\Gamma_{b}$ has a (12) Scharlemann bigon. By assumption there is a Scharlemann bigon with 3 as a label. If there is no (34) Scharlemann bigon then this Scharlemann bigon must have label pair (23). Similarly the Scharlemann bigon with 4 as a label must have label pair (14). We may therefore relabel the vertices of $\Gamma_{a}$ so that the label pairs of the above Scharlemann bigons are (12) and (34) respectively.

Shrinking the Dehn filling solid torus to its core, the Scharlemann bigons become Möbius bands $B_{12}$ and $B_{34}$ in $M\left(r_{a}\right)$. The union of these Möbius bands, together with an annulus on $\hat{F}_{a}$, becomes a Klein bottle which can be perturbed to intersect the core of the Dehn filling solid torus at $2=n_{a} / 2$ points. By the convention after Definition 6.1, $\hat{F}_{a}$ should have been chosen so that $\Gamma_{a}$ is kleinian.

Lemma 6.4 Suppose $n_{a}=4$. Then $\Gamma_{a}$ is kleinian if one of the following holds.

(1) $\Gamma_{b}$ has a family of 4 parallel positive edges.

(2) $\Gamma_{b}$ is positive.

(3) $\hat{\Gamma}_{b}^{+}$has a full vertex $v_{j}$ of valence at most $\%$.

(4) $\hat{\Gamma}_{b}^{+}$contains 4 adjacent families of positive edges with a total of at least 12 edges.

Proof. (1) Each label appears exactly twice among the edge endpoints of a family of four parallel positive edges, hence by Lemma 2.4 it is a label of a Scharlemann bigon.

(2) If $\Gamma_{b}$ is positive then every vertex $u_{i}$ of $\Gamma_{a}$ is incident to at least $4 n_{b}$ negative edges, two of which must be parallel in $\Gamma_{b}$ because by Lemma 2.5 $\hat{\Gamma}_{b}$ contains at most $3 n_{b}$ edges. Hence by Lemma 2.4 these two edges form a Scharlemann bigon with $i$ as a label. Since this is true for all $i, \Gamma_{a}$ is kleinian by Lemma 6.3 .

(3) Consider the subgraph $G$ of $\hat{\Gamma}_{a}$ consisting of negative edges. Then the signs of the vertices around the boundary of a face of $G$ alternate, hence each face has an even number of edges. Using an Euler characteristic argument one 
can show that $G$ contains at most $2 n_{a}=8$ edges. By (2) we may assume $\Gamma_{b}$ is not positive, so by Lemma 2.3(1) no $3 j$-edges are parallel on $\Gamma_{a}$, hence $G$ has exactly 8 negative edges, each containing exactly $2 j$-edges, with one $j$ label at each ending vertex. Since each vertex $u_{i}$ has $4 j$-labels, we see that $u_{i}$ is incident to exactly $8 j$-edges, two of which must be parallel in $\Gamma_{b}$ because $\operatorname{val}\left(v_{j}, \hat{\Gamma}_{b}^{+}\right) \leq 7$. By Lemma 2.4 they form a Scharlemann bigon with $i$ as one of its labels.

(4) By (1) we may assume that each family contains exactly 3 edges, so the labels at the endpoints of the middle edge in each family are the labels of a Scharlemann bigon. It is easy to see that the 4 endpoints of the middle edges at the vertex are mutually distinct, hence include all labels.
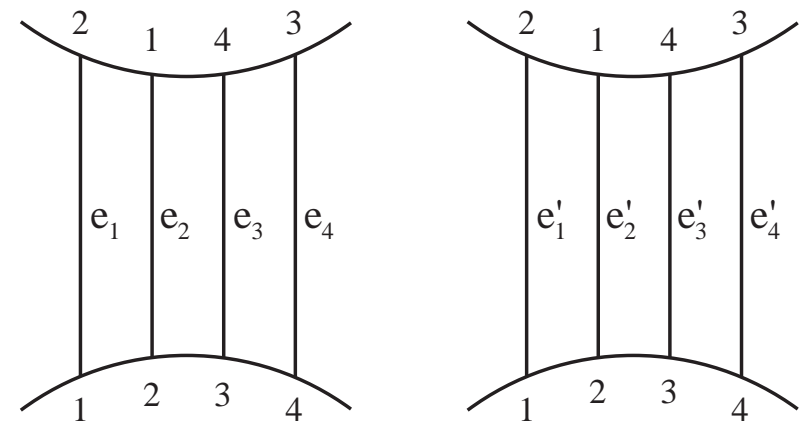

Figure 6.1

Lemma 6.5 Suppose $n_{a}=4$. Let $e_{1} \cup e_{2} \cup e_{3} \cup e_{4}$ and $e_{1}^{\prime} \cup e_{2}^{\prime} \cup e_{3}^{\prime} \cup e_{4}^{\prime}$ be two families of parallel edges in $\Gamma_{b}$ as shown in Figure 6.1. Then $e_{i}$ is parallel to $e_{i}^{\prime}$ on $\Gamma_{a}$ for all $i$.

Proof. Since $e_{1} \cup e_{2}$ and $e_{3} \cup e_{4}$ form two disjoint essential cycles on $\hat{F}_{a}$ by Lemma 2.2(5), any (12)-edge must be parallel to $e_{1}$ or $e_{2}$ and any (34)-edge parallel to $e_{3}$ or $e_{4}$ on $\Gamma_{a}$. Note also that if $e_{1}$ is parallel to $e_{2}^{\prime}$ on $\Gamma_{a}$ then $e_{2}$ must be parallel to $e_{1}^{\prime}$ (instead of $e_{2}^{\prime}$ ) on $\Gamma_{a}$ as otherwise $e_{1}, e_{2}$ would be parallel on both graphs. Therefore if the result is not true then either $e_{1}$ is parallel to $e_{2}^{\prime}$ or $e_{4}$ is parallel to $e_{3}^{\prime}$, so there is a subset $e_{r}^{\prime} \cup \ldots \cup e_{s}^{\prime}$ of the second family containing less than 4 edges, such that $e_{r}^{\prime} \cup e_{1}$ and $e_{s}^{\prime} \cup e_{4}$ are parallel pairs on $\Gamma_{a}$. This contradicts Lemma 2.19

Lemma 6.6 Suppose $n_{a}=4$ and $\Gamma_{b}$ is non-positive.

(1) No vertex $v_{j}$ of $\Gamma_{b}$ can have two families of 4 positive edges with the same label sequence on $\partial v_{j}$. In particular, $v_{j}$ cannot have two adjacent families of 4 positive edges.

(2) If $\Gamma_{a}$ is kleinian, then two adjacent families of positive edges of $\Gamma_{b}$ contain at most 6 edges, three contain at most 10, and four contain at most 12.

(3) A full vertex of $\hat{\Gamma}_{b}^{+}$has valence at least 6 . 
Proof. (1) If there are two families of 4 positive edges with the same label sequence on $\partial v_{j}$ then by Lemma 6.5 the two starting edges $e_{1}, e_{1}^{\prime}$ of these families will be parallel in $\Gamma_{a}$. If $e_{1}, e_{1}^{\prime}$ have label $i$ at $v_{j}$ then on $\Gamma_{a}$ they have the same label $j$ at $u_{i}$, so there are $n_{b}+1$ parallel negative edges at $u_{i}$, and hence by Lemma 2.3(1) $\Gamma_{b}$ would be positive, a contradiction.

(2) By Lemma 6.2(2) the number of edges in each family of positive edges is either 2 or 4, so by (1) two adjacent families contain a total of at most 6 edges. The other two cases follow from this.

(3) Otherwise by Lemma 6.4(3) $\Gamma_{b}$ is kleinian, so the weight of each positive family of $\Gamma_{b}$ is either 2 or 4 . If some full vertex $v_{i}$ has valence 5 or less in $\hat{\Gamma}_{b}^{+}$ then it has two adjacent edge of weight 4 , contradicting (1).

A bigon is called a non-Scharlemann bigon if it is not a Scharlemann bigon.

Lemma 6.7 Suppose $n_{a}=4$ and $\Gamma_{a}$ is kleinian.

(1) Exactly one edge on the boundary of a triangle face of $\hat{\Gamma}_{b}^{+}$represents a non-Scharlemann bigon. Each of the other two represents either a Scharlemann bigon or a union of two Scharlemann bigons.

(2) If some vertex $v_{i}$ is incident to two edges of weight 4 in $\hat{\Gamma}_{b}^{+}$then any other edge of $\hat{\Gamma}_{b}^{+}$incident to $v_{i}$ represents a non-Scharlemann bigon.

Proof. (1) Let $\hat{e}_{1}, \hat{e}_{2}, \hat{e}_{3}$ be the edges of a triangle face $\delta$ of $\hat{\Gamma}_{b}^{+}$. By Lemma 6.2(2) each edge of $\hat{\Gamma}_{b}^{+}$represents 2 or 4 edges. From the labeling of the edges around $\delta$ one can see that there are exactly one or three $\hat{e}_{i}$ which are neither a Scharlemann bigon nor a union of two Scharlemann bigons. If there are three then they form an extended Scharlemann cycle, which is impossible by Lemma 2.2(6). Hence there must be exactly one such $\hat{e}_{i}$.

(2) Otherwise $v_{i}$ would be incident to 5 Scharlemann bigons, three of which have the same label pair, say $\{1,2\}$. Then on $\Gamma_{a}$ there are six $i$-edges connecting $u_{1}$ to $u_{2}$, which form at most two families because there is a Scharlemann cocycle containing $u_{3}, u_{4}$. It follows there there are three $i$ labels at the endpoints of a family, so it contains more than $n_{b}$ edges, contradicting Lemma 2.3(1).

Suppose $\Delta=4$. Then a label $j$ is a jumping label at $u_{i}$ if the signs of the four $j$-edges incident to $u_{i}$ alternate.

Lemma 6.8 Suppose $\Delta=4$. Then a label $i$ is a jumping label at $v_{j}$ if and only if $j$ is a jumping label at $u_{i}$. In particular, if $v_{j}$ is a boundary vertex of $\hat{\Gamma}_{b}^{+}$then $j$ is not a jumping label at any $u_{i}$.

Proof. This follows from the Jumping Lemma 2.18. Let $x_{1}, \ldots, x_{4}$ be the four points of $u_{i} \cap v_{j}$. Since $\Delta=4$, the jumping number must be \pm 1 . Therefore they appear in this order on both $\partial u_{i}$ and $\partial v_{j}$, appropriately oriented. If $j$ is a jumping label at $u_{i}$ then we may assume $x_{1}, x_{3}$ are positive edge endpoints and $x_{2}, x_{4}$ are negative edge endpoints on $\partial u_{i}$, which by the parity rule implies that $x_{1}, x_{3}$ are negative edge endpoints and $x_{2}, x_{4}$ are positive edge endpoints on $\partial v_{j}$, hence $i$ is a jumping label at $v_{j}$. 
Lemma 6.9 Suppose $n_{a}=4, n_{b} \geq 4$, and $\Gamma_{a}$ is non-positive. Then $\hat{F}_{a}$ is separating. In particular, $u_{1}$ is parallel to $u_{3}$ and antiparallel to $u_{2}$ and $u_{4}$.

Proof. The result follows from Lemmas 2.8 and 2.2(4) if some vertex $u_{i}$ is incident to more than $n_{b}$ negative edges. In particular, since each family of positive edges contain no more than $n_{b}$ edges, the result is true if $\operatorname{val}\left(u_{i}, \hat{\Gamma}_{a}^{+}\right) \leq 2$ for some $i$. Hence we may assume that $\operatorname{val}\left(u_{i}, \hat{\Gamma}_{a}^{+}\right)>2$ for all $i$. One can check that in this case each component of $\hat{\Gamma}_{a}^{+}$must be as shown in Figure 4.2(9), (10) or (11). In each case $\hat{\Gamma}_{a}^{+}$has a boundary vertex $u_{i}$ of valence at most 4 , so if $n_{b}>4$ then by Lemma 2.7(b) the 4 families of positive edges contain at most $2\left(n_{b}+2\right)<3 n_{b}$ edges, hence $u_{i}$ is incident to more than $n_{b}$ negative edges and the result follows. Similarly if $\hat{\Gamma}_{a}^{+}$has a boundary vertex of valence at most 3 then by Lemma 2.7(c) it is incident to at most $2 n_{b}$ positive edges in $\Gamma_{a}$ and the result follows. Therefore we may assume $n_{b}=4$.

A vertex $u_{i}$ on a component in Figure $4.2(9)$ or (10) is a boundary vertex of valence 3 or 4 in $\hat{\Gamma}_{a}^{+}$, so by Lemma $2.7(\mathrm{~b})-(\mathrm{c})$ it is incident to less than $3 n_{b}$ positive edges, and hence more than $n_{b}$ negative edges, unless $n_{b}=4$ and $\operatorname{val}\left(u_{i}, \hat{\Gamma}_{a}^{+}\right)=4$. In particular $\hat{\Gamma}_{a}^{+}$must be of type (10) in Figure 4.2. In this last case by Lemma 6.4(4) $\Gamma_{b}$ is kleinian, so by Lemma 6.2(2) each family of positive edges of $\Gamma_{a}$ contains either 2 or 4 edges. Since there is a total of at least 12 edges and by Lemma 6.6(2) two adjacent families contain at most 6 edges, the weights of the four edges of $\hat{\Gamma}_{a}^{+}$incident to $u_{i}$ must be $(4,2,4,2)$ successively. However since the first and the last belong to a loop in $\hat{\Gamma}_{a}^{+}$, their weights must be the same, which is a contradiction.

\section{If $n_{a}=4, n_{b} \geq 4$ and $\hat{\Gamma}_{a}^{+}$has a small component then $\Gamma_{a}$ is kleinian.}

A component of $\hat{\Gamma}_{a}^{+}$is small if it has at most two edges; otherwise it is large. In this section we will show that if $n_{a}=4, n_{b} \geq 4$ and $\hat{\Gamma}_{a}^{+}$has a small component then $\Gamma_{a}$ is kleinian. It is easy to see that the assumption implies that either $\operatorname{val}\left(u_{1}, \hat{\Gamma}_{a}^{+}\right) \leq 1, \operatorname{or} \operatorname{val}\left(u_{1}, \hat{\Gamma}_{a}^{+}\right)=\operatorname{val}\left(u_{3}, \hat{\Gamma}_{a}^{+}\right)=2$ up to relabeling. (See the proof of Proposition 7.6.) The two cases are handled in Lemmas 7.3 and 7.5, respectively.

Lemma 7.1 Suppose $\Gamma_{a}$ contains a loop edge at $u_{3}$. Then $\Gamma_{b}$ cannot contain both (12)- and (14)-Scharlemann bigons.

Proof. The loop $e$ at $u_{3}$ must be essential, otherwise it would bound some disk containing some vertex and hence one of the Scharlemann cocycles in its interior, which contradicts Lemma 2.2(5). Now the (12)- and (14)-Scharlemann bigons in $\Gamma_{b}$ form two essential cycles in $\Gamma_{a}$ disjoint from $e$, so they must be isotopic on $\hat{F}_{a}$, bounding a disk face containing no vertices of $\Gamma_{a}$ in its interior. This is a contradiction to Lemma 2.13 . 
Lemma 7.2 Suppose $n_{a}=4$ and $n_{b} \geq 4$. If $\operatorname{val}\left(u_{1}, \hat{\Gamma}_{a}^{+}\right) \leq 1$ and $\hat{\Gamma}_{b}^{+}$has a boundary vertex $v_{j}$ of valence at most 3 , then $\Gamma_{a}$ is kleinian.

Proof. By Lemma $6.9 u_{1}$ is parallel to $u_{3}$ and antiparallel to $u_{2}, u_{4}$. Since $u_{1}$ is incident to at most 1 family of positive edges, it is incident to at least three negative $j$-edges at $u_{1}$, so $v_{j}$ has at least three positive edge endpoints labeled 1 . Hence $v_{j}$ being a boundary vertex implies that it has at least 9 positive edges. If $v_{j}$ is incident to 10 or more positive edges of $\Gamma_{b}$ then it has a family of 4 parallel positive edges and hence $\Gamma_{a}$ is kleinian. Therefore we may assume that it has exactly 9 positive edges, divided into three families of parallel edges, each family containing exactly three edges. See Figure 7.1.

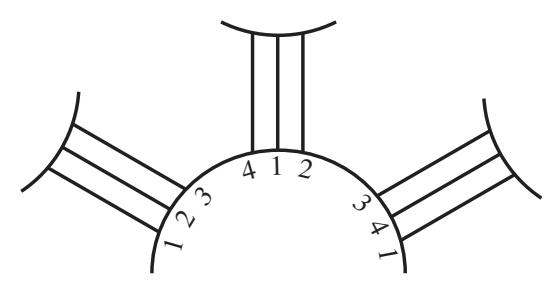

Figure 7.1

Since $n_{a}=4$, each of these families contains a Scharlemann bigon, so the labels of the middle edge in the family are labels of a Scharlemann bigon. It follows that 1,2,4 are labels of Scharlemann bigons. Thus if the result is not true then 3 is not a label of Scharlemann bigon, and $\Gamma_{b}$ contains both (12)- and (14)-Scharlemann bigons.

There are 7 adjacent negative edges at $v_{j}$, so three of then have labels 1 or 3 at $v_{j}$. These cannot all be parallel in $\Gamma_{a}$ as otherwise there would be three $j$-edges in a family and hence the family would contain more than $n_{b}$ edges, contradicting Lemma 2.3(1) and the fact that $\Gamma_{b}$ is not positive. On $\hat{\Gamma}_{a}^{+}$this implies that there are at least two edges with endpoints on $\left\{u_{1}, u_{3}\right\}$, hence $\operatorname{val}\left(u_{1}, \hat{\Gamma}_{a}^{+}\right) \leq 1$ implies that there is a loop $\hat{e}$ based at $u_{3}$. Since $\Gamma_{b}$ contains both (12)- and (14)-Scharlemann bigons, this is a contradiction to Lemma 7.1.

Lemma 7.3 Suppose $n_{a}=4$ and $n_{b} \geq 4$. If $\operatorname{val}\left(u_{i}, \hat{\Gamma}_{a}^{+}\right) \leq 1$ for some $i$ then $\Gamma_{a}$ is kleinian.

Proof. If $\Gamma_{b}$ is positive then $\Gamma_{a}$ is kleinian by Lemma $6.4(2)$. Therefore we may assume that $\Gamma_{b}$ is non-positive. By Lemmas 2.3(3) and 2.7(1) each family of parallel edges in $\Gamma_{a}$ contains at most $n_{b}$ edges. Also, notice that since $u_{i}$ is incident to more than $n_{b}$ negative edges, by Lemmas 2.8 and 2.2(4) the surface $\hat{F}_{a}$ is separating, hence $u_{i}$ is parallel to $u_{j}$ if and only if $i$ and $j$ have the same parity. 
Without loss of generality we may assume that $\operatorname{val}\left(u_{1}, \hat{\Gamma}_{a}^{+}\right) \leq 1$. Assume $\Gamma_{a}$ is not kleinian. Then by Lemma $7.2 \hat{\Gamma}_{b}^{+}$has no boundary vertex of valence 3 , and by Lemma 6.4(3) it has no interior vertex of valence at most 7 . Also, each vertex $v_{j}$ of $\hat{\Gamma}_{b}^{+}$has valence at least 3 because it is incident to at least three positive edges with label 1 at $v_{j}$, which by Lemma 2.3(3) must be mutually non-parallel. Therefore by Lemma 2.11 all vertices of $\hat{\Gamma}_{b}^{+}$are boundary vertices of valence 4 .

If $n_{b}>4$ then by Lemma 2.3(3) the family of positive edges at $u_{1}$ contains at most $n_{b} / 2+2<n_{b}$ edges, so some $v_{j}$ is incident to 4 positive edges with label 1 at $v_{j}$, which implies that $v_{j}$ has at least 13 positive edges in four families, so one of the families contains 4 edges and hence $\Gamma_{a}$ is kleinian by Lemma 6.4(1). Similarly if $\Delta>4$ then $\Gamma_{a}$ is kleinian.

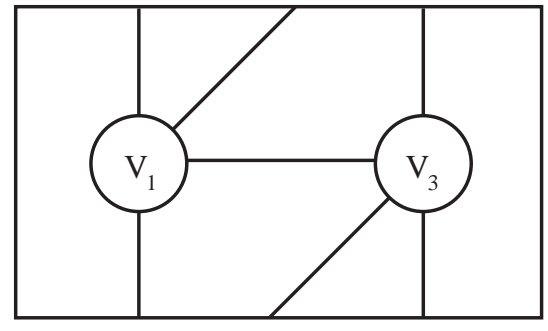

(a)

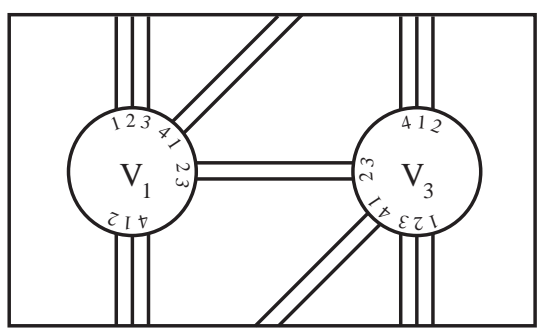

(b)

Figure 7.2

Now suppose $\Delta=n_{b}=4$. Then $\operatorname{val}\left(v_{j}, \hat{\Gamma}_{b}^{+}\right)=4$ for all $j$ implies that each component of $\hat{\Gamma}_{b}^{+}$has two loops and two non-loop edges, as shown in Figure 7.2(a). By the parity rule a loop based at $v_{j}$ has labels of different parity on its two endpoints, hence one sees that the number of positive edge endpoints of $\Gamma_{b}$ at each $v_{j}$ is even. By Lemma 6.4(4) we may assume that $v_{j}$ has less than 12 positive edges, hence the above implies that each $v_{j}$ is incident to exactly 10 positive edges. If some $v_{j}$ is incident to only one loop in $\Gamma_{b}$ then each of the non-loop family incident to $v_{j}$ contains 4 edges and we are done. If some $v_{j}$ is incident to two parallel loops in $\Gamma_{b}$ then they form a Scharlemann bigon with label pair $\{1,2\}$, say. Each of the two non-loop families contains three edges, hence the middle edge endpoint is a label of a Scharlemann bigon. Examining the labeling we see that all labels are Scharlemann bigon labels.

We now assume that each $v_{j}$ is incident to three parallel loop edges. See Figure 7.2(b). The two outermost loops form a Scharlemann bigon with 1 as one of its labels. There are 6 adjacent negative edges at $v_{1}$, so three of then have labels 1 or 3 at $v_{1}$. By the same argument as in the last paragraph of the proof of Lemma 7.2 we may assume that the two Scharlemann bigons at $v_{1}$ and $v_{3}$ have the same label pair (12). The labeling of edge endpoints around $v_{1}$ and $v_{3}$ in a component of $\Gamma_{b}^{+}$is now as shown in Figure 7.2(b). 
Because of the parity rule, the 4 non-loop edges cannot be divided into a family of 1 and another family of 3 edges, so they must form two pairs of parallel edges. From the labeling in Figure 7.2(b) one can see that they form two Scharlemann bigons with label pairs $\{2,3\}$ and $\{4,1\}$, respectively. The result now follows from Lemma 6.3.

We now assume that $\operatorname{val}\left(u_{1}, \hat{\Gamma}_{a}^{+}\right)=\operatorname{val}\left(u_{3}, \hat{\Gamma}_{a}^{+}\right)=2$. Then $\hat{\Gamma}_{a}^{+}$contains either a cycle $C$ containing both $u_{1}, u_{3}$, or it has two cycle components $C, C^{\prime}$ containing $u_{1}, u_{3}$, respectively.

Lemma 7.4 If $\operatorname{val}\left(u_{i}, \hat{\Gamma}_{a}^{+}\right)=2$ then $i$ is a label of a Scharlemann bigon in $\Gamma_{b}$.

Proof. Let $k$ be the number of interior vertices in $\hat{\Gamma}_{b}^{+}$. Let $m$ be the number of edges in $\hat{\Gamma}_{b}^{+}$. We claim that $m_{1} \leq 2 n_{b}+k$. Formally adding edges to $\hat{\Gamma}_{b}^{+}$ if necessary we may assume that any face $A$ between two adjacent components of $\hat{\Gamma}_{b}^{+}$is an annulus. It is easy to see that if $\partial A$ contains $p$ vertices then we can add $p$ edges to make each face on $A$ a triangle. Therefore we can add at least $n_{b}-k$ edges to $\hat{\Gamma}_{b}^{+}$to create a graph $G$ on the torus $\hat{F}_{b}$ whose faces are all triangles. By an Euler characteristic argument we see that $G$ has $3 n_{b}$ edges, hence $\hat{\Gamma}_{b}^{+}$has at most $3 n_{b}-\left(n_{b}-k\right)=2 n_{b}+k$ edges, and the claim follows.

Now let $m^{\prime}$ be the number of negative edges of $\Gamma_{a}$ incident to $u_{i}$. Note that if $m<m^{\prime}$ then two negative edges at $u_{i}$ are parallel in $\Gamma_{b}$ and we are done. By Lemma 2.3(3) each positive family $\hat{e}$ in $\Gamma_{a}$ contains at most $(n / 2)+2$ edges. Moreover, if $k>0$ then some label does not appear on endpoints of edges in $\hat{e}$, so $\hat{e}$ has at most $n_{b} / 2$ edges. Since $u_{i}$ is incident to two families of positive edges, we have $m^{\prime} \geq 4 n_{b}-2\left(n_{b} / 2\right)=3 n_{b}$ if $k>0$, and $m^{\prime} \geq 4 n_{b}-2\left(n_{b} / 2+2\right)=3 n_{b}-4$ if $k=0$. Since $m \leq 2 n_{b}+k$, we have $m<m^{\prime}$ (and hence $i$ is a label of a Scharlemann bigon in $\Gamma_{b}$ ), unless $k=0, n_{b}=4$ and $m=m^{\prime}=8$.

In this last case $\left(k=0, n_{b}=4\right.$ and $\left.m=m^{\prime}=8\right)$, all vertices of $\hat{\Gamma}_{b}^{+}$are boundary vertices, hence by Lemma 6.8 there is no jumping label at $u_{i}$. On the other hand, since $m^{\prime}=8$, each positive family at $u_{i}$ have 4 edges, so the two positive families cannot be adjacent by Lemma 6.6(2); hence there is a label $j$ such that the two negative edges labeled $j$ at $u_{i}$ are separated by the two positive edges labeled $j$ at $u_{i}$, so $j$ is a jumping label at $u_{i}$, which is a contradiction.

Lemma 7.5 If $\operatorname{val}\left(u_{1}, \hat{\Gamma}_{a}^{+}\right)=\operatorname{val}\left(u_{3}, \hat{\Gamma}_{a}^{+}\right)=2$ then $\Gamma_{a}$ is kleinian.

Proof. By Lemma $7.4 u_{1}, u_{3}$ are labels of Scharlemann bigons. If some vertex, say $u_{4}$, is not a label of Scharlemann bigon then there must be (12)- and (23)Scharlemann bigons in $\Gamma_{b}$. By Lemma 7.4 we have $\operatorname{val}\left(u_{4}, \hat{\Gamma}_{a}^{+}\right)>2$, so there is a loop edge $e$ of $\hat{\Gamma}_{a}^{+}$based at $u_{4}$. This is a contradiction to Lemma 7.1 (with labels permuted).

Proposition 7.6 If $\hat{\Gamma}_{a}^{+}$has a small component then (1) $\Gamma_{a}$ is kleinian, and (2) $\hat{\Gamma}_{a}^{+}$has at most 4 edges. 
Proof. Let $G$ be a small component of $\hat{\Gamma}_{a}^{+}$. If $G$ contains only one vertex $u_{1}$ and two edges then it cuts the torus into a disk containing the other three vertices. It is easy to see that in this case there is a vertex of valence at most 2 in $\hat{\Gamma}_{a}^{+}$, which by Lemma $2.3(3)$ is incident to at most $2 n_{b}$ edges, hence at least $2 n_{b}$ negative edges. By Lemma $2.8 \Gamma_{b}$ has a Scharlemann cycle, so the surface $\hat{F}_{a}$ is separating. Therefore $u_{3}$ is parallel to $u_{1}$ and is antiparallel to $u_{2}$ and $u_{4}$. It follows that $u_{3}$ is incident to no positive edges, so by Lemma $7.3 \Gamma_{a}$ is kleinian. If $G$ is not as above then either it contains a vertex of valence at most 1 , or it is a cycle, in which case (1) follows from Lemmas 7.3 and 7.5.

Since $\Gamma_{a}$ is kleinian, by Lemma 6.2(4) there is a free involution of $\Gamma_{a}$ sending $u_{i}$ to $u_{i+1}$, hence the number of edges ending at $\left\{u_{2}, u_{4}\right\}$ is the same as the number of edges ending at $\left\{u_{1}, u_{3}\right\}$, which is at most two in all cases discussed above. Hence (2) follows.

\section{If $n_{a}=4, n_{b} \geq 4$ and $\Gamma_{b}$ is non-positive then $\hat{\Gamma}_{a}^{+}$ has no small component}

Denote by $X$ the union of $\hat{\Gamma}_{b}^{+}$and all its disk faces.

Lemma 8.1 Suppose $n_{a}=4, n_{b} \geq 4, \Gamma_{b}$ is non-positive, and $\hat{\Gamma}_{a}^{+}$has a small component. Then

(1) each vertex of $\Gamma_{b}$ is incident to at most 8 negative edges;

(2) if $v_{j}$ is incident to more than 4 negative edges then $j$ is a label of a Scharlemann bigon;

(3) if $v_{j}$ is a boundary vertex of valence 3 in $\hat{\Gamma}_{b}^{+}$then it is incident to either 6 or 8 negative edges, and $j$ is a label of a Scharlemann bigon;

(4) $\operatorname{val}\left(v_{j}, \hat{\Gamma}_{b}^{+}\right) \geq 3$ if $v_{j}$ is a boundary vertex, and $\geq 2$ otherwise;

(5) each component of $X$ is either (a) a cyclic union of disks and (possibly) arcs, or (b) a cycle, or (c) an annulus.

Proof. Since $\hat{\Gamma}_{a}^{+}$has a small component, by Proposition $7.6 \Gamma_{a}$ is kleinian, and $\hat{\Gamma}_{a}^{+}$has at most 4 edges.

(1) If $v_{j}$ is incident to 9 negative edges then three of them are parallel on $\Gamma_{a}$ because $\hat{\Gamma}_{a}^{+}$has at most four edges, which contradicts Lemma 2.3(1).

(2) If $v_{j}$ is incident to 5 negative edges then two of them form a Scharlemann bigon in $\Gamma_{a}$ because $\hat{\Gamma}_{a}^{+}$has only four edges by Proposition 7.6.

(3) Since $\Gamma_{a}$ is kleinian, by Lemma 6.2(2) $v_{j}$ is incident to an even number of negative edges. Each family of positive edges contains at most four edges, and by Lemma 6.6 two adjacent families contain at most 6 edges, hence the three positive families at $v_{j}$ contain at most 10 edges. The result now follows from (1) and (2).

(4) By (1) $v_{j}$ is incident to at least 8 positive edges, which are divided into at least two families, and if two then they cannot be adjacent by Lemma 6.6(1). 
(5) If a component of $X$ is contained in a disk then by Lemma 2.9 it would have either a boundary vertex of valence at most 2 , which is impossible by (4), or six boundary vertices of valence 3 , which is a contradiction because by $(3)$ each such vertex is a label of Scharlemann bigon while by Lemma 2.3(4) $\hat{\Gamma}_{b}^{+}$has at most two labels of Scharlemann bigons for each sign. Therefore no component of $X$ is contained in a disk on the torus $\hat{F}_{b}$. Since $\Gamma_{b}$ is not positive, this implies that each component of $X$ is contained in an annulus but not a disk on $\hat{F}_{b}$.

If there is a sub-disk $D$ of $X$ such that $D \cap \overline{X-D}$ is a single point $v$ then either $\hat{\Gamma}_{b}^{+} \cap D$ contains a boundary vertex of valence 2 other than $v$, or 3 boundary vertices of valence 3 other than $v$, which again leads to a contradiction as above.

Let $X_{1}$ be a component of $X$, and let $v_{1}$ be a boundary vertex on the left cycle $C_{l}$ of $X_{1}$, as defined in Section 4 . Then there is another component $X_{2}$ of $X$ such that the annulus $A$ between $C_{l}$ and the right cycle $C_{r}^{\prime}$ of $X_{2}$ has interior disjoint from $\hat{\Gamma}_{b}^{+}$. Denote by $m_{j}$ the number of negative edges incident to $v_{j}$, and by $m^{\prime}=m_{1}^{\prime}$ the number of negative edges on $A$ which are not incident to $v_{1}$.

Lemma 8.2 Suppose $n_{a}=4, n_{b} \geq 4, \Gamma_{b}$ is non-positive, and $\hat{\Gamma}_{a}^{+}$has a small component. Let $v_{1}$ be a boundary vertex of $X_{1}$ with $m_{1}>4$. Then

(1) $v_{1}$ is a label of a Scharlemann bigon;

(2) $m^{\prime}=0$ if $m_{1}=8$;

(3) $m^{\prime} \leq 2$ if $m_{1}>4$;

(4) $C_{l}$ contains no other boundary vertices of valence at most 4.

Proof. (1) Since $\hat{\Gamma}_{a}^{+}$has only four edges, two of the negative edges at $v_{1}$ form a Scharlemann bigon on $\Gamma_{a}$.

(2) If $m_{1}=8$ then since $\hat{\Gamma}_{a}^{+}$has only 4 edges, the 8 negative edges at $v_{1}$ form 4 Scharlemann cocycles, which must all go to the same vertex $v_{2}$ on $C_{r}^{\prime}$ because $v_{1}$ is a boundary vertex and the cocycles are essential loops. These cocycles separate $C_{l}$ from $C_{r}^{\prime}$, hence all negative edges in $A$ incident to a vertex of $C_{l}-v_{1}$ must have the other endpoint on $v_{2}$. On the other hand, by Lemma $8.1(1) v_{2}$ is incident to at most 8 negative edges, and by the above all of them must connect $v_{2}$ to $v_{1}$. Hence $m^{\prime}=0$.

(3) By Proposition 7.6 $\Gamma_{a}$ is kleinian, so by Lemma 6.2(2) $m_{1}$ is even; hence by (2) we may assume that $m_{1}=6$. Since $\hat{\Gamma}_{a}^{+}$has only 4 edges, the 6 negative edges incident to $v_{1}$ contain at least 2 Scharlemann cocycles, which connect $v_{1}$ to some $v_{2}$ on $C_{r}^{\prime}$. If $v_{2}$ is incident to 8 negative edges in $A$ then as in (2) these edges form 4 Scharlemann cocycles, which must all connect to the same vertex $v_{1}$ and hence $m^{\prime}=0$. By Lemma 6.2(2) each family of parallel edges in $\Gamma_{b}$ has an even number of edges, so $v_{2}$ cannot be incident to 7 negative edges in $A$. If $v_{2}$ is incident to 6 or less negative edges in $A$ then by the above 4 of them connect to $v_{1}$, so there are at most 2 connecting to $C_{l}-v_{1}$, hence $m^{\prime} \leq 2$.

(4) A boundary vertex on $C_{l}-v_{1}$ of valence at most 4 in $\hat{\Gamma}_{b}^{+}$is incident to at most 12 positive edges by Lemma 6.6(2), and hence at least 4 negative edges, 
which must lie in $A$ because it is a boundary vertex on $C_{l}$. This is contradicts (3).

Lemma 8.3 Suppose $n_{a}=4, n_{b} \geq 4, \Gamma_{b}$ is non-positive, and $\hat{\Gamma}_{a}^{+}$has a small component. If a component $X_{1}$ of $X$ contains a boundary vertex $v_{1}$ of valence 3, then $X_{1}$ is an annulus containing exactly two vertices, both of which are of valence 3 and are labels of Scharlemann bigons.

Proof. By Lemma 6.6(2) $v_{1}$ is incident to at least 6 negative edges. Consider the three possible types of $X_{1}$ in Lemma 8.1(5). It cannot be a cycle because it has a boundary vertex $v_{1}$. If $X_{1}$ is an annulus or a cyclic union of disks and arcs then by Lemma 8.2(4), $C_{l}-v_{1}$ has no boundary vertex of valence at most 4 in $\hat{\Gamma}_{b}^{+}$, which implies that there is a boundary vertex $v_{3}$ of valence 3 on the right circle $C_{r}$ of $X_{1}$, hence for the same reason $C_{r}-v_{3}$ contains no boundary vertex of valence at most 4 . By Lemma 8.2(3) there are at most 4 negative edges incident to $C_{l} \cup C_{r}-\left\{v_{1}, v_{3}\right\}$, so there is no (non-boundary) vertex of valence 2 on $X_{1}$. Thus either

(i) $X_{1}$ is an annulus containing only the two vertices $v_{1}$ and $v_{3}$; or

(ii) $X_{1}$ is an annulus containing exactly four vertices and the other two are boundary vertices of valence 5 ; or

(iii) $X_{1}$ is as in Figure 8.1 (a) or (b).

Case (i) gives the conclusion of the lemma because, as in the proof of Lemma 8.2 , a boundary vertex of valence 3 must be a label of a Scharlemann bigon. We need to show that (ii) and (iii) are impossible.

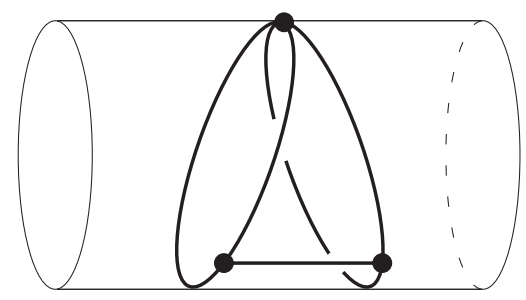

(a)

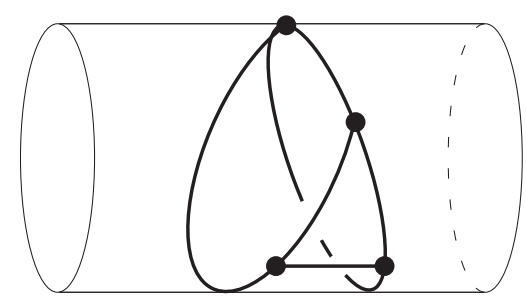

(b)

Figure 8.1

In case (ii), let $v_{5}, v_{7}$ be the boundary vertices of valance 5 on $X_{1}$, with $v_{5} \subset C_{l}$. Note that all faces of $X_{1}$ are triangles. If $v_{1}$ had 8 negative edges then by Lemma 8.2(2) $v_{5}$ would have no negative edge, which is impossible by Lemma $6.4(3)$. Therefore $v_{1}$ has exactly 6 negative edges and 10 positive edges, so by Lemma 6.6(1) the weights of the edges of $X_{1}$ incident to $v_{1}$ must be $(4,2,4)$. Now the middle edge of $X_{1}$ at $v_{5}$ has weight 2 and the two boundary edges have weight 4 , so again by Lemma $6.6(1)$ the weights around $v_{5}$ must be $(4,2,2,2,4)$. By Lemma 6.7(2) the edges of weight 2 must be non-Scharlemann. This is a 
contradiction because a triangle with a corner at $v_{5}$ bounded by two weight 2 edges has two non-Scharlemann bigons on its boundary while by Lemma 6.7(1) it has only one.

In case (iii), we assume $X_{1}$ is as in Figure 8.1(a). The other case is similar. Let $v_{1}, v_{3}$ be the vertices of valence 3 in the figure, and let $v_{5}$ be the other vertex. If $m_{1}=8$ then by Lemma $8.2(2) v_{5}$ is incident to no negative edges on the side of $C_{l}$, and at most 2 negative edges on the side of $C_{r}$. Therefore the four positive edges of $\hat{\Gamma}_{b}^{+}$at $v_{5}$ are adjacent to each other, representing a total of 14 edges. It follows that there are two adjacent families of positive edges, each containing 4 edges, which is a contradiction to Lemma 6.6(1). If $m_{1}=m_{3}=6$ then each of $v_{1}$ and $v_{3}$ is incident to 10 positive edges, so by Lemma 6.6(1) the weights of the edges of $\hat{\Gamma}_{b}^{+}$at $v_{1}$ and $v_{3}$ are $(4,2,4)$, in which case $v_{5}$ again has two adjacent families of 4 positive edges each, contradicting Lemma 6.6(1).

Lemma 8.4 Suppose $n_{a}=4, n_{b} \geq 4, \Gamma_{b}$ is non-positive, and $\hat{\Gamma}_{a}^{+}$has a small component. If a component $X_{1}$ of $X$ does not contain a boundary vertex of valence 3 , then

(1) $X_{1}$ is either a cycle or an annulus containing exactly two vertices; and

(2) all vertices of $X_{1}$ are labels of Scharlemann bigons.

Proof. By Lemma 6.6(3) all interior vertices of $X$ have valence at least 6 . Since $X_{1}$ has no boundary vertex of valence 3 , by Lemma 2.11 it is either a cycle, or an annulus with all interior vertices of valence 6 , all boundary vertices of valence 4 , and all faces triangles. The result is true when $X_{1}$ is a cycle because any vertex of valence 2 has more than 4 negative edges and hence is a label of Scharlemann cycle. Therefore we assume that $X_{1}$ is an annulus. If $X_{1}$ has an interior vertex $v_{1}$ then by Lemmas 6.6(4) the weights of the edges of $X_{1}$ around $v_{1}$ must be $(4,2,4,2,2,2)$ and any edge of weight 2 represents a non-Scharlemann bigon. Thus the triangle with a corner at $v_{1}$ bounded by two weight 2 edges has the property that it has at least two edges representing non-Scharlemann bigons, which is a contradiction to Lemma 6.7(1). Therefore $X_{1}$ has no interior vertex.

First assume that some vertex $v_{1}$ on the left cycle $C_{l}$ of $X_{1}$ is incident to less than 12 positive edges, and hence more than 4 negative edges. Since all vertices of $X_{1}$ are of valence 4 in $\hat{\Gamma}_{b}^{+}$, by Lemma $8.2(3) C_{l}$ has no other vertices. In this case $C_{r}$ also contains only a single vertex $v_{3}$. By Lemma 6.6(2) $v_{3}$ cannot have more than 12 positive edges, and if 12 then the weights around it are $(4,2,4,2)$. However this cannot happen because the first and the last numbers are the weights of the loop edge at $v_{3}$ and hence must be the same. Therefore $v_{3}$ has less than 12 positive edges. Now by Lemma 8.1(2) $v_{3}$ and $v_{1}$ are labels of Scharlemann bigons, and the result follows.

Now assume that each vertex of $X_{1}$ is incident to 12 positive edges. This implies that the weights of the edges of $X_{1}$ around each vertex are either $(4,2,4,2)$ or $(2,4,2,4)$, so any pair of adjacent edges have different weights. However, this is impossible because two of the three edges of a triangle face must have the same weight. 
Lemma 8.5 Suppose $n_{a}=4, n_{b} \geq 4, \Gamma_{b}$ is non-positive, and $\hat{\Gamma}_{a}^{+}$has a small component. Then

(1) $n_{b}=4$.

(2) $\operatorname{val}\left(u_{i}, \hat{\Gamma}_{a}^{+}\right) \geq 2$ for all $i$.

(3) Each component of $\hat{\Gamma}_{a}^{+}$is a loop.

Proof. (1) By Lemmas 8.3 and 8.4 each vertex of $\Gamma_{b}$ is a label of a Scharlemann bigon, and by Lemma 2.3(4) there are at most 4 such labels. Hence $n_{b}=4$.

(2) Suppose $u_{1}$ is incident to at most one edge of $\hat{\Gamma}_{a}^{+}$. By Lemmas 8.3 and 8.4 each component $G$ of $\hat{\Gamma}_{b}^{+}$consists of either (i) a cycle, or (ii) two loops and one non-loop edge, or (iii) two loops and two non-loop edges. Since there are at least 3 negative $j$-labels at $u_{1}$, there are at least 3 positive 1 -labels at each $v_{j}$, hence (i) cannot happen. Moreover, since each $v_{j}$ is a boundary vertex containing at least three positive 1-labels, it has more than 8 positive edges.

Suppose $G$ is of type (ii). Then the label 1 appears three times on positive edge endpoints around each of the two vertices of $G$, hence it appears a total of 6 times among the three families of positive edges in $G$, so by Lemmas 2.4 and 6.2(3) there is a (12)-Scharlemann bigon among each of these families. Since a loop and a non-loop edge cannot be parallel in $\Gamma_{a}$ (Lemma 2.3(5)), these represents at least four edges of $\hat{\Gamma}_{a}$ connecting $u_{1}$ to $u_{2}$, which cut the torus $\hat{F}_{a}$ into two disks. On the other hand, by the parity rule a loop at a vertex $v$ of $G$ must have labels of different parity on its two endpoints, so the total number of positive edges at $v$ is at least 10, divided into three families, hence one of the families has four parallel edges, which contains a (34)-Scharlemann bigon, giving a pair of edges on $\Gamma_{a}$ lying in the interior of the disks above which must therefore be parallel. This is a contradiction to the fact that a Scharlemann cocycle is essential (Lemma 2.2(5)).

Now suppose $G$ is of type (iii). If some $v_{j}$ is incident to 12 positive edges then by Lemma 6.6(1) the weights of the positive edges around $v_{j}$ are $(4,2,4,2)$, which is impossible because the first and the last weights are for a loop and hence must be the same. Since $v_{j}$ is incident to more than 8 positive edges, it is incident to exactly 10 positive edges, so the weights are $(2,4,2,2)$ or $(2,2,4,2)$ around each vertex. The two loops must be (12)-Scharlemann bigons in order for each vertex to have 3 edge endpoints labeled 1 . This completely determines the labeling of the edge endpoints up to symmetry. Examining the labeling one can see that the family of 4 parallel edges form an extended Scharlemann cycle, which is a contradiction to Lemma 2.2(6).

(3) By Proposition 7.6 $\Gamma_{a}$ is kleinian, hence the torus $\hat{F}_{a}$ is separating, so each edge of $\hat{\Gamma}_{a}^{+}$has endpoints on vertices whose subscripts have the same parity. By (2) a small component of $\hat{\Gamma}_{a}^{+}$must be a loop $C$. Let $u_{1}$ be a vertex of $C$. If $C$ does not contain $u_{3}$ then the component of $\hat{\Gamma}_{a}^{+}$containing $u_{3}$ must also be a loop because it contains no other vertices, and it cannot contain more than one edge as otherwise some component of $\hat{\Gamma}_{a}^{+}$would lie in a disk and hence would have a vertex of valence at most 1 , contradicting (2). Thus the graph $G$ consisting of $u_{1}, u_{3}$ and all edges with endpoints on them is either one loop or two disjoint loops. By Lemma 6.2(4) there is a involution of $\hat{\Gamma}_{a}^{+}$mapping $u_{1}$ and $u_{3}$ to $u_{2}$ 
and $u_{4}$ respectively, hence it maps $G$ to $\hat{\Gamma}_{a}^{+}-G$. Therefore the components in $\hat{\Gamma}_{a}^{+}-G$ are also loops.

Proposition 8.6 Suppose $n_{a}=4, n_{b} \geq 4$, and $\Gamma_{b}$ is non-positive. Then $\hat{\Gamma}_{a}^{+}$ has no small component.

Proof. Suppose to the contrary that $\hat{\Gamma}_{a}^{+}$has a small component. By Lemma 8.5 we have $n_{b}=4$ and each component of $\hat{\Gamma}_{a}^{+}$is a loop. Thus each $u_{i}$ is incident to at most 8 positive edges, so $\Gamma_{a}$ has no more positive edges than negative edges.

By Lemmas 8.3 and 8.4, each component $X_{1}$ of $X$ is either a circle or an annulus containing two vertices of $\hat{\Gamma}_{b}^{+}$. First assume that $X_{1}$ is a circle, so it is a small component of $\hat{\Gamma}_{b}^{+}$. Applying Proposition 7.6 and Lemma 8.5 with $\Gamma_{a}$ and $\Gamma_{b}$ reversed, we see that $\Gamma_{b}$ is kleinian, and all components of $\hat{\Gamma}_{b}^{+}$are also circles, hence $\Gamma_{b}$ also has the property that it has no more positive edges than negative edges. Applying the parity rule we see that both graphs have the same number of positive edges and negative edges. In particular, each family of positive edges contains exactly 4 edges, which by Lemma 6.2(3) must consist of a (12)-Scharlemann bigon and a (34)-Scharlemann bigon. Dually it implies that all negative edges connect $u_{1}$ to $u_{2}$ or $u_{3}$ to $u_{4}$, so there are 4 families of negative edges, each containing exactly 4 edges. Now the two positive families at $u_{1}$ contain 4 edges each, and, whether separated by the two negative families or not, their endpoints at $u_{1}$ have the same label sequence. This contradicts Lemma 6.6(1).

We may now assume that $X$ consists of two annular components $X_{1}, X_{2}$, each containing two vertices. Assume $v_{1}, v_{3} \in X_{1}$. As in the last paragraph of the proof of Lemma 8.5(2), in this case each vertex of $\Gamma_{b}$ is incident to 8 or 10 positive edges, therefore by Lemma 8.1(2) each vertex is a label of Scharlemann bigon, hence $\Gamma_{b}$ is also kleinian.

If $\operatorname{val}\left(v_{1}, \Gamma_{b}^{+}\right)=10$ then $v_{1}$ is incident to 6 negative edges, which are divided into two families of parallel edges on the annulus bounded by the loops at $v_{1}$ and $v_{2}$. Since $\Gamma_{b}$ is kleinian, each family contains an even number of edges, hence the number of edges in these two families are 4 and 2, respectively. Examining the labels at the endpoints of these edges, we see that two edges with the same label $i$ at $v_{1}$ have different labels at $v_{2}$. On $\Gamma_{a}$ this means that there are both loop and non-loop positive edges incident to $u_{i}$, which is a contradiction to the fact that $\hat{\Gamma}_{a}^{+}$consists of cycles only.

We have shown that $\operatorname{val}\left(v_{j}, \Gamma_{b}^{+}\right)=8$ for all $j$. Thus there are 16 positive edges on $\Gamma_{a}$, so each of the two positive families incident to $u_{1}$ contains 4 edges. Since all vertices of $\Gamma_{b}$ are boundary vertices, there is no jumping label on any vertex of $\Gamma_{b}$, hence by Lemma 6.8 there is no jumping label at $u_{1}$, so the two families of positive edges must be adjacent. This is a contradiction to Lemma $6.6(2)$. 


\section{If $\Gamma_{b}$ is non-positive and $n_{a}=4$ then $n_{b} \leq 4$}

Note that if $\Gamma_{a}$ is positive then each vertex of $\Gamma_{b}$ is a label of a Scharlemann bigon and hence $n_{b} \leq 4$ by Lemma 2.3(4). By Proposition 8.6 the statement in the title is true if $\hat{\Gamma}_{a}^{+}$has a small component. Therefore we may assume that $\hat{\Gamma}_{a}^{+}$consists of two large components $G_{1}$ and $G_{2}$, each of which must be one of the graphs of type (3), (9) or (10) in Figure 4.2. As before, denote by $X_{a}$ the union of $\hat{\Gamma}_{a}^{+}$and all its disk faces, and by $X_{i}$ the components of $X_{a}$ containing $G_{i}, i=1,2$. Denote $n=n_{b}$.

Lemma 9.1 Suppose that $\Gamma_{b}$ is non-positive, $n_{a}=4$ and $n>4$. Then $\hat{\Gamma}_{b}^{+}$ contains no interior vertex.

Proof. Otherwise $\Gamma_{b}$ has a vertex $v_{i}$ which is incident to positive edges only. By Lemma 2.3(1) no three of these edges are parallel on $\Gamma_{a}$, so $\hat{\Gamma}_{a}$ contains at least $\Delta n_{a} / 2 \geq 8$ negative edges, and hence at most $3 n_{a}-8=4$ positive edges by Lemma 2.5 , so $\hat{\Gamma}_{a}^{+}$has a small component, contradicting our assumption.

Lemma 9.2 Suppose that $\Gamma_{b}$ is non-positive, $n_{a}=4$ and $n>4$. Suppose $X_{a}$ is a disjoint union of two annuli. Let $G$ be the subgraph of $\Gamma_{b}$ consisting of positive 1-edges and all vertices. Then $G$ cannot have two triangle faces $D_{1}, D_{2}$ with an edge in common.

Proof. Since $X_{a}$ is a disjoint union of two annuli, all negative edges of $\Gamma_{a}$ incident to $u_{1}$ must have the other endpoint on the same vertex, say $u_{2}$, and vice versa. On $G$ this means that every edge has label pair (12), and all positive edges with an endpoint labeled 1 or 2 are in $G$. Thus no edge in the interior of $D_{i}$ has label 1 or 2 at any of its endpoints. Up to symmetry the labels on the boundary of the two triangles must be as shown in Figure 9.1. Since the labels 3 and 4 must appear between two label 1 at a vertex, one of the triangles, say $D_{1}$, must contain some (34) edges. Since all the vertices are parallel, one can see that the labels 3 and 4 appear at each corner of $D_{1}$, hence there are three edges inside of $D_{1}$. Since there is no trivial loop, they must form an extended Scharlemann cycle, contradicting Lemma 2.2(6).

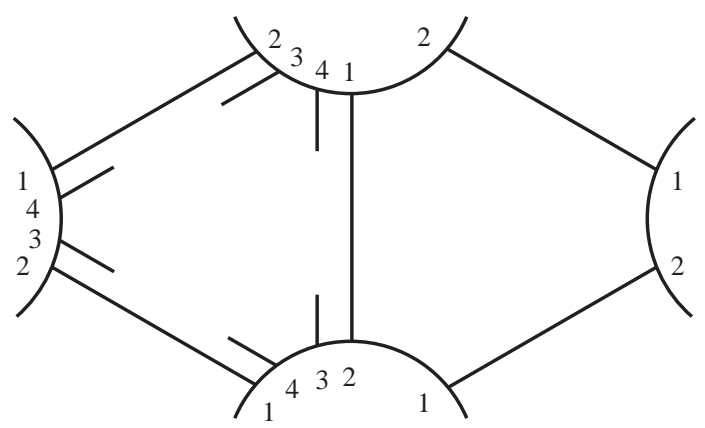


Figure 9.1

Lemma 9.3 Suppose that $\Gamma_{b}$ is non-positive, $n_{a}=4$ and $n>4$. If $X_{a}$ is a disjoint union of two annuli then $\Gamma_{a}$ is kleinian.

Proof. By Lemma 6.3 it suffices to show that each vertex $u_{i}$ of $\Gamma_{a}$ is a label of a Scharlemann bigon. Let $t_{i}$ be the number of negative edges at $u_{i}$. Since there are at most two families of negative edges incident to $u_{i}$, we have $t_{i} \leq 2 n$ by Lemma 2.3(1). On the other hand, since $u_{i}$ is a boundary vertex of valence at most 4 in $\hat{\Gamma}_{a}^{+}$, by Lemma 2.7 it is incident to at most $2(n+2)=2 n+4$ positive edges. If there are $2 n+4$ then $u_{i}$ is incident to at least four loops and the four outermost loops form an extended Scharlemann cycle, which is impossible by Lemma 2.2(6). Also by the parity rule the loops have labels of different parity at its two endpoints, hence the number of positive edges at $u_{i}$ must be even. It follows that $u_{i}$ is incident to either $2 n$ or $2 n+2$ positive edges, hence $t_{i}=2 n$ or $2 n-2$.

Assume that $u_{1}$ is not a label of a Scharlemann bigon. Then the negative edges at $u_{1}$ are mutually non-parallel positive edges in $\Gamma_{b}$, hence $\hat{\Gamma}_{b}^{+}$has at least $2 n-2$ edges. Denote by $G$ the subgraph of $\Gamma_{b}$ consisting of positive 1-edges. Let $Y$ be the union of $G$ and its disk faces, and let $k$ be the number of boundary vertices of $G$.

First assume $t_{1}=2 n$. By Lemma $9.1 G$ has no interior vertex, and clearly it has no isolated vertex, so we can apply Lemma 2.10(1) to conclude that $k \geq t_{1}-n=n$. Since $G$ only has $n$ vertices, we must have $k=n$, so all vertices of $G$ are boundary vertices, and hence there is no cut vertex. In this case $Y$ contains exactly $n$ boundary edges, so it has at least one (actually $n$ ) interior edge $e$. Since equality holds for the above inequality, by Lemma 2.10(1) all faces of $Y$ are triangles. Therefore $e$ is the common edge of two adjacent triangle faces, which is a contradiction to Lemma 9.2. Therefore this case is impossible.

Now assume $t_{1}=2 n-2$. In this case the two outermost loops at $u_{1}$ form a Scharlemann bigon, so by Lemma 2.2(4) $\hat{F}_{b}$ is separating, hence two vertices of $\Gamma_{b}$ are parallel if and only if they have the same parity. Therefore we can define $G_{1}$ (resp. $G_{2}$ ) to be the union of the components of $G$ containing $v_{i}$ with odd (resp. even) $i$. Similarly for $Y_{1}$ and $Y_{2}$. Then $G_{1}$ contains all the negative edges at $u_{1}$ with odd labels, and $G_{2}$ those with even labels. Therefore each $G_{i}$ contains exactly $n-1$ edges.

The $2 n+2$ positive edges at $u_{1}$ form at least $n+1$ negative edges in $\hat{\Gamma}_{b}$ because any family of $\Gamma_{b}$ contains at most 2 such edges. Hence $\hat{\Gamma}_{b}$ contains at least $(n+1)+(2 n-2)=3 n-1$ edges. Since a reduced graph on a torus contains at most $3 n$ edges (Lemma 2.5), we may add at most one edge to make the faces of the graph all triangles. Hence $\hat{\Gamma}_{b}$ has at most one 4-gon and all other faces are triangles. In particular, one of the $G_{i}$, say $G_{1}$, has the property that all its faces are triangles.

Let $V$ and $E$ be the number of vertices and edges of $G_{1}$, and let $E_{b}, V_{b}$ be the number of non-interior edges and boundary vertices, respectively. Note that 
$V-V_{b}$ is the number of cut vertices, and $E-E_{b}$ is the number of interior edges. We have shown that $V=n / 2$ and $E=n-1$.

By Lemma 2.10(1) we have $V_{b} \geq E-V=(n-1)-n / 2$, hence $G$ has $V-V_{b} \leq V-(E-V)=(n / 2)-(n-1-n / 2)=1$ cut vertex. If there is no cut vertex then the number of non-interior edges is the same as the number of vertices $V$, i.e. $E_{b}=V$. If it has a cut vertex $v$ then the equality $V_{b}=E-V$ holds, so by Lemma 2.10(1), $v$ has exactly two corners not on disk faces, which implies $v$ is incident to at most 4 non-interior edges, while every other vertex is incident to exactly two non-interior edges, hence $E_{b} \leq V+1$. In either case we have $E-E_{b} \geq E-(V+1)=(n-1)-(n / 2+1) \geq 1$, so $G$ has at least one interior edge $e$. Since all faces of $Y_{1}$ are triangles, $e$ is incident to two triangle faces of $G_{1}$, which is a contradiction to Lemma 9.2.

Lemma 9.4 Suppose that $\Gamma_{b}$ is non-positive, $n_{a}=4$ and $n>4$. Then $X_{a}$ is not a disjoint union of two annuli.

Proof. Assume to the contrary that $X_{a}$ is a union of two annuli. Let $t_{i}$ be the number of negative edges incident to $u_{i}$. As in the proof of Lemma 9.3, we have $t_{i}=2 n-2$ or $2 n$.

First assume that $t_{1}=2 n-2$. Let $\hat{e}_{1}, \hat{e}_{2}$ be the two families of edges in $\Gamma_{a}$ connecting $u_{1}$ to $u_{2}$. Note that a (12)-Scharlemann bigon in $\Gamma_{b}$ must have one edge in each of $\hat{e}_{1}$ and $\hat{e}_{2}$. By Lemma $9.3 \Gamma_{a}$ is kleinian, so all (12)-edges belong to Scharlemann bigons in $\Gamma_{b}$, hence each edge $e_{i}$ in $\hat{e}_{1}$ is parallel in $\Gamma_{b}$ to an edge $e_{i}^{\prime}$ in $\hat{e}_{2}$, and the label of $e_{i}$ at $u_{1}$ is the same as that of $e_{i}^{\prime}$ at $u_{2}$. In particular, $\hat{e}_{1}$ and $\hat{e}_{2}$ have the same number of edges, hence each contains exactly $n-1$ edges. Without loss of generality we may assume that the label $n$ does not appear at the endpoints at $u_{1}$ of edges in $\hat{e}_{1}$. By the above, $n$ does not appear at the endpoints at $u_{2}$ of edges of $\hat{e}_{2}$, hence the labels must be as in Figure 9.2. However, in this case the edge labeled 1 at $u_{1}$ has its other endpoint labeled $n$, which is a contradiction to the parity rule.

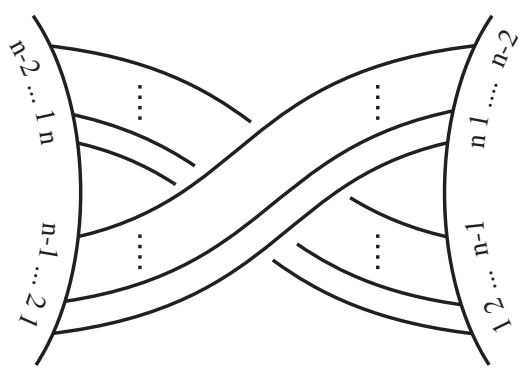

Figure 9.2

We now assume $t_{1}=2 n$. Then the two families of negative edges from $u_{1}$ to $u_{2}$ have the same transition function $\varphi$. Since there is a (12)-Scharlemann 
bigon, $\varphi^{2}=i d$, so the length of each $\varphi$-cycle is 1 or 2 . Since $n>4$, it follows that the edges of $\hat{e}_{1}$ form at least 3 cycles on $\Gamma_{b}$, which is a contradiction to Lemma $2.14(2)$.

Lemma 9.5 Suppose that $\Gamma_{b}$ is non-positive, $n_{a}=4$ and $n>4$. If $G_{1}$ is of type (3), then $G_{2}$ is also of type (3), and the two loops of $\hat{\Gamma}_{a}^{+}$do not separate the two vertices $u_{3}, u_{4}$ which are not on the loops.

Proof. Let $u_{3}$ be the vertex of $G_{1}$ which has valence 2 . If $G_{2}$ is not of type (3), or if the two loops $\hat{e}_{1} \cup \hat{e}_{2}$ of $\hat{\Gamma}_{a}^{+}$separates $u_{3}$ and $u_{4}$ then all negative edges incident to $u_{3}$ have their other endpoint on the same vertex $u_{2}$ of $G_{2}$, and there are only two such families. Hence $u_{3}$ is incident to only four families of parallel edges, so by Lemmas 2.3(1) and 2.3(3) we must have $n=4$ and each family contains exactly four edges. Since the two families of positive edges are adjacent, this contradicts Lemma 6.6(1).

Lemma 9.6 Suppose that $\Gamma_{b}$ is non-positive, $n_{a}=4$ and $n>4$. Then $\hat{\Gamma}_{a}^{+}$ cannot be a union of two type (3) components.

Proof. Suppose that $\hat{\Gamma}_{a}^{+}$is a union of two type (3) components, so $\hat{\Gamma}_{a}$ has 6 positive edges $\hat{e}_{1}, \ldots, \hat{e}_{6}$. By Lemma 9.5 the two loops do not separate the two vertices which are not on the loops, so the edges appear as in Figure 9.3. By Lemmas 2.3(1), 2.3(3) and 6.6(1) each vertex has valence at least 5, so there is one edge $\hat{e}_{7}$ from $u_{2}$ to $u_{3}$, two edges $\hat{e}_{8}, \hat{e}_{9}$ from $u_{1}$ to $u_{2}$, and one edge $\hat{e}_{10}$ from $u_{1}$ to $u_{4}$. There are one or two edges $\hat{e}_{11}$ and $\hat{e}_{12}$ connecting $u_{3}$ to $u_{4}$. See Figure 9.3.

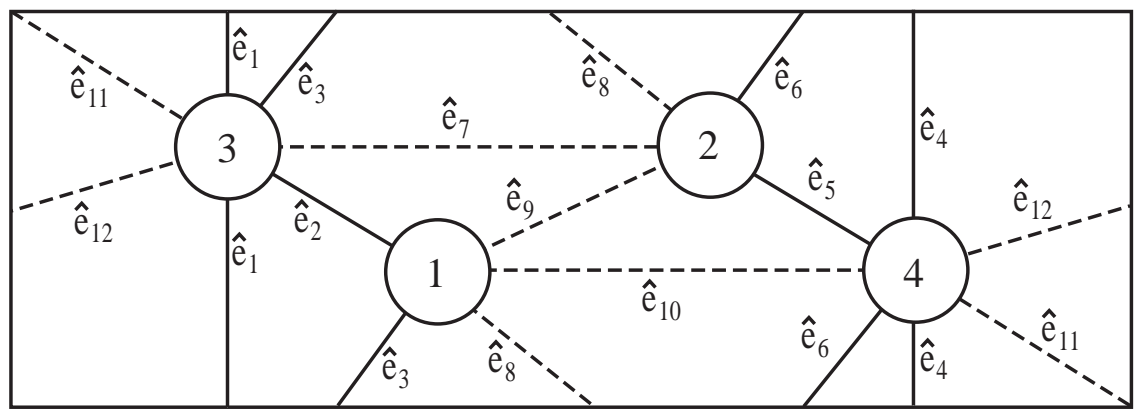

Figure 9.3

Denote by $w_{i}$ the number of edges in $\hat{e}_{i}$. By Lemma 2.7(2) the two positive edges $\hat{e}_{2}, \hat{e}_{3}$ contain at most $n+2$ edges, and the three negative families at $u_{1}$ contains at most $3 n$ edges, hence $\Delta=4$. Since each family contains at most $n$ 
edges, we have the following inequalities.

$$
\begin{gathered}
n \leq w_{2}+w_{3} \leq n+2 \\
3 n-2 \leq w_{8}+w_{9}+w_{10} \leq 3 n \\
n-2 \leq w_{i} \leq n \quad \text { for } i=7,8,9,10
\end{gathered}
$$

Since $u_{1}$ has at least $n$ adjacent positive edge endpoints and $n$ adjacent negative edge endpoints, each vertex of $\Gamma_{b}$ is incident to a positive edge and a negative edge, hence $\hat{\Gamma}_{b}^{+}$has no isolated or interior vertex. labels.

Claim 1. $\hat{\Gamma}_{b}^{+}$has at least $2 n-2$ edges, hence $\Gamma_{a}$ has at most two jumping

Let $e \in \hat{e}_{8}$, and $e^{\prime} \in \hat{e}_{10}$. Then $e$ and $e^{\prime}$ are not parallel in $\Gamma_{b}$ because if they were then they would form a Scharlemann bigon and hence have the same label pair on their endpoints, which is not the case because their label pairs are $\{1,2\}$ and $\{1,4\}$ respectively. Therefore the number of edges in $\hat{\Gamma}_{b}^{+}$is at least

$$
w_{8}+w_{10} \geq 3 n-2-w_{9} \geq 2 n-2
$$

Since $\hat{\Gamma}_{b}^{+}$has no interior or isolated vertex, by Lemma 2.10(1) it has at most $2 n-(2 n-2)=2$ non-boundary vertices. Since these are the only vertices containing jumping labels, by Lemma 2.18 they are the only possible jumping labels of $\Gamma_{a}$.

Claim 2. $w_{11}+w_{12} \geq 2 n-2$.

Note that since $w_{9}, w_{10} \leq n$, we have

$$
\begin{gathered}
8 n \\
=\operatorname{val}\left(u_{1}, \Gamma_{a}\right)+\operatorname{val}\left(u_{2}, \Gamma_{a}\right) \\
=\left(w_{2}+w_{3}+w_{10}\right)+\left(w_{5}+w_{6}+w_{7}\right)+2\left(w_{8}+w_{9}\right) \\
\leq\left(w_{2}+w_{3}+w_{7}\right)+\left(w_{5}+w_{6}+w_{10}\right)+4 n
\end{gathered}
$$

Thus either $w_{2}+w_{3}+w_{7} \geq 2 n$ or $w_{5}+w_{6}+w_{10} \geq 2 n$. Because of symmetry we may assume

$$
w_{2}+w_{3}+w_{7} \geq 2 n
$$

Divide the edge endpoints on $\partial u_{3}$ into $P_{1}, P_{2}, P_{3}, P_{4}$, as shown in Figure 9.4. Denote by $k_{i}$ the number of edge endpoints in $P_{i}$. A label that appears twice in one of the $P_{i}$ will be called a repeated label. Note that if $P_{i}$ contains a repeated label then $k_{i}>n$. Note also that a non-jumping label is a repeated label. Thus by Claim 1 there are at least $n-2$ repeated labels among all the $P_{i}$. Since $k_{1}=w_{7} \leq n$, there is no repeated label in $P_{1}$. 


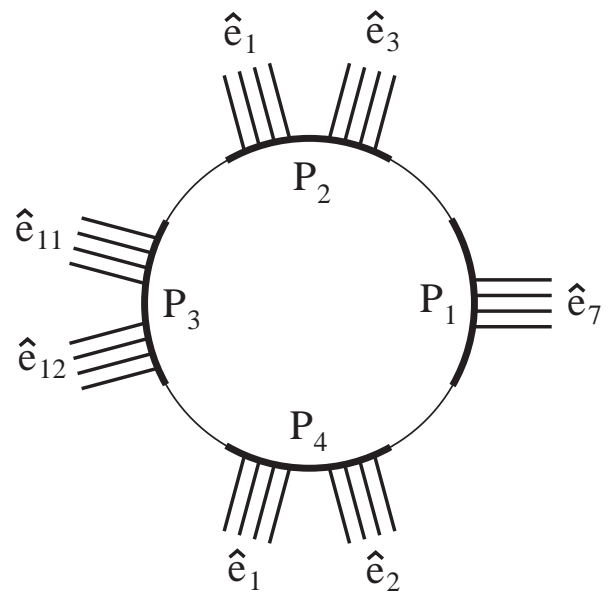

Figure 9.4

First assume that $k_{3}>n$. If both $k_{2}, k_{4} \leq n$ then all repeated labels are in $P_{3}$, so $k_{3} \geq 2 n-2$ and we are done. Because of symmetry we may now assume to the contrary that $k_{2}>n$. Thus one of $\hat{e}_{1}, \hat{e}_{3}$ contains more than $n / 2$ edges, so by Lemmas 2.4 and 2.2(4) $n$ is even, hence $n \geq 6$. Note that in this case $k_{4} \leq n$, as otherwise we would have $4 n=\sum k_{i} \geq(n-2)+3(n+1)>4 n$, a contradiction. Thus $P_{2}, P_{3}$ contain all the repeated labels and there are at least $n-2$ of them, so $k_{2}+k_{3} \geq 3 n-2$. Since $k_{2}=w_{1}+w_{3} \geq n+1$ and $w_{3} \leq(n / 2)+2$, we have $w_{1} \geq(n / 2)-1$, so $w_{3}-w_{1} \leq 3$. By equation (4) above, we have

$$
\begin{gathered}
4 n=\operatorname{val}\left(u_{3}, \Gamma_{a}\right)=w_{7}+k_{2}+k_{3}+\left(w_{1}+w_{2}\right) \\
=\left(k_{2}+k_{3}\right)+\left(w_{7}+w_{3}+w_{2}\right)-\left(w_{3}-w_{1}\right) \\
\geq(3 n-2)+2 n-3=5 n-5>4 n
\end{gathered}
$$

This is a contradiction, which completes the proof for the case $k_{3}>n$.

We now assume $k_{3} \leq n$. Then each non-jumping label is a repeated label in either $P_{2}$ or $P_{4}$. By Lemma 2.7(2) we have $k_{2}, k_{4} \leq n+2$, so each of $P_{2}, P_{4}$ contains at most 2 repeated labels. Since there are at least $n-2$ repeated labels, we have $n-2 \leq 4$, i.e., $n \leq 6$. As above, $P_{2}$ or $P_{4}$ containing a repeated label implies that $n$ is even, so $n=6$. In this case $k_{2}=k_{4}=8$, so by Lemma 2.3(3) each of $\hat{e}_{1}, \hat{e}_{2}, \hat{e}_{3}$ contains exactly 4 edges.

By equation (3) above we have $n-2 \leq k_{1}=w_{7} \leq n$. If $k_{1}=n-1$ or $n-2$ then the labels that do not appear in $P_{1}$ are repeated labels of both $P_{2}$ and $P_{4}$, so there are at most 3 distinct repeated labels, and hence at least $n-3=3$ jumping labels, contradicting Claim 1 . If $k_{1}=n$ then $k_{1}+k_{2}+k_{4}=3 n+4$, so the endpoints of the 4 edges of $\hat{e}_{1}$ at $P_{2}$ have the same label sequence as those of $\hat{e}_{1}$ at $P_{4}$. Hence these edges form an extended Scharlemann cycle, which is impossible by Lemma 2.2(6). 
Claim 3. $\Gamma_{a}$ is kleinian.

Clearly each of $u_{1}, u_{2}$ is incident to more than $2 n$ edges. Since $w_{7}+w_{8}+w_{9}=$ $4 n-\left(w_{5}+w_{6}\right) \geq 3 n-2$, we have $w_{7} \geq n-2$. By Claim $2 w_{11}+w_{12} \geq 2 n-2$, hence $w_{11}+w_{12}+w_{7} \geq 3 n-4>2 n$, so $u_{3}$ is also incident to more than $n$ negative edges. Similarly for $u_{4}$. By Lemmas 9.1 and 2.10(2) $\hat{\Gamma}_{b}^{+}$has at most $2 n$ edges, so two of the negative edges at $u_{i}$ are parallel on $\Gamma_{b}$, hence $u_{i}$ is a label of Scharlemann bigon. Since this is true for all $i, \Gamma_{a}$ is kleinian.

Claim 4. $w_{11}+w_{12}=2 n-2$.

Since $\Gamma_{a}$ is kleinian, by Lemma 6.2(4) there is a free involution $\eta$ of $\Gamma_{a}$ mapping $u_{1}$ to $u_{2}$ and $u_{3}$ to $u_{4}$. Thus $\eta$ must map $\hat{e}_{10}$ to $\hat{e}_{7}$ and hence $w_{7}=w_{10}$. We now have $w_{2}+w_{3}+w_{7}=w_{2}+w_{3}+w_{10} \geq 4 n-w_{8}-w_{9} \geq 2 n$. Since $w_{1} \geq 1$, we see that $w_{11}+w_{12}=4 n-\left(2 w_{1}+w_{2}+w_{3}+w_{7}\right) \leq 2 n-2$. The result now follows from Claim 2.

The involution $\eta$ maps $\hat{e}_{11}$ to $\hat{e}_{12}$. Hence Claim 4 implies that $w_{11}=w_{12}=$ $n-1$. Since $\eta$ is label preserving, the label sequence of $\hat{e}_{11} \cup \hat{e}_{12}$ on $\partial u_{3}$ is the same as that on $\partial u_{4}$, so we may assume without loss of generality that the label sequences are as shown in Figure 9.2. One can see that in this case the transition function of $\hat{e}_{11}$ defined in Section 2 is transitive, which implies that all vertices of $\Gamma_{b}$ are parallel, contradicting the assumption. This completes the proof of the lemma.

Proposition 9.7 Suppose $n_{a}=4$ and $\Gamma_{b}$ is non-positive. Then $n_{b} \leq 4$.

Proof. Consider $\hat{\Gamma}_{a}^{+}$. If $\hat{\Gamma}_{a}^{+}$has a small component then by Proposition 8.6 we have $n_{b} \leq 4$. If $\hat{\Gamma}_{a}^{+}$has no small component then the component $G$ containing $u_{1}$ must also contain $u_{3}$, and it is either of type (3), (9) or (10). The result follows from Lemma 9.4 if both components are of type (9) or (10), and from Lemmas 9.5 and 9.6 if at least one component is of type (3).

\section{The case $n_{1}=n_{2}=4$ and $\Gamma_{1}, \Gamma_{2}$ non-positive}

In this section we assume that $n_{1}=n_{2}=4$ and $\Gamma_{a}$ is non-positive for $a=1,2$. We will show that this case cannot happen. Denote by $X_{a}$ the union of $\hat{\Gamma}_{a}^{+}$ and all its disk faces. By Theorem 8.6 $\hat{\Gamma}_{a}^{+}$has no small component, so each component of $\hat{\Gamma}_{a}^{+}$is of type (3), (9) or (10) in Figure 4.2.

Lemma 10.1 Suppose $n_{1}=n_{2}=4$, and both $\Gamma_{1}$ and $\Gamma_{2}$ are non-positive. Then at least one component of $\hat{\Gamma}_{1}^{+}$or $\hat{\Gamma}_{2}^{+}$is of type (3).

Proof. Suppose to the contrary that all components of $\hat{\Gamma}_{1}^{+}$and $\hat{\Gamma}_{2}^{+}$are of type (9) or (10). Then each component of $X_{a}$ is an annulus, hence any vertex $u_{i}$ of $\Gamma_{a}$ is incident to at most 2 families of negative edges. By Lemma 2.3(1) each negative family contains at most 4 edges, so $u_{i}$ is incident to at most 8 negative edges, and hence the number of negative edges is no more than the number of 
positive edges in $\Gamma_{a}$. Since this is true for $a=1,2$ and since a positive edge in one graph is a negative edge in the other, the numbers of positive and negative edges of $\Gamma_{a}$ must be the same, hence each vertex must be incident to exactly 8 positive and 8 negative edges, so each negative family contains exactly 4 edges. Since $\Gamma_{b}$ contains a loop, one of the negative families in $\Gamma_{a}$ contains a co-loop and hence is a set of 4 parallel co-loops, which is a contradiction to the 3-Cycle Lemma 2.14(2).

Lemma 10.2 Suppose $n_{1}=n_{2}=4$, and both $\Gamma_{1}$ and $\Gamma_{1}$ are non-positive. If both components of $\hat{\Gamma}_{a}^{+}$are of type (3), and no component of $\hat{\Gamma}_{b}^{+}$is of type (3), then $\Gamma_{a}$ is kleinian.

Proof. Note that in this case all vertices of $\Gamma_{b}$ are boundary vertices. Let $u_{1}, u_{2}$ be the vertices of valence 2 in $\hat{\Gamma}_{a}^{+}$. By Lemma $6.6(1), u_{1}$ is incident to fewer than 8 positive edges, hence there are three negative edges in $\Gamma_{a}$ incident to $u_{1}$ having the same label $j$ at $u_{1}$. On $\Gamma_{b}$ this implies that the vertex $v_{j}$ is incident to at least three positive edges with label 1 at $v_{j}$; since $v_{j}$ is a boundary vertex, it is incident to at least 9 positive edges. Since a loop at $v_{j}$ must have labels of different parity on its two endpoints, we see that $\operatorname{val}\left(v_{j}, \Gamma_{b}^{+}\right)$is even. If $\operatorname{val}\left(v_{j}, \Gamma_{b}^{+}\right)=12$ then by Lemma 6.4(4) $\Gamma_{a}$ is kleinian. Hence we may assume that $\operatorname{val}\left(v_{j}, \Gamma_{b}^{+}\right)=10$. By Lemma 6.4(1) we may assume that each family of positive edges at $v_{j}$ has at most 3 edges. This implies that $v_{j}$ is incident to 2 or 3 loops. Examining the labels we see that the two outermost loops form a Scharlemann bigon, with 1 as a label. For the same reason, 2 is a label of a Scharlemann bigon in $\Gamma_{b}$. If there is no (12)-Scharlemann bigon then there must be (14)- and (23)-Scharlemann bigon, so $\Gamma_{a}$ is kleinian and we are done. Therefore we may assume that $\Gamma_{b}$ contains a (12)-Scharlemann bigon.

The (12)-Scharlemann bigon and $\hat{\Gamma}_{a}^{+}$cuts $\hat{F}_{a}$ into faces. There is now only one edge class in these faces which connects $u_{1}$ to $u_{4}$, hence by Lemma 2.2(5) $\Gamma_{b}$ contains no (14)-Scharlemann bigon. Similarly there is no (23)-Scharlemann bigon. It follows that all Scharlemann bigons of $\Gamma_{b}$ have label pair (12). In particular, the two outermost loops at $v_{j}$ must form a (12)-Scharlemann bigon.

We have shown above that the vertex $v_{j}$ has 2 or 3 loops. If it has 2 loops then the weights of the positive families at $v_{j}$ are $(2,3,3,2)$, and the middle label of a family of 3 is a label of Scharlemann bigon, which implies that both 3 and 4 are labels of Scharlemann bigons, which is a contradiction. Hence $v_{j}$ has exactly 3 loops $e_{1}, e_{2}, e_{3}$, and 4 non-loop edges divided to 2 non-Scharlemann bigons $e_{4} \cup e_{5}$ and $e_{6} \cup e_{7}$.

As shown above, $e_{1} \cup e_{2}$ is a (12)-Scharlemann bigon, so up to symmetry we may assume that the edges $e_{4}, e_{5}$ have labels 4 and 1 at $v_{j}$. Since these edges do not form a Scharlemann bigon, the labels at their other endpoints must be 3 and 2 respectively, so $e_{5}$ is a (12)-edge, which must be parallel on $\Gamma_{a}$ to one of the two (12)-loops $e_{1}, e_{2}$ because $\Gamma_{a}$ has only two families connecting $u_{1}$ to $u_{2}$. This is a contradiction because by Lemma 2.3(5) a loop and a non-loop edge cannot be parallel on $\Gamma_{a}$. 
Lemma 10.3 Suppose $n_{1}=n_{2}=4$, and both $\Gamma_{1}$ and $\Gamma_{2}$ are non-positive. Then all components of $\hat{\Gamma}_{1}^{+}$and $\hat{\Gamma}_{2}^{+}$are of type (3), and $\hat{\Gamma}_{1}$ and $\hat{\Gamma}_{2}$ are subgraphs of the graph shown in Figure 10.1.

Proof. By Lemma 10.1 we may assume that $\hat{\Gamma}_{a}^{+}$has a component $C$ of type (3). Let $u_{1}$ be the valence 2 vertex in $C$. If $u_{1}$ is incident to at most 4 families of parallel edges then each family contains exactly 4 edges, but since the two positive families are adjacent, this would be a contradiction to Lemma 6.6(1). Therefore $u_{1}$ is incident to at least 5 families of edges. Note that if the other component $C^{\prime}$ of $\hat{\Gamma}_{a}^{+}$is not of type (3), or if it is of type (3) but the loop of $C^{\prime}$ separates $u_{1}$ from the valence 2 vertex $u_{2}$ of $C^{\prime}$ then $u_{1}$ would have only two families of negative edges, which is a contradiction. It follows that $C^{\prime}$ is also of type (3), so the graph $\hat{\Gamma}_{a}$ is a subgraph of that in Figure 10.1.

For the same reason if some component of $\hat{\Gamma}_{b}^{+}$is of type (3) then $\hat{\Gamma}_{b}$ is a subgraph of that in Figure 10.1 and we are done. Therefore we may assume that no component of $\hat{\Gamma}_{b}^{+}$is of type (3). By Lemma $10.2 \Gamma_{a}$ is kleinian.

Consider the edges $\hat{e}_{1}, \ldots, \hat{e}_{6}$ of $\hat{\Gamma}_{a}$ incident to $u_{3}$. See Figure 10.1. Let $p_{i}$ be the weight of $\hat{e}_{i}$. By Lemmas 6.2(2) and 2.3(1), $p_{i}$ is even and at most 4 . Note that $p_{1}, p_{2}, p_{3}$ are non-zero, so by Lemma 6.6(1) we know that $p_{1}+p_{2}$ and $p_{1}+p_{3}$ are between 4 and 6 . If $p_{4}=0$ then $u_{2}$ would have valence 4 in $\hat{\Gamma}_{a}$, which would lead to a contradiction as above. Hence $p_{4}>0$. If $p_{5}+p_{6}=0$ then $\operatorname{val}\left(u_{3}, \hat{\Gamma}_{a}^{+}\right)=5$, so either $p_{1}=4$ or $p_{2}=p_{3}=p_{4}=4$; either case contradicts Lemma 6.6(1). Therefore $p_{5}+p_{6} \geq 2$.

One can now check, from the labeling around the boundary of $u_{3}$, that if $p_{4}=2$ then both labels at the edge endpoints of $\hat{e}_{4}$ at $u_{3}$ are jumping labels at $u_{3}$, and if $p_{4}=4$ then all labels at the endpoints of $\hat{e}_{5} \cup \hat{e}_{6}$ at $u_{3}$ are jumping labels. This is a contradiction because all vertices of $\hat{\Gamma}_{b}^{+}$are boundary vertices and hence by Lemma $6.8 u_{3}$ should have no jumping label.

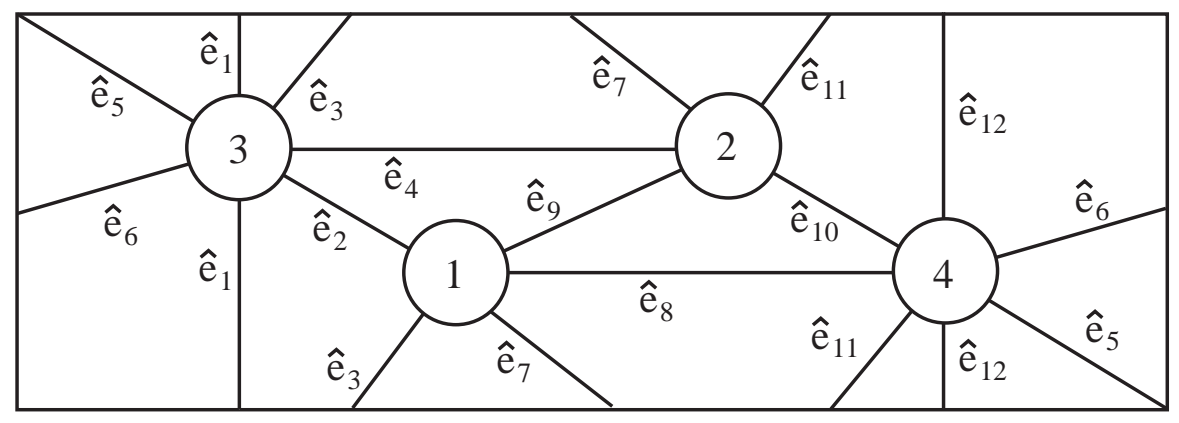

Figure 10.1

By Lemma 10.3 we may assume that both $\hat{\Gamma}_{1}, \hat{\Gamma}_{2}$ are subgraphs of that in Figure 10.1. Label the edges of $\hat{\Gamma}_{a}$ as in Figure 10.1, and let $p_{i}$ be the weight of $\hat{e}_{i}$. Label $\hat{\Gamma}_{b}$ similarly using $\hat{e}_{i}^{\prime}$. 
Lemma $10.4 \Delta=4, p_{2}+p_{3}=6, p_{8}=p_{4}=2$, and $p_{7}=p_{9}=4$. Moreover, edges in $\hat{e}_{4} \cup \hat{e}_{8}$ are co-loops, while those in $\hat{e}_{7} \cup \hat{e}_{9}$ are not.

Proof. If $p_{2}+p_{3}>6$ then one of the $\hat{e}_{2}, \hat{e}_{3}$ contains 4 edges, so by Lemma 6.4(1) $\Gamma_{a}$ is kleinian. By Lemma 6.2(2) the $p_{i}$ are even, so $p_{2}+p_{3}>6$ implies that $p_{2}=p_{3}=4$, which is a contradiction to Lemma 6.6(2). Therefore we have $p_{2}+p_{3} \leq 6$. Since each $p_{i} \leq 4$, by counting edges at $u_{1}$ we have $\Delta=4$, and $p_{7}+p_{8}+p_{9} \geq 10$.

Recall that if a pair of negative edges of $\Gamma_{a}$ incident to $u_{i}$ are parallel in $\Gamma_{b}$ then they form a Scharlemann bigon in $\Gamma_{b}$, hence must have the same label pair in $\Gamma_{b}$, so they are incident to the same pair of vertices in $\Gamma_{a}$. Therefore no edge in $\hat{e}_{7}$ or $\hat{e}_{9}$ is parallel in $\Gamma_{b}$ to an edge in $\hat{e}_{8}$. Since $\hat{\Gamma}_{b}^{+}$has at most 6 edges, we have $\max \left\{p_{7}, p_{9}\right\}+p_{8} \leq 6$. Since $p_{i} \leq 4$, this gives $p_{7}+p_{8}+p_{9} \leq 10$, hence $p_{2}+p_{3}=\Delta n_{b}-\left(p_{7}+p_{8}+p_{9}\right) \geq 6$. Together with the inequalities above, we have $p_{2}+p_{3}=6, p_{7}+p_{8}+p_{9}=10$. Since $\max \left\{p_{7}, p_{9}\right\}+p_{8} \leq 6$ and $p_{i} \leq 4$, this holds only if $p_{8}=2$ and $p_{7}=p_{9}=4$. Similarly $p_{4}=2$.

We have shown that edges in $\hat{e}_{7} \cup \hat{e}_{8}$ belong to distinct families in $\hat{\Gamma}_{b}^{+}$. Since $\hat{\Gamma}_{b}^{+}$has at most 6 edges, $\hat{e}_{7} \cup \hat{e}_{8}$ represent all edges in $\hat{\Gamma}_{b}^{+}$. If some edge in $\hat{e}_{i}$ is a co-loop then all of them are. Therefore the edges in $\hat{e}_{7}$ cannot be co-loops because $\hat{\Gamma}_{b}^{+}$has only two loops. It follows that the edges in $\hat{e}_{8}$ must be co-loops. Similarly, edges of $\hat{e}_{4}$ are co-loops, and those in $\hat{e}_{9}$ are not.

Proposition 10.5 Suppose both $\Gamma_{1}$ and $\Gamma_{2}$ are non-positive, and $n_{a}=4$. Then $n_{b}<4$.

Proof. By Proposition 9.7 we have $n_{b} \leq 4$. Assume to the contrary that $n_{b}=4$. Since the two edges in $\hat{e}_{8}$ are co-loops, they have labels 3,4 at $u_{1}$. Consider the three negative edges $e_{7}, e_{8}, e_{9}$ such that $e_{i} \in \hat{e}_{i}$, and they all have label 3 at $u_{1}$. In $\Gamma_{b}$ these are 1-edges at $v_{3}$. Since $e_{8}$ is a loop, it belongs to $\hat{e}_{1}^{\prime}$. The other two edges are non-loop positive edges on $\Gamma_{b}$, so they belong to $\hat{e}_{2}^{\prime} \cup \hat{e}_{3}^{\prime}$. Applying Lemma 10.4 to $\Gamma_{b}$, we see that $\hat{e}_{2}^{\prime} \cup \hat{e}_{3}^{\prime} \cup \hat{e}_{4}^{\prime}$ contains 8 edges, so the two edges $e_{7}, e_{9}$ are adjacent among the four edges labeled 1 at $v_{3}$ in $\Gamma_{b}$. Since they are not adjacent among the four edges labeled 3 at $u_{1}$ in $\Gamma_{a}$, this is a contradiction to the Jumping Lemma 2.18.

\section{The case $n_{a}=4$, and $\Gamma_{b}$ positive}

In this section we assume that $n_{a}=4$ and $\Gamma_{b}$ is positive. We will determine all the possible graphs for this case. Recall from Lemma 6.4(2) that in this case $\Gamma_{a}$ is kleinian, so the weights of edges of $\hat{\Gamma}_{b}$ are all even.

Lemma 11.1 Suppose $n_{a}=4$ and $\Gamma_{b}$ is positive.

(1) Two families of 4 parallel edges with the same label sequence at a given vertex $v_{j}$ of $\Gamma_{b}$ connect $v_{j}$ to the same vertex $v_{k}$. 
(2) There are at most three families of 4 parallel edges with the same label sequence at any vertex $v_{j}$, and if $n_{b}>2$ then there are at most two such.

(3) if $\Delta=4$ then $\operatorname{val}\left(v_{j}, \hat{\Gamma}_{b}\right) \geq 5$ for all $j$;

(4) if $\Delta=5$ then $\operatorname{val}\left(v_{j}, \hat{\Gamma}_{b}\right) \geq 6$ for all $j$;

(5) two weight 4 edges $\hat{e}_{1}, \hat{e}_{2}$ of $\hat{\Gamma}_{b}$ adjacent at a vertex $v_{j}$ form an essential loop on $\hat{F}_{b}$.

Proof. (1) If there are two families of 4 parallel edges with the same label sequence 1,2,3,4 at $v_{j}$ then by Lemma 6.5 the initial edges $e_{1}, e_{1}^{\prime}$ of the two families are parallel in $\Gamma_{a}$, with the same label $j$ at the vertex $u_{1}$, hence the other endpoints of $e_{1}$ and $e_{1}^{\prime}$ must also have the same label $k$, which implies that in $\Gamma_{b}$ the two families have the same endpoints.

(2) If there were four then the leading edges of the (12) Scharlemann bigons in these families are parallel in $\Gamma_{a}$, so they belong to a family of at least $3 n_{b}+1$ parallel edges connecting $u_{1}$ to $u_{2}$. Since $\hat{\Gamma}_{b}$ has at most $3 n_{b}$ edges by Lemma 2.5 , two of these edges would be parallel on both graphs, which is a contradiction to Lemma 2.2(2).

If $n_{b}>2$ and there are three families of 4 parallel edges with the same label sequence at $v_{j}$ then as above there would be a family of $2 n_{b}+1$ parallel edges in $\Gamma_{a}$, which contradicts Lemma 2.22(3).

(3) and (4) follow immediately from (2).

(5) By (1) these two edges have their other endpoints at the same vertex $v_{k}$, hence form a loop $C=\hat{e}_{1} \cup \hat{e}_{2}$ on $\hat{F}_{b}$. If $C$ is not essential then we can choose $C$ to be an innermost such cycle. $C$ bounds a disk $D$ on $\hat{F}_{b}$, which must contain some vertex because $\hat{\Gamma}_{b}$ is reduced. If some vertex in the interior of $D$ has valence 5 then it is incident to two adjacent weight 4 edges, which would form another inessential loop, contradicting the choice of $C$. Hence all vertices in the interior of $D$ have valence at least 6 . By Lemma 2.9 in this case there should be at least three vertices on $\partial D$, which is a contradiction.

$\hat{F}_{a}$ separates $M\left(r_{a}\right)=M \cup V_{a}$ into the black and white sides $X_{B}$ and $X_{W}$. Since $\Gamma_{b}$ is kleinian (Lemma $6.4(2)$ ), the black side $X_{B}$ is a twisted $I$-bundle over the Klein bottle. A face of $\Gamma_{b}$ is white if it lies in the white region $X_{W}$, otherwise it is black. In the next two lemmas we assume that $\Gamma_{b}$ contains a white bigon and a white 3-gon as in Figure 11.1. 


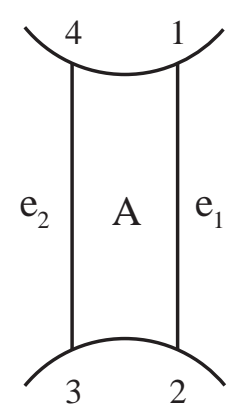

(a)

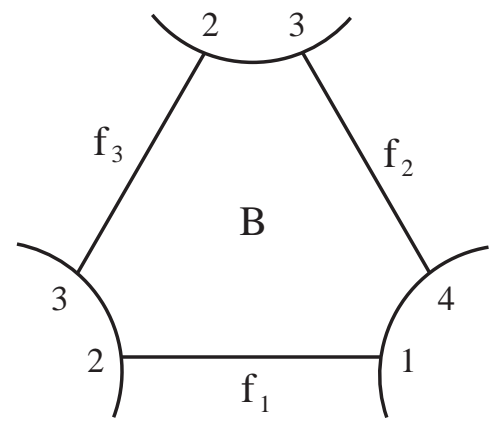

(b)

Figure 11.1

Lemma 11.2 Suppose $n_{a}=4$ and $\Gamma_{b}$ is positive. Suppose $\Gamma_{b}$ contains a white bigon $A$ and a white 3-gon $B$ as in Figure 11.1. Then up to homeomorphism of $\hat{F}_{a}$, the edges of $A$ and $B$ appear on $\hat{F}_{a}$ as shown in Figure 11.2.

Proof. Let $V_{23}$ and $V_{41}$ be the components of $V_{a} \cap X_{W}$ that run between $u_{2}, u_{3}$ and $u_{4}, u_{1}$, respectively. Let $Y$ be a regular neighborhood of $\hat{F}_{a} \cup V_{23} \cup V_{41} \cup A \cup B$. Then $\partial Y=\hat{F}_{a} \cup T$, where $T$ is a torus in $M$, and hence either $X_{W}=Y$, or $X_{W}$ is the union of $Y$ and a solid torus along $T$.

Take a regular neighborhood $D$ of $e_{1} \cup e_{2} \cup f_{3}$ as "base point" for $\pi_{1}\left(\hat{F}_{a}\right) \cong$ $\mathbb{Z} \times \mathbb{Z}$ and for $\pi_{1}\left(X_{W}\right)$. (See Figure 11.2). The cores of the 1-handles $V_{23}$ and $V_{41}$ represents elements $x, y$ respectively of $\pi_{1}\left(X_{W}\right)$, and $\pi_{1}\left(X_{W}\right)$ is generated by $\Pi=\pi_{1}\left(\hat{F}_{a}\right)$ together with $x$ and $y$. Note that since $\hat{F}_{a}$ is essential in $M\left(r_{a}\right)$, $\Pi$ is a proper subgroup of $\pi_{1}\left(X_{W}\right)$.

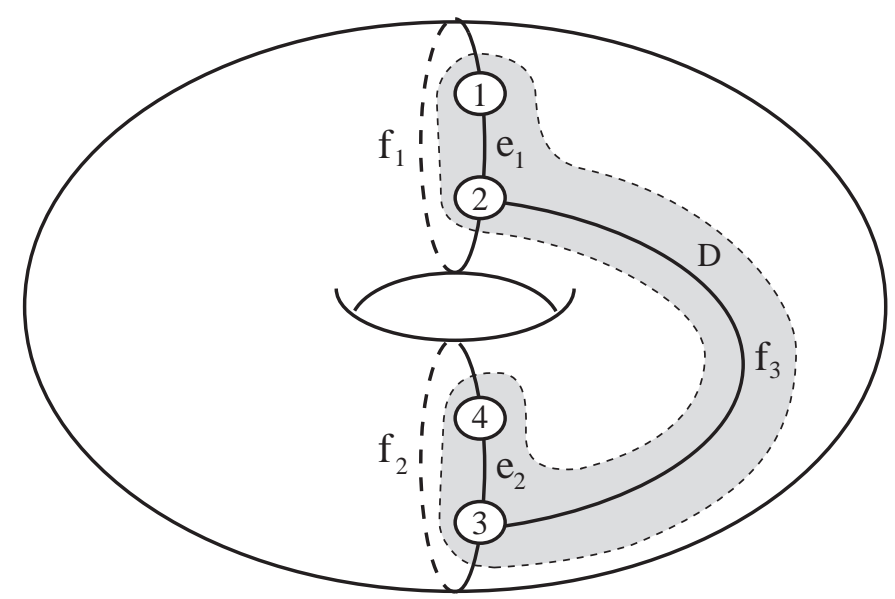

Figure 11.2 
The bigon $A$ gives the relation $x y=1$, so the 23 -corners and the 41 -corner of $\partial B$ represent $x$ and $x^{-1}$ respectively. The 32-edge on $\partial B$ lies in $D$ and hence represents 1 . If one of the other two edges $f_{1}, f_{2}$ of $B$ represents $1 \in \pi_{1}\left(\hat{F}_{a}\right)$, then $B$ gives a relation of the form $x=\gamma$ for some $\gamma \in \pi_{1}\left(\hat{F}_{a}\right)$, hence $\pi_{1}\left(X_{W}\right)=\Pi$, a contradiction. Therefore the edge $f_{1}$ is as shown in Figure 11.2, and represents a nontrivial element $\alpha \in \pi_{1}\left(\hat{F}_{a}\right)$, when oriented from $u_{1}$ to $u_{2}$. Similarly, the edge $f_{2}$ represents a non-trivial element $\gamma$, say, of $\pi_{1}\left(\hat{F}_{a}\right)$, when oriented from $u_{3}$ to $u_{4}$. Since $e_{1} \cup f_{1}$ and $e_{2} \cup f_{2}$ are disjoint, we must have $\gamma=\alpha$ or $\alpha^{-1}$. The union $V_{23} \cup V_{41} \cup N(A)$ is a single 1-handle attached to $\hat{F}_{a}$, and $\partial B$ is a simple closed curve on $\hat{F}_{a} \cup \partial H$. One can see that $\alpha x^{2} \alpha^{-1} x^{-1}$ cannot be realized by a simple closed curve. It follows that $\gamma=\alpha$, so $f_{2}$ appears on $\hat{F}_{a}$ as in Figure 11.2 .

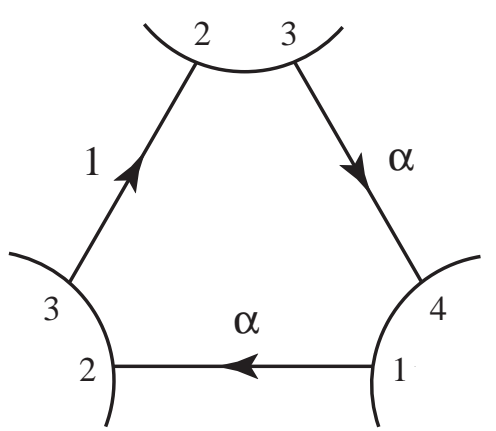

(a)

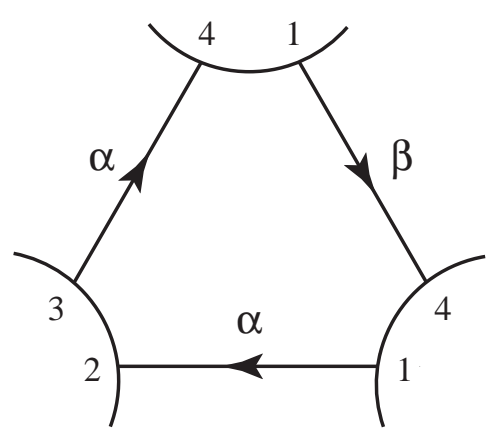

(b)

Figure 11.3

If we orient an edge $e$ of $\Gamma_{b}$ then the corresponding oriented edge of $\Gamma_{a}$ represents an element $\gamma$ of $\pi_{1}\left(\hat{F}_{a}\right)$, and we will label the edge $e$ with $\gamma$. Thus the edge labels of the bigon $A$ are both 1 , and the edge labels of the 3-gon $B$ are as in Figure 11.3(a). Note that any 12-edge, oriented from 1 to 2, or any 34 -edge, oriented from 3 to 4 , has label 1 or $\alpha$. Also $\pi_{1}\left(\hat{F}_{a}\right)$ has a basis $\{\alpha, \beta\}$, where $\beta$ is represented by an arc joining $u_{1}$ and $u_{4}$, disjoint from $\partial A$ and $\partial B$, as shown in Figure 11.4. 


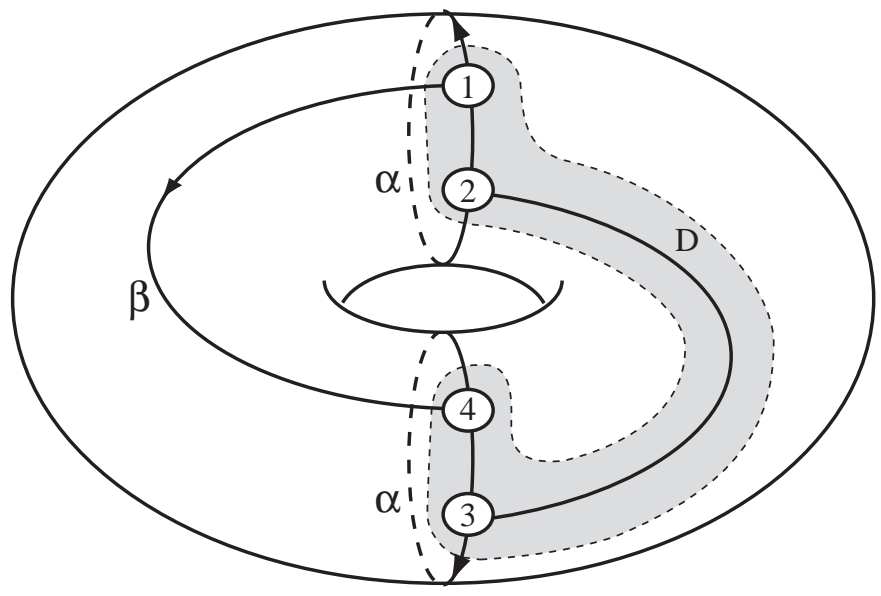

Figure 11.4

Lemma 11.3 Suppose $n_{a}=4$ and $\Gamma_{b}$ is positive. Suppose $\Gamma_{b}$ contains a white bigon $A$ and a white 3-gon $B$ as in Figure 11.1.

(1) All edges of white bigons have label 1.

(2) $\beta$ can be chosen so that any 3-gon must have edge labels as shown in Figure 11.3(a) or (b).

Proof. (1) Since the edges $e_{1} \cup f_{1}$ and $e_{2} \cup f_{2}$ on $\partial A$ and $\partial B$ form two parallel essential circles on $\hat{F}_{a}$, any 12 -edge of $\Gamma_{b}$ must be parallel to either $e_{1}$ or $f_{1}$, and any 34-edge of $\Gamma_{b}$ must be parallel to either $e_{2}$ or $f_{2}$.

Let $A^{\prime}$ be another white bigon. Applying Lemma 11.2 to $A^{\prime}$ and $B$ gives that the 12-edge of $A^{\prime}$ and the 12-edge $f_{1}$ of $B$ are not parallel, and similarly the 34-edge of $A^{\prime}$ and the 34-edge $f_{2}$ of $B$ are not parallel. Hence both edges of $A^{\prime}$ are labeled 1 since they must be parallel to $e_{1}$ and $e_{2}$, respectively.

(2) Since $\Gamma_{b}$ has no extended Scharlemann cycle, each triangle face $B^{\prime}$ has either one or two 23-corners. If $B^{\prime}$ has two 23-corners then applying Lemma 11.2 to $A$ and $B^{\prime}$ shows that the 12-edge of $B^{\prime}$ is not parallel to $e_{1}$, so it must be parallel to $f_{1}$ and hence is labeled $\alpha$, as in Figure 11.3(a). Similarly the 34-edge of $B^{\prime}$ is also labeled $\alpha$. If the 32-edge of $B^{\prime}$ is labeled $\gamma$ then $B$ and $B^{\prime}$ together give the relation $\gamma=1$, so $\gamma=1$, as in Figure 11.3(a).

If $B^{\prime}$ has only one 23 -corner, let $f_{1}^{\prime}, f_{2}^{\prime}, f_{3}^{\prime}$ be the 12-, 34- and 14-edges of $B^{\prime}$, respectively. Applying Lemma 11.2 to $A$ and $B^{\prime}$ gives that $f_{1}^{\prime}$ is not parallel to $e_{1}$, so by the above it must be parallel to $f_{1}$ and hence is labeled $\alpha$. Similarly $f_{2}^{\prime}$ is parallel to $f_{2}$ and is also labeled $\alpha$. The two loops $e_{1} \cup f_{1}$ and $e_{2} \cup f_{2}$ cut $\hat{F}_{a}$ into two annuli $A_{L}$ and $A_{R}$, where $A_{R}$ contains $f_{3}$; see Figure 11.4. If the 14-edge $f_{3}^{\prime}$ of $B^{\prime}$ lies in $A_{R}$ then it is labeled $\alpha$, and $B^{\prime}$ gives the relation $x^{-1} \alpha x \alpha x^{-1} \alpha=1$. It is easy to see that, together with the relation $x^{2} \alpha x^{-1} \alpha=1$ coming from $B$, this implies $a=x^{2}$, so $x^{5}=1$, and hence $\alpha^{5}=1$, which is a contradiction to the fact that $\alpha^{5}$ is a nontrivial element in $\pi_{1}\left(\hat{F}_{a}\right)$ and hence is 
nontrivial in $\pi_{1}\left(X_{W}\right)$. Therefore $f_{3}^{\prime}$ lies in $A_{L}$, as shown in Figure 11.4. Let $\beta$ be the corresponding element of $\pi_{1}\left(\hat{F}_{a}\right)$; then the edge labels of $B^{\prime}$ are as in Figure $11.3(\mathrm{~b})$. If $B^{\prime \prime}$ is any other 3 -gon with one 23 -corner then the argument above in the case of 3 -gons with two 23 -corners, using $A$ and the present $B^{\prime}$, shows that the edge labels of $B^{\prime \prime}$ are also as shown in Figure 11.3(b).

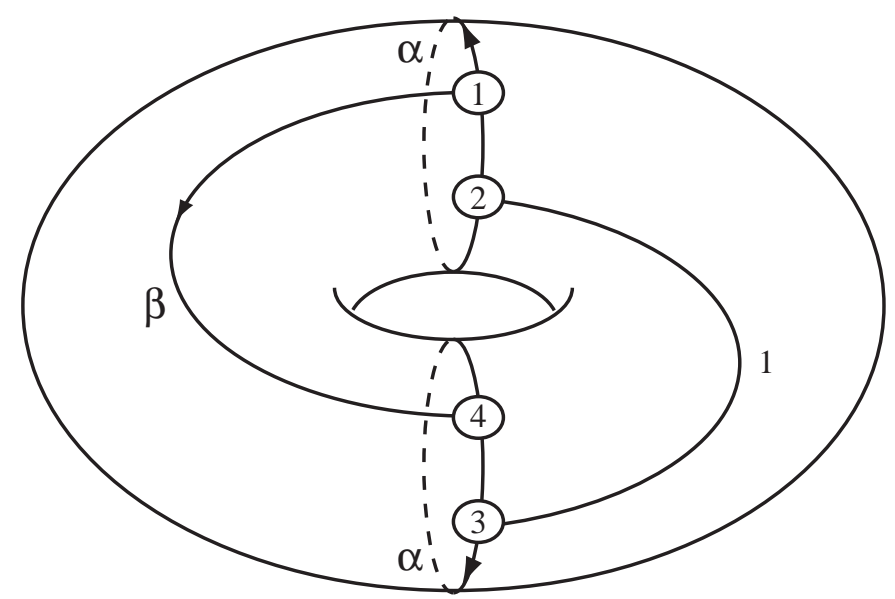

Figure 11.5

Corollary 11.4 Suppose $n_{a}=4$ and $\Gamma_{b}$ is positive.

(1) Let $G$ be the subgraph of $\Gamma_{a}$ consisting of edges of white bigons and white 3-gons on $\Gamma_{b}$. Then the reduced graph $\hat{G}$ is a subgraph of that in Figure 11.5.

(2) If $\Gamma_{b}$ contains a white bigon then it cannot contain a (black) Scharlemann bigon which is flanked on each side by a (white) 3-gon.

(3) If $\Gamma_{b}$ contains a white bigon then it cannot contain three 3-gons occurring as consecutive white faces at a vertex.

Proof. (1) This follows immediately from Lemma 11.3.

(2) The edges of a (black) Scharlemann bigon are either (12)- or (34)-edges, so by Lemma 11.3(2) both edges of the Scharlemann bigon are labeled $\alpha$ and hence are parallel on $\Gamma_{a}$, contradicting Lemma 2.2(2).

(3) By (2) the two black bigons between the white 3 -gons are (12,34)-bigons, as shown in Figure 11.6. But then the middle 3-gon would be a white Scharlemann cycle, contradicting Lemma 6.2(3). 


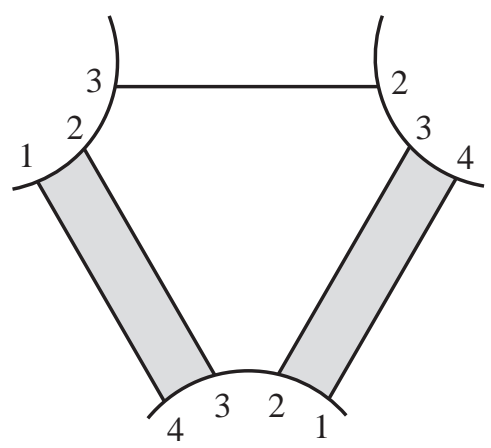

Figure 11.6

Let $G$ be a reduced graph on a torus $T$ with disk faces. One can endow $T$ with a singular Euclidean structure by letting each edge have length 1 and each $n$-gon face a regular Euclidean $n$-gon. The cone angle $\theta(v)$ at a vertex $v$ of $G$ is the sum of the angles of the corners incident to $v$. Such a structure is hyperbolic if $\theta(v) \geq 2 \pi$ for all $v$, and $\theta(v)>2 \pi$ for some $v$. The following lemma says that no singular Euclidean structure on $T$ is hyperbolic.

Lemma 11.5 Let $G$ and $\theta(v)$ be defined as above. Then either $\theta(v)<2 \pi$ for some $v$, or $\theta(v)=2 \pi$ for all $v$.

Proof. Denote by $V, E, F$ the numbers of vertices, edges and faces of $G$, respectively. Let $\theta(c)$ be the angle at a corner $c$ of the graph. If $\sigma$ is a face of $G$, denote by $|\sigma|$ the number of edges of $\sigma$. Since $\sigma$ is a regular $|\sigma|$-gon, for each corner $c \in \sigma$ we have $\theta(c)=\pi(1-2 /|\sigma|)$. In the following, the first sum is over all vertices $v$ of $G$, and the second is over all corners $c$. Grouping corners by faces $\sigma$, we get

$$
\begin{gathered}
\sum_{v} \theta(v) \quad \sum_{c} \theta(c)=\sum_{\sigma} \sum_{c \in \sigma} \theta(c)=\sum_{\sigma} \sum_{c \in \sigma} \pi\left(1-\frac{2}{|\sigma|}\right) \\
=\pi\left(\sum_{\sigma}|\sigma|-\sum_{\sigma} \sum_{c \in \sigma} \frac{2}{|\sigma|}\right)=\pi(2 E-2 F)=2 \pi(E-F)=2 \pi V .
\end{gathered}
$$

Therefore $\sum_{v}(2 \pi-\theta(v))=0$, and the result follows.

Lemma 11.6 Let $G$ be a reduced graph on a torus $T$ such that val $(v) \geq 5$ for all $v$. Then either

(1) there exists a vertex of valence 5 with at least four 3-gons incident; or

(2) there exists a vertex of valence 6 and all vertices of valence 6 have all incident faces 3 gons; or

(3) all faces of $G$ are 3-gons or 4-gons, and every vertex has valence 5 and has exactly three 3-gons incident. 
Proof. We have $\theta(v)>2 \pi$ if $\operatorname{val}(v)>6, \theta(v) \geq 6 \times \pi / 3=2 \pi$ if $\operatorname{val}(v)=6$. Assuming (1) is not true, then we also have

$$
\theta(v) \geq 3 \times \frac{\pi}{3}+2 \times \frac{\pi}{4}=2 \pi
$$

if $\operatorname{val}(v)=5$. Thus there is no vertex with cone angle $\theta(v)<2 \pi$, so by Lemma 11.5 we see that $\theta(v)=2 \pi$ for all $v \in G$, hence there is no vertex of valence more than 6 , all faces incident to vertices of valence 6 are 3 -gons, and exactly 3 faces incident to a vertex of valence 5 are 3 -gons and the other two are 4-gons. Therefore either (2) or (3) holds.

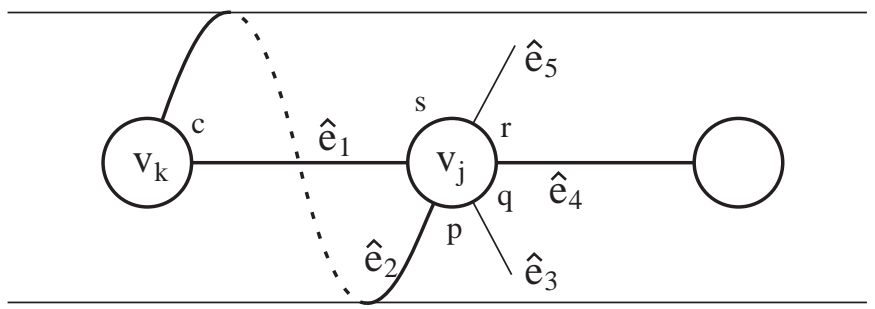

Figure 11.7

Lemma 11.7 Suppose $n_{a}=4, n_{b}>2$, and $\Gamma_{b}$ is positive. Then no vertex $v_{j}$ of $\hat{\Gamma}_{b}$ with $\operatorname{val}\left(v_{j}\right)=5$ has four corners belonging to 3-gons.

Proof. Let $v_{j}$ be a vertex of $\hat{\Gamma}_{b}$ with $\operatorname{val}\left(v_{j}\right)=5$. By Lemma 11.1(4) we must have $\Delta=4$. The weights of the edges at $v_{j}$ are $4,4,4,2,2$. By Lemma 11.1(2) the three weight 4 edges are not consecutive, hence the order around $v_{j}$ is $(4,4,2,4,2)$. Label these edges by $\hat{e}_{1}, \ldots, \hat{e}_{5}$, respectively, where $\hat{e}_{3}, \hat{e}_{5}$ have weight 2. By Lemma 11.1(5) the two edges $\hat{e}_{1}, \hat{e}_{2}$ form an essential cycle on the torus $\hat{F}_{b}$, hence the graph looks like that in Figure 11.7 .

Let $c$ be the corner at $v_{k}$ between these two edges $\hat{e}_{1}, \hat{e}_{2}$, as shown in Figure 11.7. We claim that $c$ contains no other edge endpoint. Let $e$ and $e^{\prime}$ be the edges in $\hat{e}_{1}$ and $\hat{e}_{2}$ with label 1 at $v_{j}$. Let $P, Q$ be the endpoints of $e, e^{\prime}$ at $v_{j}$, and let $R, S$ be the endpoints of $e, e^{\prime}$ at $v_{k}$, respectively. By Lemma 6.5 these edges are parallel on $\Gamma_{a}$, so they connect the same pair of vertices $u_{1}, u_{r}$ for some $r$. On $\Gamma_{b}$ this means that $P, Q$ have the same label 1 , and $R, S$ have the same label $r$.

Since $e, e^{\prime}$ are parallel negative edges on $\Gamma_{a}$, we have $d_{u_{1}}(P, Q)=d_{u_{r}}(R, S)$, therefore the four points $P, Q, R, S$ satisfy the assumptions of Lemma 2.16(1), hence by the lemma we have $d_{v_{j}}(P, Q)=d_{v_{k}}(R, S)$. Without loss of generality assume that the orientations on $\partial v_{j}, \partial v_{k}$ are counterclockwise on Figure 11.7. Then one can see that $d_{v_{j}}(P, Q)=4$, hence $d_{v_{k}}(R, S)=4$, which implies that there are only 3 edge endpoints from the endpoint of $e$ to that of $e^{\prime}$ on $\partial v_{k}$, so there is no edge endpoint in the corner $c$ in Figure 11.7. This proves the claim. 
Label the corners at $v_{j}$ as shown in Figure 11.7. The above implies that the corner $p$ and $s$ belong to the same face $\sigma$, so if $v_{j}$ is incident to at least four 3 -gons then $\sigma$ must be a 3 -gon, hence $\hat{e}_{3}=\hat{e}_{5}$ is a loop. Now the corners $q$ and $r$ belong to the same face $\sigma^{\prime}$, which cannot be a 3-gon, hence the result follows.

Lemma 11.8 Suppose $n_{a}=4, n_{b}>2$, and $\Gamma_{b}$ is positive. Suppose $\Delta=4$.

(1) All faces of $\hat{\Gamma}_{b}$ are 3-gons or 4-gons, every vertex has valence 5 and has exactly three 3-gons incident, and the weight sequence of the edges incident to the vertex is $(4,4,2,4,2)$. In particular, $n_{b}$ is even.

(2) The two weight 2 edges at any vertex form a loop, which is incident to a 3-gon whose other two edges are of weight 4.

(3) Each edge in a weight 2 family of $\Gamma_{b}$ has label pair (23) or (14).

Proof. (1) By Lemma 11.6 and Lemma 11.1(3) and (4), $\hat{\Gamma}_{b}$ is one of the three types stated there. Lemma 11.7 shows that $\hat{\Gamma}_{b}$ cannot be of type (1). If $\hat{\Gamma}_{b}$ has a vertex of valence 6 then the weights are $4,4,2,2,2,2$, hence there are two consecutive edges of weight 2. By Corollary 11.4(3) the three faces incident to these two edges cannot all be 3 -gons, hence $\hat{\Gamma}_{b}$ cannot be of type (2) in Lemma 11.6. It follows that $\hat{\Gamma}_{b}$ is of type (3) in Lemma 11.6, so the weights of the edges at every vertex of $\hat{\Gamma}_{b}$ are $4,4,4,2,2$. Thus the number of weight 4 edge endpoints in $\hat{\Gamma}_{b}$ is $3 n_{b}$, which must be even, hence $n_{b}$ is even. By Lemma 11.1(2) the three weight 4 edges cannot all have the same label sequence, hence the weight sequence is $(4,4,2,4,2)$ at each vertex.

(2) By Lemma 11.1(5) the two adjacent weight 4 edges at $v_{j}$ connects $v_{j}$ to a vertex $v_{k}$ and form an essential loop on $\hat{F}_{b}$. The other weight 4 edge at $v_{j}$ connect to some vertex $v_{r}$, whose two other weight 4 edges connect to another vertex $v_{s}$ and form an essential loop. These five weight 4 edges cut off a 6 -gon containing the four weight 2 edges at $v_{j}$ and $v_{r}$. The 6 -gon cannot contain any vertex in its interior because each vertex is incident to two weight 4 edges forming an essential cycle on $\hat{F}_{b}$. Therefore the four weight 2 edges at $v_{j}$ and $v_{r}$ form two loops.

(3) The loop $\hat{e}$ of $\hat{\Gamma}_{b}$ at $v_{j}$ cuts off a 3 -gon in the 6 -gon above. Let $e_{1}$ be the edge of $\hat{e}$ which is on the boundary of a 3 -gon face $\sigma$ of $\Gamma_{b}$. Then the other two edges of $\sigma$ belong to families of 4 edges and hence must have label pair (12) and (34) respectively, so the labels on $\partial \sigma$ are as shown in Figure 11.3, and $e_{1}$ has label pair (23) or (14). Since the other loop edge of $\hat{e}$ is parallel to $e_{1}$, it has label pair (14) or (23), respectively. 

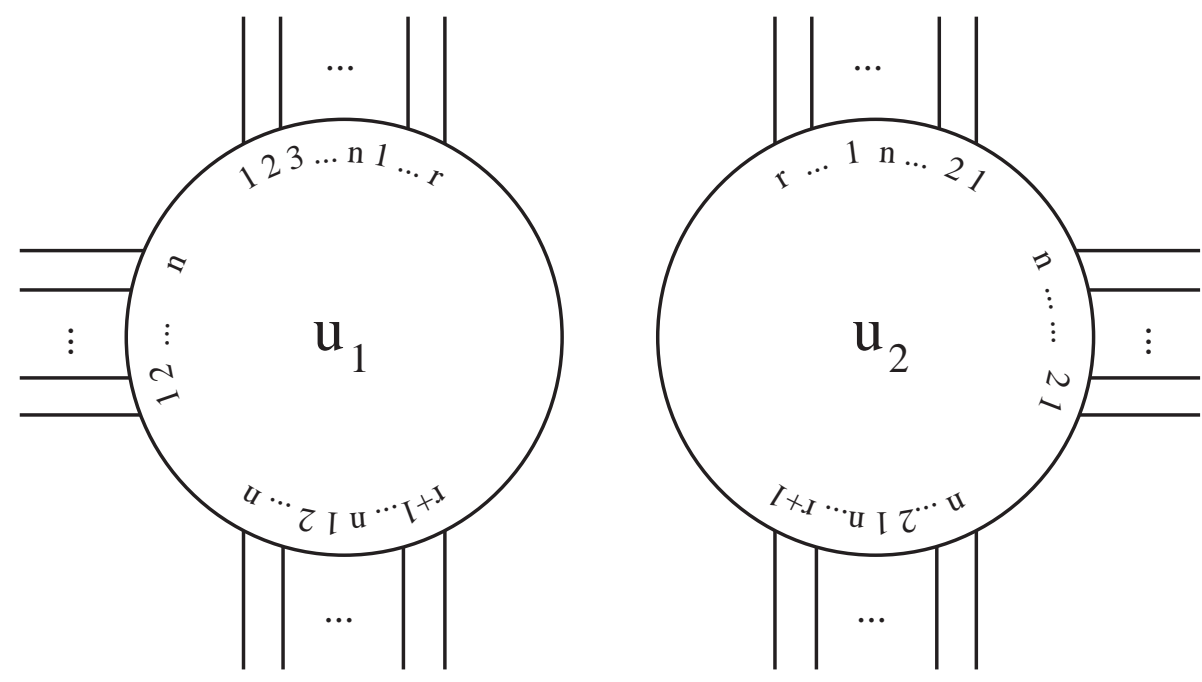

Figure 11.8

Proposition 11.9 Suppose $n_{a}=4$ and $\Gamma_{b}$ is positive.

(1) If $\Delta=4$ then $n_{b}=2$, and the graphs are as shown in Figure 11.9.

(2) If $\Delta=5$ then $n_{b}=1$, and the graphs are as shown in Figure 11.10.

Proof. (1) Put $n=n_{b}$. If $n=1$ then the assumption $\Delta=4$ implies that the weights of the edges of $\hat{\Gamma}_{b}$ are either $(4,4,0)$ or $(4,2,2)$. In either case a family of four edges form an extended Scharlemann cycle, which is impossible.

Now assume $n>2$. Let $C=\hat{e}_{1} \cup \hat{e}_{2}$ be the two edges in $\hat{\Gamma}_{a}$ connecting $u_{1}$ to $u_{2}$. Since $\hat{F}_{a}$ contains both (12)- and (34)-Scharlemann cocycles, any edge of $\Gamma_{b}$ with label pair (12) is parallel to an edge of $C$ on $\Gamma_{a}$. By Lemma 11.8(1) each vertex of $\hat{\Gamma}_{b}$ has valence 5 and hence is incident to 3 weight 4 edges, so $\hat{\Gamma}_{b}$ has $3 n / 2$ weight 4 edges. Each weight 4 edge contributes 2 edges to $C$, one for each $\hat{e}_{i}$, and by Lemma 11.8(3) no weight 2 edge of $\hat{\Gamma}_{b}$ contributes to $C$. Thus each $\hat{e}_{i}$ represents exactly $3 n / 2$ edges, and each label $j$ appears exactly three times among the edge endpoints of $\hat{e}_{1} \cup \hat{e}_{2}$ at $u_{1}$. Hence if the edge endpoints of $\hat{e}_{1}$ at $u_{1}$ are labeled $1, \ldots, n, 1, \ldots, r$, then the labels of those in $\hat{e}_{2}$ must be $r+1, \ldots, n, 1, \ldots, n$, where $r=n / 2$. It follows that the $n$ edge endpoints at $u_{1}$ that do not belong to $C$ must be on one side of $C$, so up to relabeling we may assume that the labels at $u_{1}$ are as shown in Figure 11.8(a). Let $\varphi$ be the involution on $\hat{F}_{a}$ given by Lemma $6.2(4)$. Then $\varphi$ maps $\hat{e}_{1}$ to $\hat{e}_{2}$ and is label preserving, so the labels at $u_{2}$ must be as shown in Figure 11.8(b). Now the transition function of $\hat{e}_{1}$ maps 1 to $r+1$, which has period 2. Since $n>2$, this function is not transitive, contradicting Lemma 2.3(1).

We have shown that $n=2$. The graph $\hat{\Gamma}_{b}$ is now a subgraph of that in Figure 13.1, with vertices labeled $v_{1}, v_{2}$ instead of $u_{1}, u_{2}$. Let $w_{i}$ be the weight of $\hat{e}_{i}$. By Lemma 6.4(2) $\Gamma_{a}$ is kleinian, and by Lemma 6.2(2) the $w_{i}$ are all even. 
By Lemma 11.1(3) we have $w_{5}>0$. If $w_{5}=4$ then $\hat{e}_{5}$ containing no extended Scharlemann bigon implies that either $w_{1}+w_{2}=6$ or $w_{3}+w_{4}=6$, so $w_{i}=4$ for some $i \leq 4$, in which case $\hat{e}_{i}$ contains a (12)-Scharlemann bigon, whose edges, by the above, must be parallel in $\Gamma_{a}$ to the (12)-edges of $\hat{e}_{5}$, which is a contradiction to Lemma 2.3(5). Therefore we must have $w_{5}=2$. For the same reason, the two loops in $\hat{e}_{5}$ cannot be a Scharlemann bigon, so we must have $w_{1}=w_{2}=4$ and $w_{3}+w_{4}=4$ up to symmetry. Let $e_{1} \cup \ldots \cup e_{4}$ and $e_{1}^{\prime} \cup \ldots \cup e_{4}^{\prime}$ be the edges in $\hat{e}_{1}, \hat{e}_{2}$ respectively, such that $e_{i}, e_{i}^{\prime}$ have label $i$ at $v_{1}$. By Lemma $6.5 e_{i}, e_{i}^{\prime}$ are parallel on $\Gamma_{a}$, with the same label 1 at $u_{i}$. Therefore there is another edge between them, which must belong to $\hat{e}_{3} \cup \hat{e}_{4}$. If $w_{3}=w_{4}=2$ then one can check that these edges would have label pairs (14) and (23), which is a contradiction. Therefore we may assume $w_{3}=4$ and $w_{4}=0$. The graph $\Gamma_{b}$ is now as shown in Figure 11.9(b).

As shown above, there are 12 edges on $\Gamma_{b}$ with label pair (12) or (34), divided into 4 families of 3 edges each on $\Gamma_{a}$. Label the edges of $\Gamma_{b}$ as in the figure. Up to symmetry we may assume the edge $A$ is as shown in Figure 11.9(a). Since $\Delta=4$, we may assume that the jumping number $J=1$. The 1-edges around $v_{1}$ appear in the order $A, E, G, P$. By Lemma $6.5 G, P$ are parallel on $\Gamma_{a}$. This determines the position of these edges as well as the orientation of $u_{1}$. The 2-edges at $v_{2}$ appear in the order $E, R, G, P$, and $E$ on $\Gamma_{a}$ has already been determined above, hence the position of $R, G, P$ must appear around $\partial u_{2}$ are shown in the figure. Other edges on $\Gamma_{a}$ can be determined similarly. 


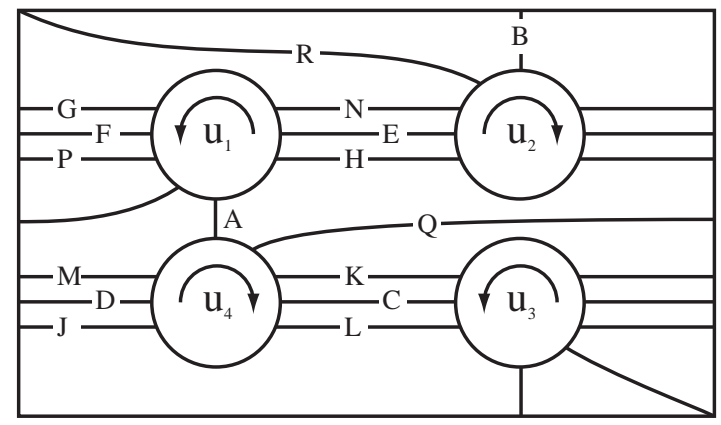

(a)

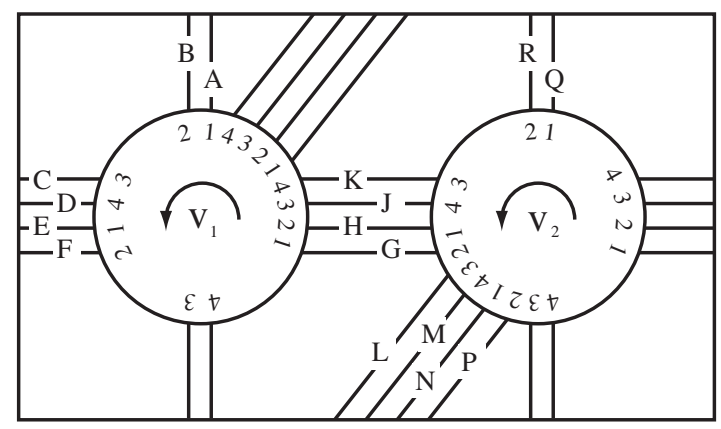

(b)

Figure 11.9

(2) The proof for $\Delta=5$ is similar but simpler. In this case by Lemma 11.1(4) each vertex $v_{j}$ of $\hat{\Gamma}_{b}$ has valence 6 , and the edges at $v_{j}$ have weights $4,4,4,4,2,2$. Thus all white faces are bigons and 3 -gons, so by Corollary 11.4(1) $\hat{\Gamma}_{a}$ is a subgraph of that in Figure 11.5. By Lemma 11.1(2) the two weight 2 edges at any vertex are non-adjacent, thus any edge $e$ in a weight 2 family is on the boundary of a 3-gon whose other two edges have label pairs (12) and (34), so the label pair of $e$ must be (14) or (23) and hence $e$ is not a vertical edge in Figure 11.5. On the other hand, each weight 4 edge of $\hat{\Gamma}_{b}$ contributes one edge to each vertical family in Figure 11.5, hence each vertical edge has weight exactly $2 n_{b}$. As above, one can show that the transition function defined by a family of vertical edges is the identity function, which by Lemma 2.3(1) implies that $n_{b}=1$, so the graph $\Gamma_{b}$ must be as shown in Figure 11.10(b). By the above discussion, $\Gamma_{a}$ is as shown in Figure 11.10(a). Label edges as in Figure 11.10(b). By Lemma 6.5 the edges $A, H$ are parallel on $\Gamma_{b}$, hence we may assume the jumping number $J=1$. One can now easily determine the labels of the edges of $\Gamma_{a}$. 


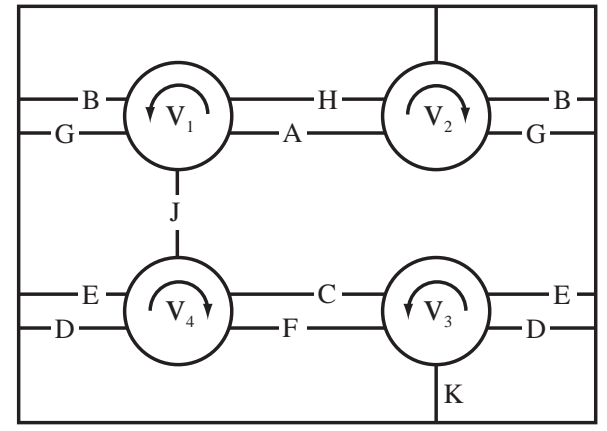

(a)

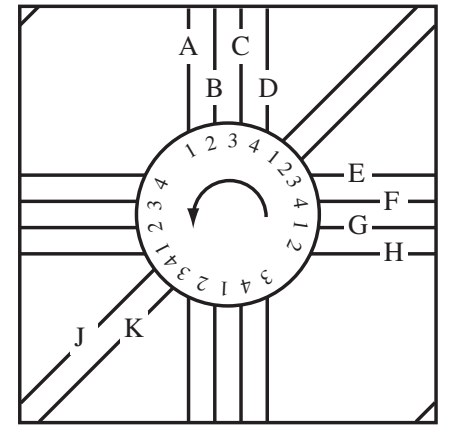

(b)

Figure 11.10

Proposition 11.10 Suppose $n_{a} \leq n_{b}$. Then $n_{a} \leq 2$.

Proof. By Proposition 5.11 we have $n_{a} \leq 4$. Assume $n_{a}=4 \leq n_{b}$. Then by Proposition 11.9, $\Gamma_{b}$ is non-positive. Therefore, by Proposition 9.7, $n_{a}=$ $n_{b}=4$, and, by Proposition 11.9 again, $\Gamma_{a}$ is also non-positive, contradicting Proposition 10.5.

Suppose $n_{a}=3$. Then $\hat{F}_{a}$ is non-separating. If $\Gamma_{a}$ is non-positive then some vertex $u_{1}$, say, has different sign to the other two vertices $u_{2}, u_{3}$. One of the vertices has valence at most two in $\hat{\Gamma}_{a}^{+}$, so it is incident to at most $2 n_{b}$ positive edges, and hence at least $2 n_{b}$ negative edges. By Lemma 2.8 this implies that $\Gamma_{b}$ has a Scharlemann cycle, so by Lemma 2.2(4) $\hat{F}_{a}$ is separating, which is a contradiction. If $\Gamma_{a}$ is positive then by Lemma 3.1 we have $n_{b} \leq 4$. By Lemma $2.23 n_{1}, n_{2}$ cannot both be odd, hence $n_{a}=3$ implies that $n_{b}$ is even, so we must have $n_{b}=4$. Now applying Proposition 11.9 with $n_{a}, n_{b}$ reversed, we get $n_{a} \leq 2$, which is a contradiction.

\section{The case $n_{a}=2, n_{b} \geq 3$, and $\Gamma_{b}$ positive}

The next few sections deal with the case that $n_{a}=2$ and $n_{b} \geq 3$. The main result of this part is Proposition 16.8, which shows that there are only a few possibilities for the graphs $\Gamma_{a}, \Gamma_{b}$.

Throughout this section we will assume that $n_{a}=2, n_{b} \geq 3$ and $\Gamma_{b}$ is positive. We will show that this case is impossible. To simplify notation, denote $n_{b}$ by $n$. Note that $\hat{\Gamma}_{a}$ has at most four edges $\hat{e}_{1}, \ldots, \hat{e}_{4}$, all connecting $u_{1}$ to $u_{2}$. We will always assume that the first edge of $\hat{e}_{1}$ has label 1 at $u_{1}$. Write $\Gamma_{a}=\left(a_{1}, a_{2}, a_{3}, a_{4}\right)$, where $a_{i}$ is the weight of $\hat{e}_{i}$. Let $s_{i}=s\left(\hat{e}_{i}\right)$ be the transition number of $\hat{e}_{i}$ from $u_{1}$ to $u_{2}$. In the following lemma the subscripts are mod 4 integers. 
Lemma 12.1 (1) $s_{i+1} \equiv s_{i}-a_{i}-a_{i+1} \bmod n$;

(2) $s_{i+2} \equiv s_{i}-a_{i+1}+a_{i+3} \bmod n$. In particular, $s_{i} \equiv s_{i+2}$ if and only if $a_{i+1} \equiv a_{i+3} \bmod n$.

Proof. (1) Orient edges from $u_{1}$ to $u_{2}$, and denote by $e(h), e(t)$ the head and tail of an edge $e$. Let $e, e^{\prime}$ be the first edge of $\hat{e}_{i}, \hat{e}_{i+1}$, respectively. Let $x$ be the label of $e(t)$, and $y$ the label of $e^{\prime}(h)$. Then traveling from $e(t)$ to $e^{\prime}(t)$ on $\partial u_{1}$ then to $e^{\prime}(h)$ through $e^{\prime}$ gives $y \equiv x+a_{i}+s_{i+1} \bmod n$, while traveling through $e$ to $e(h)$ then along $\partial u_{2}$ to $e^{\prime}(h)$ gives $y \equiv x+s_{i}-a_{i+1} \bmod n$. Hence $s_{i+1} \equiv s_{i}-a_{i}-a_{i+1} \bmod n$.

(2) Applying (1) twice gives $s_{i+2} \equiv s_{i}-a_{i}-a_{i+1}-a_{i+1}-a_{i+2} \equiv s_{i}-a_{i+1}+$ $a_{i+3} \bmod n$.

Lemma 12.2 Let $e, e^{\prime}$ be edges of $\Gamma_{b}$ joining a pair of distinct vertices, such that $e \cup e^{\prime}$ is null-homotopic in $\hat{F}_{b}$. If e belongs to a family of at least $n$ parallel edges in $\Gamma_{a}$ then e and $e^{\prime}$ are parallel on $\Gamma_{b}$.

Proof. Let $D$ be the disk in $\hat{F}_{b}$ bounded by $e \cup e^{\prime}$. The family of $n$ parallel edges of $\Gamma_{a}$ containing $e$ gives a set of essential loops on $\hat{F}_{b}$, corresponding to the orbits of the associated permutation. It follows that $D$ contains no vertices in its interior, and hence $e$ and $e^{\prime}$ are parallel on $\Gamma_{b}$.

Lemma 12.3 Suppose $\Gamma_{a}$ contains bigons $e_{1} \cup e_{2}$ and $e_{1}^{\prime} \cup e_{2}^{\prime}$, such that $e_{1}, e_{1}^{\prime}$ have label $i$ at $u_{1}$ and $j$ at $u_{2}$, and $e_{2}, e_{2}^{\prime}$ have label $i+1$ at $u_{1}$ and $j+1$ at $u_{2}$. Suppose either

(i) $j \neq i \pm 1$, or

(ii) $\Gamma_{a}$ contains a pair of edges $e_{3}, e_{3}^{\prime}$ with the same label pair $(r, s)$, such that $r, s \notin\{i, i+1, j, j+1\}$ and $C_{3}=e_{3} \cup e_{3}^{\prime}$ form an essential loop on $\hat{F}_{b}$.

Then $C_{1}=e_{1} \cup e_{1}^{\prime}$ and $C_{2}=e_{2} \cup e_{2}^{\prime}$ are inessential on $\hat{F}_{b}$.

Proof. (i) In this case $C_{1} \cap C_{2}=\emptyset$, so they cannot be essential and yet nonhomotopic on $\hat{F}_{b}$, hence by Lemma 2.20 they must be inessential.

(ii) In this case $C_{1}, C_{2}$ lie in the interior of the annulus obtained from $\hat{F}_{b}$ by cutting along $C_{3}$, which again implies that $C_{1}, C_{2}$ cannot be essential and yet non-homotopic on $\hat{F}_{b}$.

An edge is a border edge if it is the first or last edge in a family of parallel edges.

Lemma 12.4 Suppose $s_{k} \neq \pm 1$. Then

(1) $a_{k} \leq n+1$, and

(2) if $a_{k}=n+1$ and $e^{\prime}$ is an edge of $\hat{e}_{j}$ which has the same label pair as that of a border edge $e_{1}$ of $\hat{e}_{k}$, then $e^{\prime}$ is a border edge.

Proof. (1) Assume $a_{k} \geq n+2$. Label the first $n+2$ edges of $\hat{e}_{k}$ successively as $e_{1}, e_{2}, \ldots, e_{n}, e_{n+1}, e_{n+2}$. Let $e_{i}^{\prime}=e_{n+i}$. Since $s_{k} \neq \pm 1, e_{1}, e_{2}, e_{1}^{\prime}, e_{2}^{\prime}$ satisfy Condition (i) in Lemma 12.3, so $e_{1} \cup e_{1}^{\prime}$ is an inessential loop on $\hat{F}_{b}$. By Lemma 
12.2 this implies that $e_{1}$ and $e_{1}^{\prime}$ are parallel on $\Gamma_{b}$, and hence parallel on both graphs, which is a contradiction.

(2) If $e^{\prime}$ is not a border edge then the bigon $e_{1} \cup e_{2}$ and one of the two bigons containing $e^{\prime}$ satisfy the assumption of Lemma 12.3(i), hence $e^{\prime}$ is parallel to $e_{1}$ on $\Gamma_{b}$. Similarly, using the bigon between $e_{n}, e_{n+1}$ and the other bigon containing $e^{\prime}$ one can show that $e^{\prime}$ is also parallel to $e_{n+1}$ on $\Gamma_{b}$, hence $e_{1}, e_{n+1}$ are parallel on both $\Gamma_{a}$ and $\Gamma_{b}$, which is again a contradiction.

Lemma 12.5 Let $\hat{e}, \hat{e}^{\prime}$ be families of at least $n$ parallel edges in $\Gamma_{a}$, and let $i, j, k, l \in \mathbb{Z}_{n}$ be distinct. Then $\Gamma_{a}$ cannot contain both

(i) $i j$-edges $e_{1}, e_{2}, e_{3}$ with $e_{1}, e_{2} \in \hat{e}$ and $e_{3}$ non-equidistant with $e_{1}, e_{2}$; and

(ii) $k l$-edges $e_{1}^{\prime}, e_{2}^{\prime} \in \hat{e}^{\prime}$.

Proof. The edges $e_{1}, e_{2}, e_{3}$ are pairwise non-parallel in $\Gamma_{b}$. Since $e_{1}, e_{2} \in \hat{e}$, no pair of $e_{1}, e_{2}, e_{3}$ cobounds a disk in $\hat{F}_{b}$ by Lemma 12.2. Hence $e_{1}^{\prime}, e_{2}^{\prime}$ cobound a disk in $\hat{F}_{b}$. Since $e_{1}^{\prime} \in E^{\prime}, e_{1}^{\prime}, e_{2}^{\prime}$ are parallel on $\Gamma_{b}$ by Lemma 12.2. This contradicts Lemma 2.2(2).

Two families $\hat{e}, \hat{e}^{\prime}$ of $\Gamma_{a}$ are $A$-conjugate it there are $e \in \hat{e}$ and $e^{\prime} \in \hat{e}^{\prime}$ such that they are anti-parallel on $\Gamma_{b}$ when oriented on $\Gamma_{a}$ from $u_{1}$ to $u_{2}$. They are $P$-conjugate if the $e, e^{\prime}$ above are parallel on $\Gamma_{b}$ as oriented edges. They are conjugate if they are either A-conjugate or P-conjugate.

Lemma 12.6 (1) There exist $\hat{e}_{i}, \hat{e}_{j}$ on $\hat{\Gamma}_{a}$ which are A-conjugate. Moreover, if $a_{i}<(\Delta-3) n$ or $a_{j}<(\Delta-3) n$ then there is another such pair. (The two pairs may have one family in common.)

(2) If $\hat{e}_{i}, \hat{e}_{j}$ are $A$-conjugate then $s_{i} \equiv-s_{j} \bmod n$; moreover, if $a_{i} \geq n$ or $a_{j} \geq n$ then $s_{i} \not \equiv s_{j} \bmod n$.

Proof. (1) Since there are $\Delta n$ edges while $\hat{\Gamma}_{b}$ has at most $3 n$ edges, $\Gamma_{b}$ has at least $(\Delta-3) n$ bigons. The two edges of a bigon in $\Gamma_{b}$ belong to a pair of A-conjugate families $\hat{e}_{i}, \hat{e}_{j}$ on $\Gamma_{a}$. If $a_{i}<(\Delta-3) n$ or $a_{j}<(\Delta-3) n$ then these families cannot contain all the bigons on $\Gamma_{b}$, hence there must be another A-conjugate pair.

(2) If $\hat{e}_{i}, \hat{e}_{j}$ are A-conjugate then by definition there exist $e \in \hat{e}_{i}$ and $e^{\prime} \in \hat{e}_{j}$ which are anti-parallel on $\Gamma_{b}$, hence the label of $e^{\prime}$ at $u_{2}$ is the same as that of $e$ at $u_{1}$, and vice versa. Therefore $s_{i} \equiv-s_{j} \bmod n$.

If we also have $s_{i} \equiv s_{j} \bmod n$ then $s_{i}=0$ or $n / 2$, which is a contradiction to the 2-Cycle Lemma 2.14(3).

Lemma 12.7 Let $\hat{e}=e_{1} \cup \ldots \cup e_{p}$ and $\hat{e}^{\prime}=e_{1}^{\prime} \cup \ldots \cup e_{q}^{\prime}$ be two families of $\Gamma_{a}$, where $p \leq q$.

(1) If $\hat{e}$ and $\hat{e}^{\prime}$ are conjugate then $p \equiv q \bmod 2$.

(2) If $\hat{e}$ and $\hat{e}^{\prime}$ are conjugate and $q \geq p$ then each edge $e_{r}$ is parallel to the edge $e_{r+c}^{\prime}$, where $c=(q-p) / 2$; hence the set of edges in $\hat{e}^{\prime}$ which are parallel to those in $\hat{e}$ lie exactly in the middle of $\hat{e}^{\prime}$. 
(3) If $p+q \equiv 0 \bmod 2 n$ and $\hat{e}, \hat{e}^{\prime}$ are adjacent on $\hat{\Gamma}_{a}$ then they are not A-conjugate.

(4) If $p+q \equiv 0 \bmod 2 n, \hat{e}, \hat{e}^{\prime}$ are adjacent on $\hat{\Gamma}_{a}$, and $J \neq \pm 1$ then they are not conjugate.

Proof. (1) By definition there are edges $e_{i}, e_{j}^{\prime}$ which are parallel in $\Gamma_{b}$.

First consider the case that $\hat{e}, \hat{e}^{\prime}$ are adjacent. Denote by $e(k)$ the endpoints of $e$ at $u_{k}$. We may assume that the first edge $e_{1}^{\prime}$ of $\hat{e}^{\prime}$ is adjacent to the last edge $e_{p}$ of $\hat{e}$ on $\partial u_{1}$. Then the distance from $e_{i}(1)$ to $e_{j}^{\prime}(1)$ is

$$
d_{u_{1}}\left(e_{i}, e_{j}^{\prime}\right)=j+p-i
$$

On $\partial u_{2} e_{q}^{\prime}(2)$ is adjacent to $e_{1}(2)$, so we have

$$
d_{u_{2}}\left(e_{j}^{\prime}, e_{i}\right)=i+q-j
$$

Since $e_{i}, e_{j}^{\prime}$ are parallel positive edges on $\Gamma_{b}$, they are equidistant, hence by Lemma 2.17 we have $d_{u_{1}}\left(e_{i}, e_{j}^{\prime}\right)=d_{u_{2}}\left(e_{j}^{\prime}, e_{i}\right)$, which gives

$$
2(j-i)=q-p
$$

and

$$
2 d=p+q
$$

where $d=d_{u_{1}}\left(e_{i}, e_{j}^{\prime}\right)=d_{u_{2}}\left(e_{j}^{\prime}, e_{i}\right)$. Equation (C) gives $q-p \equiv 0 \bmod 2$.

Now suppose $\hat{e}, \hat{e}^{\prime}$ are not adjacent. Let $\hat{e}^{\prime \prime}$ be the family whose endpoints on $\partial u_{1}$ are between $e_{p}(1)$ and $e_{1}^{\prime}(1)$. Then on $\partial u_{2}$ the endpoints of $\hat{e}^{\prime \prime}$ are also exactly the ones between $e_{q}^{\prime}(2)$ and $e_{1}(2)$. Thus if $\hat{e}^{\prime \prime}$ has $k$ edges then the equations (5) and (6) above become $d=j+k+p-i$ and $d=i+k+q-j$. Therefore again we have $2(j-i)=q-p$, and the result follows.

(2) From equation (7) we have $j=i+(q-p) / 2$. If $i>1$ then the above and the condition $q \geq p$ imply that $j>1$. Since $e_{i}$ is parallel to $e_{j}^{\prime}$ on $\Gamma_{b}$, by Lemma 2.20 applied to the bigons $e_{i-1} \cup e_{i}$ and $e_{j-1}^{\prime} \cup e_{j}^{\prime}$, the loop $e_{i-1} \cup e_{j-1}^{\prime}$ is null-homotopic on $\hat{F}_{b}$, hence by Lemma $12.2 e_{i-1}$ is parallel to $e_{j-1}^{\prime}$ on $\Gamma_{b}$. Similarly, if $i<p$ then the edge $e_{i+1}$ is parallel to $e_{j+1}^{\prime}$ on $\Gamma_{b}$. By induction it follows that every edge $e_{k}$ in $\hat{e}$ is parallel to the edge $e_{k+(q-p) / 2}^{\prime}$.

(3) Assume without loss of generality that $\hat{e}=\hat{e}_{1}$ and $\hat{e}^{\prime}=\hat{e}_{2}$. If they are A-conjugate then the label of $e_{j}^{\prime}$ at $u_{1}$ is the same as the label of $e_{i}$ at $u_{2}$, so $d=d_{u_{1}}\left(e_{i}, e_{j}^{\prime}\right) \equiv s_{1} \bmod n$. Hence equation (8) and the assumption $p+q \equiv 0$ $\bmod 2 n$ gives $s_{1} \equiv d \equiv 0 \bmod n$. Since $n \geq 3$, this is a contradiction to Lemma $2.14(2)$.

(4) By (3) $\hat{e}, \hat{e}^{\prime}$ are not A-conjugate. Assume they are P-conjugate.

If $p+q=4 n$ then by Lemma 2.22(3) we have $p=q=2 n$, and by (2) each $e_{i}$ of $\hat{e}_{1}$ is parallel to the corresponding edge $e_{i}^{\prime}$ of $\hat{e}_{2}$ on $\Gamma_{b}$ for $i=1, \ldots, 2 n$. Since $\hat{e}, \hat{e}^{\prime}$ are not A-conjugate, $e_{i}, e_{i}^{\prime}$ are parallel as oriented edges, with orientation from $u_{1}$ to $u_{2}$. Hence there is another edge $e_{i}^{\prime \prime}$ between them, which cannot 
belong to $\hat{e} \cup \hat{e}^{\prime}$ as otherwise there would be two edges parallel on both graphs, contradicting Lemma 2.2(2). This gives at least $6 n$ edges on $\Gamma_{a}$, which is a contradiction.

Now assume $p+q=2 n$. Let $e, e^{\prime}$ be the edges of $\hat{e}, \hat{e}^{\prime}$ which are parallel on $\Gamma_{b}$ as oriented edges, so they have the same label $k$ at $u_{1}$ for some $k$. The condition $p+q=2 n$ implies that $e, e^{\prime}$ are adjacent among edges labeled $k$ at $u_{1}$. Since $J \neq \pm 1$, they are non-adjacent on $\Gamma_{b}$ among edges labeled 1 at $v_{k}$, hence they belong to a family of at least 5 parallel edges, which is a contradiction to Lemma 2.2(2) because $\hat{\Gamma}_{a}$ has at most 4 edges.

Lemma 12.8 If the jumping number $J= \pm 1$ (in particular if $\Delta=4$ ), then $\Gamma_{a}$ has at most $n+1$ parallel edges.

Proof. Suppose for contradiction that $\hat{e}_{1}$, say, contains edges $e_{1}, \ldots, e_{n+2}$. By Lemma 12.4(1) we may assume that $s_{1}=1$, so the label sequences of these edges are $(1,2, \ldots, n, 1,2)$ at $u_{1}$, and $(2,3, \ldots, n, 1,2,3)$ at $u_{2}$. By Lemma 2.22(1) we may assume that the subgraph of $\Gamma_{b}$ consisting of these edges is as shown in Figure 2.3. Up to symmetry we may assume that the orientation of $\partial v_{i}$ is counterclockwise on Figure 2.3.

Orient edges from $u_{1}$ to $u_{2}$. Denote by $h_{i}, t_{i}$ the head and tail of $e_{i}$, respectively. For $i>1, h_{i-1}$ and $t_{i}$ both have label $i$ on $\Gamma_{a}$, so they are on $\partial v_{i}$. Define $d_{i}=d_{v_{i}}\left(t_{i}, h_{i-1}\right)$, where $i=2, \ldots, n+2$. Note that $d_{i}=1$ implies that the corner from $t_{i}$ to $h_{i-1}$ on $\partial v_{i}$ contains no edge endpoint.

CLAIM 1. $d_{i}=d_{j}$ for $2 \leq i, j \leq n+2$.

Proof. Isotoping on $T_{0}$ along the positive direction of $\partial u_{i}$ moves $h_{1}$ to $h_{2}$ and $t_{2}$ to $t_{3}$, so the distance on $\partial v_{2}$ from $h_{1}$ to $t_{2}$ should be the same as that on $\partial v_{3}$ from $h_{2}$ to $t_{3}$, i.e., $d_{2}=d_{3}$. (Alternatively one may apply Lemma 2.16 to obtain the result.) Similarly we have $d_{i}=d_{i+1}$ for $2 \leq i \leq n+1$.

CLAIM 2. $d_{i}=1$ for $2 \leq i \leq n+2$.

Proof. By assumption we have $J= \pm 1$, so either $d_{v_{2}}\left(h_{1}, h_{n+1}\right)=2$ (when $J=1$ ), or $d_{v_{1}}\left(t_{n+1}, t_{1}\right)=2$ (when $J=-1$ ). In the first case, from Figure 2.3 we see that the tail of $e_{n+2}$ is the only edge endpoint at the corner from $h_{1}$ to $h_{n+1}$, hence $d_{n+2}=1$. Similarly in the second case the head of $e_{n}$ is the only edge endpoint on $\partial v_{1}$ from $t_{n+1}$ to $t_{1}$, hence $d_{n+1}=d_{v_{1}}\left(t_{n+1}, h_{n}\right)=1$. In either case by Claim 1 we have $d_{i}=1$ for all $i$ between 2 and $n+2$.

Let $D$ be the disk face indicated in Figure 2.3. Claim 2 shows that all corners of $D$ except $c_{1}, c_{2}, c_{3}$ shown in the figure contain no edge endpoints.

When $J=1$, we have $d_{v_{1}}\left(t_{1}, t_{n+1}\right)=2$, so there is one edge endpoint in $c_{1}$. Similarly there is one edge endpoint in $c_{3}$. Since $d_{v_{2}}\left(t_{n+2}, t_{2}\right)=2 \Delta-2 \geq 6$, there are at least 4 edge endpoints in $c_{2}$. Thus there would be some trivial loops based at $v_{2}$, contradicting the assumption that $\Gamma_{b}$ has no trivial loops.

When $J=-1, d_{v_{1}}\left(t_{1}, t_{n+1}\right)=d_{v_{3}}\left(h_{2}, h_{n+2}\right)=2 \Delta-2 \geq 6$, and $d_{v_{2}}\left(t_{n+2}, t_{2}\right)=$ 2 , so there are at least 5 edge endpoints in each of $c_{1}$ and $c_{3}$, and no edge endpoints in $c_{2}$. It follows that $D$ contains at least 5 interior edges, all parallel to 
each other, two of which would then be parallel on both graphs, contradicting Lemma 2.2(2).

Lemma 12.9 $\Gamma_{a}$ has at most $n+2$ parallel edges.

Proof. Assume to the contrary that $\hat{e}_{1} \supset e_{1} \cup \ldots \cup e_{n+3}$. By Lemma 12.4(1) we may assume without loss of generality that $s_{1}=1$. By Lemma 2.22(1) the first $n+2$ edges appear in $\Gamma_{b}$ as shown in Figure 2.3

First assume $n \geq 4$. Orient edges of $\Gamma_{a}$ from $u_{1}$ to $u_{2}$, and denote by $e(h), e(t)$ the head and tail of an edge $e$, respectively. From Figure 2.3 we see that $\left(e_{2}(h), e_{n+2}(h), e_{3}(t)\right)$ is a positive triple, hence by Lemma $2.21(2)$ the triple $\left(e_{3}(h), e_{n+3}(h), e_{4}(t)\right)$ is also positive, so the head of $e_{n+3}$ lies on the inner circle in Figure 2.3. Note that $e_{n+2}$ shields this edge endpoint from the outside circle of the annulus in Figure 2.3, hence the tail of $e_{n+3}$ also lies in the inner circle in the figure, therefore $e_{n+3}$ is parallel to $e_{3}$ on $\Gamma_{b}$, which is a contradiction as they cannot be parallel on both graphs.

Now consider the case $n=3$. By Lemma 2.22(3) we may assume $a_{i} \leq 6$, and $a_{1}=n+3=6$. By Lemma 12.8 we may assume that $\Delta=5$ and the jumping number $J \neq \pm 1$. Also, $a_{j} \neq 5$, otherwise by Lemma 12.7(1) the 11 edges in $\hat{e}_{1} \cup \hat{e}_{j}$ would be mutually non-parallel on $\Gamma_{b}$, contradicting the fact that $\hat{\Gamma}_{b}$ has at most $3 n=9$ edges (Lemma 2.5). One can now check that the following are the only possible values of $\left(a_{1}, a_{2}, a_{3}, a_{4}\right)$ up to symmetry, where * indicates any possible value. Let $s=s_{1}$. Then the other $s_{i}$ can be calculated using Lemma 12.1. The second quadruple in the following list indicates the values of $\left(s_{1}, s_{2}, s_{3}, s_{4}\right)$.

$\begin{array}{lll}(1) & (6,6, *, *) & (s, s, *, *) \\ (2) & (6,1,6,2) & (s, s-1, s+1, s-1) \\ (3) & (6,1,4,4) & (s, s-1, s, s+1) \\ (4) & (6,4,3,2) & (s, s-1, s+1, s-1) \\ (5) & (6,4,1,4) & (s, s-1, s, s+1) \\ (6) & (6,3,2,4) & (s, s, s+1, s+1) \\ (7) & (6,3,4,2) & (s, s, s-1, s-1) \\ (8) & (6,3,3,3) & (s, s, s, s)\end{array}$

In case (1) by Lemma 12.7(4) the 12 edges in $\hat{e}_{1} \cup \hat{e}_{2}$ are mutually non-parallel on $\Gamma_{b}$, which is impossible because $\hat{\Gamma}_{b}$ contains at most $3 n=9$ edges. Case (8) is impossible by Lemma 12.6. Also, Lemma 2.14(3) implies that $s_{i} \not \equiv 0 \bmod 3$ if $a_{i} \geq 2$, which can be applied to exclude cases (2), (4) and (5).

In case (6) and (7), by Lemma 12.7(1) $\hat{e}_{2}$ is not conjugate to $\hat{e}_{1}, \hat{e}_{3}$ or $\hat{e}_{4}$, so $\hat{e}_{1} \cup \hat{e}_{2}$ represents all 9 edges in $\hat{\Gamma}_{b}$, hence each of $\hat{e}_{3}$ and $\hat{e}_{4}$ must be conjugate to $\hat{e}_{1}$. By Lemma 12.7(2) the two middle edges of $\hat{e}_{1}$ are parallel to the middle edges in each of $\hat{e}_{3}$ and $\hat{e}_{4}$, so $\hat{e}_{3}, \hat{e}_{4}$ are conjugate. Since $a_{3}+a_{4}=6=2 n$ and $J \neq \pm 1$, this is a contradiction to Lemma 12.7(4).

In case (3), by Lemma 2.14(3) we have $s=1$. Since $a_{1}+a_{3}=a_{1}+a_{4}=10$ while $\hat{\Gamma}_{b}$ has at most 9 edges, each of $\hat{e}_{3}, \hat{e}_{4}$ must have an edge parallel to some 
edge of $\hat{e}_{1}$ on $\Gamma_{b}$. By Lemma 12.7(2) this implies that each edge of $\hat{e}_{3} \cup \hat{e}_{4}$ is parallel to one of the 4 middle edges in $\hat{e}_{1}$. Note that the edge $e^{\prime}$ in $\hat{e}_{2}$ is a loop based at $v_{1}$ in $\Gamma_{b}$, which cannot be parallel to any other edge on $\Gamma_{b}$. Therefore $\Gamma_{b}$ has exactly 7 families. Moreover, if we let $e_{1}, e_{6}$ be the first and last edges in $\hat{e}_{1}$ then each of $e_{1}, e_{6}, e^{\prime}$ forms a single family.

Now consider the graph in Figure 2.4. Clearly there is only one possible position for $e^{\prime}$, which has exactly one endpoint on the corner from the tail of $e_{1}$ to the head of $e_{6}$. By the above there are no other edge endpoints in this corner, which is a contradiction because the label of the tail of $e_{1}$ is 1 while the label of the head of $e_{6}$ is 2 , so the number of edge endpoints between them must be even.

Lemma 12.10 Suppose $n \geq 4$. Then $\Delta=4$ and $\Gamma_{a}$ has at most $n+1$ parallel edges.

Proof. We need only show that $\Delta=4$. The second statement will then follow from Lemma 12.8 .

Suppose to the contrary that $\Delta=5$. First assume that $a_{i}<n+2$ for all $i$. Then $\Delta n=5 n \leq 4(n+1)$, so $n=4$, and $\Gamma_{a}=(5,5,5,5)$. By Lemma 2.3(1) $s_{1}$ is coprime with $n=4$, so we may assume without loss of generality that $s_{1}=1$. Thus the label sequences of $\hat{e}_{1}$ are $(1,2,3,4,1)$ at $u_{1}$ and $(2,3,4,1,2)$ at $u_{2}$. One can check that the label sequences of $\hat{e}_{3}$ are $(3,4,1,2,3)$ at $u_{1}$, and $(4,1,2,3,4)$ at $u_{2}$. This contradicts Lemma 12.5 with $e_{1}, e_{2}$ the two 12-edges in $\hat{e}_{1}, e_{3}$ the 12-edge in $\hat{e}_{3}$, and $e_{1}^{\prime}, e_{2}^{\prime}$ the two 34-edges in $\hat{e}_{3}$.

We may now assume without loss of generality that $a_{1}>n+1$. By Lemma 12.9 we must have $a_{1}=n+2$. By Lemma 12.4(1) we have $s_{1}= \pm 1$.

CLAIM 1. $a_{2}, a_{4} \leq n+1$.

Proof. Suppose $a_{2}=n+2$. Then $s_{2}= \pm 1$ by Lemma 12.4(1). Also by Lemma 12.1 we have

$$
s_{1}-s_{2} \equiv a_{1}+a_{2} \equiv 4 \quad \bmod n
$$

Hence either $n=4$ and $s_{1}=s_{2}$ (=1 say), or $n=6, s_{1}=-1$ and $s_{2}=1$. In either case one can check that there is a pair of parallel 12-edges $e_{1}, e_{2}$ in $\hat{e}_{1}$, a 12-edge $e_{3}$ in $\hat{e}_{2}$ which is not equidistant to $e_{1}, e_{2}$, and a pair of parallel 34-edges $e_{1}^{\prime}, e_{2}^{\prime}$ in $\hat{e}_{2}$. This is a contradiction to Lemma 12.5.

Hence $a_{2} \leq n+1$. A symmetric argument shows that $a_{4} \leq n+1$.

We now have $5 n \leq 2(n+2)+2(n+1)$, giving $n \leq 6$. Also if $n=6$ then $\Gamma_{a}=(8,7,8,7)$.

CLAIM 2. $n=5$.

Proof. Otherwise we have $n=4$ or 6 . If $a_{2}=n+1$ then Lemma 12.1 gives $s_{2}=s_{1}-a_{1}-a_{2}= \pm 1-(n+1)-(n+2) \equiv 0 \bmod 2$, which is a contradiction to the fact that the transition function of a family of more than $n$ edges must be transitive (Lemma 2.3(1)). Therefore we have $a_{2} \leq n$. Similarly for $a_{4}$. This rules out the case $n=6$. 
When $n=4$ we must have $\Gamma_{a}=(6,4,6,4)$. Assume without loss of generality that $s_{1}=1$. We now apply Lemma 12.5 with $e_{1}, e_{2}$ the 12 -edges in $\hat{e}_{1}, e_{3}$ the 12-edge in $\hat{e}_{3}$, and $e_{1}^{\prime}, e_{2}^{\prime}$ the 34-edges in $\hat{e}_{3}$.

CLAIM 3. If $n=5$ then $a_{3} \neq 7$.

Proof. Otherwise by Claim 1 we have $\left(a_{2}, a_{4}\right)=(6,5)$ or $(5,6)$, so $a_{4}-a_{2}= \pm 1$. We may assume that $s_{1}=1$. By Lemma 12.1 we have

$$
s_{3} \equiv s_{1}-a_{2}+a_{4}=1 \mp 1=0 \text { or } 2 \bmod 5
$$

which contradicts the fact that $s_{3}= \pm 1 \bmod n($ Lemma 12.4(1)).

The only possibility left is that $n=5$ and $\Gamma_{a}=(7,6,6,6)$. We may assume $s_{1}=1$. Then this can be ruled out by applying Lemma 12.5 with $e_{1}, e_{2}$ the 12-edges in $\hat{e}_{1}, e_{3}$ the 12-edge in $\hat{e}_{3}$, and $e_{1}^{\prime}, e_{2}^{\prime}$ the 45 -edges in $\hat{e}_{3}$.

Lemma $\mathbf{1 2 . 1 1}$ (a) $\Gamma_{a}$ has at most $n+1$ parallel edges.

(b) $\Delta=4$.

Proof. (a) This follows from Lemmas 12.8 and 12.10 if either $J= \pm 1$, or $\Delta=4$, or $n \geq 4$. Hence we may assume that $\Delta=5, J \neq \pm 1$, and $n=3$. By Lemma 12.9 we have $a_{i} \leq n+2=5$. Thus the possible values of $\left(a_{1}, a_{2}, a_{3}, a_{4}\right)$ are given below. The second quadruple gives $\left(s_{1}, s_{2}, s_{3}, s_{4}\right)$, calculated as functions of $s=s_{1}$, using Lemma 12.1 .

$\begin{array}{lll}(1) & (5,5,5,0) & (s, s-1, s+1,-) \\ (2) & (5,5,4,1) & (s, s-1, s-1, s) \\ (3) & (5,3,2,5) & (s, s+1, s-1, s+1) \\ (4) & (5,4,5,1) & (s, s, s, s) \\ (5) & (5,4,4,2) & (s, s, s+1, s+1) \\ (6) & (5,4,3,3) & (s, s, s-1, s-1) \\ (7) & (5,4,2,4) & (s, s, s, s) \\ (8) & (5,3,5,2) & (s, s+1, s-1, s+1) \\ (9) & (5,3,4,3) & (s, s+1, s, s-1)\end{array}$

Cases (1), (3), (8) and (9) are impossible because there is an $i$ such that $a_{i} \geq 2$ and $s_{i}=0$, contradicting Lemma 2.14(3). Cases (4) and (7) contradict Lemma 12.6 .

In case (2), by Lemma 12.7(1) the edges in $\hat{e}_{3}$ are not parallel to those in $\hat{e}_{1} \cup \hat{e}_{4}$ on $\Gamma_{b}$, and by Lemma 12.7(4) the edge in $\hat{e}_{4}$ is not parallel to those in $\hat{e}_{1}$. Thus the 10 edges in $\hat{e}_{1} \cup \hat{e}_{3} \cup \hat{e}_{4}$ are mutually non-parallel on $\Gamma_{b}$, contradicting the fact that $\hat{\Gamma}_{b}$ has at most $3 n$ edges (Lemma 2.5). Similarly, in case (5) the edges in $\hat{e}_{1} \cup \hat{e}_{3} \cup \hat{e}_{4}$ are mutually non-parallel on $\Gamma_{b}$, and in case (6) the edges in $\hat{e}_{2} \cup \hat{e}_{3} \cup \hat{e}_{4}$ are mutually non-parallel on $\Gamma_{b}$, which lead to the same contradiction.

(b) Assume $\Delta=5$. By Lemma 12.10 we have $n=3$, and by (a) we have $a_{i} \leq 4$, hence the weights of $\hat{e}_{i}$ must be $(4,4,4,3)$ up to symmetry, and the transition numbers are $(s, s+1, s-1, s+1)$. This is a contradiction to Lemma 
2.14(2) because one of the families has $s_{i}=0$ and hence is a set of co-loops.

Lemma 12.12 Suppose $\Delta=4$. Let $e, e^{\prime}$ be edges of $\Gamma_{a}$ with label $i$ at vertex $u_{1}$ and $j$ at $u_{2}, i \neq j$, where the $i$-labels of $e, e^{\prime}$ at $u_{1}$ are not adjacent among all $i$-labels at $u_{1}$. Suppose also that e belongs to a family of at least $n$ parallel edges of $\Gamma_{a}$. Then $e \cup e^{\prime}$ forms an essential loop on the torus $\hat{F}_{b}$.

Proof. Note that in this case the jumping number $J= \pm 1$, so the assumption that the $i$-labels of $e, e^{\prime}$ at $u_{1}$ are not adjacent implies that the 1-labels of $e_{1}, e_{1}^{\prime}$ at the vertex $v_{i}$ in $\Gamma_{b}$ are not adjacent among all 1-labels. By assumption $e$ belongs to a family of at least $n$ parallel edges of $\Gamma_{a}$, so if $e \cup e^{\prime}$ is inessential on $\hat{F}_{b}$ then by Lemma $12.2 e_{1}$ and $e_{1}^{\prime}$ are parallel on $\Gamma_{b}$, which gives rise to at least 5 parallel edges in $\Gamma_{b}$, contradicting Lemma 2.2(2) because $\hat{\Gamma}_{a}$ has at most 4 edges.

Up to symmetry we may assume that $a_{1} \geq a_{3}, a_{2} \geq a_{4}$, and $a_{1}+a_{3} \geq a_{2}+a_{4}$. Since $a_{i} \leq n+1$, the possibilities for $\Gamma_{a}$ are listed below. The second quadruple indicates the values of $s_{i}$, calculated in terms of $s=s_{1}$ using Lemma 12.1.

(7) $\quad(n+1, n-1, n+1, n-1)$

(8) $\quad(n, n, n, n)$

$$
\begin{aligned}
& (s, s-2, s-4, s-2) \\
& (s, s-2, s-3, s-1) \\
& (s, s-2, s-2, s) \\
& (s, s-1, s-2, s-1) \\
& (s, s-1, s-1, s) \\
& (s, s-1, s, s+1) \\
& (s, s, s, s) \\
& (s, s, s, s)
\end{aligned}
$$

Lemma 12.13 Cases (4), (5), (6), (7), (8) are impossible.

Proof. In case (4) $\hat{e}_{1}, \hat{e}_{3}$ are not A-conjugate to $\hat{e}_{2}, \hat{e}_{4}$ by Lemma 12.7(1). Since $a_{4}<n$, by Lemma 12.6(1) $\hat{e}_{2}, \hat{e}_{4}$ cannot be the only A-conjugate pair, hence $\hat{e}_{1}$ must be A-conjugate to $\hat{e}_{3}$. Since they have the same number of edges, by Lemma 17.2 the first edge $e$ of $\hat{e}_{1}$ is parallel to the first edge $e^{\prime}$ of $\hat{e}_{3}$. Since $e, e^{\prime}$ have labels 1,2 at $u_{1}$, respectively, the label of $e$ at $u_{2}$ is 2 , hence $s=1$. Now $\hat{e}_{2}$ is a family of at least 3 co-loops, contradicting Lemma 2.14(2).

In case (5), by Lemma 12.7(1) $\hat{e}_{1}$ can only be conjugate to $\hat{e}_{4}$ and $\hat{e}_{2}$ to $\hat{e}_{3}$, but since $a_{4}<n$, by Lemma 12.6(1) $\hat{e}_{2}$ must be A-conjugate to $\hat{e}_{3}$. Since $a_{2}+a_{3}=2 n$, this is a contradiction to Lemma 12.7(3).

For the same reason, in case (6) $\hat{e}_{2}$ must be A-conjugate to $\hat{e}_{4}$. By Lemma 12.7(2) the first edge $e_{1}$ of $\hat{e}_{2}$ must be parallel to the first edge $e_{1}^{\prime}$ of $\hat{e}_{4}$. Examining the labeling we see that they have labels 2 and 1 at $u_{1}$, respectively, so the label of $e_{1}$ at $u_{2}$ is 1 , hence $s_{2}=-1$. It follows that $s_{1}=s=0$, which is a contradiction to Lemma 2.3(1).

Cases (7) and (8) are impossible by Lemma 12.6 . 
Lemma 12.14 Case (1) is impossible.

Proof. Since $a_{4}<n$, by Lemma 12.6(1) two of the first three families are $A$-conjugate. Up to symmetry we may assume that $\hat{e}_{1}$ is $A$-conjugate to $\hat{e}_{2}$ or $\hat{e}_{3}$. By Lemma 12.7(2) the first edges of the above conjugate pair must have the same label pair. Examining the labels of these edges on $u_{1}$ we see that $s=1$ if $\hat{e}_{1}$ is $A$-conjugate to $\hat{e}_{2}$, and $s=2$ if $\hat{e}_{1}$ is $A$-conjugate to $\hat{e}_{3}$. The second case cannot happen because then $\hat{e}_{2}$ would be a set of at least 3 co-loops, contradicting Lemma 2.14(2).

The graph $\Gamma_{a}$ is now shown in Figure 12.1. If $n \geq 5$ then there are bigons $e_{1} \cup e_{2}$ in $\hat{e}_{2}$ and $e_{1}^{\prime}, e_{2}^{\prime}$ in $\hat{e}_{4}$ with labels 4,5 at $u_{1}$ and 5,6 at $u_{2}(6=1$ when $n=5)$. Note also that there is a pair of parallel 23-edges $e_{3} \cup e_{3}^{\prime}$ in $\hat{e}_{2}$. By Lemma 12.3(ii), these conditions imply that $e_{1} \cup e_{1}^{\prime}$ is inessential on $\hat{F}_{b}$, which contradicts Lemma 12.12 .

When $n=4$, there is a pair of 14-edges $e_{1}, e_{2}$ in $\hat{e}_{1}$, a 14-edge $e_{3}$ in $\hat{e}_{4}$, and a pair of 23 -edges in $\hat{e}_{2}$. Note that $e_{3}$ is not equidistant to $e_{1}, e_{2}$. This leads to a contradiction to Lemma 12.5 .

When $n=3, s_{i}=0$ for some $i=1,2,3$, so one of the first three families contains 4 co-loop edges, which is a contradiction to the 3-Cycle Lemma.

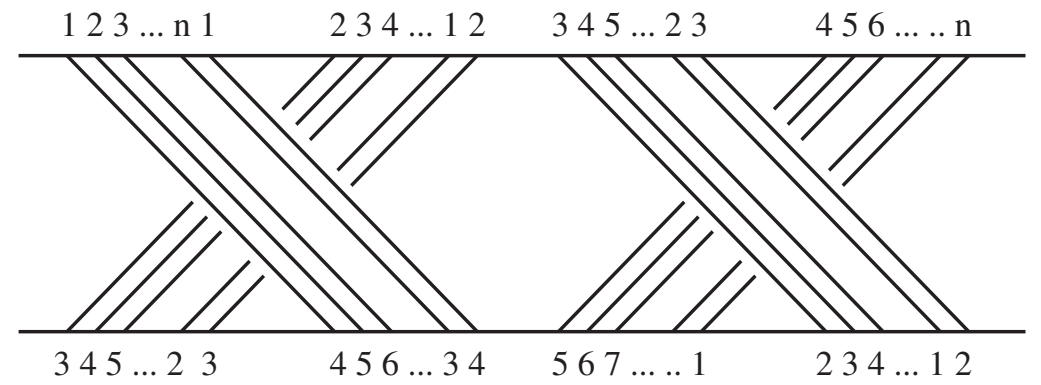

Figure 12.1

Lemma 12.15 Case (2) is impossible.

Proof. In this case $a_{1} \equiv a_{2} \not \equiv a_{3} \equiv a_{4} \bmod 2$, so by Lemma 12.7(1) no edge in $\hat{e}_{1} \cup \hat{e}_{2}$ is parallel to an edge in $\hat{e}_{3} \cup \hat{e}_{4}$. Since $\Gamma_{b}$ contains at least $n$ bigons while $\hat{e}_{3} \cup \hat{e}_{4}$ contributes at most $a_{4}=n-2$ bigons on $\Gamma_{b}$, it follows that some edge in $\hat{e}_{2}$ is parallel to an edge in $\hat{e}_{1}$ on $\Gamma_{b}$. Since $a_{1}=a_{2}$, by Lemma 12.7(2) this implies that the first edge $e_{1}$ of $\hat{e}_{1}$ is parallel to the first edge $e_{1}^{\prime}$ of $\hat{e}_{2}$ on $\Gamma_{b}$. In particular, they must have the same label pair. Since $e_{1}$ has label 1 at $u_{1}$ and $e_{1}^{\prime}$ has label 2 at $u_{1}$, we see that $e_{1}$ has label 2 at $u_{2}$, hence $s=1$. Since $s_{4}=s-1=0$, this is a contradiction to Lemma 2.14(3) unless $a_{4}=n-2<2$, i.e. $n \leq 3$. 
Now suppose $n=3$. Let $e_{1}, e_{2}$ be the two 12-edges in $\hat{e}_{1}$. Note that there is a 12-edge $e_{3}$ in $\hat{e}_{3}$, which by the above is not parallel to any edge in $\hat{e}_{1}$, hence $e_{1}, e_{2}, e_{3}$ cut $\hat{F}_{b}$ into a disk. Now $\hat{e}_{4}$ is a loop based at $v_{3}$ in $\Gamma_{b}$, so it must be a trivial loop. This is a contradiction because $\Gamma_{b}$ contains no trivial loop.

Lemma 12.16 Case (3) is impossible.

Proof. We claim that $s=1$. By Lemma 12.6(1) one of $\hat{e}_{1}, \hat{e}_{2}$ is A-conjugate to some other $\hat{e}_{j}$. Because of symmetry we may assume that $\hat{e}_{1}$ is conjugate to some $\hat{e}_{j}$. If $j=2$ then by Lemma 12.7(2) the first edge $e_{1}$ of $\hat{e}_{1}$ is parallel on $\Gamma_{b}$ to the first edge $e_{1}^{\prime}$ of $\hat{e}_{2}$, which has label 2 at $u_{1}$, hence $e_{1}$ has label 2 at $u_{2}$, so $s=s_{1}=1$. Similarly, if $j=3$ then by Lemma 12.7(2), $a_{1}=n+1$ and $a_{3}=n-1$ implies that the second edge $e_{2}$ of $\hat{e}_{1}$ is parallel on $\Gamma_{b}$ to the first edge of $\hat{e}_{3}$, which has label 3 at $u_{1}$, hence $e_{2}$ has label pair (23), which again implies that $s=1$. The case $j=4$ is impossible by Lemma 12.6(2). The graph $\Gamma_{a}$ is now shown in Figure 12.2.

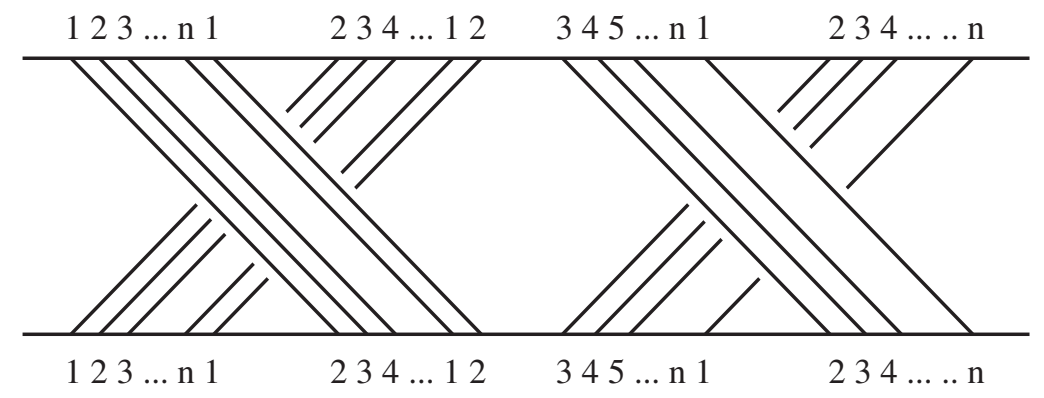

Figure 12.2

There are four edges $e_{1}^{\prime}, \ldots, e_{4}^{\prime}$ with label pair $(2,3)$, where $e_{i}^{\prime} \in \hat{e}_{i}$. One can check on Figure 12.2 that they are all equidistant to each other. We claim that they are all parallel in $\Gamma_{b}$.

The first $n$ edges of $\hat{e}_{1}$ form a loop $C$ on $\hat{F}_{b}$. Let $a_{1}, a_{2}, a_{3}$ be the first three edges of $\hat{e}_{1}$, oriented from $u_{1}$ to $u_{2}$, and let $a_{i}(t), a_{i}(h)$ be the tail and head of $a_{i}$, respectively. Then as in the proof of Lemma 2.22(1), one can show that $d_{v_{2}}\left(a_{1}(h), a_{2}(t)\right)=d_{v_{3}}\left(a_{2}(h), a_{3}(t)\right)$. In other words, the corners at $v_{2}, v_{3}$ on one side of the above loop contain the same number of edge endpoints. Since $e_{2}^{\prime}$ is equidistant to $e_{1}^{\prime}=a_{2}$, we have $d_{v_{2}}\left(a_{2}(t), e_{2}^{\prime}(h)\right)=d_{v_{3}}\left(e_{2}^{\prime}(t), a_{2}(h)\right)$, hence the two endpoints of $e_{2}^{\prime}$ lie on the same side of the loop $C$. It follows that $e_{2}^{\prime}$ is parallel to $e_{1}^{\prime}$. Similarly, $e_{3}^{\prime}, e_{4}^{\prime}$ are also parallel to $e_{1}^{\prime}$. This proves the claim above.

Among the four parallel edges $e_{1}^{\prime}, \ldots, e_{4}^{\prime}$, at least one of $e_{3}^{\prime}, e_{4}^{\prime}$ is adjacent to $e_{1}^{\prime}$ or $e_{2}^{\prime}$ on $\Gamma_{b}$. Because of symmetry we may assume without loss of generality that $e_{3}^{\prime}$ is adjacent to $e_{1}^{\prime}$ or $e_{2}^{\prime}$. Relabel it as $e_{3}$. 
Note that $e_{3}$ is a border edge. There is a face $D$ of $\Gamma_{a}$ with $\partial D=e_{1} \cup e_{2} \cup e_{3} \cup$ $e_{4}$, see Figure 12.3. Let $\alpha$ be the $\operatorname{arc}$ in $D$ connecting the middle points of $e_{2}, e_{4}$. Since $e_{3}=e_{3}^{\prime}$ is parallel and adjacent to $e_{1}^{\prime}$ or $e_{2}^{\prime}$ and $e_{1}^{\prime}, e_{2}^{\prime}$ are non-border edges in $\Gamma_{a}$, the face $D$ has a bigon as a coupling face. (See Section 2 for definition.) It follows from Lemma 2.15 that the surface $F_{a}$ can be isotoped rel $\partial$ so that the new intersection graph $\Gamma_{a}^{\prime}$ is obtained from $\Gamma_{a}$ by deleting $e_{2}, e_{4}$ and replacing them with two edges parallel to $e_{1}, e_{3}$ respectively. The first family of $\Gamma_{a}^{\prime}$ has $n+2$ edges, which is a contradiction to Lemma 12.8 .

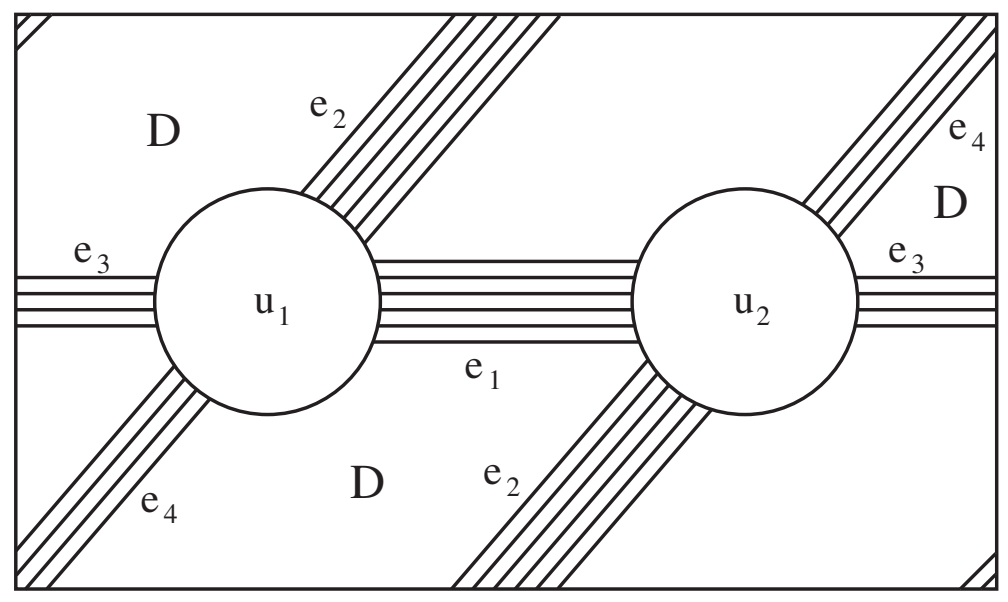

Figure 12.3

Proposition 12.17 The case that $n_{a}=2, n=n_{b} \geq 3$ and $\Gamma_{b}$ positive, is impossible.

Proof. We have shown that $\Gamma_{a}$ has 8 possibilities. These have been ruled out in Lemmas $12.13-12.16$.

\section{The case $n_{a}=2, n_{b}>4, \Gamma_{1}, \Gamma_{2}$ non-positive, and $\max \left(w_{1}+w_{2}, w_{3}+w_{4}\right)=2 n_{b}-2$}

Suppose $n_{a} \leq 2$ and $n=n_{b} \geq 4$. In Section 12 it has been shown that $\Gamma_{b}$ cannot be positive. In sections $13-16$ we will discuss the case that $\Gamma_{b}$ is non-positive. The result will be given in Propositions 14.7 and 16.8 .

As before, we will use $n$ to denote $n_{b}$.

Lemma 13.1 Suppose $n_{a}=2, n \geq 4$, and $\Gamma_{1}, \Gamma_{2}$ are non-positive.

(1) The reduced graph $\hat{\Gamma}_{a}$ is a subgraph of the graph shown in Figure 13.1.

(2) Let $w_{i}$ be the weight of $\hat{e}_{i}$. Then up to relabeling we may assume $w_{3}+$ $w_{4} \leq w_{1}+w_{2}$, and $w_{1}+w_{2}=2 n-2$ or $2 n$. 


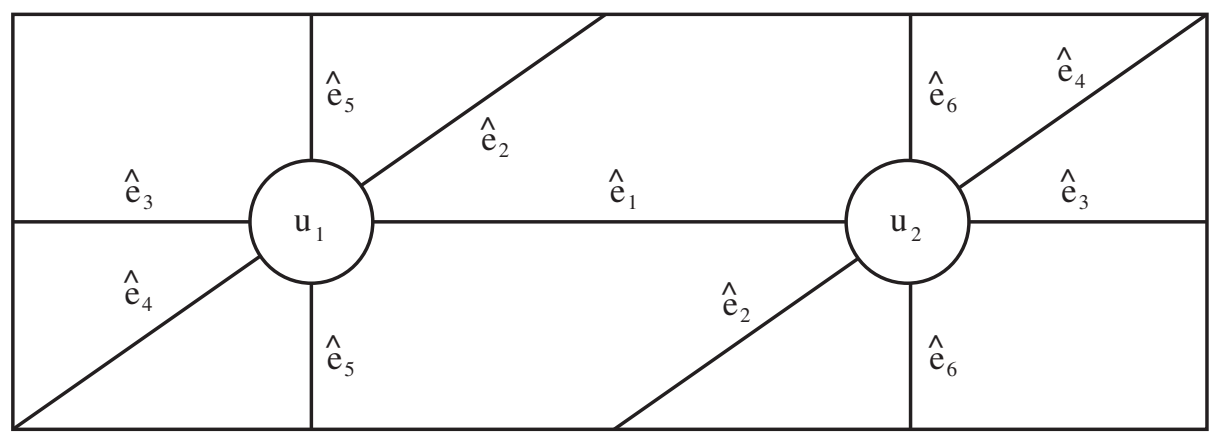

Figure 13.1

Proof. (1) First note that the number of loops in $\Gamma_{a}$ at $u_{1}$ is the same as that at $u_{2}$, because they have the same valence and the same number of non-loop edges. If $\hat{\Gamma}_{a}$ has two loops based at $u_{1}$ then they cut the torus into a disk, so there is no loop at $u_{2}$, which would be a contradiction to the above. Therefore $\hat{\Gamma}_{a}$ has at most one loop edge at each vertex. If there is no loop at $u_{i}$ then $\hat{\Gamma}_{a}$ has at most four edges connecting $u_{1}$ to $u_{2}$. If there is one loop of $\hat{\Gamma}_{a}$ at each $u_{i}$ then these cut the torus into two annuli, each containing at most two edges of $\hat{\Gamma}_{a}$. In either case $\hat{\Gamma}_{a}$ is a subgraph of that in Figure 13.1.

(2) Up to relabeling we may assume that $w_{1}+w_{2} \geq w_{3}+w_{4}$. Since $\Gamma_{b}$ is non-positive, by Lemma 2.3(1) we have $w_{i} \leq n$, hence $w_{1}+w_{2} \leq 2 n$.

Assume $w_{1}+w_{2}=2 n-k$ and $k>0$. Then $w_{5} \geq\left(4 n-\left(w_{1}+\ldots+w_{4}\right)\right) / 2 \geq k$. Note that the set of $k$ edges $e_{1} \cup \ldots \cup e_{k}$ of $\hat{e}_{5}$ adjacent to $\hat{e}_{1} \cup \hat{e}_{2}$ has the same set of labels at each of its two ends. Hence by Lemma $2.4 \hat{e}_{5}$ contains a Scharlemann bigon, so by Lemma 2.2(4) and the parity rule $k$ must be even. If $k \geq 4$ then $e_{1} \cup \ldots \cup e_{k}$ contains an extended Scharlemann bigon, which is a contradiction to Lemma 2.2(6). Hence $k=2$.

If $\Gamma_{b}$ has a Scharlemann cycle then by Lemma 2.2(4) the surface $\hat{F}_{a}$ is separating, cutting $M\left(r_{a}\right)$ into a black region and a white region. Two Scharlemann cycles of $\Gamma_{b}$ have the same color if the disks they bound lie in the same region.

Lemma 13.2 Suppose $n_{a}=2$ and $n \geq 1$.

(1) If $e_{1} \cup e_{2}$ and $e_{1}^{\prime} \cup e_{2}^{\prime}$ are two Scharlemann bigons of $\Gamma_{b}$ of the same color, then either (i) up to relabeling $e_{i}$ is parallel to $e_{i}^{\prime}$ on $\Gamma_{a}$ for $i=1,2$, or (ii) $\Gamma_{a}$ is kleinian, and the four edges $e_{1}, e_{2}, e_{1}^{\prime}, e_{2}^{\prime}$ are mutually non-parallel on $\Gamma_{a}$.

(2) If $\Gamma_{b}$ has four parallel positive edges then $\Gamma_{a}$ is kleinian.

Proof. (1) If the four edges are in two families of $\Gamma_{a}$ then (i) holds. If they are in three families, i.e., $e_{1}$ is parallel to $e_{1}^{\prime}$ but $e_{2}$ is not parallel to $e_{2}^{\prime}$, then the nontrivial loop $e_{2} \cup e_{2}^{\prime}$ on $\hat{F}_{a}$ is homotopic in $M\left(r_{a}\right)$ to the trivial loop $e_{1} \cup e_{1}^{\prime}$, which contradicts the incompressibility of $\hat{F}_{a}$.

Now assume that they are mutually non-parallel. Let $G$ be the subgraph of $\Gamma_{a}$ consisting of these four edges and the two vertices of $\Gamma_{a}$. Let $B$ be the 
side of $\hat{F}_{a}$ which contains the two Scharlemann bigons. Shrinking the Dehn filling solid torus $V_{a}$ to its core $K_{a}$ and cutting $B$ along $\hat{F}_{a}$ and then along the two Scharlemann bigons, we obtain a manifold whose boundary consists of the two disk faces of $G$ and two copies of the two Scharlemann bigons, which is a sphere, so by the irreducibility of $M\left(r_{a}\right)$ it bounds a 3 -ball. It follows that $B$ is a twisted $I$-bundle over a Klein bottle $K$, and $K$ intersects $K_{a}$ at a single point. Therefore by Lemma $2.12 \Gamma_{a}$ is kleinian.

(2) If $\Gamma_{b}$ has four parallel positive edges then they form two Scharlemann bigons of the same color. By Lemma 2.2(2) no two of these edges are parallel on $\Gamma_{a}$, hence by (1) $\Gamma_{a}$ is kleinian.

In the remainder of this section we assume that $\Gamma_{a}, \Gamma_{b}$ are non-positive, $n_{a}=2, n=n_{b}>4$, and $\max \left(w_{1}+w_{2}, w_{3}+w_{4}\right)=2 n-2$. We may assume without loss of generality that $w_{3}+w_{4} \leq w_{1}+w_{2}=2 n-2$. Since $w_{1}+\ldots+$ $w_{4}+2 w_{5}=\Delta n \geq 4 n$, we have $w_{5}=w_{6} \geq 2$. Let $\alpha_{1} \cup \alpha_{2}$ (resp. $\beta_{1} \cup \beta_{2}$ ) be the two edges of $\hat{e}_{5}$ (resp. $\hat{e}_{6}$ ) adjacent to $\hat{e}_{1} \cup \hat{e}_{2}$. Note that these are Scharlemann bigons, hence $\hat{F}_{b}$ is separating, and $n$ is even. Without loss of generality we may assume that $\alpha_{1} \cup \alpha_{2}$ is a (12)-Scharlemann bigon. Assume that $\beta_{1} \cup \beta_{2}$ is a $(k, k+1)$-Scharlemann bigon.

Lemma 13.3 (1) $k$ is even if and only if $w_{2}=n-1$.

(2) $\{1,2\} \cap\{k, k+1\}=\emptyset$.

Proof. (1) This follows from the parity rule. Orient $u_{1}$ counterclockwise and $u_{2}$ clockwise in Figure 13.1. If $w_{2}=n-1$ then the first edge of $\hat{e}_{2}$ has label 2 at $u_{1}$ and $k+2$ at $u_{2}$, so by the parity rule $k$ must be even. Similarly if $w_{2}=n$ or $n-2$ then $k$ is odd.

(2) If $k=1$ then by (1) we have $w_{2}=n$ or $n-2$. In the first case the edges of $\hat{e}_{1}$ would be co-loops, while in the second case the edges of $\hat{e}_{2}$ would be co-loops. If $k=2$ then $w_{1}=w_{2}=n-1$ and the edges of $\hat{e}_{1}$ are co-loops. Similarly if $k=n$ then the edges of $\hat{e}_{2}$ are co-loops. Since $n-2>2$, all cases contradict Lemma 2.14(2) because the above would imply that there are at least three parallel co-loop edges.

Lemma 13.4 Suppose $w_{1}=w_{2}=n-1$. Then for $i=1,2$, the edges of $\hat{e}_{i}$ on $\Gamma_{b}$ form a cycle $C_{i}$ and a chain $C_{i}^{\prime}$ disjoint from $C_{i}$. Moreover, the vertices of $C_{1}\left(C_{2}^{\prime}\right)$ are the set of $v_{j}$ with $j$ odd, while the vertices of $C_{2}\left(C_{1}^{\prime}\right)$ are the set of $v_{j}$ with $j$ even. The cycles $C_{1}, C_{2}$ are essential on $\hat{\Gamma}_{b}$.

Proof. Let $\varphi_{i}$ be the transition function of $\hat{e}_{i}$. Let $h$ be the number of orbits of $\varphi_{i}$. Since $\hat{e}_{i}$ has $n-1$ edges, all but one component of the subgraph of $\Gamma_{b}$ consisting of edges of $\hat{e}_{i}$ are cycles. Therefore $h-1 \leq 2$ by Lemma 2.14(2). Note also that each orbit contains the same number $(n / h)$ of vertices. Since $\Gamma_{a}$ has a Scharlemann bigon, $\hat{F}_{b}$ is separating and the number of positive vertices of $\Gamma_{b}$ is the same as that of negative vertices, hence the number of orbits $h$ is even, so we must have $h=2$. Hence $\hat{e}_{i}$ forms exactly one cycle component $C_{i}$ and one non-cycle component $C_{i}^{\prime}$ on $\Gamma_{b}$. Since each odd number appears twice 
at the endpoints of $\hat{e}_{1}, C_{1}$ contains $v_{j}$ with $j$ odd, and $C_{1}^{\prime}$ contains those with $j$ even. For the same reason the edges of $\hat{e}_{2}$ form a cycle $C_{2}$ and a chain $C_{2}^{\prime}$. Since $n / 2$ edges of $\hat{e}_{2}$ have even labels at $u_{1}, C_{2}$ must contain $v_{j}$ with $j$ even, while $C_{2}^{\prime}$ contains $v_{j}$ with $j$ odd. It follows that $C_{1} \cap C_{2}=\emptyset$.

When $w_{1}=n-2$ and $w_{2}=n$, the edges of $\hat{e}_{2}$ form exactly two cycles $C_{1}$ and $C_{2}$ on $\Gamma_{b}$, essential on $\hat{F}_{b}$, where the vertices of $C_{1}\left(C_{2}\right)$ are the $v_{j}$ with $j$ odd (even). This is because by Lemma 2.14(2) they cannot form more than two cycles, while $\Gamma_{b}$ being non-positive implies that $\hat{e}_{2}$ cannot form only one cycle. When $w_{1}=w_{2}=n-1$, let $C_{1}, C_{2}$ be the cycles given in Lemma 13.4. In either case, let $A_{1}, A_{2}$ be the annuli obtained by cutting $\hat{F}_{b}$ along $C_{1} \cup C_{2}$. Consider the cycles $\alpha=\alpha_{1} \cup \alpha_{2}$ and $\beta=\beta_{1} \cup \beta_{2}$ on $\Gamma_{b}$. Note that either $\alpha$ and $\beta$ are in different $A_{i}$, or each of them has exactly one edge in each $A_{i}$. We say that $\alpha, \beta$ are transverse to $C_{i}$ in the second case.

Lemma 13.5 The cycles $\alpha, \beta$ are disjoint, and transverse to $C_{i}$.

Proof. The first statement follows from Lemma 13.3(2), so we need only show that $\alpha, \beta$ are transverse to $C_{i}$.

First assume $\Delta=5$. Then $w_{5}=\frac{1}{2}\left(\Delta n-\left(w_{1}+\ldots+w_{4}\right)\right) \geq \frac{n}{2}+2$. By Lemma 2.3(3) we also have $w_{5} \leq \frac{n}{2}+2$, hence $w_{5}=\frac{n}{2}+2$, in which case the two outermost bigons of the family $\hat{e}_{5}$ are Scharlemann bigons, with label pair (12) and $(r+1, r+2)$, respectively, where $r=n / 2$. By Lemma 2.3(4) the label pair of $\beta_{1} \cup \beta_{2}$ must be either $(1,2)$ or $(r+1, r+2)$, and by Lemma 13.3 it cannot be the former. Therefore it must be $(r+1, r+2)$.

If $\alpha$ is not transverse to $C_{i}$, then it is an essential cycle in one of the annuli, say $A_{1}$, obtained by cutting $\hat{F}_{b}$ along $C_{1} \cup C_{2}$, so $\beta$ must be an essential cycle in the other annulus $A_{2}$. The two cycles $\alpha$ and $\beta$ separate the vertices of $C_{1}$ from $C_{2}$, except $v_{1}, v_{2}, v_{r+1}$ and $v_{r+2}$ which lie on $\alpha \cup \beta$. On the other hand, the edge $e$ in $\hat{e}_{5}$ adjacent to $\alpha_{2}$ has label pair $(3, n)$, so there is an edge on $\Gamma_{b}$ connecting $v_{3}$ to $v_{n}$. Since $n$ is even, the vertices $v_{3}, v_{n}$ belong to different $C_{i}$, but since $n>4$, neither 3 nor $n$ belongs to the set $\{1,2, r+1, r+2\}$, which is a contradiction.

Now assume $\Delta=4$. In this case the jumping number $J\left(r_{a}, r_{b}\right)= \pm 1$. Consider the two negative edges $e^{\prime}, e^{\prime \prime}$ of $\Gamma_{a}$ with label 2 at $u_{1}$. Note that their endpoints at $u_{1}$ are separated by the label 2 endpoints of $\alpha_{1}, \alpha_{2}$, hence by the Jumping Lemma, on $\Gamma_{b}$ the endpoints of $e^{\prime}, e^{\prime \prime}$ at $v_{2}$ are separated by those of $\alpha$; in other words, $e^{\prime}, e^{\prime \prime}$ are on different sides of the cycle $\alpha$. Assume that $v_{2} \in C_{2}$ is positive. If $\alpha$ is not transverse to $C_{2}$ then all positive edges at $v_{2}$ must be on one side of $\alpha$ because the other side is shielded by the cycle $C_{1}$, which contains only negative vertices. This is a contradiction. Therefore $\alpha$, and hence $\beta$, must be transverse to $C_{i}$.

Lemma 13.6 Each edge of $\hat{e}_{1} \cup \hat{e}_{2}$ is either on $C_{1} \cup C_{2}$ or parallel to an edge of $C_{1} \cup C_{2}$ on $\Gamma_{b}$. 
Proof. Let $C_{1}, C_{2}$ and $\alpha, \beta$ be as above. By definition $C_{2}$ consists of the edges in $\hat{e}_{2}$ with even labels. Let $C_{1}^{\prime}$ be the edges of $\hat{e}_{1}$ with even labels. Because of symmetry it suffices to show that each edge of $C_{1}^{\prime}$ is parallel to an edge in $C_{2}$.

Note that $\alpha \cap C_{2}=v_{2}$. Let $v_{t}=\beta \cap C_{2}$. (Thus $t$ is the even label of the Scharlemann bigon $\beta_{1} \cup \beta_{2}$ in $\Gamma_{a}$.) Since $w_{1}+w_{2}=2 n-2$ and the edges adjacent to $\hat{e}_{1} \cup \hat{e}_{2}$ on $\Gamma_{a}$ are the (12)-Scharlemann bigon $\alpha_{1} \cup \alpha_{2}$ and the $(t, t+1)$ - or $(t-1, t)$-Scharlemann bigon $\beta_{1} \cup \beta_{2}$, we see that $2, t$ are the only even labels appear three times among the endpoints of edges in $\hat{e}_{1} \cup \hat{e}_{2}$, hence on $\Gamma_{b}$ the edges of $C_{1}^{\prime}$ form a chain with endpoints at $v_{2}, v_{t}$, and possibly some cycle components. Therefore $C_{1}^{\prime}-v_{2} \cup v_{t}$ is disjoint from $\alpha \cup \beta \cup C_{1}$, hence lies in the interior of the two disks obtained by cutting $\hat{F}_{b}$ along $\alpha \cup \beta \cup C_{1}$. By Lemma 2.14(1) this implies that $C_{1}^{\prime}$ has no cycle component, and hence is a chain. Since $C_{1}^{\prime}$ contains all vertices of $C_{2}$, this also implies that one component of $C_{2}-v_{2} \cup v_{t}$ contains no vertices of $\Gamma_{b}$; in other words, the two vertices $v_{2}, v_{t}$ are adjacent on $C_{2}$.

Let $q, p$ be the transition number of $\hat{e}_{1}, \hat{e}_{2}$, respectively. Since $C_{2}$ has an edge connecting $v_{2}$ to $v_{t}$, we have $p \equiv \pm(t-2) \bmod n$. Since $C_{1}^{\prime}$ is a chain of length $(n / 2)-1$ connecting $v_{2}, v_{t}$, we have $((n / 2)-1) q \equiv \pm(t-2) \bmod n$. An edge of $C_{1}^{\prime}$ has even labels on both endpoints, so $q$ is even, hence $((n / 2)-1) q \equiv-q$ $\bmod n$. It follows that $p \equiv \pm q \bmod n$, which implies that each edge $e^{\prime}$ of $C_{1}^{\prime}$ has its endpoints on adjacent vertices of $C_{2}$. Let $e$ be the edge of $C_{2}$ connecting these two vertices. Since $e^{\prime}$ has interior disjoint from $\alpha \cup \beta \cup C_{1}$, it must be parallel to $e$.

Proposition 13.7 The case that $\Gamma_{a}, \Gamma_{b}$ are non-positive, $n_{a}=2, n=n_{b}>4$, and $w_{3}+w_{4} \leq w_{1}+w_{2}=2 n-2$, is impossible.

Proof. First assume that $w_{1}=n-2$ and $w_{2}=n$. By Lemma 13.3, the label pair of $\beta$ is $(k, k+1)$, where $k$ is odd and $k \neq 1$. If $k=n-1$ then the edges of $\hat{e}_{2}$ are co-loops, which contradicts Lemma 2.14(2). Therefore $n-3 \geq k \geq 3$.

Since the label sequence of $\hat{e}_{1}$ at $u_{2}$ is $k+2, \ldots, n, 1, \ldots, k-1$, the above implies that there are adjacent edges $e_{1}^{\prime}, e_{n}^{\prime} \in \hat{e}_{1}$ with labels 1 and $n$ at $u_{2}$, respectively. By Lemma 13.6 each edge of $\hat{e}_{1}$ is parallel to some edge of $\hat{e}_{2}$ on $\Gamma_{b}$, hence the transition function $\psi_{1}$ of $\hat{e}_{1}$ is either equal to $\psi_{2}$ of $\hat{e}_{2}$, or $\psi_{2}^{-1}$, but since the two edges of $\hat{e}_{1} \cup \hat{e}_{2}$ with label $n$ at $u_{1}$ have labels $k+1$ and $k-1$ respectively at $u_{2}$, the first case is impossible, hence $\psi_{1}=\psi_{2}^{-1}$. Let $\hat{e}_{2}=e_{1} \cup \ldots \cup e_{n}$, where $e_{i}$ has label $i$ at $u_{1}$. Since $e_{1}$ is the only edge of $\hat{e}_{2}$ with label 1 at $u_{1}$, it must be the one that is parallel to $e_{1}^{\prime}$ on $\Gamma_{b}$. Similarly, $e_{n}$ is parallel to $e_{n}^{\prime}$ on $\Gamma_{b}$. This is a contradiction to Lemma 2.19 .

Now assume that $w_{1}=w_{2}=n-1$. As above, let $\hat{e}_{2}=e_{2} \cup \ldots \cup e_{n}$, where $e_{i}$ has label $i$ at $u_{1}$. The label sequence of $\hat{e}_{1}$ at $u_{2}$ is $k+1, k+2, \ldots, n, 1, \ldots, k-1$. By Lemma 13.3, $k$ is even, and $\{1,2\} \cap\{k, k+1\}=\emptyset$, so $n-2 \geq k \geq 4$. It follows that there are three consecutive edges $e_{n}^{\prime}, e_{1}^{\prime}, e_{2}^{\prime}$ of $\hat{e}_{1}$ such that $e_{i}^{\prime}$ has label $i$ at $u_{2}$. For the same reason as above, $e_{2}^{\prime}$ is parallel to $e_{2}$ and $e_{n}^{\prime}$ is parallel to $e_{n}$ on $\Gamma_{b}$. Since the number of edges between $e_{n}^{\prime}$ and $e_{2}^{\prime}$ is 1 while the number of edges between $e_{2}$ and $e_{n}$ is $n-3>1$ on $\Gamma_{a}$, this is a contradiction to Lemma 2.19 . 


\section{The case $n_{a}=2, n_{b}>4, \Gamma_{1}, \Gamma_{2}$ non-positive, and $w_{1}=w_{2}=n_{b}$}

In this section we consider the case that $n_{a}=2, n=n_{b}>4, \Gamma_{1}, \Gamma_{2}$ non-positive, and $w_{1}=w_{2}=n$. We will also assume without loss of generality that $w_{3} \geq w_{4}$. Let $\hat{e}_{1}=e_{1} \cup \ldots \cup e_{n}, \hat{e}_{2}=e_{1}^{\prime} \cup \ldots \cup e_{n}^{\prime}$, and assume that $e_{i}, e_{i}^{\prime}$ have label $i$ at $u_{1}$.

Let $r$ be such that the label of the endpoint of $e_{1}$ on $\partial u_{2}$ is $r+1$. One can check that both $e_{i}, e_{i}^{\prime}$ have label $r+i$ at $\partial u_{2}$.

Since $\Gamma_{b}$ is non-positive, the vertices of $\Gamma_{b}$ cannot all be parallel, so the edges of $\hat{e}_{1}$ form at least two cycles on $\Gamma_{b}$. By Lemma 2.14(2) they form exactly two cycles $C_{1} \cup C_{2}$ on $\Gamma_{b}$.

Lemma 14.1 $\Gamma_{a}$ is not kleinian. In particular, $\Gamma_{b}$ cannot contain four parallel positive edges.

Proof. If $\Gamma_{a}$ is kleinian then by Lemma 6.2(4) there is a free orientation reversing involution $\phi$ of $\left(\hat{F}_{a}, \Gamma_{a}\right)$, which maps $u_{1}$ to $u_{2}$, and is label preserving. If there is no loop on $\Gamma_{a}$ (i.e. $w_{5}=w_{6}=0$ ), then $\Delta=4$ and $w_{i}=n$ for all $i$, so the label sequences of $\hat{e}_{i}$ at $u_{1}$ are all the same. The above implies that the label sequences of $\hat{e}_{i}$ at $u_{2}$ are also the same as those at $u_{1}$, so the transition function $\varphi$ defined by $\hat{e}_{i}$ is the identity map and hence all edges of $\Gamma_{a}$ are co-loops, contradicting the 3-Cycle Lemma 2.14(2).

Now assume $w_{5}=w_{6}>0$. Then $\phi$ maps $\hat{e}_{1} \cup \hat{e}_{2}$ to either $\hat{e}_{1} \cup \hat{e}_{2}$ or $\hat{e}_{3} \cup \hat{e}_{4}$. In the first case since $\phi$ is label preserving and orientation reversing on the torus, the label sequence of $\hat{e}_{1}$ at $u_{2}$ is the same as that of $\hat{e}_{1}$ at $u_{1}$, hence all edges of $\hat{e}_{1}$ are co-loops and we have a contradiction to Lemma 2.14(2). In the second case $w_{3}=w_{4}=w_{1}=w_{2}=n$, so $\Delta=5$ and $w_{5}=w_{6}=n / 2$. We have assumed that $\hat{e}_{1}$ has label sequence $1,2, \ldots, n$ at $u_{1}$. so $\hat{e}_{3}$ has the same label sequence at $u_{2}$. Since $w_{5}=n / 2$, the label sequence of $\hat{e}_{1}$ at $u_{2}$ is $(k+1, k+2, \ldots n, 1, \ldots, k)$, where $k=n / 2$. Therefore $\phi$ is of period 2 , so it has $n / 2>2$ orbits, which again contradicts Lemma 2.14(2).

The second statement follows from the above and Lemma 13.2(2).

Lemma 14.2 The edges $e_{i}, e_{i}^{\prime}$ are parallel on $\Gamma_{b}$.

Proof. The cycles $C_{1} \cup C_{2}$ defined at the beginning of the section cut the torus $\hat{F}_{b}$ into two annuli $A_{1}, A_{2}$. Each $e_{i}^{\prime}$ lies in one of the $A_{j}$ and has the same endpoints as $e_{i}$, so if it is not parallel to $e_{i}$ and $e_{i} \subset C_{1}$ then it is parallel to $C_{1}-e_{i}$. There are at most two such $e_{i}^{\prime}$ for $C_{1}$, one in each $A_{j}$. Since $n>4, C_{1}$ contains at least three edges, hence there exists some $e_{j}^{\prime}$ parallel to $e_{j} \subset C_{1}$.

Assume $e_{i}$ is not parallel to $e_{i}^{\prime}$, and let $e_{j}, e_{j}^{\prime}$ be parallel on $\hat{F}_{b}$, which exist by the above. Let $D$ (resp. $D^{\prime}$ ) be the disk on $F_{a}$ that realizes the parallelism between $e_{i}, e_{j}$ (resp. $e_{i}^{\prime}, e_{j}^{\prime}$ ), and let $D^{\prime \prime}$ be the disk between $e_{j}$ and $e_{j}^{\prime}$ on $F_{b}$. Shrinking $V_{b}$ to its core $K_{b}, B=D \cup D^{\prime} \cup D^{\prime \prime}$ becomes a disk in $M\left(r_{b}\right)$ with $\partial B=e_{i} \cup e_{i}^{\prime}$, which contradicts the fact that $\hat{F}_{b}$ is incompressible in $M\left(r_{b}\right)$. 


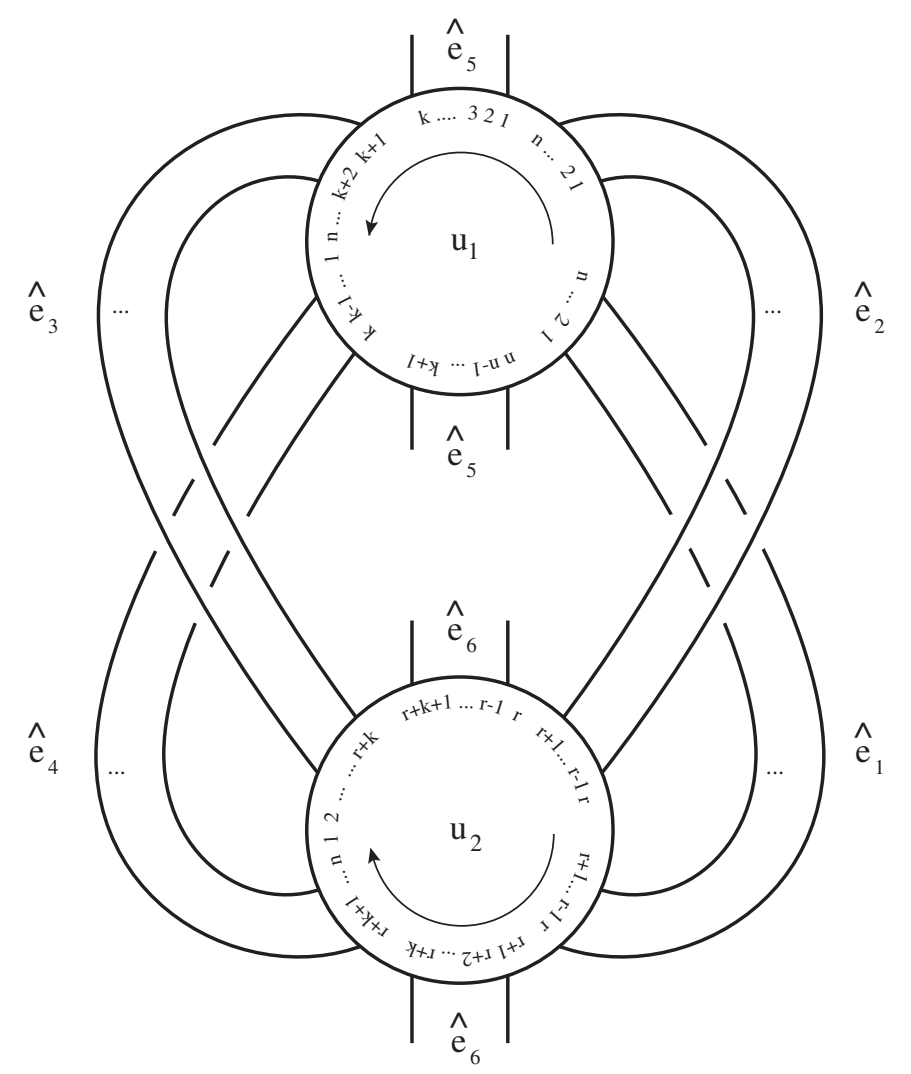

Figure 14.1

Lemma $14.3 w_{3}+w_{4} \neq n$.

Proof. Assume to the contrary that $w_{3}+w_{4}=n$. We have $\Delta=4$ as otherwise there would be $n$ parallel positive edges in $\hat{e}_{5}$, contradicting Lemma 2.3(3). Now $w_{5}=w_{6}=n / 2$, so the graph $\Gamma_{a}$ is as shown in Figure 14.1, where $k=n / 2$. Since $\Delta=4$, we may assume that the jumping number is 1 .

Let $i$ be a label such that $1 \leq i \leq k$, so it appears on the top of the vertex $u_{1}$ in Figure 14.1. Consider the vertex $v_{i}$ of $\Gamma_{b}$, see Figure 14.2. By Lemma 14.2 $e_{i}$ of $\hat{e}_{1}$ is parallel to $e_{i}^{\prime}$ of $\hat{e}_{2}$. Since $e_{i}$ and $e_{i}^{\prime}$ have the same label 1 at $v_{i}$, there is an edge of $\hat{e}_{3} \cup \hat{e}_{4}$ between them. Similarly there are parallel edges $e_{j}, e_{j}^{\prime}$ with label 2 at $v_{i}$, and there is another edge between them. See Figure 14.2. From the labeling we see that the two negative edges at $v_{i}$ (corresponding to loops in $\Gamma_{a}$ ) must be adjacent to each other. 


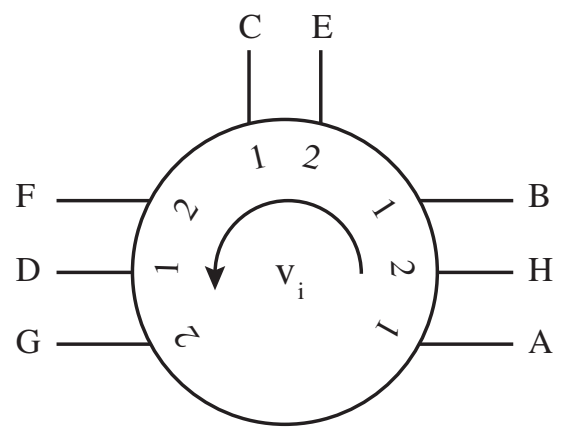

Figure 14.2

On $\partial u_{1}$ the $i$-labels appear on endpoints of edges in the order of $A, B, C, D$, where $A \in e_{i}, B \in e_{i}^{\prime}, C$ is a loop in $\hat{e}_{5}$, and $D \in \hat{e}_{3} \cup \hat{e}_{4}$. Since the jumping number is 1, the 1-labels at $v_{i}$ also appear in the same order. In Figure 14.2 this implies that the negative edge $C$ appears on the top of the vertex.

Now consider the four edges labeled 2 at $v_{i}$, denoted by $E, F, G, H$, where $E$ is the negative edge, which is uniquely determined. Since $F, G$ are parallel positive edges on $\Gamma_{b}$, they are the $e_{j}, e_{j}^{\prime}$ given above, belonging to $\hat{e}_{1} \cup \hat{e}_{2}$. On $\partial u_{2}$ this implies that the endpoint of the loop $E$ labeled $i$ appears on the top of $\partial u_{2}$ in Figure 14.1. Since $i$ is any label between 1 and $n / 2$, it follows that the labels on the top of $\partial u_{2}$ must be $1,2, \ldots, k$, so the integer $r$ in the figure satisfies $r=k$. However, in this case the edges of $\hat{e}_{1}$ would form cycles of length 2 in $\Gamma_{b}$, which is a contradiction to Lemma 2.14(2).

Lemma $14.4 w_{3}=n$, and $0<w_{4}<n$. Moreover, an edge $e^{\prime \prime}$ of $\hat{e}_{3}$ with label $j$ at $u_{2}$ is parallel to the edges $e_{j}$ and $e_{j}^{\prime}$.

Proof. We have assumed $w_{4} \leq w_{3} \leq n$. If $w_{4}=n$ then the argument of Lemma 14.2 applied to $\hat{e}_{3}, \hat{e}_{4}$ shows that each edge of $\hat{e}_{3}$ is parallel to exactly one edge of $\hat{e}_{4}$. On the other hand, since the two parallel edges $e_{i}, e_{i}^{\prime}$ in the proof of Lemma 14.2 have the same label 1 at the vertex $v_{i}$, there must be another edge $e_{i}^{\prime \prime}$ in $\hat{e}_{3} \cup \hat{e}_{4}$ between $e_{i}$ and $e_{i}^{\prime}$. Together with the other edge in $\hat{e}_{3} \cup \hat{e}_{4}$ which is parallel to $e_{i}^{\prime \prime}$, we get four parallel positive edges in $\Gamma_{b}$, which contradicts Lemma 14.1. Therefore $w_{4}<n$.

Recall from Lemma 14.2 that the edges $e_{i}$ and $e_{i}^{\prime}$ are parallel in $\Gamma_{b}$, with the same label 1 at $u_{i}$, so there must be another edge $e_{i}^{\prime \prime} \in \hat{e}_{3} \cup \hat{e}_{4}$ between them. Note also that if $e_{i}$ has label $i+r$ at $u_{2}$ then $e_{i}^{\prime \prime}$ has the property that it has label $i$ at $u_{2}$ and $i+r$ at $u_{1}$. This is true for all $i$, so either $\hat{e}_{3}$ and $\hat{e}_{4}$ have the same transition function, or these $e_{i}^{\prime \prime}$ all belong to the same family. The first case happens only if $w_{3}+w_{4} \equiv 0 \bmod n$, which is impossible because by Lemma 14.3 we have $w_{3}+w_{4} \neq n$, while $w_{3} \leq n$ and by the above we have $w_{4}<n$. Therefore all the $e_{i}^{\prime \prime}$ belong to $\hat{e}_{3}$. Since $w_{3} \leq n$, this implies that $w_{3}=n$. Again by Lemma 14.3 we have $w_{4} \neq 0$. 


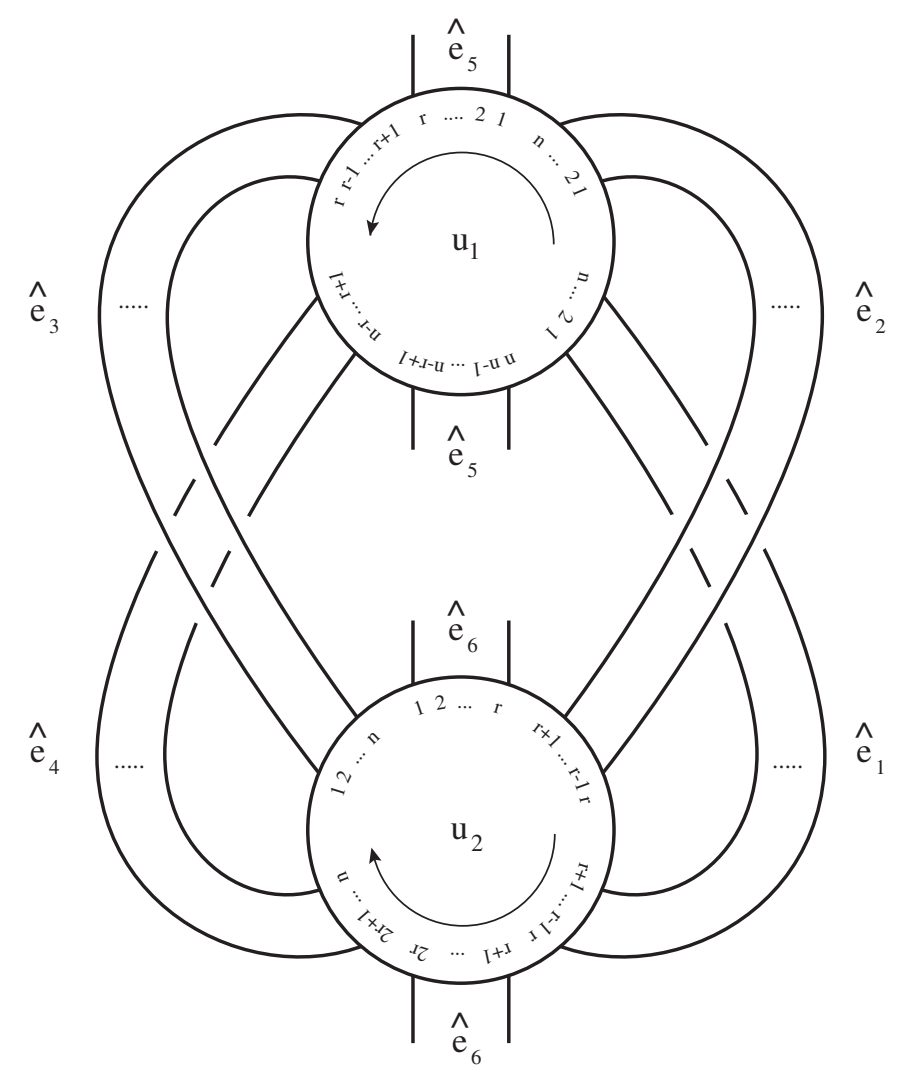

Figure 14.3

Lemma 14.5 The label sequence of $\hat{e}_{3}$ is $1,2, \ldots, n$ at $u_{2}$, and $1+r, 2+r, \ldots, n, 1, \ldots, r$ at $u_{1}$. The labels of $\Gamma_{a}$ are as shown in Figure 14.3.

Proof. First assume that the label sequence of $\hat{e}_{3}$ at $u_{2}$ is not $1,2, \ldots, n$. Then there is a pair of adjacent parallel edges $e_{n}^{\prime \prime}, e_{1}^{\prime \prime}$ with label $n$ and 1 at $u_{2}$, respectively. By Lemma $14.4 e_{1} \cup e_{1}^{\prime \prime}$ and $e_{n} \cup e_{n}^{\prime \prime}$ are parallel pairs on $\Gamma_{b}$. Since $e_{1}^{\prime \prime}, e_{n}^{\prime \prime}$ are adjacent on $\Gamma_{a}$ while $e_{1}, e_{n}$ are not, this is a contradiction to Lemma 2.19. Therefore the label sequence of $\hat{e}_{3}$ at $u_{2}$ must be $1,2, \ldots, n$.

Since by Lemma 14.4 the edge $e_{i}^{\prime \prime}$ connects $v_{i}$ to $v_{i+r}$ with label 2 at $v_{i}$ and 1 at $v_{i+r}$, we see that on $\Gamma_{a}$ it has label $i$ at $u_{2}$ and $i+r$ at $u_{1}$, hence the label sequence of $\hat{e}_{3}$ at $u_{1}$ is $r+1, \ldots, n, 1, \ldots, r$. The labels of $\hat{e}_{1}, \hat{e}_{2}, \hat{e}_{3}$ determine those of the loops, and hence those of $\hat{e}_{4}$. Therefore $\Gamma_{a}$ must be as shown in Figure 14.3 .

Lemma 14.6 (1) The jumping number $J= \pm 1$. 
(2) Orient the negative edges of $\Gamma_{a}$ from $u_{1}$ to $u_{2}$. Then on $\Gamma_{b}$ the edges of $\hat{e}_{1}$ form two essential cycles of opposite orientation on $\hat{F}_{b}$.

Proof. (1) Since $\Delta=4$ or 5 , the jumping number is either \pm 1 or \pm 2 . Let $e_{i}, e_{i}^{\prime}$ be the edges of $\hat{e}_{1}, \hat{e}_{2}$, respectively, with label $i$ at $u_{1}$. If $J= \pm 2$ then these edges are not adjacent among the 1-edges at $v_{i}$ in $\Gamma_{b}$. Since by Lemma 14.2 they are parallel in $\Gamma_{b}$, there would be more than $2 n_{a}=4$ parallel edges in $\Gamma_{b}$, which contradicts Lemma 2.2(2). Therefore $J= \pm 1$. Changing the orientation of $\hat{F}_{b}$ if necessary, we may assume that $J=1$.

(2) Now let $C_{1}, C_{2}$ be the cycles on $\Gamma_{b}$ consisting of edges of $\hat{e}_{1}$. We need to show that they are of opposite orientation.

Let $a_{1}, a_{2}, \bar{a}, a_{3}$ be the edges with label 1 at $u_{1}$, where $a_{i} \in \hat{e}_{i}$ for $i=1,2,3$, and $\bar{a} \in \hat{e}_{5}$. Note that they appear in this order on $\partial u_{1}$. Since $J=1$, they also appear in this order on $\partial v_{1}$ in $\Gamma_{b}$, see Figure 14.4. By the proof of Lemma 14.3 we see that $a_{3}$ is in the middle of a pair of parallel positive edges incident to $v_{1}$, which is not parallel to $a_{1}, a_{2}$, hence the orientation of $C_{1}$ must be as shown in Figure 14.4, where $C_{1}$ is represented by the lower level chain.

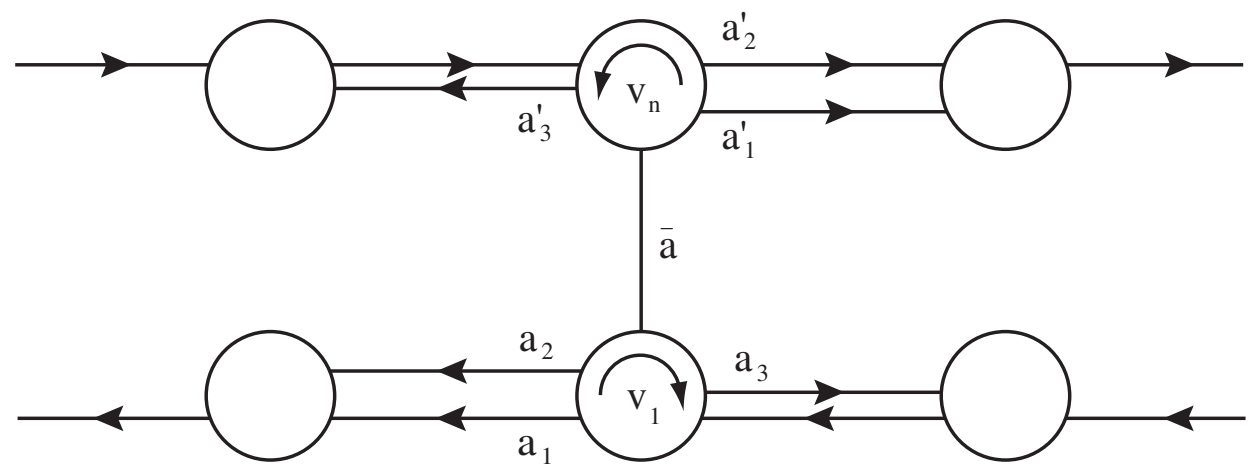

Figure 14.4

Now consider the edges labeled $n$ at $u_{1}$. There are 5 of them if $\Delta=5$, but we only consider $\bar{a}$ and the edges $a_{1}^{\prime}, a_{2}^{\prime}, a_{3}^{\prime}$, where $a_{i}^{\prime} \in \hat{e}_{i}$. The order of the label $n$ endpoints of these edges on $\partial u_{1}$ is $a_{3}^{\prime}, \bar{a}, a_{1}^{\prime}, a_{2}^{\prime}$, while the orientation of $v_{n}$ is opposite to that of $v_{1}$. Therefore these edges appear on $\partial v_{n}$ as shown in Figure 14.4. We see that $C_{1}, C_{2}$ are of opposite orientation on $\hat{F}_{b}$. 


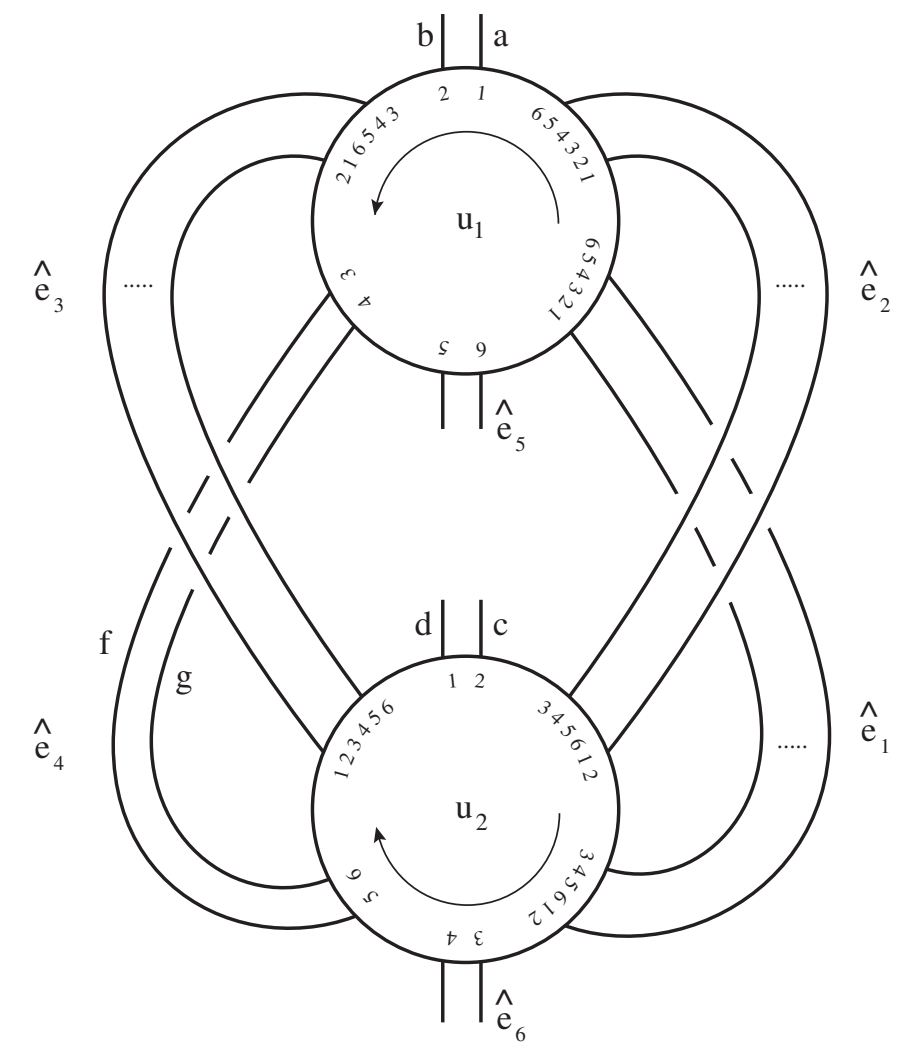

(a)

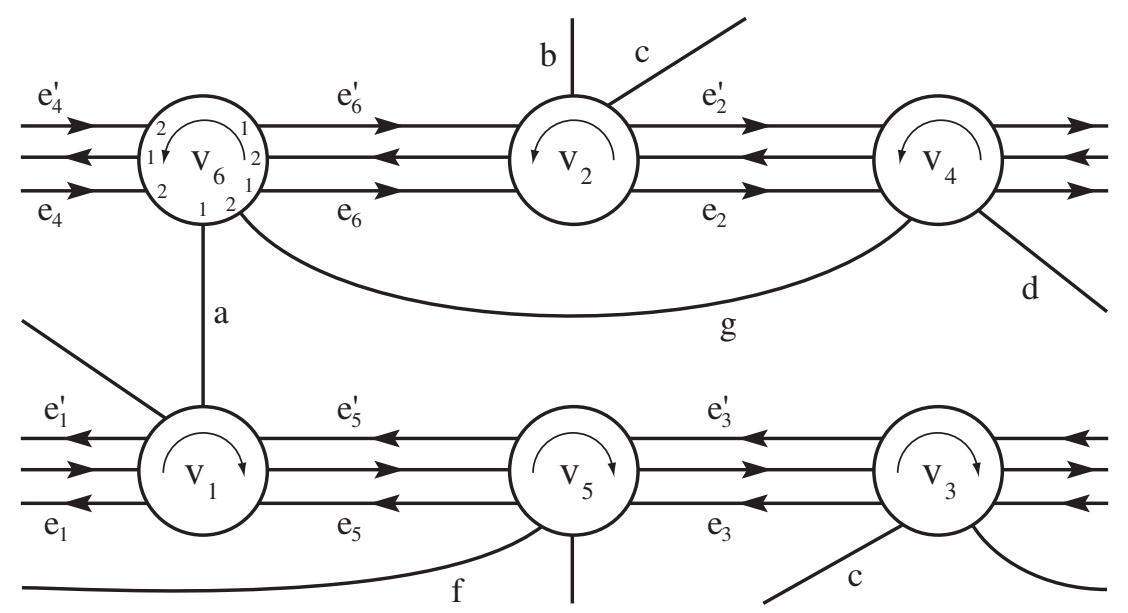

(b) 
Figure 14.5

Proposition 14.7 Suppose $n_{a}=2, n>4, \Gamma_{a}, \Gamma_{b}$ are non-positive, and $w_{1}=$ $w_{2}=n$.

(1) On $\Gamma_{b}$ each edge of $\hat{e}_{4}$ connects a pair of adjacent vertices of some $C_{i}$, but is not parallel to an edge of $C_{i}$.

(2) Two adjacent edges of $\hat{e}_{4}$ lie in different annuli of $\hat{F}_{b}-\cup C_{i}$.

(3) $w_{4}=w_{5}=w_{6}=2, \Delta=4$, and $n=6$.

(4) The graphs $\Gamma_{a}, \Gamma_{b}$ and their edge correspondence are as shown in Figure 14.5, where $e_{i}$ (resp. $e_{i}^{\prime}$ ) is the edge in $\hat{e}_{1}$ (resp. $\hat{e}_{2}$ ) with label $i$ at $u_{1}$, and the edge between $e_{i}, e_{i}^{\prime}$ is the edge of $\hat{e}_{3}$ with label $i$ at $u_{2}$.

Proof. (1) From Figure 14.3 we see that an edge $e$ of $\hat{e}_{4}$ with label $i$ at $u_{1}$ has label $i+r$ at $u_{2}$. Since the transition function of $\hat{e}_{1}$ also maps $i$ to $i+r, v_{i}$ and $v_{i+r}$ are connected by the edge $e_{i}$ of $\hat{e}_{1}$, and hence are adjacent on one of the cycles $C_{j}$. This proves the first part of (1). By Lemmas 14.2 and 14.4 each edge $e_{i}$ of $C_{j}$ is parallel to an edge $e_{i}^{\prime}$ in $\hat{e}_{2}$ and an edge $e_{i}^{\prime \prime}$ in $\hat{e}_{3}$, so if $e$ is parallel to $e_{i}$ then there would be four parallel positive edges in $\Gamma_{b}$, which would contradict Lemma 14.1.

(2) By Lemma 14.6(2) the two cycles $C_{1}, C_{2}$ have opposite orientations. Without loss of generality we may assume that the orientations of $C_{i}$ are as shown in Figure 14.5. Recall that $C_{1}$ is the cycle containing the vertex $v_{1}$. By Lemma 14.6(1) we may assume without loss of generality that the jumping number of the graphs is 1 . Let $e$ be an edge of $\hat{e}_{4}$ with label $k$ at $u_{1}$, and let $e_{k}, e_{k}^{\prime}, e_{k}^{\prime \prime}$ be the edges of $\hat{e}_{1}, \hat{e}_{2}, \hat{e}_{3}$ with label $k$ at $u_{1}$. Then the endpoints of these edges appear at $\partial u_{1}$ in the order $e_{k}, e_{k}^{\prime}, e_{k}^{\prime \prime}, e$, so on $\partial v_{k}$ they appear in the same order. If $v_{k}$ is in $C_{1}$ then the orientation of $C_{1}$ points to the left and the orientation of $v_{k}$ is clockwise, so $e$ is in the annulus below $C_{1}$. If $v_{k}$ is in $C_{2}$ then the orientation of $C_{1}$ points to the right and the orientation of $v_{k}$ is counterclockwise, so again $e$ is in the annulus below $C_{1}$. Since the labels of adjacent edges of $\hat{e}_{4}$ belong to different $C_{i}$ in $\Gamma_{b}$, it follows from the above that they are in different annuli of $\hat{F}_{b}-\cup C_{i}$.

(3) Since each $C_{i}$ contains $n / 2>2$ vertices, there cannot be two edges on the same side of $C_{i}$ connecting two different pairs of adjacent vertices and yet not parallel to an edge of $C_{i}$. Hence by (1) and (2) $\hat{e}_{4}$ contains at most two edges. By Lemma $14.4 w_{4}>0$, and from the labeling in Figure 14.3 we see that $w_{4}$ is even. Therefore $w_{4}=2$.

If $\Delta=5$ then the loop family of $\Gamma_{a}$ at $u_{1}$ contains $n-1$ edges. This contradicts Lemma 2.3(3) for $n>4$. Hence $\Delta=4$.

Let $e$ be an edge of $\hat{e}_{4}$ with endpoints on $v_{i}$ and $v_{j}$ in $C_{2}$, lying on the annulus $A$ below $C_{2}$. By (1) it is not parallel to the edge on $C_{2}$ connecting $v_{i}, v_{j}$, so on $A$ it separates $C_{1}$ from other vertices of $C_{2}$, hence there is no edge in $A$ connecting $C_{1}$ to vertices of $C_{2}$ except possibly $v_{i}$ and $v_{j}$. By Lemmas 14.2 and 14.4 there are three parallel edges for each edge of $C_{i}$. Together with $e$, they contribute 7 edge endpoints to each of $v_{i}$ and $v_{j}$, therefore $\Delta=4$ implies that there are 
at most two edges in $A$ connecting $C_{1}$ to $C_{2}$, one for each of $v_{i}, v_{j}$. Note that these correspond to loop edges in $\Gamma_{a}$. Therefore the two annuli give rise to at most 4 loops in $\Gamma_{a}$, so $w_{5}=w_{6} \leq 2$. Since $n>4$ and $2 w_{5}+w_{4}=(\Delta-3) n=n$, it follows that $n=6$, and $w_{5}=w_{6}=2$.

(4) By Lemma 14.5 $\Gamma_{a}$ is the graph in Figure 14.3. We have $w_{4}=w_{5}=$ $w_{6}=2$ and $w_{1}=w_{2}=w_{3}=n=6$, hence $\Gamma_{a}$ is as shown in Figure 14.5(a).

The edges in $\hat{e}_{2}, \hat{e}_{3}$ are parallel to those in $\hat{e}_{1}$, as shown in Lemmas 14.2 and 14.5, therefore they form families of three parallel edges, as shown in Figure 14.5(b). Orientations are from $u_{1}$ to $u_{2}$ on $\Gamma_{a}$, so the tails of these edges are labeled 1 and the heads labeled 2 on $\Gamma_{b}$. The two edges in $\hat{e}_{4}$ connect $v_{4}, v_{6}$ and $v_{3}, v_{5}$ respectively, and by $(1)$ and (2) they are not parallel to edges in $C_{i}$ and lie in different annuli of $\hat{F}_{b}-C_{1} \cup C_{2}$, hence we may assume that they look like that in Figure 14.5(b). The four edges in $\hat{e}_{5}$ and $\hat{e}_{6}$ are now determined by the labeling of the edges and the vertices on $\Gamma_{b}$. The labeling of the weight 3 families in $\Gamma_{b}$ are determined by the single edges and the assumption that the jumping number is 1 .

\section{$15 \Gamma_{a}$ with $n_{a} \leq 2$}

The next few sections deal with the case that $n_{a} \leq 2$ and $n_{b} \leq 4$. In this section we set up notation and give some preliminary results.

We use $G=\left(b_{1}, b_{2}, b_{3}\right)$ to denote a graph $G$ on a torus with one vertex and three families of edges weighted $b_{1}, b_{2}, b_{3}$. Similarly, denote by $G=\left(\rho ; a_{1}, \ldots, a_{4}\right)$ a graph $G$ on a torus which has two vertices, two families of loops of weight $\rho$, and four families of edges $\hat{e}_{i}$ with weight sequence $a_{1}, \ldots, a_{4}$ around the vertices. It is possible that $\rho$ and some of the $a_{i}$ may be zero. When $\rho=0$ we will simply write $G=\left(a_{1}, \ldots, a_{4}\right)$. Note that the weight sequence is defined up to cyclic rotation and reversal of order. When $\rho=0$, any weight 0 can be moved around without changing the graph, hence $(2,2,0,0)$ is equivalent to $(2,0,2,0)$, but $(1 ; 2,0,2,0)$ is different from $(1 ; 2,2,0,0)$ and $(3,1,3,1)$ is different from $(3,3,1,1)$. When it is necessary to indicate whether the vertices of $G$ are parallel or antiparallel, we write $G=+\left(\rho ; a_{1}, \ldots, a_{4}\right)$ if the vertices of $G$ are parallel, and $G=-\left(\rho ; a_{1}, \ldots, a_{4}\right)$ otherwise.

If $n_{a}=2$ then $\Gamma_{a}$ is of the form $\left(\rho ; a_{1}, \ldots, a_{4}\right)$. Note that if $e$ is co-loop then all edges parallel to $e$ are. Hence we may define an edge $\hat{e}$ in $\hat{\Gamma}_{a}$ to be co-loop if one (and hence all) of its edges is co-loop. Define $\epsilon_{i}=0$ if $\hat{e}_{i}$ is a co-loop, and $\epsilon_{i}=1$ otherwise. Note that if $n_{b}=2$ then $\epsilon_{i}$ measures the difference between the labels at the two endpoints of an edge in $\hat{e}_{i}$, so it is actually the same as the transition number defined in Section 2.

Lemma 15.1 (The Congruence Lemma.) Suppose $n_{a}=2$. Let $\hat{e}_{i}, \hat{e}_{j}$ be edges in $\hat{\Gamma}_{a}$ with the endpoints on the same pair of vertices $u_{1}, u_{2}$. Let $a_{k}$ be the weight of $\hat{e}_{k}$.

(1) If $\hat{\Gamma}_{a}$ has no loops and $a_{i}, a_{j} \neq 0$ then $a_{i}+\epsilon_{i} \equiv a_{j}+\epsilon_{j} \bmod 2$. In other words, $a_{i} \equiv a_{j} \bmod 2$ if and only if $\hat{e}_{i}$ and $\hat{e}_{j}$ are both co-loop or both non 
co-loop.

(2) If $\hat{\Gamma}_{a}$ has loops and $a_{i}, a_{j} \neq 0$, then $a_{i} \equiv a_{j} \bmod 2$.

(3) If $\hat{\Gamma}_{a}$ has loops and the endpoints of $\hat{e}_{i}, \hat{e}_{j}$ at $u_{1}$ are on the same side of the loop at $u_{1}$ then $a_{i} \equiv a_{j} \bmod 2$.

Proof. (1) Delete edges of $\hat{\Gamma}_{a}$ with zero weight. We need only prove the statement for adjacent edges $\hat{e}_{1}, \hat{e}_{2}$ of $\hat{\Gamma}_{a}$ with non-zero weight. Let $e_{1}, \ldots, e_{a_{1}}$ and $e_{1}^{\prime}, \ldots, e_{a_{2}}^{\prime}$ be the edges in $\hat{e}_{1}$ and $\hat{e}_{2}$, respectively, so that $e_{1}^{\prime}$ is adjacent to $e_{a_{1}}$ on $\partial u_{1}$. Then $e_{a_{2}}^{\prime}$ is adjacent to $e_{1}$ on $\partial u_{2}$. Without loss of generality assume that the label of $e_{i}$ at $u_{1}$ is $i$. (Since $n_{b}=2$, all labels of endpoints of $e_{i}, e_{j}^{\prime}$ are mod 2 integers.) Then the label of $e_{1}$ at $u_{2}$ is $1+\epsilon_{1}$. On the other hand, the label of $e_{1}^{\prime}$ at $u_{1}$ is $a_{1}+1$, so the label of $e_{a_{2}}^{\prime}$ at $u_{1}$ is $a_{1}+a_{2}$, and the label of $e_{a_{2}}^{\prime}$ at $u_{2}$ is $a_{1}+a_{2}+\epsilon_{2}$. See Figure 15.1. Since $e_{a_{2}}^{\prime}$ is adjacent to $e_{1}$ on $u_{2}$, the label of $e_{1}$ on $u_{2}$ is $a_{1}+a_{2}+\epsilon_{2}+1$. These two equations give

$$
1+\epsilon_{1} \equiv a_{1}+a_{2}+\epsilon_{2}+1 \quad \bmod 2
$$

It follows that $a_{1} \equiv a_{2}$ if and only if $\epsilon_{1} \equiv \epsilon_{2} \bmod 2$.

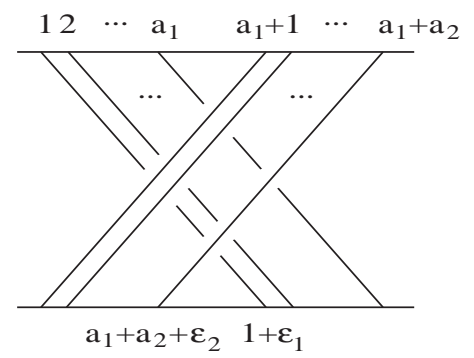

Figure 15.1

(2) Again we need only prove the statement for adjacent edges $\hat{e}_{1}, \hat{e}_{2}$ with non-zero weight. Since $\Gamma_{a}$ has loops, the two vertices of $\hat{\Gamma}_{b}$ must be antiparallel. If the two vertices of $\Gamma_{a}$ are parallel then $\hat{e}_{1}, \hat{e}_{2}$ are both positive on $\Gamma_{a}$ and hence both negative on $\Gamma_{b}$, so they are both non co-loops. Similarly if the two vertices of $\Gamma_{a}$ are antiparallel then $\hat{e}_{1}, \hat{e}_{2}$ are both co-loops. Therefore $\epsilon_{1}=\epsilon_{2}$ in either case. Note that the endpoints of $\hat{e}_{1}, \hat{e}_{2}$ are on the same side of the loop at $u_{1}$ if and only if their other endpoints are on the same side of the loop at $u_{2}$. Since the number of loops at the two vertices are the same, the distance between the endpoints of $e_{a_{1}}$ and $e_{1}^{\prime}$ on $\partial u_{1}$ is the same as that of $e_{a_{2}}^{\prime}$ and $e_{1}$ on $\partial u_{2}$, hence the above argument can be modified to show that if $a_{1}, a_{2} \neq 0$ then $a_{1} \equiv a_{2}$ mod 2. More explicitly, if there is no loop between the endpoints of $\hat{e}_{1}, \hat{e}_{2}$ then the above argument follows verbatim, while if there are $k$ loops between them then the endpoint $e_{a_{2}}^{\prime}$ is $a_{1}+a_{2}+k$ at $u_{1}$, and $a_{1}+a_{2}+k+\epsilon_{2}$ at $u_{2}$, and we have $a_{1}+a_{2}+k+\epsilon_{2}+k+1=1+\epsilon_{1}$, hence the result follows because $\epsilon_{1}=\epsilon_{2} \bmod 2$. 
(3) This follows from (2) if $a_{i}, a_{j} \neq 0$. If $a_{i}=0$ and $\hat{e}_{j}$ is on the same side of the loop as $\hat{e}_{1}$ (which is empty), then since a loop has different labels on its two endpoints, the number of edges in $\hat{e}_{2}$ must be even, hence $a_{2} \equiv a_{1}=0 \bmod$ 2 .

Lemma 15.2 Suppose $\Gamma_{b}$ is positive, and contains a black bigon $e_{1} \cup e_{2}$ and a white bigon $e_{1}^{\prime} \cup e_{2}^{\prime}$. Then on $\Gamma_{a}$ the four edges $e_{1}, e_{2}, e_{1}^{\prime}, e_{2}^{\prime}$ cannot be contained in two families of parallel edges.

Proof. Recall that no two edges are parallel on both graphs, so if the lemma is not true then we may assume that $e_{i}$ is parallel to $e_{i}^{\prime}$ on $\Gamma_{a}$. Let $B_{i}$ be the disk on $F_{a}$ realizing the parallelism, and let $D, D^{\prime}$ be the bigon on $F_{b}$ bounded by $e_{1} \cup e_{2}$ and $e_{1}^{\prime} \cup e_{2}^{\prime}$, respectively. Then $A=D \cup D^{\prime} \cup B_{1} \cup B_{2}$ is either a Möbius band or an annulus. The first case contradicts the fact that a hyperbolic manifold $M$ contains no Möbius bands. In the second case $A$ contains a single white bigon and hence each of its boundary components intersects a curve of slope $r_{a}$ transversely at a single point. Since $e_{1}$ is an essential arc on both $F_{a}$ and $A, A$ cannot be boundary parallel, and hence is essential in $M$, which is again a contradiction to the hyperbolicity of $M$.

\section{The case $n_{a}=2, n_{b}=3$ or 4 , and $\Gamma_{1}, \Gamma_{2}$ non- positive}

Throughout this section we assume that $n_{a}=2, n_{b}=3$ or 4 , and both $\Gamma_{1}, \Gamma_{2}$ are non-positive. We will show that in this case there are only three possibilities for the pair $\left(\Gamma_{a}, \Gamma_{b}\right)$, given in Figures 16.6, 16.8 and 16.9. The following lemma rules out the possibility that $n_{b}=3$.

Lemma 16.1 The case $n_{a}=2, n_{b}=3$ and $\Gamma_{a}, \Gamma_{b}$ non-positive, is impossible.

Proof. The graph $\Gamma_{a}$ contains at most one loop at each vertex as otherwise it would contain a Scharlemann bigon, which contradicts Lemma 2.2(4) because $n_{b}=3$ implies that $\hat{F}_{b}$ is non-separating. There are at most four families of edges on $\Gamma_{a}$ connecting $u_{1}$ to $u_{2}$, containing a total of at least $\Delta n_{b}-2 \geq 10$ edges, hence there is a family containing 3 edges $e_{1} \cup e_{2} \cup e_{3}$. These are positive edges in $\Gamma_{b}$, and we may assume that $e_{i}$ has label $i$ at $u_{1}$. Since one of the vertices of $\Gamma_{b}$, say $v_{1}$, is anti-parallel to the other two vertices, the edge $e_{1}$ is a loop on $\Gamma_{b}$, so its label on $u_{2}$ is also 1 . Since $u_{1}, u_{2}$ are antiparallel, we see that the label of $e_{i}$ at $u_{2}$ is $i$ for $i=1,2,3$, hence they are all co-loop edges on $\Gamma_{a}$. This is a contradiction to the 3-Cycle Lemma 2.14(2).

We will assume in the remainder of this section that $n_{b}=4$. By Lemma 13.1 the graph $\hat{\Gamma}_{a}$ is as shown in Figure 13.1. Note that $\hat{e}_{1}, \hat{e}_{2}$ are on the same side of the loop at each $u_{i}$. Denote by $w_{i}$ the weight of $\hat{e}_{i}$, and put $\lambda=w_{5}=w_{6}$. Then we can denote $\Gamma_{a}$ by $\left(\lambda ; w_{1}, w_{2}, w_{3}, w_{4}\right)$, and by Lemma 13.1(2) we may 
assume that $w_{3}+w_{4} \leq w_{1}+w_{2}=6$ or 8. By Lemmas 2.3(1) and 2.3(3) we have $\lambda, w_{i} \leq 4$. Also, counting the number of edges incident to $u_{i}$ gives

$$
\sum_{i=1}^{4} w_{i}+2 \lambda=4 \Delta
$$

Lemma 16.2 (1) If $w_{i} \geq 3$ then $s_{i}=2$, where $s_{i}$ is the transition number of $\hat{e}_{i}$.

(2) $v_{1}$ is parallel to $v_{3}$ and antiparallel to $v_{2}$ and $v_{4}$.

(3) $\left(w_{1}, w_{2}\right)$ and $\left(w_{3}, w_{4}\right)$ cannot be $(3,2),(3,3)$ or $(3,4)$.

Proof. (1) Let $s_{i}$ be the transition number of $\hat{e}_{i}$. By the 3-Cycle Lemma $(2.14(2))$ we have $s_{i} \neq 0$. If $s_{1}= \pm 1$ then all vertices of $\Gamma_{b}$ would be parallel, which is a contradiction to the assumption that $\Gamma_{b}$ is non-positive. Since $n_{b}=4$, the only remaining possibility is that $s_{i}=2$.

(2) If $\lambda \geq 3$ then $\Gamma_{a}$ contains a Scharlemann cycle among the loops, so $\hat{F}_{b}$ is separating and the result follows. If $\lambda \leq 2$ then the equation $\sum w_{i}+2 \lambda=4 \Delta$ gives $w_{i} \geq 3$ for some $i$. By (1) and the parity rule, $v_{j}$ is parallel to $v_{j+2}$, hence the result follows because $\Gamma_{b}$ is non-positive.

(3) Assume $w_{1}=3$. By the equation above, $\lambda>0$, hence by Lemma 15.1 $w_{2}$ is odd. The transition function of $\hat{e}_{1}$ is given by (1), and it will determine that of $\hat{e}_{2}$. If $w_{2}=3$ then one can check that the transition function of $\hat{e}_{2}$ would map $j$ to $j$, which would be a contradiction to (1).

\section{Lemma $16.3 \lambda \geq 2$}

Proof. First assume $\lambda=0$. Then $\Gamma_{a}=(0 ; 4,4,4,4)$. By Lemma 16.2(1) all edges of $\Gamma_{a}$ have label pair $(1,3)$ or $(2,4)$, see Figure 16.1. Thus $\hat{\Gamma}_{b}$ is a union of two cycles, hence all edges from $v_{1}$ to $v_{3}$ in $\Gamma_{b}$ are equidistant. Since two of these edges are in $\hat{e}_{1}$ and are not equidistant on $\Gamma_{a}$, this is a contradiction to the Equidistance Lemma 2.17.

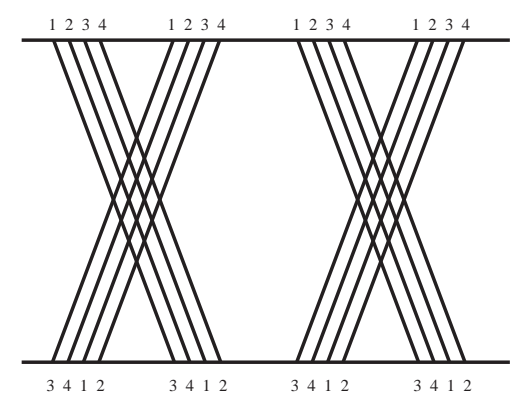

Figure 16.1

If $\lambda=1$, then $w_{j} \geq 2$ for $j=1, \ldots, 4$, so $w_{i} \neq 3$ by Lemma $16.2(3)$. Hence $\Gamma_{a}=(1 ; 4,4,4,2)$. One can check that the one of the families of weight 4 
would have the same label on the two endpoints of any of its edges, which is a contradiction to Lemma 16.2(1).

Lemma 16.4 Suppose $w_{i}=w_{j}=4$ and $\hat{e}_{i}=e_{1} \cup \ldots \cup e_{4}$ and $\hat{e}_{j}=e_{1}^{\prime} \cup \ldots \cup e_{4}^{\prime}$ satisfy (i) they have the same label sequence at $u_{1}$, and (ii) $e_{1}$ is equidistant to $e_{1}^{\prime}$ on $\Gamma_{a}$. Then there exist at least 4 non co-loop edges in the other two non-loop families of $\Gamma_{a}$.

Proof. The graph $\Gamma_{a}$ is as shown in Figure 16.2 for the case $(i, j)=(1,2)$. (The proof works in all cases.) Note that $e_{1}$ being equidistant to $e_{1}^{\prime}$ implies that $e_{k}$ is equidistant to $e_{k}^{\prime}$ for $k=1,2,3,4$. We may assume that the label sequence of $\hat{e}_{i}$ and $\hat{e}_{j}$ is $1,2,3,4$ at $u_{1}$. By Lemma 16.2(1) the four edges $e_{1} \cup \ldots \cup e_{4}$ form two essential cycles on $\Gamma_{b}$, so any edge on $\Gamma_{b}$ with endpoints $v_{1}, v_{3}$ must be parallel to $e_{1}$ or $e_{3}$. In particular, the edge $e_{1}^{\prime}$ has label pair $(1,3)$ and hence must be parallel to either $e_{1}$ or $e_{3}$. Note that two parallel positive edges are equidistant. Since $e_{1}^{\prime}$ is equidistant to $e_{1}$ and $e_{1}$ is not equidistant to $e_{3}$ on $\Gamma_{a}$, it follows that $e_{1}^{\prime}$ is not equidistant to $e_{3}$ on $\Gamma_{a}$, therefore by the Equidistance Lemma and the above we see that $e_{1}^{\prime}$ must be parallel to $e_{1}$ on $\Gamma_{b}$. Similarly each $e_{k}^{\prime}$ is parallel to $e_{k}$ on $\Gamma_{b}$. Since $e_{k}^{\prime}$ and $e_{k}$ have the same label $k$ at $u_{1}$ on $\Gamma_{a}$, they have the same label 1 at $v_{k}$ in $\Gamma_{b}$, so there must be another edge $e_{k}^{\prime \prime}$ between them. By the above $e_{k}^{\prime \prime}$ cannot be in $\hat{e}_{i} \cup \hat{e}_{j}$, hence they belong to the other two families of non-loop edges in $\Gamma_{a}$, and the result follows.

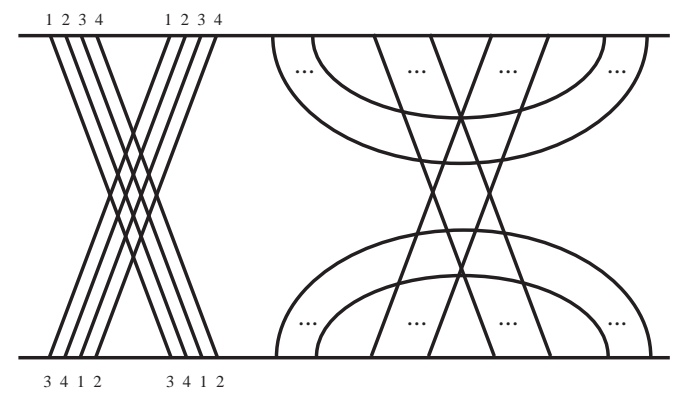

Figure 16.2

Lemma 16.5 $\lambda=3$ is impossible.

Proof. Suppose $\lambda=3$. Using the Congruence Lemma (Lemma 15.1) and Lemma 16.2(3) one can show that $\Gamma_{a}$ has the following possibilities.

(1) $\Delta=5,(3 ; 4,4,4,2)$;

(2) $\Delta=4,(3 ; 4,4,2,0)$;

(3) $\Delta=4,(3 ; 4,2,4,0)$;

(4) $\Delta=4,(3 ; 4,2,2,2)$. 
In each case, the family of $\hat{e}_{1}$ has weight 4 . We assume that its label sequence at $u_{1}$ is $1,2,3,4$. Then by Lemma 16.2(1) its label sequence at $u_{2}$ is $3,4,1,2$, which then completely determines the labels of $\Gamma_{a}$. One can check that in case (1) and (3) the family $\hat{e}_{3}$ gives 4 parallel co-loops, which is a contradiction to the 3-Cycle Lemma (Lemma 2.14(2)). Case (2) is impossible by Lemma 16.4.

It remains to consider case (4). The graph $\Gamma_{a}$ is shown in Figure 16.3. The third edge $A$ of $\hat{e}_{1}$ and the second edge $B$ of $\hat{e}_{3}$ in the figure both have label pair $(1,3)$. As in the proof of Lemma 16.4, this implies that they are parallel on $\Gamma_{b}$. Since $\hat{\Gamma}_{a}$ has at most 4 negative edges and at most 2 positive edges, by Lemma 2.2(2) $\Gamma_{b}$ cannot have more than $2 n_{a}=4$ parallel edges, so the endpoints of $A$ and $B$ at $v_{3}$ are adjacent among the four edge endpoints labeled 1 at $v_{3}$. Since $\Delta=4$, the jumping number is \pm 1 , so the endpoints of $A, B$ at $u_{1}$ in $\Gamma_{a}$ are also adjacent among the four edge endpoints labeled 3 at $u_{1}$. This is a contradiction because this is not the case in Figure 16.3.

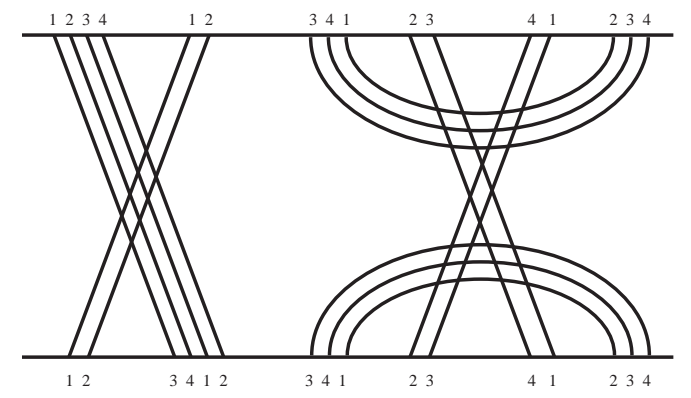

Figure 16.3

Lemma 16.6 If $\lambda=4$, then $\Gamma_{a}=(4 ; 4,2,4,2)$, and the graphs $\Gamma_{a}, \Gamma_{b}$ and their edge correspondence are as shown in Figure 16.6.

Proof. Since $\Gamma_{a}$ does not contain an extended Scharlemann cycle, by considering the labels at the endpoints of the four loops at $u_{1}$ we see that $w_{1}+w_{2} \equiv$ $w_{3}+w_{4} \equiv 2(\bmod 4)$. This, together with Lemmas 15.1 and $16.2(3)$, give the following possibilities for $\Gamma_{a}$.

(1) $\Delta=5, \Gamma_{a}=(4 ; 4,2,4,2)$;

(2) $\Delta=5, \Gamma_{a}=(4 ; 4,2,2,4)$;

(3) $\Delta=4, \Gamma_{a}=(4 ; 4,2,2,0)$.

We shall show that (2) and (3) are impossible, and (1) gives the example in Figure 16.6.

Case (2) can be excluded by Lemma 16.4. The graph $\Gamma_{a}$ is shown in Figure 16.4. Note that the corresponding edges of the two non-loop families of weight 4 are equidistant in $\Gamma_{a}$, and they have the same label sequence at $u_{1}$. Since the other two non-loop families of $\Gamma_{a}$ consist of co-loops, this is a contradiction to Lemma 16.4. 


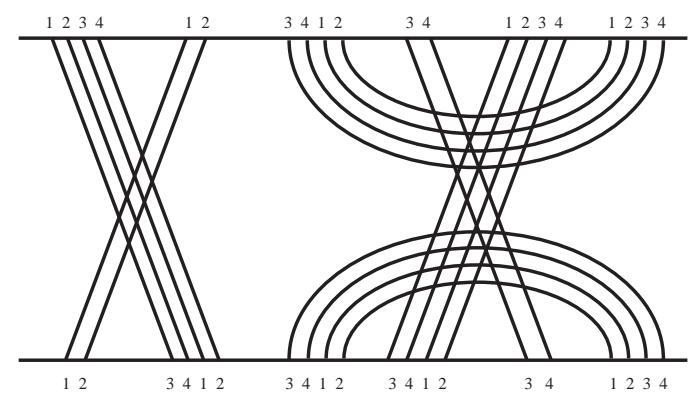

Figure 16.4

The graph for case (3) is shown in Figure 16.5. Note that there is a loop in $\Gamma_{b}$ based at each vertex $v_{i}$, so two edges connecting $v_{i}$ to different vertices must be on different sides of the loop. Consider the four edges with label 3 at $u_{1}$, indicated by $A, B, C, D$ in Figure 16.5. Note that they appear in this order on $\partial u_{1}$. Since $\Delta=4$, the jumping number is \pm 1 , so they must also appear in such an order on $\partial v_{3}$ in $\Gamma_{b}$.

On the other hand, since $A$ connects $v_{3}$ to $v_{1}$ while $B, D$ connect $v_{3}$ to $v_{4}, A$ must be on a different side of the loop $C$ at $v_{3}$ than $B, D$. Hence when traveling around $\partial v_{3}$ in a certain direction the four edges appear in the order $A, C, B, D$ or $A, C, D, B$. This is a contradiction. Therefore Case (3) is impossible.

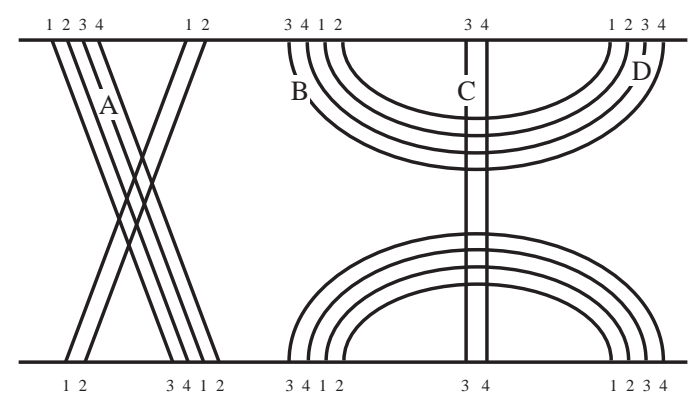

Figure 16.5

In case (1), the graph $\Gamma_{a}$ is shown in Figure 16.6(a). By the same argument as above, we see that the edges $B \cup E$ and $A \cup C$ must be on different sides of the loop $D$ in $\Gamma_{b}$. Therefore $B, E$ are adjacent among the 5 edges labeled 1 at $v_{3}$. Since they are not adjacent among the 3 -edges at $u_{1}$, the jumping number must be \pm 2 . This completely determines the edges around the vertex $v_{3}$ up to symmetry, which in turn determine the edges at adjacent vertices $v_{1}, v_{3}$ and then the edges at $v_{2}$. The graph $\Gamma_{b}$ is shown in Figure 16.6(b). 


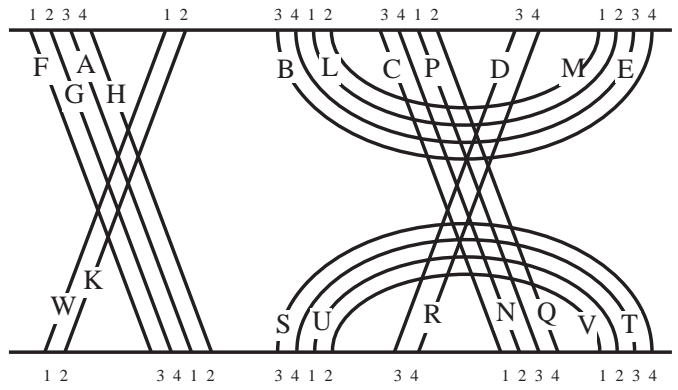

(a)

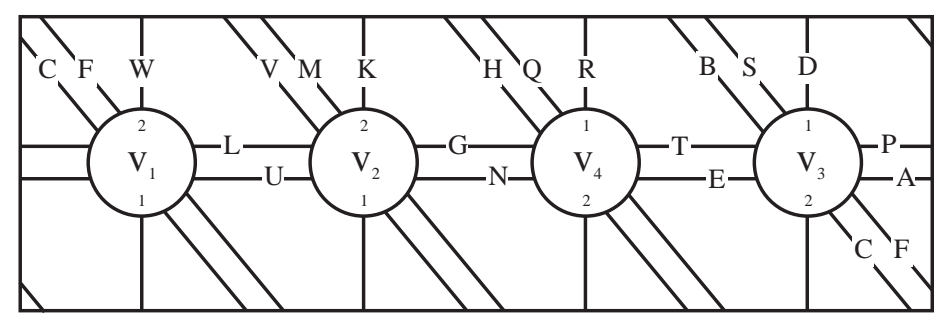

(b)

Figure 16.6

Lemma 16.7 If $\lambda=2$, then either $\Delta=5$ and $\Gamma_{a}=(2 ; 4,4,4,4)$, or $\Delta=4$ and $\Gamma_{a}=(2 ; 4,4,4,0)$. The graphs $\Gamma_{a}, \Gamma_{b}$ are as shown in Figures 16.8 and 16.9.

Proof. Here the possibilities for $\Gamma_{a}$ are

(1) $\Delta=5, \Gamma_{a}=(2 ; 4,4,4,4)$;

(2) $\Delta=4, \Gamma_{a}=(2 ; 4,4,4,0)$;

(3) $\Delta=4, \Gamma_{a}=(2 ; 4,4,2,2)$;

(4) $\Delta=4, \Gamma_{a}=(2 ; 4,2,4,2)$;

(5) $\Delta=4, \Gamma_{a}=(2 ; 4,2,2,4)$.

The graphs in cases (3) - (5) are shown in Figure 16.7 (a) - (c). In cases (3) and (4) the corresponding edges in the two weight 4 families are equidistant, and the other two non-loop families are co-loops. Therefore these cases are impossible by Lemma 16.4. In case (5) there are loops at $v_{1}$ and $v_{2}$ in $\Gamma_{b}$, and there is a (34)-Scharlemann bigon in $\Gamma_{a}$ which forms another essential cycle $C$ in $\Gamma_{b}$. Consider the two edges of $\Gamma_{a}$ with label 3 at $u_{1}$ and label 1 at $u_{2}$. On $\Gamma_{b}$ these edges connect $v_{3}$ and $v_{1}$, and therefore must lie on the same side of $C$. Hence they are adjacent among the four edges labeled 1 at $v_{3}$ because the other two edges connect $v_{3}$ to $v_{4}$. Since $\Delta=4$, the jumping number must be \pm 1 , so these edges are also adjacent among the four edges with label 3 at $u_{1}$, which is a contradiction because on Figure 16.7(c) the two edges with label 3 at $u_{1}$ and 
1 at $u_{2}$ are not adjacent among the four edges labeled 3 at $u_{1}$. Therefore (5) is also impossible.

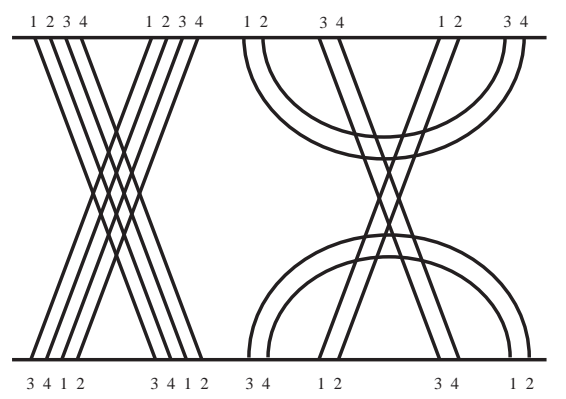

(a)

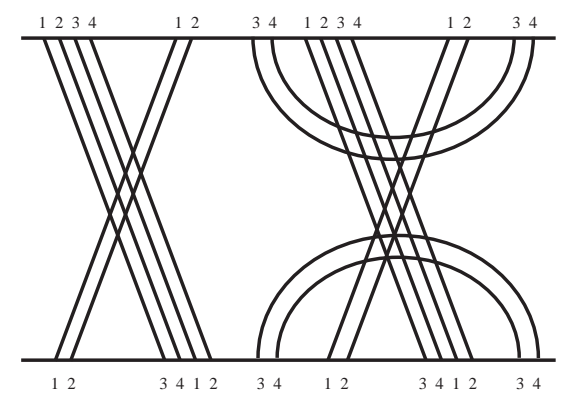

(b)

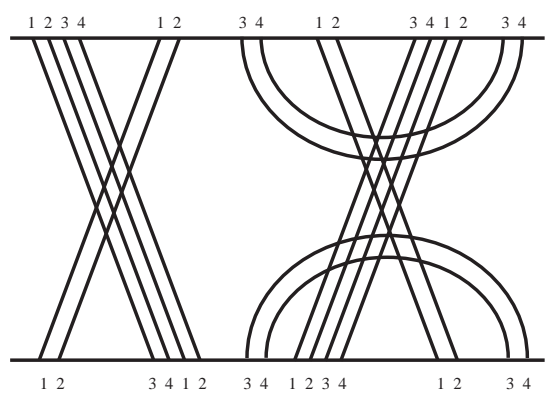

(c)

Figure 16.7

In case (1) the graph $\Gamma_{a}$ is shown in Figure 16.8(a). Label the edges as in the figure, and orient non-loop edges of $\Gamma_{a}$ from $u_{1}$ to $u_{2}$. As in the proof of Lemma 16.4, the $i$-th edge $e_{i}$ in $\hat{e}_{1}$ must be parallel to the $i$-th edge $e_{i}^{\prime}$ in $\hat{e}_{2}$ on $\Gamma_{b}$, and there is an edge of $\hat{e}_{3} \cup \hat{e}_{4}$ between them because $e_{i}, e_{i}^{\prime}$ both have label 1 at $v_{i}$. For the same reason the $i$-th edge of $\hat{e}_{3}$ is parallel to the $i$-th edge of $\hat{e}_{4}$, hence the positive edges of $\Gamma_{b}$ form four families of weight 4 . The two edges $e_{i}, e_{i}^{\prime}$ are adjacent among the five edges labeled 1 at $v_{i}$ in $\Gamma_{b}$, hence the jumping number $J= \pm 1$. Reversing the orientation of the vertices of $\Gamma_{b}$ if necessary we may assume $J=1$. We may also assume that the vertices $v_{1}, v_{3}$ are oriented counterclockwise and $v_{2}, v_{4}$ clockwise, otherwise we may look at $\hat{F}_{b}$ from the other side.

Since $\Gamma_{b}$ contains 4 parallel positive edges, by Lemma 13.2(2) $\Gamma_{a}$ is kleinian, so the weight of edges of $\hat{\Gamma}_{b}$ are all even. There are only two (14)-edges $K, W$ in $\Gamma_{a}$, so they must be parallel in $\Gamma_{b}$. They may appear in the order $(K, W)$ or $(W, K)$ on $\partial v_{1}$, but there is a homeomorphism of $\left(\hat{F}_{a}, \Gamma_{a}\right)$ which is label 
preserving, interchanging $u_{1}, u_{2}$ and mapping $K$ to $W$, hence up to symmetry we may assume that the order is $(K, W)$. Thus up to symmetry we may assume that $K$ and $W$ appear in $\Gamma_{b}$ as shown in Figure 16.8(b). This, together with the orientation of the vertices and the fact that $J=1$, completely determines the edges around $v_{1}$ and $v_{4}$, and then the edges around $v_{2}$ and $v_{3}$. See Figure $16.8(\mathrm{~b})$.

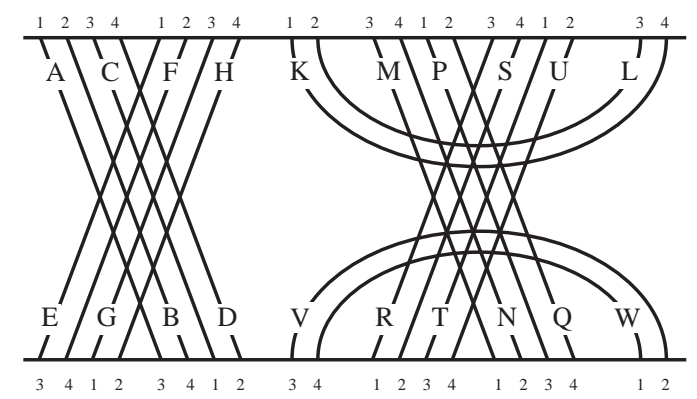

(a)

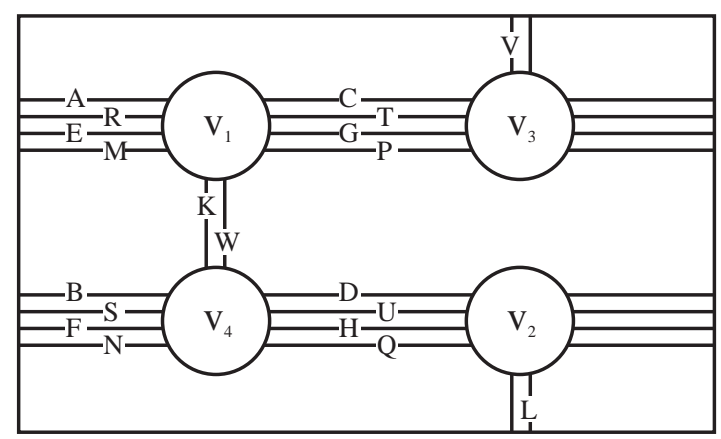

(b)

Figure 16.8

The graph $\Gamma_{a}$ in case (2) is shown in Figure 16.9(a). As above, one can show that each edge $e_{i}$ in $\hat{e}_{1}$ is parallel to $e_{i}^{\prime} \in \hat{e}_{2}$ and $e_{i}^{\prime \prime} \in \hat{e}_{3}$, where $e_{i}, e_{i}^{\prime}$ have label $i$ at $u_{1}$ and $e_{i}^{\prime \prime}$ has label $i$ at $u_{2}$. Orient $v_{i}$ as above. Up to symmetry we may assume $J=1$, and $A, E$ on $\Gamma_{b}$ are as shown in Figure 16.9(b). This determines $P$ and the position of $K$ at $\partial v_{1}$, and hence the labels of the 2-edges at $v_{1}$. The 4-labels at $u_{1}$ appear in the order $K, D, H, N$, so on $\Gamma_{b}$ they appear in this order around $v_{4}$, clockwise, hence $D, H$ must be to the right of $v_{4}$ in the figure. This also determines the 2-edges at $v_{4}$. In particular, the edges $K$ and $S$ must be non-parallel. The remaining two edges $R$ and $L$ can be determined similarly, using labels at $v_{2}$ and $v_{3}$. See Figure 16.9(b). 


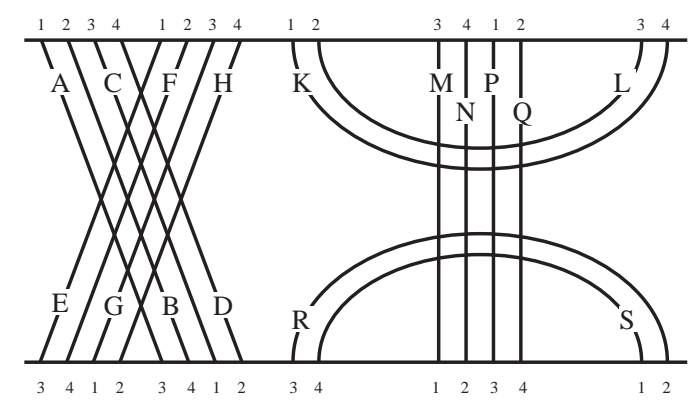

(a)

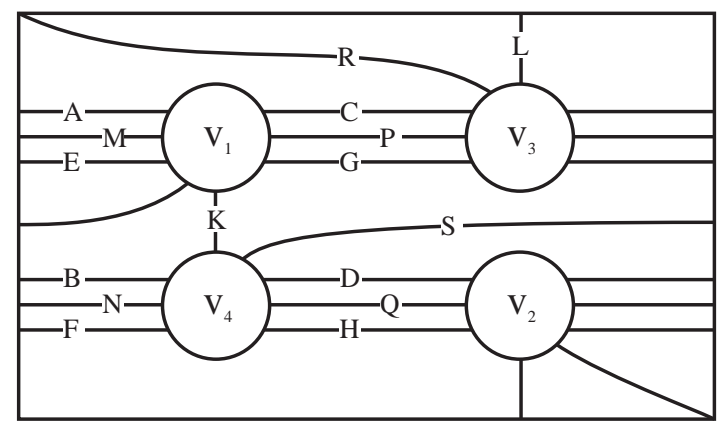

(b)

Figure 16.9

Proposition 16.8 Suppose $n_{a} \leq 2$ and $n_{b} \geq 3$. Then $\Gamma_{a}, \Gamma_{b}$ and their edge correspondence are given in Figure 11.9, 11.10, 14.5, 16.6, 16.8 or 16.9.

Proof. First assume that $\Gamma_{a}$ is positive. Then $n_{b} \leq 4$ by Lemma 3.2. By Lemma $2.23 n_{b}$ must be even, hence our assumption implies that $n_{b}=4$. By Proposition 11.10 the graphs are as shown in Figure 11.9 or 11.10.

Now assume $\Gamma_{a}$ is non-positive. Then we have $n_{a}=2$. The case that $\Gamma_{b}$ is positive has been ruled out by Proposition 12.17. Hence $\Gamma_{a}, \Gamma_{b}$ are both nonpositive. By Lemma $16.1 n_{b}$ cannot be 3. By Proposition 14.7 if $n_{b}>4$ then $\Gamma_{a}, \Gamma_{b}$ are given in Figure 14.5. Finally if $n_{b}=4$. Then Lemma 16.3 and 16.5 says that $\lambda=4$ or 2 , which are covered by Lemmas 16.6 and 16.7, respectively, showing that if $\lambda=4$ then the graphs are in Figure 16.6, and if $\lambda=2$ then the graphs are the pair in Figure 16.8 or 16.9. 


\section{Equidistance classes}

The next few sections deal with the case that $n_{i} \leq 2$ for $i=1,2$. In this section we introduce the concept of equidistance classes. The main properties are given in Lemmas 17.1 and 17.2, which will be used extensively in the next few sections.

Define a relation on the set of edges $E_{a}$ of $\Gamma_{a}$ such that $e_{1} \sim e_{2}$ if and only if (i) they have the same label pair, (ii) they have the same endpoint vertices, and (iii) they are equidistant.

Lemma 17.1 This is an equivalence relation.

Proof. We need only show that condition (iii) is transitive, i.e. if $e_{1}, e_{2}, e_{3}$ are edges on a graph $\Gamma$ such that $e_{1}, e_{2}$ and $e_{2}, e_{3}$ are equidistant pairs, then $e_{1}, e_{3}$ are equidistant.

By definition we have $d_{u_{1}}\left(e_{1}, e_{2}\right)=d_{u_{2}}\left(e_{2}, e_{1}\right)$, and $d_{u_{1}}\left(e_{2}, e_{3}\right)=d_{u_{2}}\left(e_{3}, e_{2}\right)$, hence $d_{u_{1}}\left(e_{1}, e_{3}\right)=d_{u_{1}}\left(e_{1}, e_{2}\right)+d_{u_{1}}\left(e_{2}, e_{3}\right)=d_{u_{2}}\left(e_{2}, e_{1}\right)+d_{u_{2}}\left(e_{3}, e_{2}\right)=d_{u_{2}}\left(e_{3}, e_{1}\right)$. This completes the proof.

We will call this equivalence relation the $E D$ relation. An equivalence class is then called an ED class, and the number of ED classes is called the $E D$ number of $\Gamma_{a}$, denoted by $\eta_{a}=\eta\left(\Gamma_{a}\right)$. We can then define $D_{a}=D\left(\Gamma_{a}\right)=$ $\left(c_{1}, \ldots, c_{\eta_{a}}\right)$, where $c_{i}$ are the number of edges of the equivalence classes, ordered lexicographically.

Lemma 17.2 Let $\Gamma_{a}, \Gamma_{b}$ be intersection graphs. Then the edge correspondence between the graphs induces a one to one correspondence between the ED classes of $\Gamma_{a}$ and $\Gamma_{b}$; in particular $\eta\left(\Gamma_{a}\right)=\eta\left(\Gamma_{b}\right)$, and $D\left(\Gamma_{a}\right)=D\left(\Gamma_{b}\right)$.

Proof. Note that $e_{1}, e_{2}$ satisfy (i) on $\Gamma_{a}$ if and only if they satisfy (ii) on $\Gamma_{b}$. The Equidistance Lemma 2.17 now says that a pair of edges are equivalent on $\Gamma_{a}$ if and only if they are equivalent on $\Gamma_{b}$.

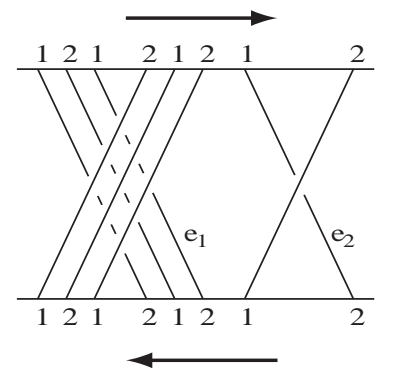

(a)

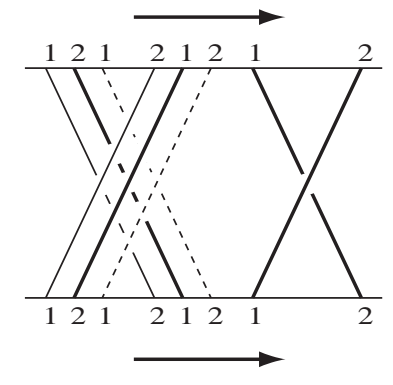

(b)

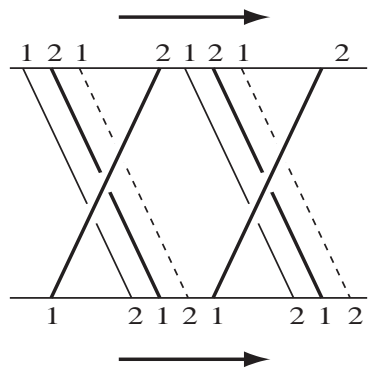

(c)

Figure 17.1

Example 17.3 (1) Consider a graph $\Gamma_{a}=+(3,3,1,1)$ and assume $n_{b}=2$, see Figure 17.1(a). In general if $n_{b}=2$ then all parallel positive edges are in the 
same ED class because they have the same label pairs and they are equidistant. One can check that non-parallel edges are not equidistant. (For example, let $u_{1}$ be the top vertex, $u_{2}$ the bottom vertex, and let $e_{1}, e_{2}$ be as shown in the figure; then $d_{u_{1}}\left(e_{1}, e_{2}\right)=4 \neq 2=d_{u_{2}}\left(e_{2}, e_{1}\right)$.) Hence $D\left(\Gamma_{a}\right)=(3,3,1,1)$. Compare this with $\hat{\Gamma}_{a}=+(3,1,3,1)$, in which case the two families of 3 edges are equidistant, and the other two families of weight 1 are equidistant, hence $D(+(3,1,3,1))=(6,2)$.

(2) Consider $\Gamma_{a}=-(3,3,1,1)$ or $-(3,1,3,1)$, and suppose that the edges of $\Gamma_{a}$ are not co-loops (hence conditions (i) and (ii) in the definition of ED equivalence are satisfied), see Figure 17.1(b) and (c). Equidistant edges are indicated in the figure by different kind of lines. We can see that $D(-(3,3,1,1))=$ $D(-(3,1,3,1)=(4,2,2)$.

(3) When $\Gamma_{a}=-(4,2,2,0)$, each of the middle edges of the family of 4 is equidistant to one edge in each of the two weight 2 families, and the other two edges of the weight 4 families are not equidistant to any other edges. Hence $D(-(4,2,2,0))=(3,3,1,1)$

(4) Suppose $\Gamma_{a}=+(4,2,2,0)$ and all edges have label pair (12). Then one can check that each family of parallel edges forms an ED class, hence $D\left(\Gamma_{a}\right)=$ $(4,2,2)$.

(5) Suppose $\Gamma_{a}=+(2,2,2,2)$ and all edges have label pair (12). Then one can show that the first family is equidistant to the third family, but not to the adjacent families. Hence $D\left(\Gamma_{a}\right)=(4,4)$.

(6) Similarly if $\Gamma_{a}=+(4,4,0,0)$ and all edges have the same label pair then $D\left(\Gamma_{a}\right)=(8)$.

\section{The case $n_{b}=1$ and $n_{a}=2$}

Lemma 18.1 Suppose $n_{a}=2$ and $n_{b}=1$. Then one of the following holds.

(1) $\Gamma_{a}=-(1,1,1,1)$ and $\Gamma_{b}=(4,0,0)$.

(2) $\Gamma_{a}=-(2,2,0,0)$ and $\Gamma_{b}=(2,2,0)$.

(3) $\Gamma_{a}=-(2,1,1,1)$ and $\Gamma_{b}=(3,1,1)$. The graphs $\Gamma_{a}, \Gamma_{b}$ and their edge correspondence are given in Figure 18.2.

Proof. In this case $\hat{\Gamma}_{b}$ has a single vertex, and $\hat{\Gamma}_{a}$ has two vertices of opposite orientation and has no loops. Hence we have $\Gamma_{a}=\left(a_{1}, \ldots, a_{4}\right)$, and $\Gamma_{b}=\left(b_{1}, b_{2}, b_{3}\right)$. We have $b_{1}+b_{2}+b_{3}=\Delta$. If $b_{i}, b_{j}$ are non-zero and $b_{i}+b_{j}$ is odd then one can check that one of the $\hat{e}_{i}, \hat{e}_{j}$ is a family of co-loops, which is a contradiction to the parity rule. Hence $b_{i} \equiv b_{j} \bmod 2$ for all $b_{i}, b_{j}$ non-zero. Thus if $\Delta=5$ then up to symmetry we have $\Gamma_{b}=(3,1,1)$, and if $\Delta=4$ then $\Gamma_{b}=(4,0,0)$ or $(2,2,0)$.

If $\Gamma_{b}=(4,0,0)$ then the four parallel edges are mutually non-parallel on $\Gamma_{a}$, hence $\Gamma_{a}=-(1,1,1,1)$.

If $\Gamma_{b}=(2,2,0)$, one can check that edges in different families are not equidistant, hence $D\left(\Gamma_{b}\right)=(2,2)$. Since each pair of parallel edges contributes one edge to each of two families in $\Gamma_{a}$, we have $\Gamma_{a}=-(2,2,0,0),-(2,1,1,0)$ or 
$-(1,1,1,1)$. When $\Gamma_{a}=-(2,1,1,0)$ the two single edges are equidistant, while each of the two parallel edges form an ED class, so $D\left(\Gamma_{a}\right)=(2,1,1) \neq D\left(\Gamma_{b}\right)$. Also, when $\Gamma_{a}=-(1,1,1,1)$ we have $D\left(\Gamma_{a}\right)=(4)$. Therefore in this case we have $\Gamma_{a}=-(2,2,0,0)$.

No suppose $\Gamma_{b}=(3,1,1)$. In this case the three parallel edges are equidistant, and each of the other two edges is not equidistant to any other edges. Hence $D\left(\Gamma_{b}\right)=(3,1,1)$. Since the three parallel edges in $\Gamma_{b}$ are mutually nonparallel on $\Gamma_{a}, \hat{\Gamma}_{a}$ has at least three edges. One can show that $D(-(2,2,1,0))=$ $(2,2,1) \neq D\left(\Gamma_{b}\right)$, hence $\hat{\Gamma}_{a} \neq-(2,2,1,0)$. Therefore $\Gamma_{a}=-(3,1,1,0)$ or $-(2,1,1,1)$.

In the case that $\Gamma_{a}=-(3,1,1,0)$ and $\Gamma_{b}=(3,1,1)$, the graphs are as shown in Figure 18.1. The three parallel edges $B, C, E$ are equidistant, hence they represent the two weight 1 edges $\hat{e}_{2}, \hat{e}_{3}$ and the middle edge of the weight 3 edge $\hat{e}_{1}$, so the other two edges $A, D$ must be as shown in Figure 18.1(a) up to symmetry. Since they are non-adjacent at $u_{1}$ and their label 1 endpoints are non-adjacent among the five label 1 edge endpoints at $v_{1}$ in $\Gamma_{b}$, the jumping number must be \pm 1 . This determines the edge correspondence between $\Gamma_{a}$ and $\Gamma_{b}$, as shown in Figure 18.1.

The torus $\hat{F}_{a}$ cuts $M\left(r_{a}\right)$ into two components. Let $W$ be the one containing the bigon $\alpha$ on $F_{b}$ bounded by $B \cup E$ and the 3 -gon $\beta$ bounded by $A \cup C \cup D$. It can be constructed by attaching a 1-handle representing part of the Dehn filling solid torus, then two 2 -handles represented by $\alpha, \beta$, then a 3 -cell. The fundamental group of $W$ is generated by the horizontal circle $x$ and the vertical circle $y$ shown in the figure, and the 1-handle $z$ from $u_{2}$ to $u_{1}$. On the boundary of $\alpha, \beta$, $A, B, C, D, E$ represent $1, x, x y, 1,1$, respectively, and each corner represents $z$, hence $\alpha, \beta$ give the relations $z z x=1$ and $z x y z z=1$, respectively. Solving these in $x$ and $y$ shows that $\pi_{1}(W)=\mathbb{Z}$, generated by $z$. It follows that $\hat{F}_{a}$ is not $\pi_{1}$-injective in $W$, and hence is compressible. This is a contradiction.
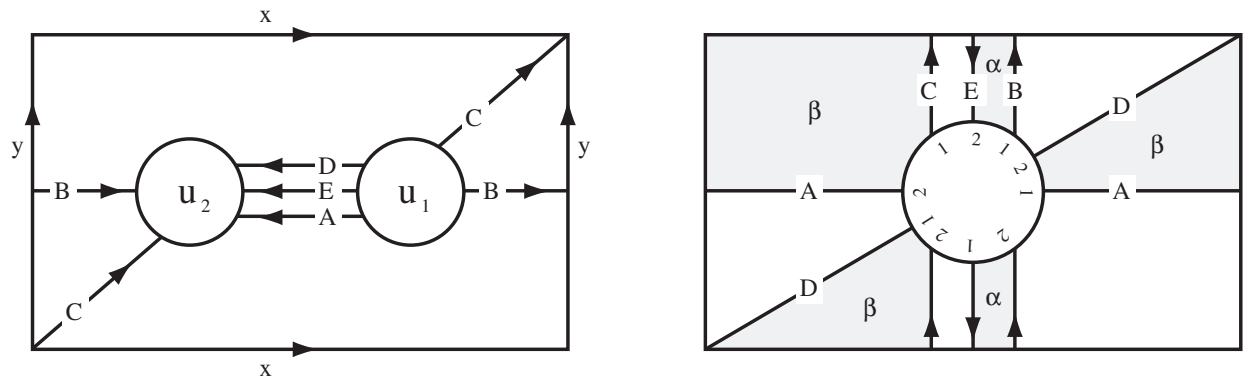

Figure 18.1

We now have $\Gamma_{a}=-(2,1,1,1)$ and $\Gamma_{b}=(3,1,1)$. The three parallel edges $B, C, E$ are equidistant, hence on $\Gamma_{a}$ they are the single edges because they are equidistant to each other but not to the edges in the weight 2 family. Since the 
edge endpoints of these are consecutive on $\partial v_{1}$ while the 1-label endpoint of $E$ at $v_{1}$ is not adjacent to that of either $B$ or $C$, the jumping number must be \pm 2 . This determines the correspondence of the edges up to symmetry, see Figure 18.2 .
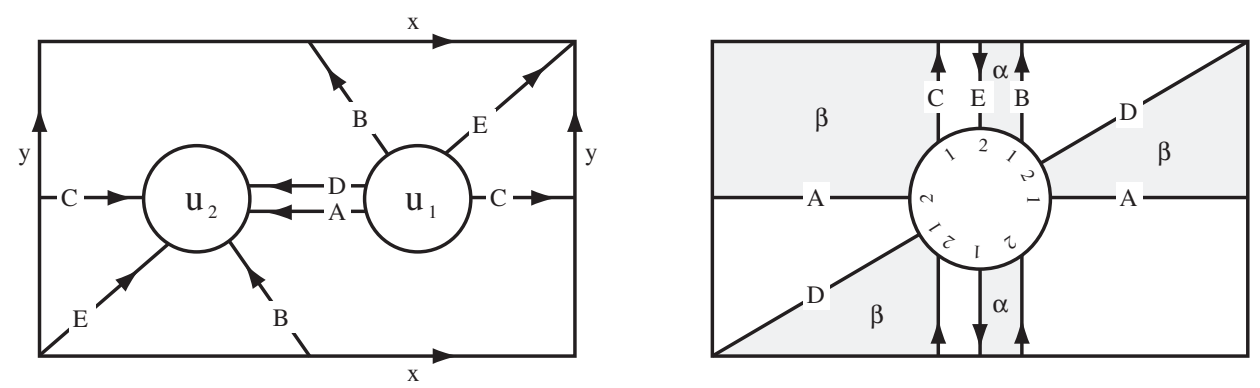

Figure 18.2

\section{The case $n_{1}=n_{2}=2$ and $\Gamma_{b}$ positive}

In this section we assume that $n_{1}=n_{2}=2$ and $\Gamma_{b}$ is positive. Then no edge of $\hat{\Gamma}_{a}$ is a loop, hence $\Gamma_{a}=-\left(a_{1}, \ldots a_{4}\right)$, and $\Gamma_{b}=+\left(\rho ; b_{1}, \ldots, b_{4}\right)$.

When $\rho \neq 0$ we may rearrange the $a_{i}$ to write $\Gamma_{a}=-\left(r_{1}, \ldots, r_{p} \mid s_{1}, \ldots, s_{q}\right)$, where $r_{i}$ are the weights of the co-loop edges, and $s_{j}$ are the weights of the non co-loop edges.

Lemma 19.1 Suppose $n_{1}=n_{2}=2$ and $\Gamma_{b}$ is positive.

(1) All non-zero $b_{i}$ are of the same parity, all non-zero $r_{j}$ are of the same parity, all non-zero $s_{k}$ are of the same parity, and the non-zero $r_{j}$ and $s_{k}$ are of opposite parity.

(2) $r_{i} \leq 2, s_{j} \leq 4$, and $\rho+b_{k} \leq 4$.

(3) $2 \rho+\sum b_{i}=2 \Delta$, and $\sum r_{i}+\sum s_{j}=2 \Delta$.

Proof. (1) This follows from the Congruence Lemma 15.1.

(2) Since $\hat{\Gamma}_{b}$ has at most two loops, each co-loop family of $\Gamma_{a}$ contains at most two edges, hence $r_{i} \leq 2$. Similarly, since $\hat{\Gamma}_{b}$ has at most four non-loop edges, $s_{j} \leq 4$. On $\Gamma_{b}, \rho$ is the number of edges in a loop family, which is no more than $p$, the number of co-loop edges in $\hat{\Gamma}_{a}$. Similarly, $b_{k}$ is no more than $q$, the number of non co-loop edges in $\hat{\Gamma}_{a}$. Since $\hat{\Gamma}_{a}$ has at most 4 edges, we have $\rho+b_{k} \leq 4$.

(3) This follows from the fact that each vertex of $\Gamma_{a}$ or $\Gamma_{b}$ has valence $2 \Delta$. 
Lemma 19.2 Suppose $n_{1}=n_{2}=2$ and $\Gamma_{b}$ is positive. If $\rho=4$ then $\Gamma_{a}=$ $-(2,2,2,2)$, and $\Gamma_{b}=+(4 ; 0,0,0,0)$.

Proof. Since $\rho+b_{j} \leq 4$ (Lemma 19.1(2)), we have $b_{i}=0$ for all $i$, hence from Lemma 19.1(3) we have $\Delta=4$. Thus $\hat{\Gamma}_{b}$ is a union of two disjoint loops, each representing a family of four edges. Since each family of four parallel edges in $\hat{\Gamma}_{b}$ contributes one edge to each family in $\Gamma_{a}$, we have $\Gamma_{a}=-(2,2,2,2)$.

Lemma 19.3 Suppose $n_{1}=n_{2}=2$ and $\Gamma_{b}$ is positive. Then $\rho \neq 3$.

Proof. Suppose $\rho=3$. The three loops in a family represent different classes on $\hat{\Gamma}_{a}$, so $\hat{\Gamma}_{a}$ has at least three co-loop edges. Since $\Gamma_{b}$ has some non-loop edges, $\hat{\Gamma}_{a}$ has at least one non co-loop edge. It follows that $\hat{\Gamma}_{a}$ has exactly three co-loop edges, so $\Gamma_{a}=-\left(2,2,2 \mid s_{1}\right)$. Since $\sum r_{i}+\sum s_{j}=2 \Delta$ is even, $s_{1}$ is even, which contradicts Lemma 19.1(1).

Lemma 19.4 Suppose $n_{1}=n_{2}=2$ and $\Gamma_{b}$ is positive. Then $\rho \neq 2$.

Proof. On $\hat{\Gamma}_{a}$ there are non-co-loop edges, so there are at most three co-loop edges, but since $r_{i} \leq 2$ and $\sum r_{i}=4$ and the $r_{i}$ 's are of the same parity, there must be exactly two co-loop edges. Hence $\Gamma_{a}=-\left(2,2 \mid s_{1}, s_{2}\right)$. By the Congruence Lemma, the $s_{i}$ are odd, hence either $\Delta=5$ and $\Gamma_{a}=-(2,2 \mid 3,3)$, or $\Delta=4$ and $\Gamma_{a}=-(2,2 \mid 3,1)$.

If $\Delta=4$ and $\Gamma_{a}=-(2,2 \mid 3,1)$, then from Lemma 19.1 we have $b_{i} \leq$ $2, \sum b_{i}=4$, and $b_{i} \equiv b_{j} \bmod 2$ if $b_{i}, b_{j} \neq 0$. These conditions give $\Gamma_{b}=$ $+(2 ; 1,1,1,1),+(2 ; 2,2,0,0)$ or $+(2 ; 2,0,2,0)$. One can check that in the first two cases the four non-loop edges of $\Gamma_{b}$ form two equidistance classes of 2 edges each, so $D_{b}=(2,2,2,2)$, and in the third case the four non-loop edges are all equidistant to each other, so $D_{b}=(4,2,2)$. On the other hand, the three parallel edges of $\Gamma_{a}$ belong to distinct classes, and there are at least two co-loop classes, hence $\eta\left(\Gamma_{a}\right) \geq 5$. This is a contradiction to Lemma 17.2.

If $\Delta=5$ and $\Gamma_{a}=-(2,2 \mid 3,3)$, then from Lemma 19.1 we have $b_{i} \leq 4-\rho=$ $2, \sum b_{i}=6$, and $b_{i} \equiv b_{j} \bmod 2$ if $b_{i}, b_{j} \neq 0$, so we must have $\Gamma_{b}=+(2 ; 2,2,2,0)$. Depending on the weight sequence of the edges of $\hat{\Gamma}_{a}$, we have $\Gamma_{a}=-(3,2,3,2)$ or $-(3,3,2,2)$. If $\Gamma_{a}=-(3,2,3,2)$ then from the labeling one can see that the two edges with both endpoints labeled 1 are not equidistant. Since these are parallel loops at $v_{1}$ of $\Gamma_{b}$, they are equidistant on $\Gamma_{b}$, which is a contradiction to the Equidistance Lemma 2.17. Therefore $\Gamma_{a} \neq(-3,2,3,2)$.

Now suppose $\Gamma_{a}=-(3,3,2,2)$, and $\Gamma_{b}=+(2 ; 2,2,2,0)$. Then the graphs are as shown in Figure 19.1. Consider the edges $A, B, C, D, E$ with label 1 at $u_{1}$ of $\Gamma_{a}$. These correspond to the 5 edges with label 1 at $v_{1}$ of $\Gamma_{b}$. Note that on $\Gamma_{a} D, E$ are co-loop edges, hence on $\Gamma_{b}$ they are the two loops at $u_{1}$. Since their endpoints with label 1 are not adjacent among the 1-edges at $v_{1}$ in $\Gamma_{b}$, the jumping number must be \pm 2 , so among these edges in $\Gamma_{b}$, the edge $B$ is the one in $\Gamma_{b}$ which is adjacent to both $D$ and $E$ at $v_{1}$, as shown in the Figure. Now consider the five edges labeled 2 at $u_{2}$. Note that they appear in the order 
$C A B F G$. Using the same argument as above we see that the edge $A$ is the one adjacent to both $F$ and $G$, so we would have $A=B$, which is a contradiction.

$\mathrm{u}_{1}$

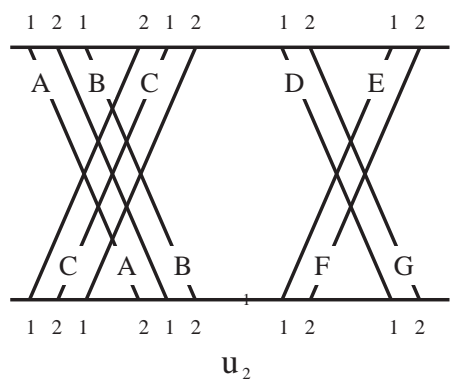

$\mathrm{v}_{1}$

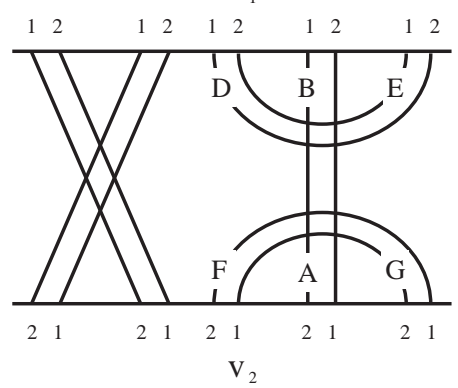

Figure 19.1

Lemma 19.5 Suppose $n_{1}=n_{2}=2$ and $\Gamma_{b}$ is positive. Then $\rho \neq 1$.

Proof. Suppose $\rho=1$. Then $\Gamma_{a}$ has two co-loop edges, hence $\Gamma_{a}=-\left(1,1 \mid s_{1}, s_{2}\right)$ or $-\left(2 \mid s_{1}, s_{2}, s_{3}\right)$.

If $\Delta=5$ then the second case does not happen since by the Congruence Lemma $s_{i}$ would be 0 or odd and $\sum s_{i}=8$, which would give $s_{i}>4$ for some $i$, contradicting Lemma 19.1(2). Therefore $\Gamma_{a}=-(1,1 \mid 4,4)$. Since each weight 4 family contributes one edge to each non-loop family of $\Gamma_{b}$, we have $\Gamma_{b}=+(1 ; 2,2,2,2)$. Now the graph $\hat{\Gamma}_{b}$ contains both black and white bigons, whose edges all belong to the two weight 4 families on $\Gamma_{a}$. This is a contradiction to Lemma 15.2.

If $\Delta=4$ then $\Gamma_{a}=-(1,1 \mid 4,2)$ or $-(2 \mid 3,3,0)$. The first case cannot happen because the $\hat{e}_{i}$ of weight 4 contributes one edge to each family in $\hat{\Gamma}_{b}$, while the edge of weight 2 contributes one edge to each of two families, so $\Gamma_{b}=+(1 ; 2,2,1,1)$, which contradicts the Congruence Lemma. In the second case for the same reason above we must have $\Gamma_{b}=+(1 ; 2,2,2,0)$. Again there are black and white bigons, which contradicts Lemma 15.2 because on $\Gamma_{a}$ the edges of these bigons all belong to the two weight 3 families.

Lemma 19.6 Suppose $n_{1}=n_{2}=2$ and $\Gamma_{b}$ is positive. If $\rho=0$ then $\Delta=5$.

Proof. In this case there is no loop on either graph, hence by Lemma 19.1(1) all non-zero $a_{i}$ have the same parity, and all non-zero $b_{j}$ have the same parity. Any two edges connect the same pair of vertices and have the same pair of labels on their two endpoints, hence by definition they are ED equivalent if and only if they are equidistant. 
Assume $\Delta=4$. By the Congruence Lemma each of $\hat{\Gamma}_{a}$ and $\Gamma_{b}$ is of type $(4,4,0,0),(2,2,2,2),(4,2,2,0),(3,1,3,1)$, or $(3,3,1,1)$. Let $e_{1} \cup e_{2}$ be a bigon on $\Gamma_{b}$. Then $e_{1}$ and $e_{2}$ are equidistant on $\Gamma_{b}$, so by Lemma 2.17 they form an equidistant pair on $\Gamma_{a}$. Note that since $e_{1}$ and $e_{2}$ are not loops on $\Gamma_{b}$, on $\Gamma_{a}$ they have different labels on $u_{1}$. On the other hand, one can check that if $\Gamma_{a}=-(4,4,0,0)$ or $-(2,2,2,2)$ then an equidistant pair $e_{1}, e_{2}$ on $\Gamma_{a}$ must have the same label on $u_{1}$, which is a contradiction. Therefore $\Gamma_{a}=-(4,2,2,0)$, $-(3,3,1,1)$ or $-(3,1,3,1)$. (Note that the above argument does not apply to $\hat{\Gamma}_{b}$ since a pair of parallel edges on $\Gamma_{a}$ is not an equidistant pair.) We will rule these out one by one.

CLAIM 1. The case $\Gamma_{a}=-(4,2,2,0)$ is impossible.

If $\Gamma_{a}=-(4,2,2,0)$ then $b_{i} \neq 0$ for all $i$, hence $\Gamma_{b}=+(2,2,2,2)$ or $+(3,1,3,1)$, or $+(3,3,1,1)$. In the first case all black (say) faces of $\Gamma_{b}$ are bigons, so Lemma 13.2(2) implies that $\Gamma_{b}$ is kleinian because $\hat{\Gamma}_{a}$ has more than two edges. Since $\hat{\Gamma}_{a}$ has a single edge of weight 4 , it will be fixed by the free involution given in Lemma 6.2(4), which is absurd. By Example 17.3 we have $D(-(4,2,2,0))=(3,3,1,1)$, and $D(+(3,1,3,1))=(6,2)$, hence $\Gamma_{b} \neq+(3,1,3,1)$. It follows that $\Gamma_{b}=+(3,3,1,1)$. The graphs are shown in Figure 19.2. Each of $A, D$ on $\Gamma_{a}$ forms an equidistance class, hence they are the single edges on $\Gamma_{b}$. Up to symmetry we may assume that $A, D$ are as shown in Figure 19.2(b). This and the jumping number $J$ determines the edge correspondence of the graphs. The case that $J=1$ is shown in the figure. When $J=-1$ the edges $G, E$ would be equidistant on $\Gamma_{a}$ but not on $\Gamma_{b}$, which is impossible.
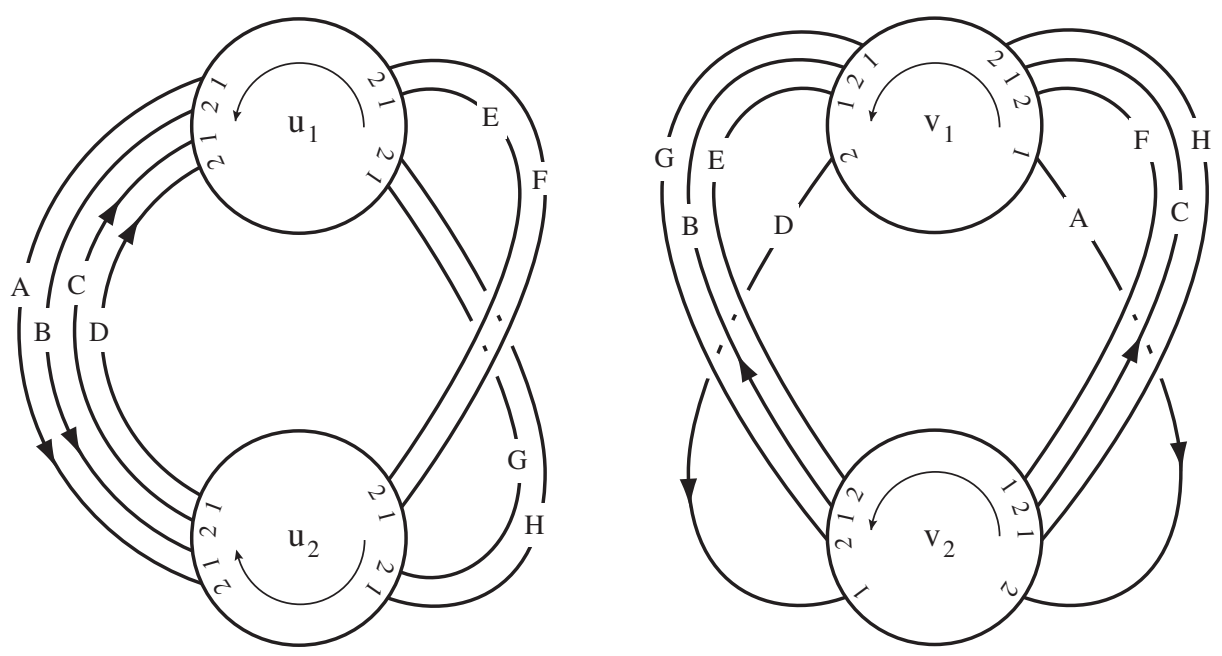

Figure 19.2

Let $P_{1}, P_{2}$ be the bigon disks on $F_{a}$ bounded by $A \cup B$ and $C \cup D$, respectively. Then the union of $P_{1}, P_{2}$ and two disks on $T_{0}$ form an annulus $Q$. More explicitly, 
let $a_{1}$ (resp. $b_{1}$ ) be the arc on $\partial u_{1}$ (resp. $\partial u_{2}$ ) from the endpoint of $A$ to that of $B, a_{2}$ (resp. $b_{2}$ ) the arc on $\partial u_{1}$ (resp. $\partial u_{2}$ ) from the endpoint of $C$ to that of $D, a_{3}$ (resp. $\left.b_{3}\right)$ on $\partial v_{1}$ (resp. $\partial v_{2}$ ) from $A$ to $C$, and $a_{4}$ (resp. $b_{4}$ ) on $\partial v_{2}$ (resp. $\partial v_{1}$ ) from $B$ to $D$. Then $a_{1} \cup \ldots \cup a_{4}$ (resp. $b_{1} \cup \ldots \cup b_{4}$ ) bounds a disk $P_{3}$ (resp. $P_{4}$ ) on the boundary torus $T_{0}$. Now $Q=P_{1} \cup \ldots \cup P_{4}$ is an annulus in $M$. Note that $\partial Q$ consists of two simple closed curves $\partial_{1}=A \cup C \cup a_{3} \cup b_{3}$ and $\partial_{2}=B \cup D \cup a_{4} \cup b_{4}$.

Orient $A, B$ to point from $u_{1}$ to $u_{2}$ on $\Gamma_{a}$. This determines the orientation of $\partial_{i}$. Note that they are parallel on the annulus $Q$. On $\Gamma_{b}$ the orientations of $A, B$ are from label 1 to label 2 , as shown in the figure. This determines the orientation of $C, D$. It is important to see that $\partial_{1}, \partial_{2}$ are parallel as oriented curves on $\hat{F}_{b}$. Let $Q^{\prime}$ be an annulus on $\hat{F}_{b}$ with $\partial Q^{\prime}=\partial_{1} \cup \partial_{2}$. Then $Q \cup Q^{\prime}$ is a non-separating torus (not a Klein bottle!) in $M\left(r_{b}\right)$ intersecting the Dehn filling solid torus at a single meridian disk, which contradicts the choice of $\hat{F}_{b}$. Therefore this case is impossible.

CLAIM 2. The case $\Gamma_{a}=-(3,1,3,1)$ is impossible. If $\Gamma_{a}=-(3,3,1,1)$ then $\Gamma_{b}=+(4,2,2,0)$.

Now suppose $\Gamma_{a}=-(3,3,1,1)$ or $-(3,1,3,1)$ and $\Gamma_{b}=+(4,4,0,0),+(4,2,2,0)$, $+(2,2,2,2),+(3,3,1,1)$ or $+(3,1,3,1)$. By Example 17.3 we have $D(-(3,3,1,1))=$ $-(3,1,3,1)=(4,2,2)$. On the other hand, by Example 17.3 we also have $D(+(4,4,0,0))=(8), D(+(4,2,2,0))=(4,2,2), D(+(2,2,2,2))=(4,4), D(+(3,3,1,1))=$ $(3,3,1,1)$, and $D(+(3,1,3,1))=(6,2)$. Therefore by Lemma 17.2 in this case we must have $\hat{\Gamma}_{b}=+(4,2,2,0)$. If $\hat{\Gamma}_{a}=-(3,1,3,1)$ then the four edges in the same ED class all have label 2 (say) at $u_{1}$, which means that on $\Gamma_{b}$ they all have label 1 at $v_{2}$, so they cannot be the four parallel edges in $+(4,2,2,0)$. Therefore $\hat{\Gamma}_{a} \neq-(3,1,3,1)$.

CLAIM 3. The case $\hat{\Gamma}_{a}=-(3,3,1,1)$ is impossible.

By Claim 2 we have $\hat{\Gamma}_{b}=+(4,2,2,0)$. The graphs are as shown in Figure 19.3. While the graphs are similar to that in Figure 19.2, the argument is necessarily different because the orientation of the vertices of $\Gamma_{b}$ here are parallel while that of $\Gamma_{a}$ in Figure 19.2 are antiparallel. One can check that up to symmetry the edge correspondence must be as shown in the figure.

We would like to apply Lemma 2.15 to get a contradiction. To do that, let $Q$ be the face of $\Gamma_{a}$ bounded by $A \cup B \cup E \cup H$. The edge $B$ is parallel to $C$ on $\Gamma_{b}$, and $C$ is a non-border edge on $\Gamma_{a}$, hence one of the bigons $C \cup H$ or $C \cup F$ is a coupling face $Q^{\prime}$ of $Q$ along the edge $B$. By Lemma 2.15 there is a rel $\partial$ isotopy of $F_{a}$ such that the new intersection graph $\Gamma_{a}^{\prime}$ is obtained from $\Gamma_{a}$ by deleting $A$ and $E$ and adding two edges parallel to $B$ and $F$, respectively. It follows that $\Gamma_{a}^{\prime}=-(4,2,2,0)$. This is impossible by Claim 1 . Therefore the case $\hat{\Gamma}_{a}=-(3,3,1,1)$ is also impossible. 

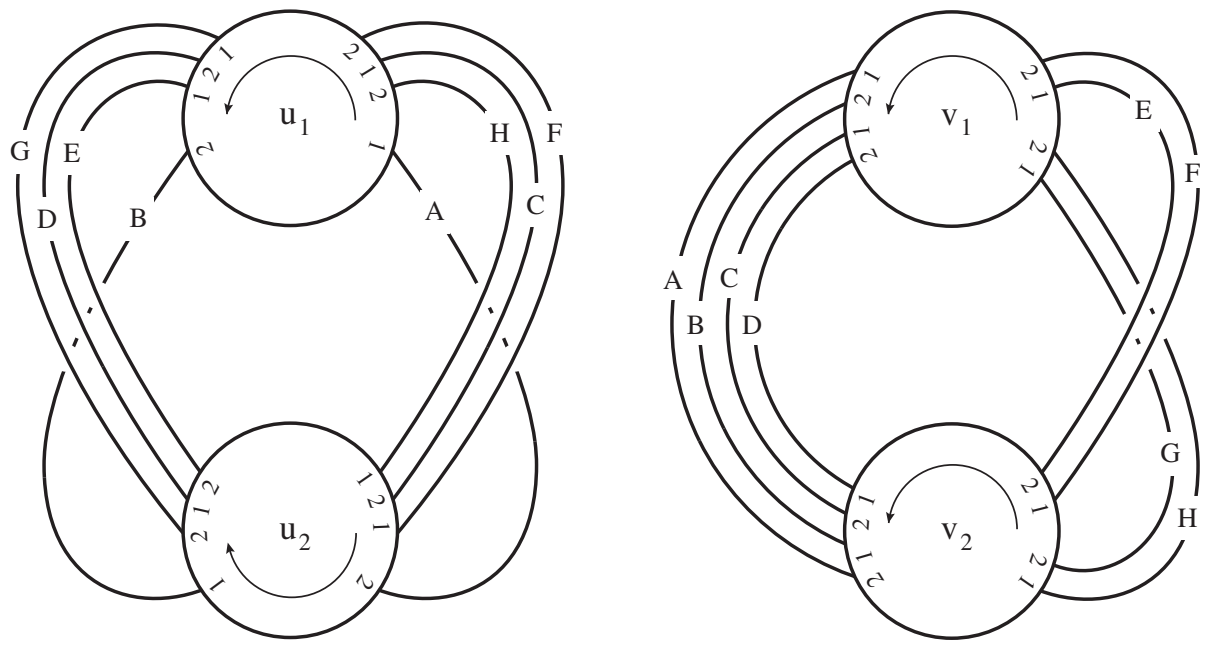

Figure 19.3

Lemma 19.7 Suppose $n_{1}=n_{2}=2$ and $\Gamma_{b}$ is positive. If $\rho=0$ then $\Gamma_{a}=$ $-(3,3,3,1)$ and $\Gamma_{b}=+(3,3,3,1)$. The graphs $\Gamma_{a}, \Gamma_{b}$ and their edge correspondence are shown in Figure 19.4.

Proof. By Lemma 15.1 all non-zero $a_{i}$ have the same parity, and all nonzero $b_{j}$ have the same parity. By Lemma 19.6 we have $\Delta=5$, so each of $\Gamma_{a}$ and $\Gamma_{b}$ is of type $(4,4,2,0),(4,2,2,2)$ or $(3,3,3,1)$. If some $b_{i}=4$ then by Lemma 13.2(2) $\Gamma_{a}$ is kleinian, but since each of the above type has an edge whose weight is non-zero and different from the others, it must be mapped to itself by the involution in Lemma 6.2(4), which is a contradiction because it is supposed to be a free involution on $\hat{F}_{a}$. It follows that $\Gamma_{b}=+(3,3,3,1)$. Direct calculation gives $D(+(3,3,3,1))=(4,3,3), D(-(4,4,2,0))=(3,3,2,2)$, $D(-(4,2,2,2))=(4,4,1,1)$, and $D(-(3,3,3,1))=(4,3,3)$. Hence by Lemma 17.2 we have $\Gamma_{a}=-(3,3,3,1)$. 

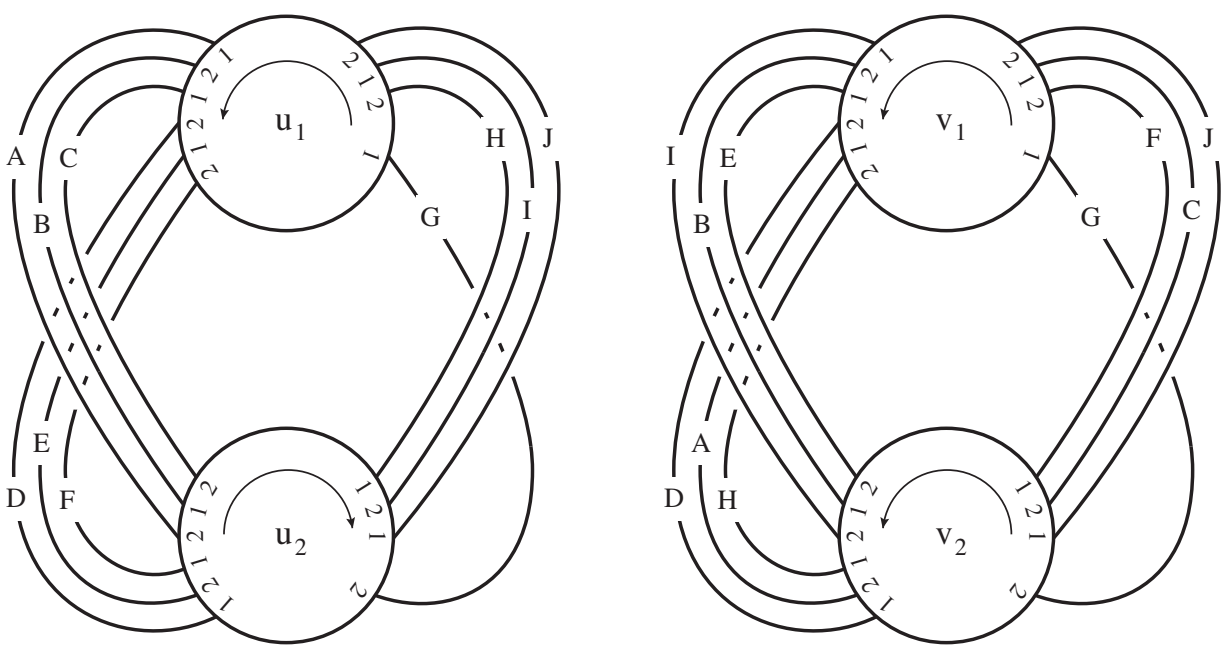

Figure 19.4

The graphs $\Gamma_{a}, \Gamma_{b}$ are as shown in Figure 19.4. Label the edges of $\Gamma_{a}$ as in the figure. Relabeling the vertices of $\Gamma_{b}$ if necessary, we may assume that the labels of edges of $\Gamma_{a}$ at $u_{1}$ are as shown. Since $\Gamma_{b}$ has no loops, each edge of $\Gamma_{a}$ has different labels on its two endpoints, which determines the labels at $u_{2}$. One can check that $\Gamma_{a}$ has three equidistance classes $c_{1}=\{B, E, G, I\}$, $c_{2}=\{A, D, H\}$ and $c_{3}=\{C, F, J\}$. Since $\Gamma_{b}$ is positive, each family belongs to an equidistance class; moreover, one can check that the single edge is equidistant to the non-adjacent family of weight 3 , which we will denote $\hat{e}_{1}$. Therefore these must belong to $c_{1}$. On $\Gamma_{a}, B$ has label 1 at $u_{2}$, so on $\Gamma_{b} B$ has label 2 at $v_{1}$. It follows that $B$ is the middle edge in $\hat{e}_{1}$. This determines the labels on $v_{1}$ and $v_{2}$. Now the endpoints of $E, G, I \in c_{1}$ are adjacent on $\partial u_{1}$ among edge endpoints labeled 1 , but they are not all adjacent on $\partial v_{1}$ because the single edge is not adjacent to those in $\hat{e}_{1}$ among edges with label 1 at $v_{1}$ in $\Gamma_{b}$. Therefore the jumping number $J$ cannot be \pm 1 , so $J= \pm 2$. Reversing the orientation of $v_{1}, v_{2}$ if necessary we may assume that $J=2$. Thus the edges $E, G, I$ in $\Gamma_{b}$ must be as shown. The other edges are now determined by this information. For example, the edges with label 2 at $u_{1}$ appear in the order $B, D, F, H, J$, so on $\Gamma_{b}$ the edges with label 1 at $v_{2}$ appear in the order $B, H, D, J, F$.

\section{The case $n_{1}=n_{2}=2$ and both $\Gamma_{1}, \Gamma_{2}$ non- positive}

In this section we assume that $n_{1}=n_{2}=2$ and both $\Gamma_{1}, \Gamma_{2}$ are non-positive. Let $\Gamma_{a}=\left(\rho_{a} ; a_{1}, \ldots, a_{4}\right)$, and $\Gamma_{b}=\left(\rho_{b} ; b_{1}, \ldots, b_{4}\right)$. Without loss of generality we may assume that $\rho_{b} \geq \rho_{a}$. 
Lemma 20.1 Suppose $n_{1}=n_{2}=2$, and $\Gamma_{1}, \Gamma_{2}$ are non-positive.

(1) $\Delta / 2 \leq \rho_{b} \leq 4$.

(2) $2 \rho_{a}+\sum a_{i}=2 \Delta$, and $2 \rho_{b}+\sum b_{i}=2 \Delta$.

(3) $a_{i}, b_{i} \leq 2$.

Proof. (1) Since a loop in $\Gamma_{a}$ corresponds to a non-loop in $\Gamma_{b}$ and vice versa, we have $\rho_{a}+\rho_{b}=\Delta$. We have assumed $\rho_{b} \geq \rho_{a}$, so $\rho_{b} \geq \Delta / 2$. Since no two edges are parallel on both graphs and $\hat{\Gamma}_{a}$ has at most four non-loop edges, we also have $\rho_{b} \leq 4$.

(2) This follows from the fact that the valence of a vertex in $\Gamma_{a}$ or $\Gamma_{b}$ is $2 \Delta$.

(3) Since $\Gamma_{a}$ and $\Gamma_{b}$ are non-positive, a non-loop edge in $\Gamma_{a}$ is a loop in $\Gamma_{b}$, hence there are at most two edges in each non-loop family of $\Gamma_{a}$, i.e. $a_{i} \leq 2$. Similarly for $b_{i}$.

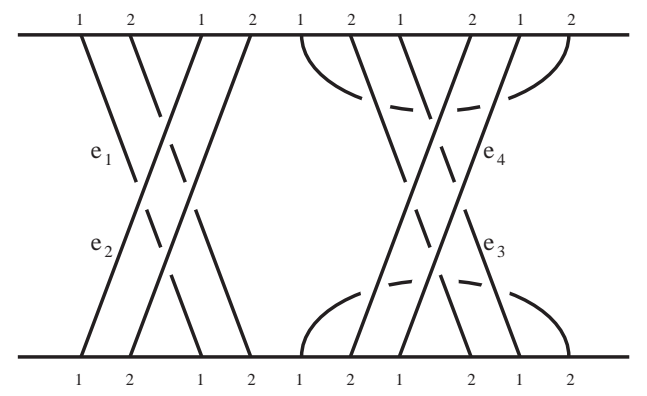

Figure 20.1

Lemma 20.2 Suppose $n_{1}=n_{2}=2, \Delta=5$, and $\Gamma_{1}, \Gamma_{2}$ are non-positive. Then $\Gamma_{a}=-(2 ; 2,2,2,0)$ and $\Gamma_{b}=-(3 ; 1,1,1,1)$ or $-(3 ; 2,2,0,0)$.

Proof. By Lemma 20.1(1) we have $\rho_{b}=3$ or 4 . If $\rho_{b}=4$ then each loop family contributes one edge to each non-loop family of $\Gamma_{a}$, hence $\Gamma_{a}=-(1 ; 2,2,2,2)$. The four loops $e_{1}, e_{2}, e_{3}, e_{4}$ at $v_{1}$ are equidistant to each other; on the other hand, from Figure 20.1 one can see that $e_{i}$ is equidistant to $e_{j}$ on $\Gamma_{a}$ if and only if $e_{i}$ and $e_{j}$ are on the same side of the loop at $u_{1}$. This is a contradiction. Therefore this case cannot happen.

Now assume $\rho_{b}=3$. Then by the Congruence Lemma we have $\Gamma_{b}=$ $-(3 ; 2,0,2,0),-(3 ; 2,2,0,0)$ or $-(3 ; 1,1,1,1)$. Since $\rho_{a}=\Delta-\rho_{b}=2$, we have $\sum a_{i}=10-2 \rho_{a}=6$. By Lemma 20.1 we have $a_{i} \leq 2$, therefore by the Congruence Lemma we must have $\Gamma_{a}=-(2 ; 2,2,2,0)$. The first case for $\Gamma_{b}$ above cannot happen because the two non-loop 1-edges are not equidistant in $\Gamma_{b}$ while as parallel loops on $\Gamma_{a}$ they are equidistant on $\Gamma_{a}$. Therefore $\Gamma_{b}=-(3 ; 2,2,0,0)$ or $-(3 ; 1,1,1,1)$. 


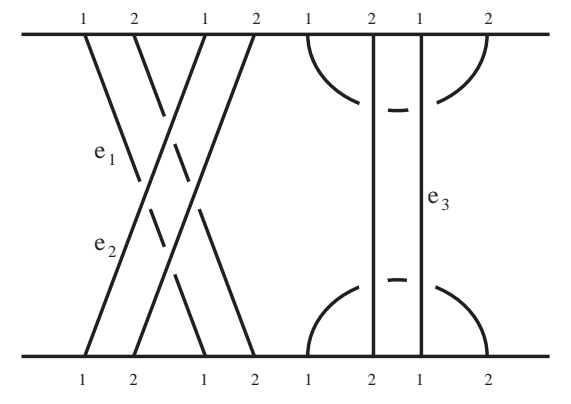

Figure 20.2

Lemma 20.3 Suppose $n_{1}=n_{2}=2, \Delta=4$, and $\Gamma_{1}, \Gamma_{2}$ are non-positive. Then one of the following holds.

(1) $\Gamma_{a}=-(2,2,2,2)$ and $\Gamma_{b}=-(4 ; 0,0,0,0)$.

(2) Both $\Gamma_{1}$ and $\Gamma_{2}$ are of type $-(2 ; 1,1,1,1)$.

(3) Both $\Gamma_{1}$ and $\Gamma_{2}$ are of type $-(2 ; 2,0,2,0)$.

(4) Both $\Gamma_{1}$ and $\Gamma_{2}$ are of type $-(2 ; 2,2,0,0)$.

Proof. If $\rho_{b}=4$ then $\Gamma_{b}=-(4 ; 0,0,0,0)$ and each loop family contributes one edge to each family of $\Gamma_{a}$, hence $\Gamma_{a}=-(2,2,2,2)$.

If $\rho_{b}=3$, then by the Congruence Lemma 15.1 we have $\Gamma_{a}=-(1 ; 2,2,2,0)$. Let $e_{1}, e_{2}, e_{3}$ be the three loops at $v_{1}$. As parallel positive edges, they are equidistant on $\Gamma_{b}$. On $\Gamma_{a}$ they are as shown in Figure 20.2. One can check that $e_{1}$ is equidistant to $e_{2}$ but not $e_{3}$, which is a contradiction to Lemma 2.17. Therefore $\rho_{b} \neq 3$.

When $\rho_{b}=\rho_{a}=2$, by the Congruence Lemma each of $\Gamma_{a}$ and $\Gamma_{b}$ is of type $-(2 ; 1,1,1,1)$ or $-(2 ; 2,2,0,0)$ or $-(2 ; 2,0,2,0)$. We are done if both $\Gamma_{a}, \Gamma_{b}$ are of the same type.

If $\Gamma_{a}=-(2 ; 2,2,0,0)$ then the two non-loop edges with label 1 at both endpoints are adjacent among the four edges labeled 1 at $u_{1}$, hence on $\Gamma_{b}$ the two loops at $u_{1}$ are adjacent among the four edges with label 1 at $v_{1}$, which implies that $\Gamma_{b}$ cannot be $-(2 ; 2,0,2,0)$ or $-(2 ; 1,1,1,1)$.

It remains to rule out the possibility that $\Gamma_{a}=-(2 ; 1,1,1,1)$ and $\Gamma_{b}=$ $-(2 ; 2,0,2,0)$. In this case the graphs are as shown in Figure 20.3.

Label the edges of $\Gamma_{a}$ as in the figure. We want to show that this determines the labels of the edges of $\Gamma_{b}$ up to symmetry. Since $\Delta=4$, by changing the orientation of $\hat{F}_{b}$ if necessary we may assume that the jumping number is 1 . The 1-edges at $u_{1}$ are in the order $A, B, C, D$, so these labels appear in this order at $v_{1}$ on $\Gamma_{b}$. The order of the 1-edges at $u_{2}$ is $A, X, C, Y$, so the 2-edges at $v_{1}$ are also in this order, which determines the edges $X, Y$ on $\Gamma_{b}$. Finally, the order of the 2-edges at $u_{1}$ determines the edges $E, F$ in $\Gamma_{b}$. Hence the labels of the graphs are as shown in Figure 20.3.

One way to see that these graphs are not realizable is to consider the annulus $A$ from $\partial v_{1}$ to $\partial v_{2}$ along the positive orientation, draw the segments of $\partial u_{1}, \partial u_{2}$ 
on this annulus and check that these arcs must intersect on $A$, which contradicts the fact that $\partial u_{1}, \partial u_{2}$ are parallel curves on the torus $T_{0}$. Here is another way. Consider the endpoints of the edges $D, X$, labeled $a, b, c, d$ on the two graphs. We have

$$
d_{v_{1}}(a, c)=d_{v_{2}}(b, d)=1
$$

so by Lemma 2.16 (applied with $u_{i}, u_{j}, v_{k}, v_{l}$ replaced by $v_{1}, v_{2}, u_{1}, u_{2}$ and $P, Q, R, S$ replaced by $a, c, b, d)$, we should have

$$
d_{u_{1}}(a, b)=d_{u_{2}}(c, d)
$$

However, on $\Gamma_{a}$ we have $d_{u_{1}}(a, b)=5$ while $d_{u_{2}}(c, d)=3$, which is a contradiction.
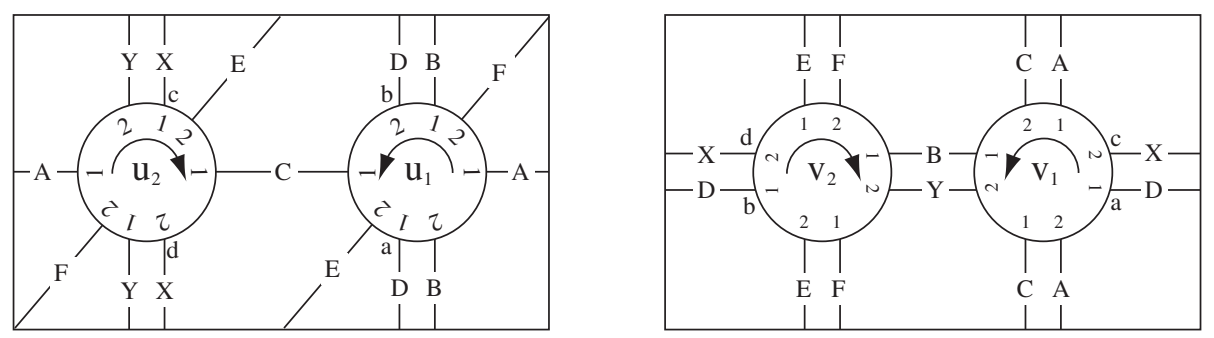

Figure 20.3

Proposition 20.4 Suppose $n_{a}, n_{b} \leq 2$. Then up to symmetry $\Gamma_{a}$ and $\Gamma_{b}$ are one of the following pairs.

$\begin{array}{lll}(1) & -(1,1,1,1) & (4,0,0) \\ (2) & -(2,2,0,0) & (2,2,0) \\ (3) & -(2,1,1,1) & (3,1,1) \\ (4) & -(2,2,2,2) & +(4 ; 0,0,0,0) \\ (5) & -(3,3,3,1) & +(3,3,3,1) \\ (6) & -(2 ; 2,2,2,0) & -(3 ; 1,1,1,1) \\ (7) & -(2 ; 2,2,2,0) & -(3 ; 2,2,0,0) \\ (8) & -(2,2,2,2) & -(4 ; 0,0,0,0) \\ (9) & -(2 ; 1,1,1,1) & -(2 ; 1,1,1,1) \\ (10) & -(2 ; 2,0,2,0) & -(2 ; 2,0,2,0) \\ (11) & -(2 ; 2,2,0,0) & -(2 ; 2,2,0,0)\end{array}$

Proof. This follows from the lemmas in Sections 18-20. More precisely, the case $n_{b}=1$ is done in Lemma 18.1, which gives (1)-(2) above; the case $n_{a}=n_{b}=2$ and $\Gamma_{b}$ positive is discussed in Lemmas 19.2-19.7 according to different numbers of loops on $\Gamma_{b}$, which gives $(3)-(5)$; the case $n_{a}=n_{b}=2$ with both graphs non-positive is discussed in Lemmas 20.2-20.3, with the possibilities listed in $(6)-(11)$. 
Proposition 20.5 For each of the cases (3), (5), (6), (9) and (10) of Proposition 20.4, the correspondence between edges of $\Gamma_{a}, \Gamma_{b}$ is unique up to symmetry, and is shown in Figures 18.2, 19.4, 20.4, 20.5 and 20.6, respectively.

Proof. For cases (3) and (5) this follows from Lemmas 18.1 and 19.7.

In case (6) we have $\Gamma_{a}=-(2 ; 2,2,2,0)$ and $\Gamma_{b}=-(3 ; 1,1,1,1)$. The graphs $\Gamma_{a}, \Gamma_{b}$ are as shown in Figure 20.4. Label the edges of $\Gamma_{b}$ as shown in the figure. By symmetry we may assume that the labels on the edge endpoints of $\Gamma_{b}$ are as in the figure. Also up to symmetry of $\Gamma_{b}$ on the torus $\hat{F}_{b}$ we may assume that the labels on $v_{1}$ are as in the figure.

The label 1 endpoints of $A, B, C$ are non-adjacent among the 1-labels on $\partial v_{1}$. These are non-loops on $\Gamma_{a}$, and one in each family, hence their endpoints at $u_{1}$ are also non-adjacent among endpoints labeled 1 . This forces the jumping number $J$ to be \pm 1 . Now on $\Gamma_{a}$ the edge $A$ must be as shown. It is easy to see that this determines the labels on the other edges in $\Gamma_{a}$.
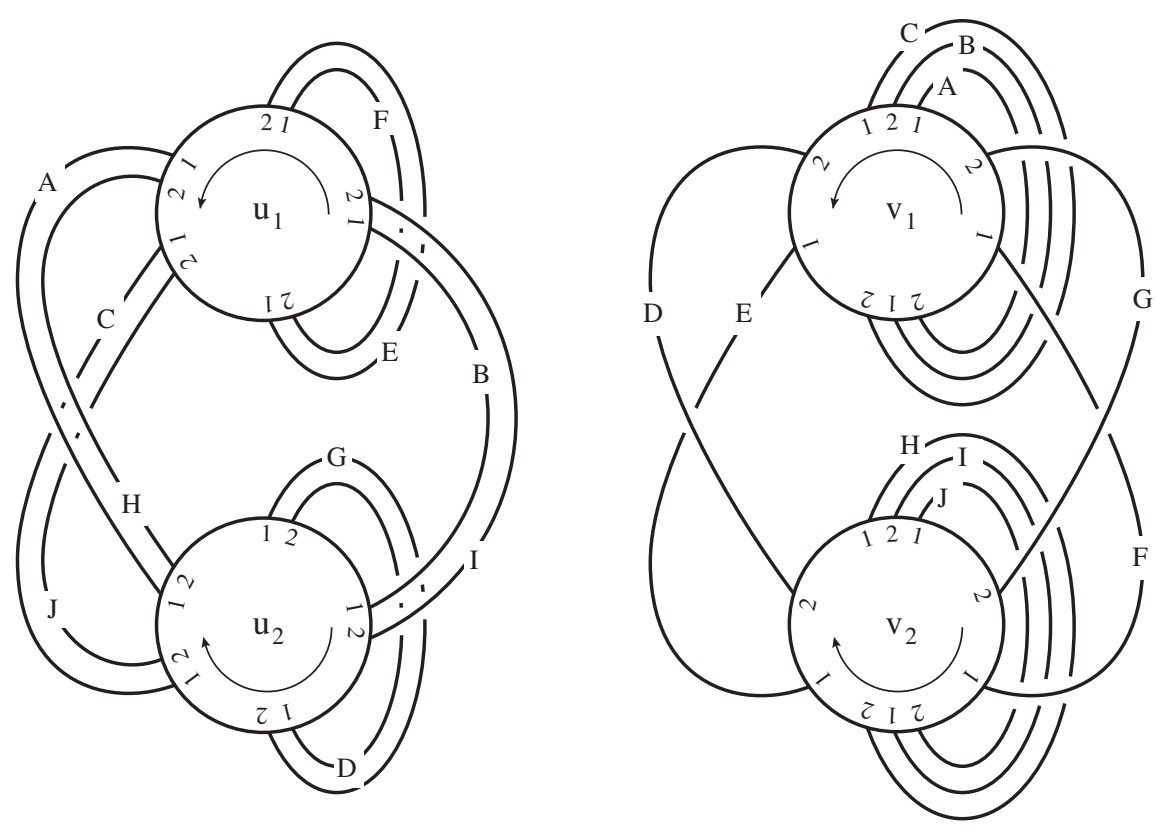

Figure 20.4

In case (9) we have $\Gamma_{a}=-(2 ; 1,1,1,1), \Gamma_{b}=-(2 ; 1,1,1,1)$, and $\Delta=4$, so we may assume $J=1$. Label edge endpoints and edges of $\Gamma_{a}$ as in the figure. Using symmetry we may assume $A$ to be any one of the two non-loop edges labeled 1 at $v_{1}$. Then this determines the labels on the other edges. See Figure 20.5 .

The determination of the edge correspondence for case (10) is similar. The graphs are shown in Figure 20.6. 


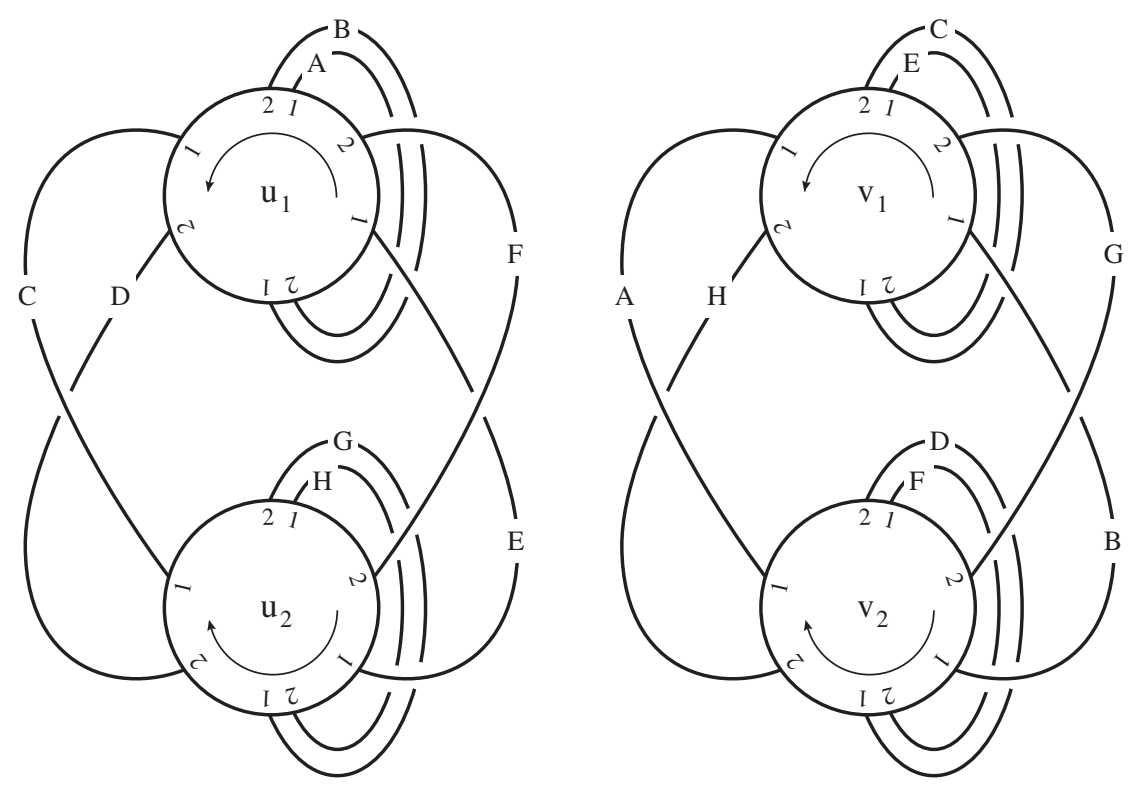

Figure 20.5
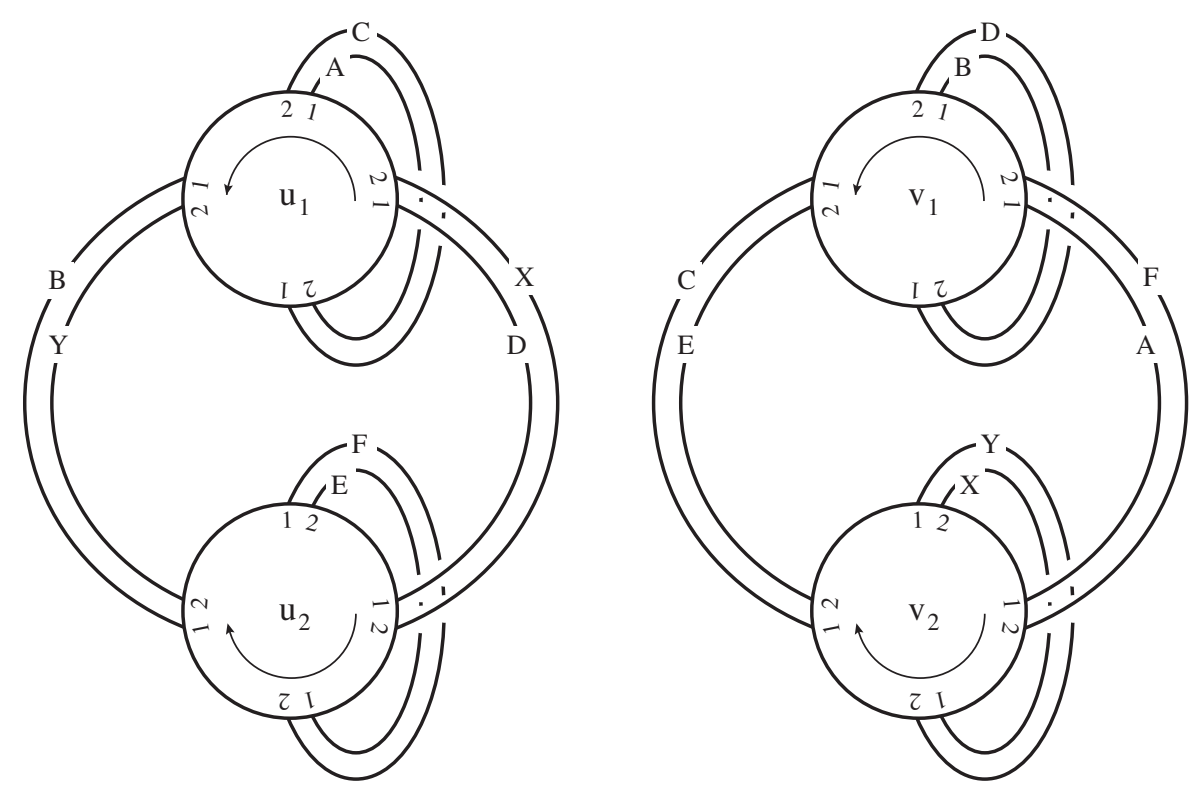

Figure 20.6 


\section{The main theorems}

Suppose $M$ is a hyperbolic manifold admitting two toroidal Dehn fillings $M\left(r_{1}\right), M\left(r_{2}\right)$. Let $F_{a}$ be essential punctured tori in $M$ such that $\partial F_{a}$ consists of a minimal number of copies of $r_{a}$, and $F_{1}$ intersects $F_{2}$ minimally. Let $X\left(F_{1}, F_{2}\right)$ be obtained from $N\left(F_{1} \cup F_{2} \cup T_{0}\right)$ by capping off its 2-sphere boundary components with 3-balls. We will use $X\left(r_{1}, r_{2}\right)$ to denote any $X\left(F_{1}, F_{2}\right)$ above with $\partial F_{a}$ of slope $r_{a}$, and call it a core of $M$ with respect to the toroidal slopes $r_{1}, r_{2}$. Note that $X\left(r_{1}, r_{2}\right)$ may not be unique.

Lemma 21.1 Suppose $M$ is a hyperbolic manifold admitting two toroidal Dehn fillings $M\left(r_{1}\right), M\left(r_{2}\right)$ of distance 4 or 5 . Then each of $\partial X\left(r_{1}, r_{2}\right)$ and $\partial M$ is a union of tori.

Proof. By the result of the previous sections we see that $\Gamma_{a}, \Gamma_{b}$ are either the graphs in Figures 11.9, 11.10, 14.5, 16.6, 16.8, 16.9, or one of the pairs given in Proposition 20.4.

In all figures except 11.9 and $11.10, \Gamma_{a}$ has two vertices and they have opposite signs. Now $X\left(F_{1}, F_{2}\right)$ can be constructed by adding thickened faces of $\Gamma_{b}$ to $N\left(F_{a} \cup T_{0}\right)$, which has two boundary components of genus 2 . It is easy to check that in all cases $\Gamma_{b}$ has at least one disk face on each side of $F_{a}$. The boundary of a disk face of $\Gamma_{b}$ is always an essential curve on $F_{a} \cup T_{0}$. Adding a 2handle corresponding to a disk face will change a genus 2 boundary component to one or two tori. It follows that the boundary of $X\left(F_{1}, F_{2}\right)$ is a union of tori. Since $M$ is irreducible and atoroidal, each torus boundary component of $X\left(F_{1}, F_{2}\right)$ either is boundary parallel, or bounds a solid torus. Therefore $\partial M$ is also a union of tori.

The proof for Figures 11.9 and 11.10 is similar. In these cases $\Gamma_{a}$ has 4 vertices, so $N\left(F_{a} \cup T_{0}\right)$ has two boundary components $S_{i}$ of genus 3 . It suffices to find two faces on each side of $F_{a}$ whose boundary curves give rise to nonparallel and non-separating curves on $S_{i}$. For 11.9 one can check that the bigons on $F_{b}$ bounded by the edges $K \cup J$ and $H \cup G$ are on the same side of $F_{a}$ and give non-parallel boundary curves on $S_{1}$, say, while the bigon bounded by the edges $J \cup H$ and the 3 -gon bounded by the edges $K \cup L \cup R$ give non-parallel non-separating curves on $S_{2}$. Hence the result follows. For 11.10, use the bigons bounded by $E \cup F$ and $G \cup H$ on one side, and the bigon $F \cup G$ and the 3-gon $E \cup K \cup A$ on the other side.

Consider the three manifolds $M_{1}, M_{2}, M_{3}$ in [GW1, Theorem 1.1]. More explicitly, $M_{1}$ is the exterior of the Whitehead link, $M_{2}$ is the exterior of the 2-bridge link associated to the rational number 3/10, and $M_{3}$ is the exterior of the $(-2,3,8)$ pretzel link, also known as the Whitehead sister link. Each of these manifolds admits two Dehn fillings $M_{i}\left(r_{1}\right)$ and $M_{i}\left(r_{2}\right)$, both toroidal and annular, with $\Delta=4$ for $i=1,2$, and $\Delta=5$ for $i=3$. Let $T_{0}$ be the Dehn filling component of $\partial M_{i}$, and let $T_{1}$ be the other component of $\partial M_{i}$. Then for all except a few slopes $s$ on $T_{1}, M^{\prime}=M_{i}(s)$ is a hyperbolic manifold, and it admits two toroidal Dehn fillings $M^{\prime}\left(r_{1}\right), M^{\prime}\left(r_{2}\right)$ of distance 4 or 5 . The 
following lemma shows that several of the cases in Proposition 20.4 can only be realized by these manifolds.

Lemma 21.2 Suppose $\Gamma_{a}$ has a non-disk face. Then $M=M_{i}$ or $M_{i}(s)$ for some $i=1,2,3$ and slope $s$ on $T_{1}$, and the toroidal slopes $r_{1}, r_{2}$ are the same as the toroidal/annular slopes given in [GW1, Theorem 1.1].

Proof. Let $K$ be a curve on $F_{a}$ which is essential on $\hat{F}_{a}$ and disjoint from $F_{a} \cap F_{b}$. Consider the manifold $X=M-\operatorname{Int} N(K)$. If $X$ is hyperbolic then $\hat{A}=\hat{F}_{a}-\operatorname{Int} N(K)$ is an essential annulus in $X\left(r_{a}\right)$ and $\hat{F}_{b}$ is an essential torus in $X\left(r_{b}\right)$, so by [GW1, Theorem 1.1] $X=M_{i}$ for some $i=1,2,3$, and we are done. Hence we may assume that $X$ is non-hyperbolic. $X$ is irreducible as otherwise there would be an essential sphere $S$ in $X$ bounding a 3-ball in $M$ containing $K$, which would be a contradiction to the fact that $K$ is an essential curve on $\hat{F}_{a}$. Also $X$ cannot be a Seifert fibered manifold as otherwise $M=X \cup N(K)$ would be non-hyperbolic. Since by Lemma $21.1 \partial M$ is a union of tori, the above implies that $X$ must be toroidal.

Since $M$ is atoroidal, an essential torus $T$ in $X$ must be separating. Let $T_{0}$ be the Dehn filling torus component of $M$, and let $T_{1}=\partial N(K)$. Recall that $M \mid T$ denotes the manifold obtained by cutting $M$ along $T$. Let $V=V_{T}$ and $W=W_{T}$ be the components of $M \mid T$, where $W$ is the component containing $T_{0}$. Among all essential tori in $X$, choose $T$ so that (a) if there is some $T$ in $X$ such that $T_{1} \subset W_{T}$, choose $T$ so that $V_{T}$ contains no essential torus; (b) if every essential torus in $X$ separates $T_{0}$ from $T_{1}$, choose $T$ such that $W_{T}$ contains no essential torus.

Since $M$ is atoroidal, $T$ is inessential in $M$, hence $V$ is either (i) a solid torus, or (ii) $T^{2} \times I$, or (iii) a 3 -ball with a knotted hole. Note that in the first two cases $V$ must contain the curve $K$. Let $N=V-\operatorname{Int} N(K)$ in the first two cases, and $N=V$ in the last case. Let $C=T \cap F_{a}$. Using a standard cut and past argument we may assume that each component of $C$ is essential on both $T$ and $F_{a}$. In case (iii) let $D$ be a compressing disk of $T$ in $W$.

Claim 1. $C \neq \emptyset$.

Proof. If $C=\emptyset$ then $F_{a}$ lies in $W$, which is impossible in cases (i) or (ii) because the curve $K$ on $F_{a}$ lies in $V$. In case (iii) $D \cap F_{a}$ is a set of circles and one can use the incompressibility of $F_{a}$ in $W$ to isotop $F_{a}$ so that it is disjoint from $D$. But then $D$ is disjoint from $K$, so $T$ would be compressible in $X$, which is a contradiction.

Claim 2. $C$ is a set of essential curves on $\hat{F}_{a}$ parallel to $K$.

Proof. Since $C$ is disjoint from $K$, we need only show that each component $\alpha$ of $C$ is an essential curve on $\hat{F}_{a}$. Assume to the contrary that $\alpha$ bounds a disk $E$ on $\hat{F}_{a}$ and is innermost on $\hat{F}_{a}$. Then $E$ must contain some boundary component of $F_{a}$, hence $E \subset W\left(r_{a}\right)$. In case (i) $V \cup N(E)$ is either a 3-ball, or a punctured lens space or $S^{1} \times S^{2}$, containing the curve $K$, contradicting the fact that $\hat{F}_{a}$ is incompressible and $M\left(r_{a}\right)$ irreducible. In case (ii) $V \cup N(E)$ 
is a punctured solid torus, so the irreducibility of $M\left(r_{a}\right)$ implies that $M\left(r_{a}\right)$ is a solid torus, which is absurd because it is supposed to be toroidal. In case (iii), for homological reasons $\partial E$ and $\partial D$ must be homotopic on $T$, hence $\partial E$ is null-homotopic in $M$, which contradicts the facts that $C$ is essential on $F_{a}$ and $F_{a}$ is incompressible in $M$.

Claim 3. Case (iii) cannot happen, i.e. $V$ is not a 3-ball with a knotted hole.

Proof. We have shown that all components of $C$ are essential curves on $\hat{F}_{a}$ parallel to $K$, and $C \neq \emptyset$. Let $\alpha$ be a component of $C$. Then $K$ is isotopic to $\alpha$ in $M\left(r_{a}\right)$, but since $\alpha \subset T$ lies in the 3-ball $V \cup N(D), \alpha$, and hence $K$, is null-homotopic in $M\left(r_{a}\right)$, which contradicts the fact that $\hat{F}_{a}$ is incompressible in $M\left(r_{a}\right)$.

Claim 4. $W$ is hyperbolic.

Proof. Clearly $W$ is irreducible (since $X$ is) and not a Seifert fibered space (since $M$ is hyperbolic). Suppose $W$ contains an essential torus $T^{\prime}$. By Claim 3 we see that $T^{\prime}$ cannot be of type (iii), so it must be of type (i) or (ii), which, by our choice of $T$, implies that every essential torus in $X$ separates $T_{0}$ from $T_{1}$. By the choice of $T, W$ must be atoroidal.

We now continue with the proof of Lemma 21.2. Let $A$ be a component of $F_{a} \cap W$ which contains some boundary components of $F_{a}$. By Claims 1 and 2, the corresponding component $\hat{A}$ of $\hat{F}_{a} \cap W\left(r_{a}\right)$ is an annulus in $W\left(r_{a}\right)$, which is incompressible because $\hat{F}_{a}$ is incompressible, and not boundary parallel because otherwise $\hat{F}_{a}$ would be isotopic to a torus with fewer intersections with the Dehn filling solid torus. Therefore $W\left(r_{a}\right)$ is annular.

Let $P$ be the component of $F_{a} \cap V$ containing $K$, and let $\beta$ be a component of $P \cap T$. Note that $P$ is an annulus. Since $F_{b}$ is disjoint from $K$, it can be isotoped to be disjoint from $P$, hence after isotopy we may assume that $F_{b} \cap T$ and $F_{a} \cap T$ are all parallel to $\beta$ and hence mutually disjoint. If $F_{b} \cap T=\emptyset$ then $\hat{F}_{b}$ is an essential torus in $W\left(r_{b}\right)$, and if $F_{b} \cap T \neq \emptyset$ then as above, a component of $\hat{F}_{b} \cap W\left(r_{b}\right)$ which intersects the Dehn filling solid torus is an essential annulus in $W\left(r_{b}\right)$, hence $W\left(r_{b}\right)$ is either toroidal or annular. Using Theorem 1.1 of [GW1] in the first case and Theorem 1.1 of [GW3] in the second case, we see that $W=M_{i}$ for $i=1,2$, or 3 .

By Claim $3 V$ is either a solid torus or $T^{2} \times I$. In the first case $M=M_{i}(s)$ for some $s$ on $T_{1}=\partial V$, and in the second case $M=M_{i}$.

Definition 21.3 (1) Define a set of triples $\left(M_{i}, r_{i}^{\prime}, r_{i}^{\prime \prime}\right)$ as follows. For $i=$ $1,2,3,\left(M_{i}, r_{i}^{\prime}, r_{i}^{\prime \prime}\right)$ are the manifolds and the toroidal/annular slopes given in Theorem 1.1 of $[G W 1] . \quad M_{4}, \ldots, M_{14}$ are the manifolds $X\left(F_{1}, F_{2}\right)$ corresponding to the intersection graphs given in Figures 11.9, 11.10, 14.5, 16.6, 16.8, 16.9, 18.2, 19.4, 20.4, 20.5 and 20.6, and $r_{i}^{\prime}, r_{i}^{\prime \prime}$ are the boundary slopes of the corresponding surfaces $F_{1}, F_{2}$. 
(2) Two triples $\left(M, r^{\prime}, r^{\prime \prime}\right)$ and $\left(N, s^{\prime}, s^{\prime \prime}\right)$ are equivalent, denoted by $\left(M, r^{\prime}, r^{\prime \prime}\right) \cong$ $\left(N, s^{\prime}, s^{\prime \prime}\right)$, if there is a homeomorphism from the 3-manifold $M$ to $N$ which sends the boundary slopes $\left(r^{\prime}, r^{\prime \prime}\right)$ to $\left(s^{\prime}, s^{\prime \prime}\right)$ or $\left(s^{\prime \prime}, s^{\prime}\right)$.

The following theorem shows that if a hyperbolic manifold $M$ admits two toroidal Dehn fillings along slopes $r_{1}, r_{2}$ of distance 4 or 5 then $\left(M, r_{1}, r_{2}\right)$ is either one of these triples, or obtained from such an $M_{i}$ by Dehn filling on $\partial M_{i}-T_{0}$.

Theorem 21.4 Let $M$ be a hyperbolic 3-manifold admitting two toroidal Dehn fillings $M\left(r_{1}\right), M\left(r_{2}\right)$ with $\Delta\left(r_{1}, r_{2}\right)=4$ or 5 . Let $n_{a}$ be the minimal number of intersections between essential tori and the Dehn filling solid torus in $M\left(r_{a}\right)$. Assume $n_{a} \leq n_{b}$. Let $\left(M_{i}, r_{i}^{\prime}, r_{i}^{\prime \prime}\right)$ be the manifolds defined above, and let $T_{0}$ be the boundary component of $M_{i}$ containing $r_{i}^{\prime}, r_{i}^{\prime \prime}$. Then

(1) $n_{a} \leq 2, n_{b} \leq 6$;

(2) either $\left(M, r_{1}, r_{2}\right) \cong\left(M_{i}, r_{i}^{\prime}, r_{i}^{\prime \prime}\right)$ for some $i=1, \ldots, 14$, or $\left(M, r_{1}, r_{2}\right) \cong$ $\left(M_{i}(s), r_{i}^{\prime}, r_{i}^{\prime \prime}\right)$, where $i \in\{1,2,3,14\}$ and $s$ is a slope on $T_{1}=\partial M_{i}-T_{0}$; and

(3) $i \in\{1,2,4,6,9,13,14\}$ if $\Delta=4$, and $i \in\{3,5,7,8,10,11,12\}$ if $\Delta=5$.

Proof. This is a summary of the results in the previous sections. Assume $n_{a} \leq n_{b}$. Then by Proposition 11.10 we have $n_{a} \leq 2$. By Proposition 16.8 if $n_{b} \geq 3$ then $X$ is one of those in Figure 11.9, 11.10, 14.5, 16.6, 16.8 or 16.9.

We may now assume $n_{a}, n_{b} \leq 2$. Then by Proposition $20.4 \Gamma_{a}, \Gamma_{b}$ is one of the 11 pairs listed there. One can check that all but cases (3), (5), (6), (9), (10) have the property that one of $\hat{F}_{a}, \hat{F}_{b}$ contains a non-disk face, so by Lemma 21.2 the triple $\left(M, r_{1}, r_{2}\right)$ is $\left(M_{i}, r_{i}^{\prime}, r_{i}^{\prime \prime}\right)$ for some $i=1,2,3$. Finally, by Proposition 20.5 the graphs of the above cases are given in Figures 18.2, 19.4, 20.4, 20.5 and 20.6 .

(3) follows by counting $\Delta$ for the graph pairs of each of the manifolds listed in (2).

\section{The construction of $M_{i}$ as a double branched cover}

The first three of the 14 manifolds $M_{i}$ have already been identified as the exteriors of links in $S^{3}$. See [GW1]. The links are shown in Figure 24.1 Besides $M_{4}$ and $M_{5}$, the other nine manifolds $M_{6}, \ldots, M_{14}$ have the property that $\Gamma_{a}$ is a graph on $\hat{F}_{a}$ with two vertices of opposite signs. In this section we will construct, for each $i=6, \ldots, 14$, a tangle $Q_{i}=\left(W_{i}, K_{i}\right)$, where $W_{i}$ is a 3 -ball for $i=6, \ldots, 13$, and an $S^{2} \times I$ for $i=14$, such that $M_{i}$ is the double branched cover of $W_{i}$ with branch set $K_{i}$. It is well known that once we have such a presentation then the Dehn filling $M_{i}(r)$ will be the double branched cover of $Q_{i}(r)$, where $Q_{i}(r)$ is obtained by attaching a rational tangle of slope $r$ to $Q_{i}$, with coordinates properly chosen. 
Here is a sketch of the construction. Assume $\Gamma_{a}$ is non-positive and $n_{a}=2$, and suppose there is an orientation-preserving involution $\alpha_{1}$ on $F_{a}$ which maps $\partial u_{1}$ to $\partial u_{2}$ and preserves $\Gamma_{a}$. The restriction of $\alpha_{1}$ on $\partial F_{a}$ extends to an involution $\alpha_{2}$ on $T_{0}$ which has four fixed points, and it preserves the curves $\partial F_{b}$ on $T_{0}$. Thus $\alpha=\alpha_{1} \cup \alpha_{2}$ is an involution on $F_{a} \cup T_{0}$, which has eight fixed points, four on each of $F_{a}$ and $T_{0}$. Since $\alpha$ preserves $\Gamma_{a} \cup \partial F_{b}$, it extends over each disk face of $F_{b}$ to give an involution on $F_{b}$. One can now further extend the involution $\alpha$ from $F_{a} \cup F_{b} \cup T_{0}$ to a regular neighborhood $Y$ of $F_{a} \cup F_{b} \cup T_{0}$. For $i \geq 6$, $M_{i}$ is obtained by capping off spherical boundary components of $Y$ by 3-balls, hence $\alpha$ extends to an involution of $M_{i}$. Clearly the quotient of $N\left(F_{a} \cup T_{0}\right)$ is a twice punctured 3-ball $W_{i}$. After attaching 2-handles corresponding to faces of $F_{b}$ and some 3 -balls we see that $W_{i}$ is a punctured 3-ball. From the construction below we will see that $W_{i}$ is a 3 -ball when $i=6, \ldots, 13$, and an $S^{2} \times I$ when $i=14$. Denote by $K_{i}$ the branch set of $\alpha$ in $W_{i}$. Then $Q_{i}=\left(W_{i}, K_{i}\right)$ is the tangle corresponding to the manifold $M_{i}$, and $M_{i}$ is the double branched cover of $Q_{i}$ in the sense that it is the double branched cover of $W_{i}$ with branch set $K_{i}$. Attaching a rational tangle of slope $t$ to $T_{i}$, we obtain a new tangle $Q_{i}(t)$ whose double branched cover is $M(r)$ for some slope $r$ on $T_{0} \subset \partial M$. This makes it possible to see the essential torus in $M_{i}\left(r_{a}\right)$ as a lifting of some surface in $Q_{i}(t)$.

To illustrate this procedure, we give below a step by step construction of the tangle $Q_{6}=\left(W_{6}, K_{6}\right)$ for the manifold $M_{6}$ corresponding to the graphs in Figure 14.5. The constructions for the other manifolds are similar.

Denote by $N(C)$ a regular neighborhood of a set $C$ in a 3-manifold, and by $I$ the interval $[-1,1]$.

STEP 1. Identify $\left[N\left(F_{a} \cup T_{0}\right) / \alpha\right]-D_{2} \times I$ with $S^{2} \times I$.

Recall that $\alpha$ has four branch points on each of $T_{0}$ and $F_{a}$, so $T_{0} / \alpha=S$ is a 2-sphere, and $F_{a} / \alpha=D_{1}$ is a disk. Let $D_{2}$ be a small disk in the interior of $D_{1}$, disjoint from $\Gamma_{b} / \alpha$ and the branch points of $\alpha$. Then $A_{1}=D_{1}-\operatorname{Int}\left(D_{2}\right)$ is a collar of $\partial D_{1}$. Therefore $N\left(\left(F_{a} \cup T_{0}\right) / \alpha\right)$ can be written as

$$
(S \times I) \cup\left(A_{1} \times I\right) \cup\left(D_{2} \times I\right)
$$

Note that $A_{1} \times I$ is a collar of the attaching annulus $\partial D_{1} \times I$, hence $X=$ $(S \times I) \cup\left(A_{1} \times I\right)$ is homeomorphic to $S^{2} \times I$.

One boundary component of $X=S^{2} \times I$ is $\partial_{-} X=\left(T_{0} \times\{-1\}\right) / \alpha$, and the other boundary component $\partial_{+} X$ can be written as $D_{+} \cup D_{-} \cup A$, where the two disks $D_{+} \cup D_{-}=\partial X \cap(S \times 1)$ lift to two annuli on $T_{0} \times 1$, and $A$ is the annulus $\partial X \cap\left(A_{1} \times I\right)$. We identify $X$ with $\left(\mathbb{R}^{2} \cup\{\infty\}\right) \times I$, so that the disks $D_{ \pm}$are identified with the squares $I \times[ \pm 2, \pm 4]$ on the plane $P=\mathbb{R}^{2} \times 1$ on $\partial X$, the annulus $A$ is the closure of $P-D_{+} \cup D_{-}$, and the core $c_{0}$ of $A$ is identified with the closure of the $x$-axis of $P$. See Figure 22.1. (Not drawn to scale.) 


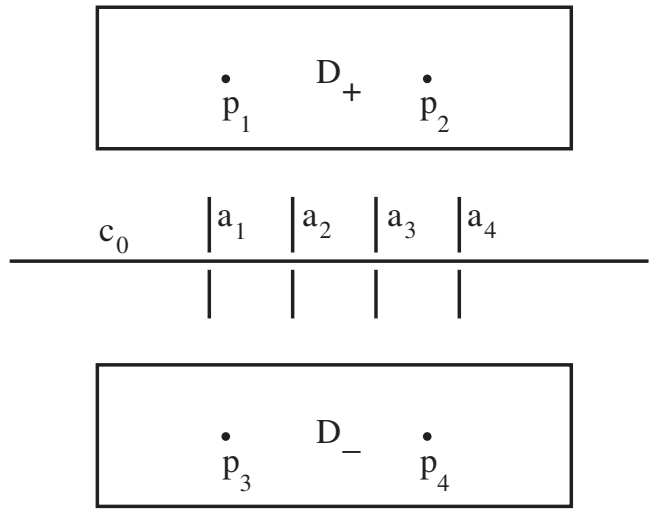

Figure 22.1

The branch set of $\alpha$ now consists of eight arcs. Four of them come from the fixed points of $\alpha$ on $T_{0} \times I$, and are of type $p_{i} \times I$ in $X$, where $p_{1}, p_{2} \in D_{+}$, and $p_{3}, p_{4} \in D_{-}$. These will be represented by four $\operatorname{dots} p_{1}, \ldots, p_{4}$ on $P$, two in each of $D_{ \pm}$, as shown in Figure 22.1. The other four branch arcs of $\alpha$ are of type $a_{i}=q_{i} \times I \subset A \times I$, where $q_{1}, \ldots, q_{4}$ are the branch points of $\alpha$ in $A=D_{1}-\operatorname{Int}\left(D_{2}\right)$. Note that $a_{i}$ has both endpoints on $S^{2} \times 1$. We may assume that these project to four vertical arcs on the annulus $A$ in $P$ above, and we may arrange so that the endpoints of these arcs have $y$-coordinates \pm 1 on the plane $P$. See Figure 22.1. Denote by $a_{i}(1)$ and $a_{i}(-1)$ the endpoints of $a_{i}$ with $y$ coordinates 1 and -1 , respectively.

STEP 2. Draw the arcs $G^{\prime}=\left(\Gamma_{a} \times\{ \pm 1\}\right) / \alpha$ on $P$, with edges and edge endpoints labeled.

The graph $\Gamma_{a} \times 1$ on $F_{a} \times 1$ projects to a set of $\operatorname{arcs} E$ on $D_{1} \times 1$. We may choose the disk $D_{2}$ above to be disjoint from $E$. Then $E$ lies in the annulus $A_{+}=A \times 1$. If a family $\hat{e}_{i}$ has $2 k$ edges then they project to $k$ edges on $A_{+}$ with endpoints on $\partial D_{+}$, each circling around the branch point $a_{i}(1)$. If $\hat{e}_{i}$ has $2 k+1$ edges then the quotient is a set of $k$ edges as above together with an edge connecting a point on $\partial D_{+}$to $a_{i}(1)$. Up to isotopy we may assume that all edge endpoints of $E$ on $\partial D_{+}$lie on the horizontal line $y=2$ on $P$. Similarly the projection of $\Gamma_{a} \times(-1)$ is a set of arcs on the annulus $A_{-}=A \times(-1)$, which is the mirror image of the $\operatorname{arcs}\left(\Gamma_{a} \times 1\right) / \alpha$ along the circle $c_{0}$ on $P$. Denote by $G^{\prime}$ the set of arcs above.

For the graph $\Gamma_{a}$ in Figure 14.5(a), the edges in $G^{\prime}$ are shown in Figure 22.2. The edges are labeled by the corresponding edges in $\Gamma_{a}$. (We only show a few of the labels in the figure; the others should be easy to identify.) Each edge in $G$ is the image of two edges in $\Gamma_{a}$, hence it has two labels. (Note that if one of the families has an odd number of edges then the middle one projects to an arc in $G^{\prime}$ with a single label.) All arcs appear in the region $I \times[-2,2]$. The top and bottom lines in the figure represent $\operatorname{arcs}$ on $\partial D_{ \pm}$. Note that each edge endpoint 
on $\partial D_{+}$corresponds to one edge endpoint on each of $\partial u_{1}$ and $\partial u_{2}$. The labels on the top and bottom lines correspond to the labels on $\partial u_{1}$ in Figure 14.5(a).

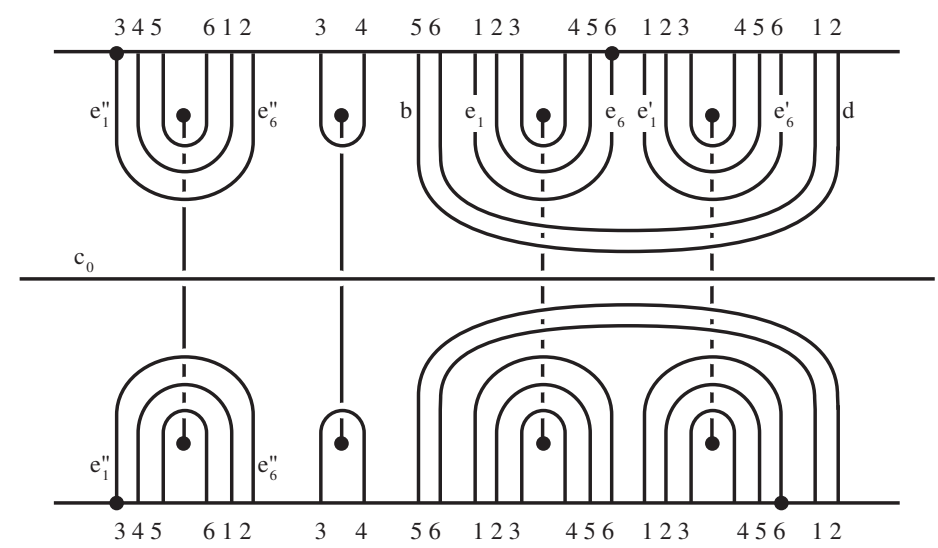

Figure 22.2

STEP 3. Add arcs $G^{\prime \prime}=\left(\partial F_{b} \times 1\right) / \alpha$ on $P$ to obtain $G=G^{\prime} \cup G^{\prime \prime}$.

Recall that the preimage of $D_{ \pm}$are two annuli $A_{ \pm}$on $T_{0} \times 1$. The curves $G^{\prime \prime}=\left(\partial F_{b} \times 1 \cap A_{ \pm}\right) / \alpha$ is now a set of arcs in $D_{ \pm}$, with $\partial G^{\prime \prime}$ the union of $\partial G^{\prime}$ and possibly some of the branch points $p_{i}$ in $D_{ \pm}$. We need to determine how the endpoints of $G^{\prime}$ are connected by the edges of $G^{\prime \prime}$.

Consider the circle $\partial v_{6}$ in Figure 14.5(b). We may assume that the segments on $\partial v_{6}$ from label 1 to label 2 (in the counterclockwise direction) project to arcs in $D_{+}$while those from label 2 to label 1 project to arcs in $D_{-}$. Consider the $\operatorname{arc} \beta$ on $\partial v_{6}$ from the tail of $e_{6}$ to the head of $e_{6}^{\prime \prime}$. (Recall that $e_{6}^{\prime \prime}$ is the edge between $e_{6}$ and $e_{6}^{\prime}$ in Figure 14.5(a)). Note that the tail of $e_{6}$ projects to the endpoint of $e_{1}=e_{6}$ with label 6 on $\partial D_{+}$in Figure 22.2. The other endpoint $q$ of $\beta$ is the head of $e_{6}^{\prime \prime}$, which lies on $\partial u_{2}$. Since the labels in Figure 22.2 are the ones corresponding to those on $\partial u_{1}$ in Figure 14.5(a), we have to find the corresponding point on $\partial u_{1}$ in order to determine the position of the edge endpoint $q$ on $\partial D_{+}$. On Figure 14.5(a) the involution $\alpha$ restricted to $\partial u_{2}$ is a vertical translation, which maps the head of $e_{6}^{\prime \prime}$ (i.e. the edge in $\hat{e}_{3}$ labeled 6 at $u_{2}$ ) to the tail of $e_{1}^{\prime \prime}$, which has label 3 at $u_{1}$. It follows that $q$ is the endpoint of $e_{1}^{\prime \prime}=e_{6}^{\prime \prime}$ in Figure 22.2 with label 3 at $\partial D_{+}$. The two endpoints of $\beta$ are represented by the two dots on the top line in Figure 22.2. Similarly, let $\beta^{\prime}$ be the arc on $\partial v_{6}$ from the head of $e_{6}^{\prime \prime}$ to the tail of $e_{6}^{\prime}$. Then it is an arc in $D_{-}$ with endpoints on the dots at the bottom line in Figure 22.2.

The $\operatorname{arcs} G^{\prime \prime}$ in $D_{ \pm}$are parallel to each other, and they are non-trivial in the sense that none of them cuts off a disk in $D_{ \pm}$that does not contain a branch point of $\alpha$. Therefore the above information completely determines the $\operatorname{arcs} G^{\prime \prime}$ as well as the branch points $p_{1}, \ldots, p_{4}$ of $\alpha$. (Note that if the number of edge 
endpoints between the dots is odd then the middle arc will have an endpoint on a branch point $p_{i}$.) The graph $G=G^{\prime} \cup G^{\prime \prime}$ is now shown in Figure 22.3.

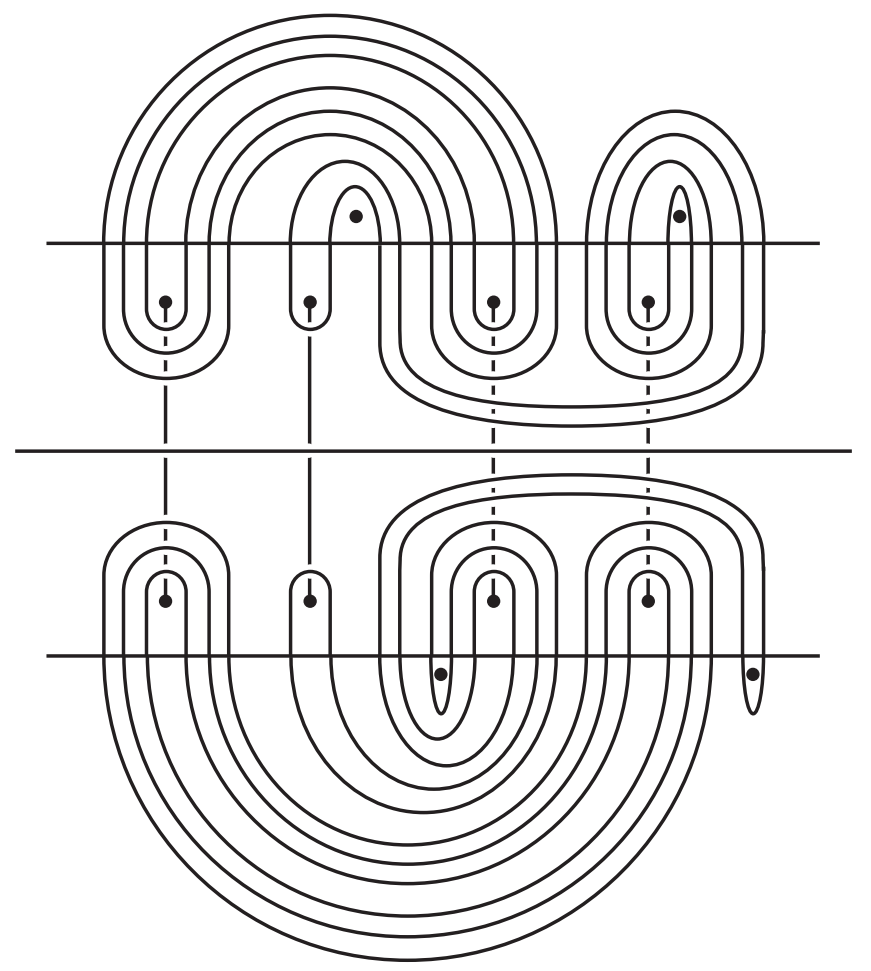

Figure 22.3

STEP 4. Construct the tangle $Q_{i}$.

Each component $c \neq c_{0}$ of $G$ lifts to curves on $F_{b}$ bounding disk faces $\sigma$ of $\Gamma_{b}$. The quotient of $\sigma \times I$ is either a 2-handle attached to $c$ if $c$ is a circle, or a 3-ball attached to a neighborhood of $c$ if $c$ is an arc. Examining the branch set of $\alpha$ in $\sigma \times I$ gives the following procedure. We use $X$ to denote the initial manifold at the beginning of each step below. In particular, $X=S^{2} \times I$ before the first step.

(1) If $c$ is an arc then $X \cup(\sigma \times I) / \alpha$ is homeomorphic to $X$. The new branch set is obtained by adding a trivial arc in $(\sigma \times I) / \alpha$ joining the two endpoints of $c$. Therefore we can simply modify the branch set of $\alpha$ by pushing $c$ into the interior of $X$.

(2) If $c \neq c_{0}$ is a circle component bounding a disk $D_{1}$ on $\partial X$ containing no branch point of $\alpha$, then attaching a 2-handle along $c$ creates a 2-sphere boundary component, which must bound a 3-ball in $M_{i} / \sigma$. Thus after attaching the 
2-handle and the 3-ball the manifold is homeomorphic to $X$, and the homeomorphism maps the new branch set to the old one. Therefore in this case we can simply delete the curve $c$ from $G$.

(3) If $c \neq c_{0}$ is a circle component bounding a disk $D_{1}$ on $\partial X$ containing one branch point of $\alpha$, then $c$ lifts to a circle on the boundary of a face $\sigma$ of $\Gamma_{b}$, which necessarily contains a fixed point of $\alpha$. Hence the cocore of the corresponding 2 -handle is a branch arc of $\alpha$. The 2-sphere boundary component created after attaching the 2-handle contains two branch points of $\alpha$, hence bounds a 3-ball containing a trivial arc as branch set of $\alpha$. Thus after attaching the 2-handle and the 3-ball the manifold is homeomorphic to $X$, and the branch set of $\alpha$ has not changed. As in Case (2), we will simply delete the curve $c$ from $G$ in this case.

(4) If a circle component $c \neq c_{0}$ of $G$ bounds a disk $D_{1}$ containing exactly two branch points of $\alpha$, then after attaching a 2-handle and a 3 -cell, the manifold is homeomorphic to $X$, and the branch set of $\alpha$ is obtained by adding a trivial arc in the 3-cell joining the two branch points of $\alpha$ in $D_{1}$. Therefore in this case we will add an arc in $D_{1}$ joining the two branch points of $\alpha$, push the arc into the interior of $X$ as branch set of $\alpha$, and then delete the curve $c$.

(5) If $c$ is a circle component of $G$ bounding a disk $D_{1}$ containing $k>2$ branch points of $\alpha$, simply attach a 2-handle along $c$. If $k$ is odd, add an arc in the center of the 2-handle to the branch set of $\alpha$.

(6) Finally, attach a 2-handle along $c_{0}$, fill each 2 -sphere boundary component containing at most 2 branch points with a 3-ball, and add a trivial arc in the 3 -ball to the branch set if the 2 -sphere contains exactly two branch points. If the 2-sphere contains four branch point, shrink it by an isotopy to a small sphere, which projects to a small disk on the diagram, with four branch arcs attached. (This happens only for $Q_{14}$. See Figure 22.13.) This completes the construction of the tangle $Q_{i}$.

For $M_{6}$, the above procedure produces the tangle $Q_{6}=\left(W_{6}, K_{6}\right)$ in Figure 22.4(a), where $K_{6}$ should be considered as a tangle lying in the half space $Q_{6}$ (including $\infty$ ) in front of the blackboard. The four boundary points of $K_{6}$ lie on the blackboard, which is the boundary of $W_{6}$. 


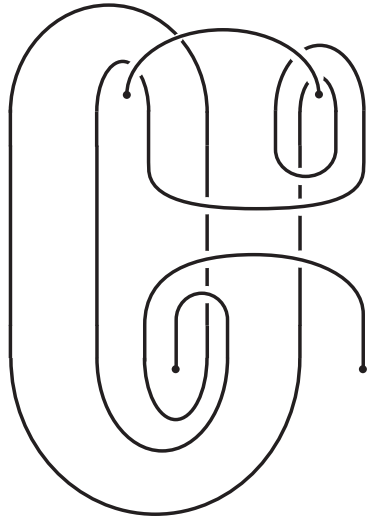

(a)

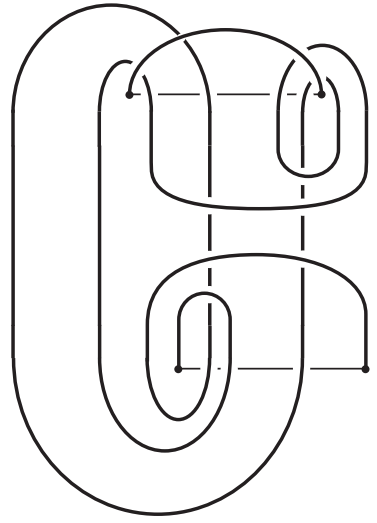

(b)

Figure 22.4

STEP 5. Find the tangles $Q_{i}\left(t_{a}\right)=M_{i}\left(r_{a}\right) / \alpha$.

There is one branch point $p_{i}$ in each quadrant of $P^{\prime}=\mathbb{R}^{2}$. Let $m, l$ be curves on $T_{0}$ that project to the $y$-axis union $\infty$ and the $x$-axis union $\infty$ on $P^{\prime}$, respectively. This sets up coordinate systems on $T_{0}$ and $P^{\prime}$. For $t=p / q$ a rational number or $\infty$, let $M_{i}(t)$ denote the Dehn filling along slope $p m+q l$, and $Q_{i}(t)$ denote the tangle obtained by attaching a rational tangle of slope $t$ to $P^{\prime}$. In other words, $Q_{i}(t)$ is obtained by attaching a 3 -ball to $Q_{i}$ on $P^{\prime}$, and adding two arcs on $P^{\prime}$ connecting the branch points of $\alpha$, which lift to curves of slope $t$ on $T_{0}$. Since the attached rational tangle lifts to a solid torus with meridional slope $t$ on $T_{0}, M_{i}(t)$ is the double branched cover of $Q_{i}(t)$.

By construction $\partial F_{a}$ projects to the $x$-axis, hence $M_{i}\left(r_{1}\right)=M_{i}(0)$. The slope $r_{2}$ can be obtained by connecting the curves $G^{\prime \prime}$ in $D_{ \pm}$by vertical arcs in $A=P^{\prime}-\cup D_{ \pm}$. For $M_{6}$, one can check that the slope $r_{2}=4$.

Denote by $T\left(a_{1}, a_{2}\right)$ a Montesinos tangle which is the sum of two rational tangles of slopes $1 / a_{1}$ and $1 / a_{2}$, respectively, where $a_{1}, a_{2}$ are integers. Denote by $T\left(a_{1}, b_{1} ; a_{2}, b_{2}\right)$ the collection of pairs $\left(S^{3}, L\right)$ which can be obtained by gluing two tangles $T\left(a_{i}, b_{i}\right)$ along their boundary. Denote by $X\left(a_{1}, a_{2}\right)$ the collection of Seifert fiber spaces with orbifold a disk with two cone points $c_{1}, c_{2}$ of index $a_{1}$ and $a_{2}$, i.e. the cone angle at $c_{i}$ is $2 \pi / a_{i}$. Note that the double branched cover of $T\left(a_{1}, a_{2}\right)$ is in $X\left(a_{1}, a_{2}\right)$. Denote by $X\left(a_{1}, b_{1} ; a_{2}, b_{2}\right)$ the collection of graph manifolds which are the union of two manifolds $X_{1}, X_{2}$ glued along their boundary, where $X_{i} \in X\left(a_{i}, b_{i}\right)$.

Denote by $K_{p / q}$ the two bridge knot or link associated to the rational number $p / q$. Denote by $C\left(p_{1}, q_{1} ; p_{2}, q_{2}\right)$ the link obtained by replacing each component $K_{i}$ of a Hopf link by its $\left(p_{i}, q_{i}\right)$ cable $K_{i}^{\prime}$, where $q_{i}$ is the number of times $K_{i}^{\prime}$ winds around $K_{i}$. Denote by $Y\left(p_{1}, q_{1} ; p_{2}, q_{2}\right)$ the double branched cover of $S^{3}$ with branch set $C\left(p_{1}, q_{1} ; p_{2}, q_{2}\right)$. Denote by $C(C ; p, q)$ the link obtained by 
replacing one component $K_{1}$ of a Hopf link by a Whitehead knot in the solid torus $N\left(K_{1}\right)$, and the other component $K_{2}$ by a $(p, q)$ cable of $K_{2}$. Let $Y(C ; p, q)$ be the double branched cover of $S^{3}$ with branch set $C(C ; p, q)$. Denote by $Z$ the double branched cover of $S^{3}$ with branch set the 2-string cable of the trefoil knot shown in Figure 22.12(d).

If $Q_{i}(r)=\left(S^{3}, L\right)$ then we will sometimes simply write $Q_{i}(r)=L$.

Lemma 22.1 (1) $Q_{6}(0) \in T(2,6 ; 2,3)$, as shown in Figure 22.4(b).

(2) $Q_{6}(4)=C(3,1 ; 2,5)$, as shown in Figure 22.5(b).

(3) $Q_{6}(\infty)=K_{9 / 2}$.

Proof. (1) The tangle $Q_{6}(0)=\left(S^{3}, L\right)$ is shown in Figure 22.4(b). A horizontal line at the middle of the diagram corresponds to a 2 -sphere $S$ which cuts the link $L$ into two Montesinos tangles $T(2,6)$ and $T(2,3)$.

(2) The tangle $Q_{6}(4)$ is shown in Figure 22.5(a), which can be isotoped to that in Figure 22.5(b). One can see that it is the link $C(3,1 ; 2,5)$ in $S^{3}$.

(3) The tangle $Q_{6}(\infty)$ is shown in Figure 22.5(c). One can check that it is isotopic to the knot $K_{2 / 9}$ in Figure $22.5(\mathrm{~d})$.

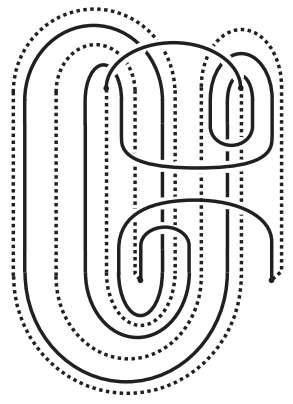

(a)

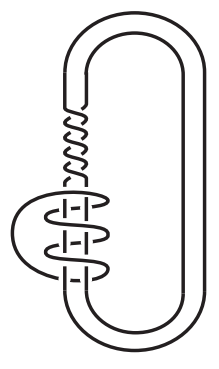

(b)

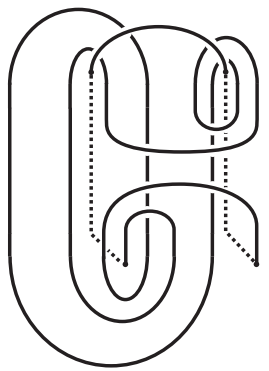

(c)

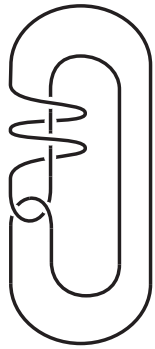

(d)

Figure 22.5

Lemma 22.2 (1) For $i=6, \ldots, 14$, each $M_{i}$ is the double branched cover of a tangle $Q_{i}=\left(W_{i}, K_{i}\right)$, where $Q_{i}$ is shown in Figure 22.4(a) for $i=6$, and in Figure 22.i(b) (with dotted lines removed) when $i>6$.

(2) Each $M_{i}(i=6, \ldots, 13)$ admits a lens space surgery $M_{i}\left(r_{3}\right)$. For each $i$, let $r_{1}, r_{2}$ be the slopes $r_{i}^{\prime}, r_{i}^{\prime \prime}$ given in Definition 21.3. Then the manifolds 
$M_{i}\left(r_{1}\right), M_{i}\left(r_{2}\right)$ and $M_{i}\left(r_{3}\right)$ are given in the following table.

$$
\begin{array}{lll}
M_{6}(0) \in X(2,6 ; 2,3) & M_{6}(4)=Y(3,1 ; 5,2) & M_{6}(\infty)=L(9,2) \\
M_{7}(0) \in X(2,3 ; 3,3) & M_{7}(-5 / 2) \in X(2,3 ; 2,2) & M_{7}(\infty)=L(20,9) \\
M_{8}(0) \in X(2,2 ; 2,6) & M_{8}(-5 / 4)=Y(3,1 ; 2,5) & M_{8}(-1)=L(4,1) \\
M_{9}(0) \in X(2,3 ; 2,3) & M_{9}(-4 / 3)=Y(3,1 ; 2,4) & M_{9}(-1)=L(8,3) \\
M_{10}(0) \in X(2,3 ; 2,3) & M_{10}(-5 / 2)=Y(C ; 2,1) & M_{10}(\infty)=L(14,3) \\
M_{11}(0) \in X(2,4 ; 2,4) & M_{11}(-5 / 2)=Y(C ; 2,1) & M_{11}(\infty)=L(24,5) \\
M_{12}(0) \in X(2,3 ; 2,3) & M_{12}(5)=Y(3,1 ; 2,3) & M_{12}(\infty)=L(3,1) \\
M_{13}(0) \in X(2,3 ; 2,3) & M_{13}(4)=Z & M_{13}(\infty)=L(4,1)
\end{array}
$$

Proof. The result for $M_{6}$ follows from Lemma 22.1 because $M_{6}(r)$ is a branched cover of $Q_{6}(r)$. The proof for the other cases are similar. Each $M_{i}(r)$ is the double branched cover of $Q_{i}(r)$ and the tangle $Q_{i}(r)$ is a link $L$ in $S^{3}$. More explicitly, Figure 22.i(a) shows the curves $G=G^{\prime} \cup G^{\prime \prime}$ in Step 3 of the above construction; Figure 22.i(b) gives the tangle $Q_{i}$ as well as $Q_{i}\left(r_{1}\right)$, which is obtained by attaching a 0 -tangle (the two horizontal dotted lines) to $Q_{i}$; Figure 22.i(c) gives $Q_{i}\left(r_{2}\right)$, which is simplified to that in Figure 22.i(d); $Q_{i}\left(r_{3}\right)$ is in Figure 22.i(e), which is simplified to that in Figure 22.i(f) for some $i$. (The figures are numbered so that Figure 22.i corresponds to the manifold $M_{i}$ for $i \geq 7$. Note that there is no Figure 22.6.) The manifold $M_{14}\left(r_{3}\right)$ is the double branched cover of $Q_{14}\left(r_{3}\right)=T(2,2)$ in Figure 22.14(e), and hence is a twisted $I$-bundle over the Klein bottle. 

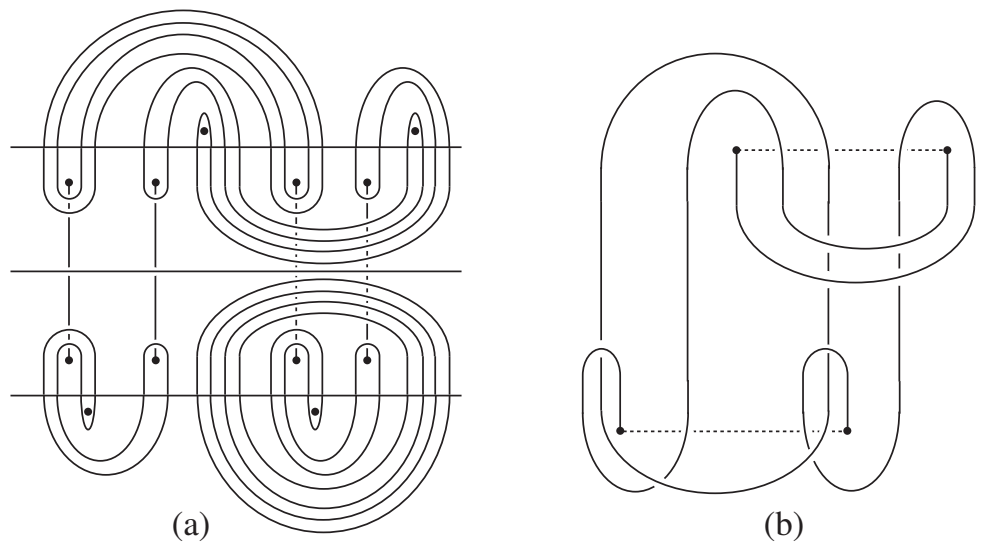

(b)

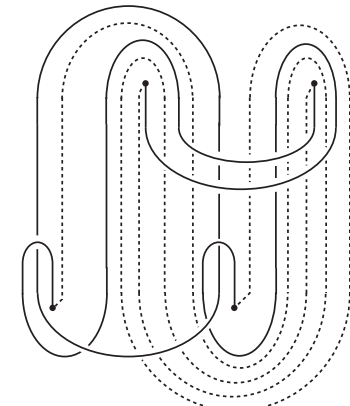

(c)

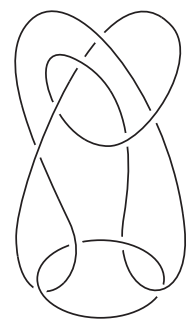

(d)

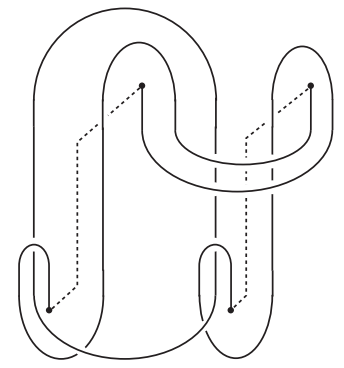

(e)

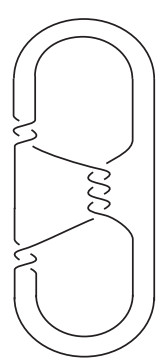

(f)

Figure 22.7 


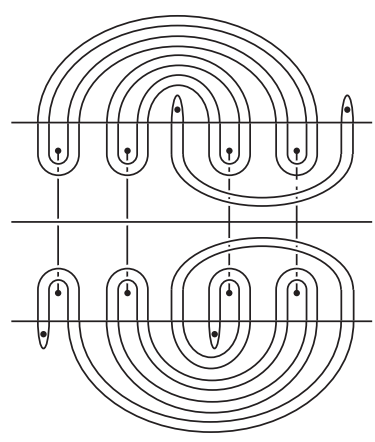

(a)

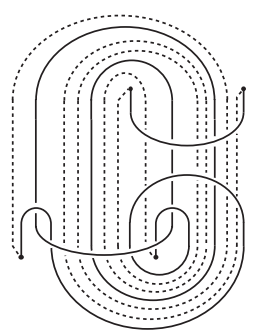

(c)

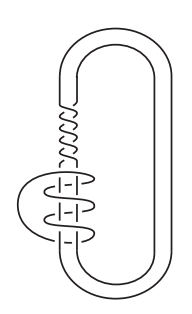

(d)

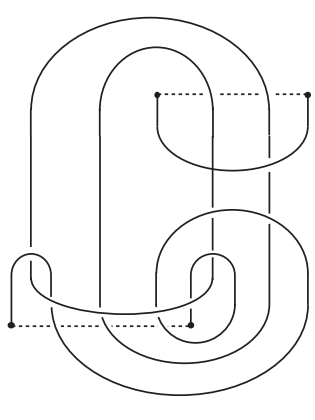

(b)

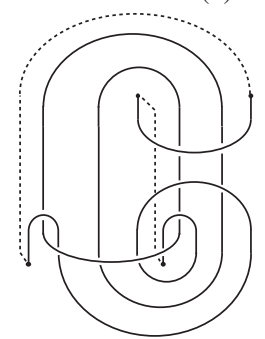

(e)

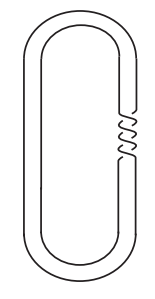

(f)

Figure 22.8

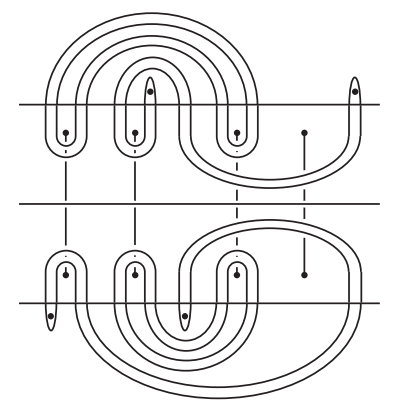

(a)

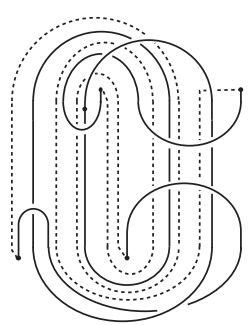

(c)

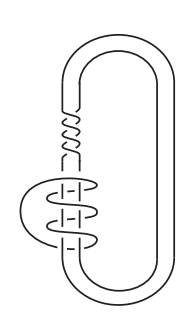

(d)

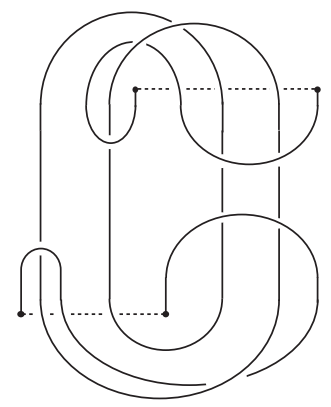

(b)

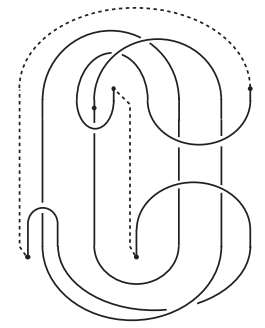

(e)

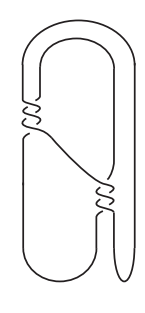

(f) 
Figure 22.9

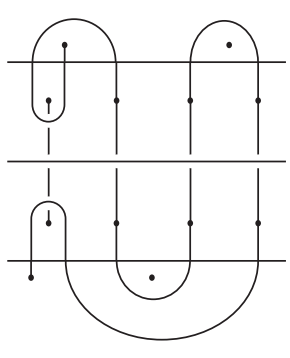

(a)

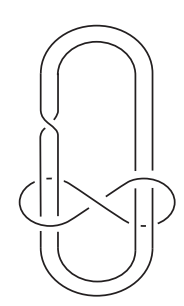

(d)

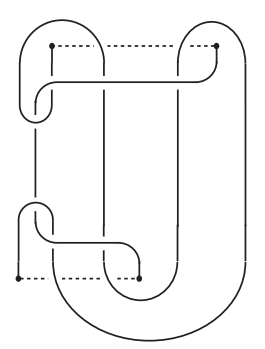

(b)

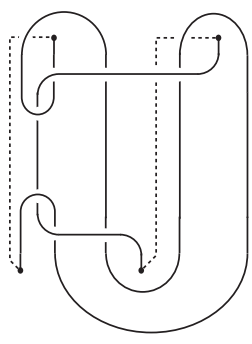

(e)
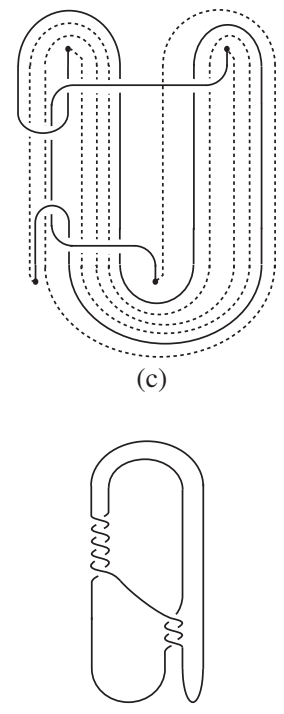

(f)

Figure 22.10

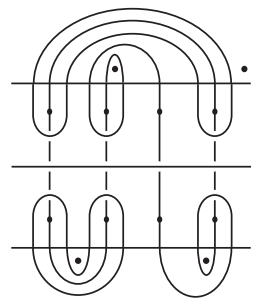

(a)

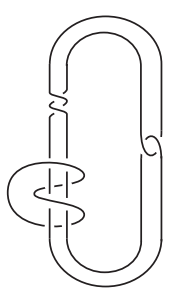

(d)

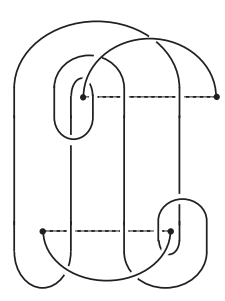

(b)

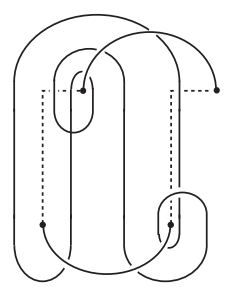

(e)

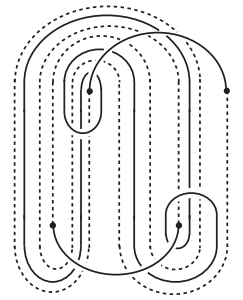

(c)

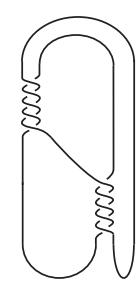

(f)

Figure 22.11 


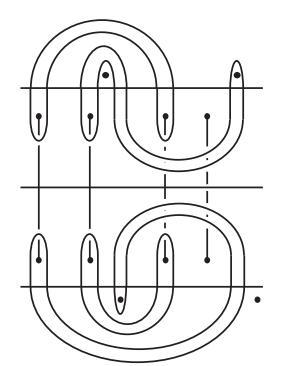

(a)

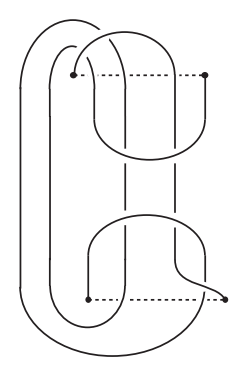

(b)

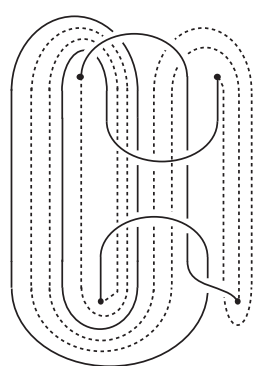

(c)

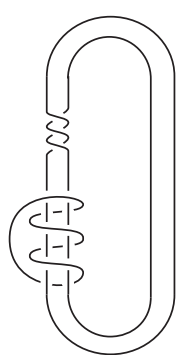

(d)

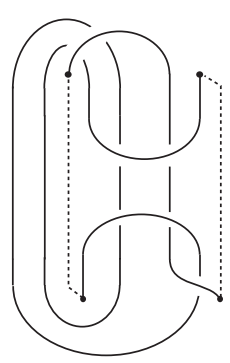

(e)

Figure 22.12

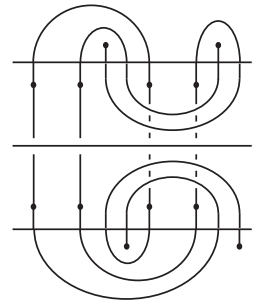

(a)

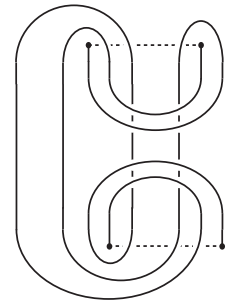

(b)

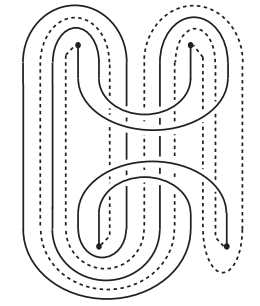

(c)

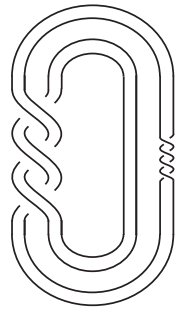

(d)

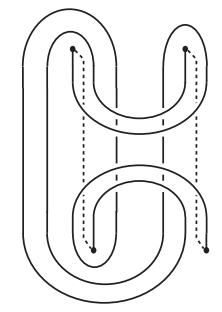

(e)

Figure 22.13

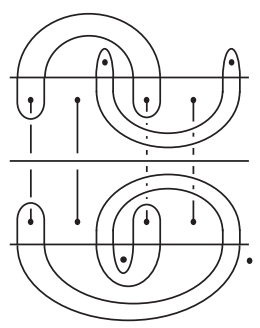

(a)

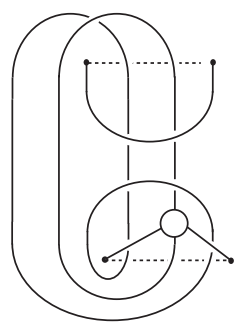

(b)

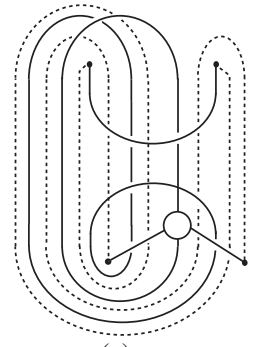

(c)

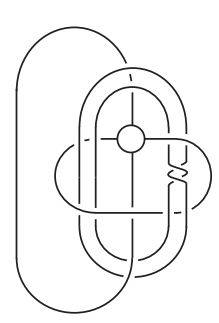

(d)

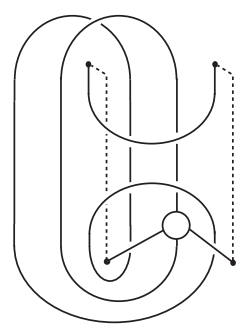

(e)

Figure 22.14

Recall that a manifold $M$ with a fixed torus $T_{0} \subset \partial M$ is large if $H_{2}(M, \partial M-$ $\left.T_{0}\right) \neq 0$. Teragaito [T2] proved that there is no large hyperbolic manifold $M$ admitting two toroidal fillings of distance at least 5 . The following result shows that there is only one such manifold for $\Delta=4$. 
Theorem 22.3 Suppose $\left(M, T_{0}\right)$ is a large manifold and $M$ is hyperbolic and contains two toroidal slopes $r_{1}, r_{2}$ on $T_{0}$ with $\Delta\left(r_{1}, r_{2}\right) \geq 4$. Then $M$ is the Whitehead link exterior, and $\Delta\left(r_{1}, r_{2}\right)=4$.

Proof. Let $r$ be a slope on $T_{0}, V_{r}$ the Dehn filling solid torus in $M(r)$, and $K_{r}$ the core of $V_{r}$. By duality we have $H_{2}\left(M, \partial M-T_{0}\right) \cong H^{1}\left(M, T_{0}\right)$, which is isomorphic to the free part of $H_{1}\left(M, T_{0}\right)$. Also,

$$
H_{1}\left(M, T_{0}\right) \cong H_{1}\left(M(r), V_{r}\right) \cong H_{1}(M(r)) / H_{1}\left(K_{r}\right) .
$$

Put $G(M, r)=H_{1}(M(r)) / H_{1}\left(K_{r}\right)$. Then we need only show that $G\left(M_{i}, r\right)$ is a (possibly trivial) torsion group for $i=2, \ldots, 14$ and $r$ some slope on $T_{0}$.

For $i=2,3, M_{i}$ is the exterior of a closed braid $K_{i}$ in a solid torus $V$. Let $r$ be the meridian slope of $K_{i}$. Then $G\left(M_{i}, r\right)=\mathbb{Z}_{p}$, where $p$ is the winding number of $K_{i}$ in $V$.

For $i=6, \ldots, 13$, by Lemma $22.2 M_{i}$ has a lens space filling $M_{i}\left(r_{3}\right)$. Therefore $G\left(M_{i}, r_{3}\right)$ is a quotient of the finite cyclic group $H_{1}\left(M_{i}\left(r_{3}\right)\right)$ and hence is a torsion group. Similarly for the four manifolds in [Go] with toroidal slopes of distance at least 6 .

For $i=14$, take a regular neighborhood of $u_{1} \cup u_{2} \cup D$ on $\hat{F}_{a}$ as a base point. See Figure 20.6(a). Then $H_{1}\left(M_{14}\left(r_{a}\right)\right)$ is generated by $x, y, s_{1}, s_{2}$, where $x$ is the element of $H_{1}\left(\hat{F}_{a}\right)$ represented by the edges $C$ on Figure 20.6(a), oriented from the label 2 endpoint to the label 1 endpoint, $y$ is represented by $B$, oriented from $u_{1}$ to $u_{2}$, and $s_{i}$ by the part of the core of the Dehn filling solid torus running from $u_{i}$ to $u_{i+1}$ with respect to the orientation of $\partial F_{b}$. Then the bigons $B \cup D$, $C \cup E$ and the 4-gon bounded by $C \cup D \cup E \cup Y$ on $F_{b}$ give relations $2 s_{1}-y=0$, $2 x=0$, and $y+2 x=0$. The other faces of $\Gamma_{b}$ are parallel to these. To calculate $G\left(M_{14}, r_{a}\right)=H_{1}\left(M_{14}\left(r_{a}\right)\right) / H_{1}\left(K_{a}\right)$ we further add the relation $s_{1}+s_{2}=0$. One can now check that $G\left(M_{14}, r_{a}\right)=\mathbb{Z}_{2} \oplus \mathbb{Z}_{2}$, and the result follows.

For $i=4$, choose a regular neighborhood of $v_{1} \cup v_{2} \cup J$ in Figure 11.9(b) as a base point. Then $H_{1}\left(M_{4}\left(r_{b}\right)\right)$ is generated by $x, y, s_{1}, s_{2}$, where $x, y$ are represented by the edges $L, C$ in Figure 11.9(b), oriented from $v_{1}$ to $v_{2}$, and $s_{i}$ by the part of the core of the Dehn filling solid torus from $v_{i}$ to $v_{i+1}$. The faces bounded by $L \cup C, C \cup K$ and $Q \cup K \cup M \cup A$ give the relations $y-s_{1}+x+s_{2}=0$, $s_{1}-x-s_{2}=0$, and $s_{2}-s_{1}+y=0$. Together with the relation $s_{1}+s_{2}=0$ from $H_{1}\left(K_{b}\right)=0$, these give $G\left(M_{4}, r_{b}\right)=\mathbb{Z}_{2}$.

For $i=5, H_{1}\left(M_{5}\left(r_{b}\right)\right)$ is generated by $x, y, s$, where $x, y$ are represented by edges $E$ and $C$ on Figure 11.10(b), oriented from label 3 to label 4 , and $s$ is represented by the core of the Dehn filling solid torus. Then the bigon $A \cup H$ and the annulus bounded by $A \cup G \cup C \cup E$ on Figure 11.10(a) containing $J$ give the relations $x+y=0$ and $2 x-2 y=0$. Adding the relation $s=0$ gives $G\left(M_{5}, r_{b}\right)=\mathbb{Z}_{4}$. 


\section{The manifolds $M_{i}$ are hyperbolic}

The manifolds $M_{1}, M_{2}, M_{3}$ in Definition 21.3 are known to be hyperbolic, see [GW1, Theorem 1.1]. In this section we will show that the other 11 manifolds $M_{i}$ in Definition 21.3 are also hyperbolic. See Theorem 23.14 below.

A knot $K$ in a solid torus $V$ is a $(p, q)$ knot if it is isotopic to a $(p, q)$ curve on $\partial V$ with respect to some longitude-meridian pair on $\partial V$. In particular, the winding number of $K$ in $V$ is $p$.

Lemma 23.1 (1) If $i \in\{6, \ldots, 13\}$ and $j=1$ or $i \in\{6,7,8,9,12,13\}$ and $j=2$, then $M_{i}\left(r_{j}\right)$ contains an essential torus $T$ cutting it into two Seifert fiber spaces $E_{1}, E_{2}$.

(2) For $i=10,11, M_{i}\left(r_{2}\right)$ contains a non-separating essential torus cutting $M_{i}\left(r_{2}\right)$ into a Seifert fiber space whose orbifold is an annulus with a cone point of index 2.

(3) For $i=6, \ldots, 13$ and $j=1,2, M_{i}\left(r_{j}\right)$ is irreducible, and contains no hyperbolic submanifold bounded by an incompressible torus.

Proof. (1) By Lemma 22.2, for $i=6, \ldots, 13, M_{i}\left(r_{1}\right)$ is of type $X\left(a_{1}, b_{1} ; a_{2}, b_{2}\right)$, which is the union of two Seifert fiber spaces of types $X\left(a_{1}, b_{a}\right)$ and $X\left(a_{2}, b_{2}\right)$, hence the result is true for $M_{i}\left(r_{1}\right)$. Similarly it is true for $M_{7}\left(r_{2}\right)$.

For $i=13, Q_{i}\left(r_{2}\right)=\left(S^{3}, L\right)$, where $L$ is the link in Figure 22.13(d), which consists of two parallel copies of the trefoil knot. The two components of $L$ bound an annulus $A$. Cutting $S^{3}$ along $A$ gives the trefoil knot exterior $E$, and $A$ becomes a torus $T$. The double branched cover of $Q_{13}\left(r_{2}\right)$ is obtained by gluing two copies of $E$ along $T$. Hence the result is true because $E$ is a Seifert fiber space and $T$ is incompressible in $E$.

For $i=6,8,9,12, Q_{i}\left(r_{2}\right)=\left(S^{3}, L\right)$ is of type $C\left(p_{1}, q_{1} ; p_{2}, q_{2}\right)$, so there is a torus $T^{\prime}$ cutting $S^{3}$ into two solid tori $V_{1}, V_{2}$, such that each $L_{j}=L \cap V_{j}$ is a $\left(p_{j}, q_{j}\right)$ knot in $V_{j}$ for some $p_{j}>1$. Note also that in these cases at least one of the $p_{j}$ is odd, which implies that $T^{\prime}$ lifts to a single torus $T$, cutting $M_{i}\left(r_{2}\right)$ into two components $W_{1}, W_{2}$, such that $W_{j}$ is a double branched cover of $\left(V_{j}, L_{j}\right)$. The $\left(p_{j}, q_{j}\right)$ fibration of $V_{j}$ now lifts to a Seifert fibration of $W_{j}$, hence the result follows.

(2) For $i=10,11, Q_{i}\left(r_{2}\right)=\left(S^{3}, L\right)$, and there is a torus $T^{\prime}$ cutting $S^{3}$ into two solid tori $V_{1}, V_{2}$, such that $L_{1}=V_{1} \cap L$ is a $(2,1)$ knot, and $L_{2}=V_{2} \cap L$ is a Whitehead knot in the solid torus $V_{2}$. Since both winding numbers of $L_{j}$ are even, $T^{\prime}$ lifts to two tori in $M_{i}\left(r_{2}\right)$. Let $W_{i}$ be the lifting of $V_{i}$. A meridian disk of $V_{1}$ lifts to an annulus in $W_{1}$, hence $W_{1}$ is a $T^{2} \times I$ (not a twisted $I$-bundle over the Klein bottle because $\partial W_{1}$ has two components). Let $T$ be the core of this $T^{2} \times I$. Then it cuts $M_{i}\left(r_{2}\right)$ into the manifold $W_{2}$. We need to show that $W_{2}$ is Seifert fibered.

Let $D$ be a meridian disk of $V_{2}$ which intersects $L_{2}$ at two points. Then $\left(V_{2}, L_{2}\right)=\left(B_{1}, L_{1}^{\prime}\right) \cup\left(B_{2}, L_{2}^{\prime}\right)$, where $B_{1}=N(D), B_{2}$ is the closure of $V_{2}-B_{1}$, and $L_{k}^{\prime}=L_{2} \cap B_{k}$. Note that each $L_{k}^{\prime}$ is a trivial tangle in $B_{k}$, hence its double branched cover $V_{k}^{\prime}$ is a solid torus. One can check that each component 
of $V_{1}^{\prime} \cap V_{2}^{\prime}$ is a longitudinal annulus on $\partial V_{1}^{\prime}$, and it is an annulus on $\partial V_{2}^{\prime}$ with winding number 2 in $V_{2}^{\prime}$. Therefore $W_{2}=V_{1}^{\prime} \cup V_{2}^{\prime}$ is a Seifert fiber space whose orbifold is the union of a $D^{2}$ and a $D^{2}(2)$ glued along two boundary arcs. Since $W_{2}$ has two torus boundary components, the orbifold must be an annulus with a single cone point of index 2 .

(3) Let $T$ be the essential torus in $M_{i}\left(r_{j}\right)$ given in the above proof. Then it cuts $M_{i}\left(r_{j}\right)$ into one or two bounded Seifert fiber spaces, which are irreducible. Since $T$ is incompressible, $M_{i}\left(r_{j}\right)$ is also irreducible. The second statement follows from the fact that the JSJ (Jaco-Shalen-Johannson) decomposition of an irreducible closed 3-manifold is unique.

A $\left(p^{\prime}, q^{\prime}\right)$ knot $K$ in a solid torus $V$ is also called a 0 -bridge knot. In this case there is an essential annulus in $V-\operatorname{Int} N(K)$ with one boundary component in each of $\partial V$ and $\partial N(K)$. This defines a longitude $l$ for $K$, which is unique if $K$ is not the core of $V$. A $(p, q)$ cable of a 0 -bridge knot $K$ is a knot on $\partial N(K)$ which represents $p l+q m$ in $H_{1}(\partial N(K))$, where $m$ is a meridian of $K$. We refer the readers to [Ga1] for the definition of a 1-bridge braid in $V$.

Lemma 23.2 Suppose $X$ is an irreducible, $\partial$-irreducible, compact, orientable 3-manifold with $\partial X=T_{1} \cup T_{0}$ a pair of tori. Let $r_{1}, r_{2}$ be distinct slopes on $T_{0}$ such that $X\left(r_{1}\right), X\left(r_{2}\right)$ are both $\partial$-reducible. Let $K_{a}$ be the core of the Dehn filling solid torus in $X\left(r_{a}\right)$. Then one of the following holds, up to relabeling of $r_{i}$.

(1) Each $X\left(r_{a}\right)$ is a solid torus, $K_{a}$ is a 0 - or 1-bridge braid in $X\left(r_{a}\right)$, and $\Delta\left(r_{1}, r_{2}\right)=1$ if it is not a 0-bridge knot.

(2) $X\left(r_{1}\right)$ is a solid torus, and $X\left(r_{2}\right)=\left(S^{1} \times D^{2}\right) \# L(p, q)$ with $p \geq 2$. $K_{1}$ is a $(p, q)$ cable of $a\left(p^{\prime}, q^{\prime}\right)$ knot in $X\left(r_{1}\right)$, and $r_{2}$ is the cabling slope of $K_{1}$ in $X\left(r_{1}\right)$. Moreover, if $m_{a}$ is the slope on $T_{1}$ bounding a disk in $X\left(r_{a}\right)$, then $\Delta\left(m_{1}, m_{2}\right)=p p^{\prime}$.

Proof. If both $X\left(r_{1}\right), X\left(r_{2}\right)$ are irreducible then they are solid tori and (1) holds by [Ga1, Theorem 1.1] and [Ga2, Lemma 3.2]. Now assume $X\left(r_{2}\right)$ is reducible. Then by [Sch, Theorem 6.1] $K_{1}$ is a $(p, q)$ cable of some knot $K^{\prime}$ in $X\left(r_{1}\right)$ with respect to some meridian-longitude pair $(m, l)$ of $K^{\prime}$, and $r_{2}$ is the cabling slope. In this case $X\left(r_{2}\right)$ is a connected sum $W_{1} \cup L(p, q)$, where $W_{1}$ is obtained by surgery on the knot $K^{\prime}$ in $X\left(r_{a}\right)$ along the cabling slope $r^{\prime}=p l+q m$. Denote by $m^{\prime}$ the meridian slope of $K^{\prime}$. Then $\Delta\left(m^{\prime}, r^{\prime}\right)=p>1$.

Denote by $K^{\prime}(s)$ the manifold obtained by $s$-surgery on $K^{\prime}$ in $V=X\left(r_{1}\right)$. The assumption on $X$ implies that $T_{1}$ is incompressible in $V-K^{\prime}$, and $V-K^{\prime}$ is irreducible. By the above, $T_{1}$ is compressible in both $K^{\prime}\left(m^{\prime}\right)=V$ and $K^{\prime}\left(r^{\prime}\right)=$ $W_{1}$, and $\Delta\left(m^{\prime}, r^{\prime}\right)>1$, hence by [Wu2, Theorem 1.1] and [CGLS, Theorem 2.4.3], either $V-\operatorname{Int} N\left(K^{\prime}\right)=T^{2} \times I$, or there is an annulus $A$ in $V-\operatorname{Int} N\left(K^{\prime}\right)$ with one boundary component on $T_{1}$ and another boundary component a curve of slope $r$ on $T^{\prime}=\partial N\left(K^{\prime}\right)$, satisfying $\Delta\left(r, m^{\prime}\right)=\Delta\left(r, r^{\prime}\right)=1$. In either case $K^{\prime}$ is isotopic to a curve on $T_{1}$ and hence is a 0 -bridge knot. Since $V-K$ is irreducible, this implies that $V$ is also irreducible. Therefore $V=X\left(r_{1}\right)$ is a 
solid torus, $K^{\prime}$ is a $\left(p^{\prime}, q^{\prime}\right)$ knot in $V$ for some $\left(p^{\prime}, q^{\prime}\right)$, and $r$ is the cabling slope of $K^{\prime}$ when $p^{\prime}>1$.

If $p^{\prime}=1$, i.e. $V-\operatorname{Int} N\left(K^{\prime}\right)=T^{2} \times I$, then $K$ is the $(p, q)$ cable of the core $K^{\prime}$ of $V$, and $r_{2}$ is the cabling slope of $K$. We have $X\left(r_{2}\right)=V \# L(p, q)$, and the slope $m_{2}$ on $T_{1}$ which bounds a disk in $X\left(r_{2}\right)$ is the $(p, q)$ curve on $T_{1}$, hence $\Delta\left(m_{1}, m_{2}\right)=p=p p^{\prime}$.

Now assume $p^{\prime}>1$. Choose the longitude $l$ of $K^{\prime}$ to be the cabling slope $r$ of $K^{\prime}$ given above. Since $r^{\prime}=p r+q m$, the equation $\Delta\left(r, r^{\prime}\right)=1$ above implies that $q= \pm 1$. Reversing the orientation of $m^{\prime}$ if necessary we may assume $q=1$. Hence $K$ is a $(p, 1)$ cable of a $\left(p^{\prime}, q^{\prime}\right)$ knot in $V$, and $r_{2}$ is the cabling slope. It is easy to see that the meridian slopes $m_{a}$ of $X\left(r_{a}\right)$ satisfy $\Delta\left(m_{1}, m_{2}\right)=p p^{\prime}$, and the result follows.

Lemma 23.3 Let $i=6, \ldots, 13$. Let $\alpha$ be the covering transformation of the double branched cover $M_{i} \rightarrow Q_{i}$.

(1) $M_{i}$ is irreducible, not Seifert fibered, and contains no non-separating torus.

(2) If $M_{i}$ is not hyperbolic then it contains a separating essential torus $T$ such that $T$ is $\alpha$-equivariant, and the component $W$ of $M_{i} \mid T$ which does not contain $T_{0}$ is either Seifert fibered or hyperbolic.

Proof. (1) If $M_{i}$ is reducible then the summand which does not contain $T_{0}$ is a summand of $M_{i}\left(r_{j}\right)$ for all $r_{j}$, but since $M_{i}\left(r_{3}\right)$ is a lens space while $M_{i}\left(r_{1}\right)$ does not have a lens space summand, this is impossible.

By Lemma 22.2 $M_{i}\left(r_{3}\right)$ is a lens space, so if $M_{i}$ is Seifert fibered then the orbifold of $M_{i}$ is a disk with two cone points, hence $M_{i}\left(r_{1}\right)$ is either a connected sum of two lens spaces or a Seifert fibered space with orbifold a sphere with at most three cone points. This is impossible because by Lemma $22.2 M_{i}\left(r_{1}\right)$ is of type $X\left(a_{1}, b_{1} ; a_{2}, b_{2}\right)$ with some $a_{i}$ or $b_{i}$ greater than 2 , which is irreducible and contains a separating essential torus, at least one side of which is not an I-bundle.

If $M_{i}$ contains a non-separating torus then the lens space $M_{i}\left(r_{3}\right)$ would contain a non-separating surface, which is absurd.

(2) If $M_{i}$ is non-hyperbolic then by (1) it has a non-trivial JSJ decomposition. By $[\mathrm{MeS}]$ we may choose the JSJ decomposition surfaces $F$ to be $\alpha$-equivariant. If we define a graph $G$ with the components of $M_{i} \mid F$ as vertices and the components of $F$ as edges connecting adjacent components of $M_{i} \mid F$, then the fact that $M_{i}$ contains no non-separating torus implies that $G$ is a tree. Let $T$ be a component of $F$ corresponding to an arc incident to a vertex $v$ of valence 1 in $G$. Then $T$ bounds the manifold $W$ corresponding to $v$, which by definition of the JSJ decomposition is either Seifert fibered or hyperbolic.

Lemma 23.4 Suppose $M_{i}$ is non-hyperbolic and let $T$ be the essential torus in $M_{i}$ given in Lemma 23.3(2). Let $X, W$ be the components of $M_{i} \mid T$, where $X \supset T_{0}$. If $T$ is compressible in $M_{i}\left(r_{a}\right)$ for some $a=1,2$, then both $X\left(r_{a}\right)$ and $X\left(r_{3}\right)$ are solid tori, and $W$ is hyperbolic. 
Proof. $T$ is compressible in $X\left(r_{3}\right)$ because $M_{i}\left(r_{3}\right)$ is a lens space. By assumption $T$ is also compressible in $M_{i}\left(r_{a}\right)$. Since $M_{i}\left(r_{a}\right)$ contains no lens space summand, by Lemma 23.2 either both $X\left(r_{a}\right)$ and $X\left(r_{3}\right)$ are solid tori, or $X\left(r_{a}\right)$ is a solid torus and $X\left(r_{3}\right)=\left(S^{1} \times D^{2}\right) \# L(p, q)$ for some $p>1$. We need to show that the second case is impossible.

Let $m_{j}$ be the slope on $T$ which bounds a disk in $X\left(r_{j}\right), j=a, 3$. Then $M_{i}\left(r_{3}\right)=W\left(m_{3}\right) \# L(p, q)$. Since $M_{i}\left(r_{3}\right)$ is a lens space $L(p, q)$, we have $W\left(m_{3}\right)=$ $S^{3}$, so $W$ is the exterior of a knot in $S^{3}$. If $W$ is Seifert fibered then it is the exterior of a torus knot, so $M_{i}\left(r_{a}\right)=W\left(m_{a}\right)$ is obtained by Dehn surgery on a torus knot in $S^{3}$ and hence is atoroidal, which contradicts Lemma 23.1. Since by definition $W$ is Seifert fibered or hyperbolic, this implies that $W$ is hyperbolic. Note that by Lemma $22.2 p \geq 3$ for all $i \in\{6, \ldots, 13\}$. By Lemma 23.2(2) we have $\Delta\left(m_{a}, m_{3}\right) \geq p \geq 3$. Since $W\left(m_{a}\right)=M_{i}\left(r_{a}\right)$ is toroidal and $W\left(m_{3}\right)=S^{3}$, this is a contradiction to [GLu, Theorem 1.1], which says that only integral or half integral surgeries on hyperbolic knots in $S^{3}$ can produce toroidal manifolds. This completes the proof that both $X\left(r_{a}\right)$ and $X\left(r_{3}\right)$ are solid tori.

If $W$ is not hyperbolic then by definition $W$ is Seifert fibered. By the above $X\left(r_{3}\right)$ is a solid torus. Let $m_{3}$ be a meridian slope of $X\left(r_{3}\right)$. Then $M_{i}\left(r_{3}\right)=$ $W\left(m_{3}\right)$, so $M_{i}\left(r_{3}\right)$ being a lens space implies that the orbifold of $W$ is a disk with two cone points, in which case $M_{i}\left(r_{a}\right)=W\left(m_{a}\right)$ is either a connected sum of two lens spaces or a Seifert fiber space with orbifold a sphere with at most three cone points. In the first case $M_{i}\left(r_{a}\right)$ contains no essential torus, while in the second case the only possible essential torus in $M_{i}\left(r_{a}\right)$ is a horizontal torus cutting the manifold into a $T^{2} \times I$, or two twisted $I$-bundles over the Klein bottle. This is a contradiction because by Lemma $23.1 M_{i}\left(r_{a}\right)$ contains an essential torus cutting it into either a Seifert fiber space with orbifold an annulus with a cone point of index 2, or two Seifert fiber spaces, at least one of which is not a twisted $I$-bundle over the Klein bottle.

Lemma 23.5 The torus $T$ in Lemma 23.3(2) is incompressible in both $M_{i}\left(r_{1}\right)$ and $M_{i}\left(r_{2}\right)$.

Proof. First assume that $T$ is compressible in both $X\left(r_{1}\right)$ and $X\left(r_{2}\right)$. By Lemma $23.4 X\left(r_{j}\right)$ is a solid torus for $j=1,2,3$. Since $\Delta\left(r_{1}, r_{2}\right)>1$, by Lemma 23.2 we see that $X$ is the exterior of a $(p, q)$ knot in a solid torus. Since $T$ is not boundary parallel, $p>1$. Let $r$ be the cabling slope on $T_{0}$. Since $X\left(r_{j}\right)$ is a solid torus, we have $r_{j} \neq r$. Therefore by [CGLS, Theorem 2.4.3] we must have $\Delta\left(r, r_{j}\right)=1$ for $i=1,2,3$. By Lemma 23.2 one can check that $\Delta\left(r_{1}, r_{3}\right)=1$ and $\Delta\left(r_{2}, r_{3}\right) \leq 2$. Since $\Delta\left(r_{1}, r_{2}\right) \geq 4$, this is a contradiction because any three slopes $r_{1}, r_{2}, r_{3}$ with distance 1 from a given slope $r$ have the property that $\Delta\left(r_{a}, r_{b}\right)+\Delta\left(r_{b}, r_{c}\right)=\Delta\left(r_{a}, r_{c}\right)$ for some permutation $(a, b, c)$ of $(1,2,3)$.

Now assume that $T$ is compressible in $M_{i}\left(r_{1}\right)$, say. By Lemma $23.4 \mathrm{~W}$ is hyperbolic. On the other hand, by the above $T$ is incompressible in $M_{i}\left(r_{2}\right)$, so $W$ is a submanifold in $M_{i}\left(r_{2}\right)$ bounded by an incompressible torus, hence by Lemma 23.1(3) it is non-hyperbolic, which is a contradiction. 
Lemma 23.6 Let $T\left(a_{1}, b_{1} ; a_{2}, b_{2}\right)=\left(S^{3}, L\right)$, where $a_{i}, b_{i} \geq 2$. If at least one of $a_{1}, b_{1}, a_{2}, b_{2}$ is greater than 2 then the exterior of $L$ is atoroidal, and there is no Möbius band $F$ in $S^{3}$ with $F \cap L$ a component of $L$.

Proof. Denote by $T(a)$ a rational tangle with slope $1 / a$, where $a$ is an integer. Given a tangle $\tau=\left(B^{3}, \tau\right)$, denote $B^{3}-\operatorname{Int} N(\tau)$ by $E$ or $E(\tau)$, and call it the tangle space of $\tau$. Since $T(a)$ is a trivial tangle in the sense that $\tau$ is rel $\partial$ isotopic to arcs on $\partial B^{3}$, the tangle space $E(a)$ is atoroidal, and any incompressible annulus in $B^{3}-\tau$ is trivial in the sense that it is either parallel to an annulus on $\partial\left(B^{3}-\tau\right)$ or cuts off a $D^{2} \times I$ in $B^{3}$ with $\tau \cap\left(D^{2} \times I\right)$ a core arc.

The tangle space $E\left(r_{1}, r_{2}\right)$ of a Montesinos tangle $T\left(r_{1}, r_{2}\right)$ is obtained by gluing $E\left(r_{1}\right), E\left(r_{2}\right)$ along a twice punctured disk $P=E\left(r_{1}\right) \cap E\left(r_{2}\right)$. The above implies that $E\left(r_{1}, r_{2}\right)$ is always atoroidal. If $A$ is an essential annulus in $E\left(r_{1}, r_{2}\right)$ with minimal intersection with $P$, then an innermost circle outermost arc argument shows that $A$ intersects $P$ in essential arcs or circles in $A$. If the intersection is a set of circles then each component of $A \cap E\left(r_{i}\right)$ is a set of trivial annuli, which implies that $A$ is also trivial. If each component of $A \cap P$ is an essential arc then each component of $A \cap E\left(r_{i}\right)$ is a bigon in the sense that it is a disk intersecting $P$ in two arcs, which implies that $r_{i}=2$ for $i=1,2$. Therefore $E\left(r_{1}, r_{2}\right)$ contains no essential annulus unless $r_{1}=r_{2}=1 / 2 \bmod 1$.

By definition $T\left(a_{1}, b_{1} ; a_{2}, b_{2}\right)$ is the union of two Montesinos tangles $T\left(a_{i}, b_{i}\right)$. If the tangle space of $T\left(a_{1}, b_{1} ; a_{2}, b_{2}\right)$ is toroidal then either one of the $T\left(a_{i}, b_{i}\right)$ is toroidal or they are both annular. By the above neither case is possible if at least one of the $a_{1}, b_{1}, a_{2}, b_{2}$ is greater than 2 .

The proof for a Möbius band is similar. If $F$ is a Möbius band in $S^{3}$ bounded by a component of $L$ and has interior disjoint from $L$ then after cutting along the surface $P_{1}=E\left(a_{1}, b_{1}\right) \cap E\left(a_{2}, b_{2}\right)$ it either lies in one of the $E\left(a_{i}, b_{i}\right)$ or intersects each in bigons. One can show that the first case is impossible, and in the second case $a_{i}=b_{i}=2$ for $i=1,2$.

Lemma 23.7 $M_{i}$ is hyperbolic for $i=6$ or $8 \leq i \leq 13$.

Proof. Let $T$ be the $\alpha$-equivariant essential torus in $M_{i}$ given in Lemma 23.3(2). By Lemma $23.5 T$ is incompressible in both $M_{i}\left(r_{a}\right), a=1,2$. Since $T$ is $\alpha$ equivariant, its image $F$ in $Q_{i}=M_{i} / \alpha$ is a 2-dimensional orbifold with zero orbifold Euler characteristic (see [Sct] for definition), and all the cone points have indices 2 . Hence it is $T^{2}, K^{2}, P^{2}(2,2), S^{2}(2,2,2,2), A^{2}, M^{2}$, or $D^{2}(2,2)$, where the surfaces are torus, Klein bottle, projective plane, sphere, annulus, Möbius band and disk, and the numbers indicate the indices of the cone points. Note that in the last three cases the boundary of the surface is part of the branch set of $\alpha$. Since $T$ is incompressible in $M_{i}\left(r_{a}\right), F$ is incompressible in $Q_{i}\left(r_{a}\right)$ in the sense that if some simple loop on $F$ bounds a disk in $Q_{i}\left(r_{a}\right)$ intersecting the branch set at most once then it bounds such a disk on $F$. We need to show that for each type of surface above there is some $a=1,2$ such that no such incompressible 2-dimensional orbifold exists in $Q_{i}\left(r_{a}\right)$. 
We have $Q_{i}=\left(B^{3}, K_{i}\right)$, where $B^{3}=M_{i} / \alpha$ is a 3 -ball and $K_{i}$ is the branch set of $\alpha$. Since $F$ lies in $B^{3}$, it cannot be $K^{2}$ or $P^{2}$. For all $i$ one can check that the branch set $K_{i}$ of $\alpha$ in $Q_{i}$ contains at most one closed circle, hence the case $A^{2}$ is also impossible.

By Lemma 22.2, $Q_{i}\left(r_{1}\right)=T\left(a_{1}, b_{1} ; a_{2}, b_{2}\right)$ for some $a_{j}, b_{j} \geq 2$, and $\left(a_{j}, b_{j}\right) \neq$ $(2,2)$ for some $j$. Therefore by Lemma 23.6 we have $F \neq T^{2}, M^{2}$ for $i=6, \ldots, 13$ because there is no such surfaces in $Q_{i}\left(r_{1}\right)$. It remains to show that $F \neq D^{2}(2,2)$ or $S^{2}(2,2,2,2)$.

For $i=13, Q_{i}\left(r_{2}\right)=\left(S^{3}, L\right)$, where $L$ consists of two parallel copies of a trefoil knot $K$. Since each component of $L$ is non-trivial in $S^{3}, F \neq D^{2}(2,2)$ in this case. Suppose $F=S^{2}(2,2,2,2)$, and let $V$ be a regular neighborhood of the trefoil knot containing $L$, intersecting $F$ minimally. Then $F \cap V \neq \emptyset$, and $F$ is not contained in $V$ as otherwise one can show that $F-L$ would be compressible. Therefore $F \cap V$ is a union of two meridian disks, and $F \cap S^{3}-\operatorname{Int} V$ is an essential annulus in $S^{3}-\operatorname{Int} V$. Since $S^{3}-\operatorname{Int} V$ contains no essential annulus with the meridian of $V$ as boundary slope, this is a contradiction.

The proofs for the cases $i \in\{6,8,9,10,11,12\}$ are similar. In these cases $Q_{i}\left(r_{2}\right)=\left(S^{3}, L\right)$, and there is a torus $T^{\prime}$ cutting $S^{3}$ into two solid tori $V_{1}, V_{2}$, each containing some components of $L$. One can check that no component $L^{\prime}$ of $L$ bounds a disk intersecting $L-L^{\prime}$ at two points, so $F \neq D^{2}(2,2)$. If $F=S^{2}(2,2,2,2)$ then either $F$ lies in one of the $V_{j}$, or it intersects one of the $V_{j}$ in two meridional disks and the other $V_{k}$ in an essential annulus with boundary slope the meridional slope of $V_{j}$. Neither case is possible for the $Q_{i}\left(r_{2}\right)$ listed in Lemma 22.2.

\section{Lemma 23.8 $M_{7}$ is hyperbolic.}

Proof. By Lemma 22.2 we have $M_{7}\left(r_{1}\right) \in X(3,3 ; 2,3)$ and $M_{7}\left(r_{2}\right) \in X(2,2 ; 2,3)$. Consider the tangle decomposition sphere $P_{a}$ of the orbifold $Q_{7}\left(r_{a}\right), a=1,2$, which corresponds to a horizontal plane in Figure 22.7(b), (d) respectively. It lifts to an essential torus $T_{a}$ in $M_{7}\left(r_{a}\right)$.

Each side of $P_{a}$ is a Montesinos tangle of type $T\left(r_{1}, r_{2}\right)$, which is the sum of two rational tangles over a disk $D$. The boundary of $D$ determines the fibration of the double branched cover $X\left(r_{1}, r_{2}\right)$ of $T\left(r_{1}, r_{2}\right)$, which has a unique Seifert fibration unless $r_{1}=r_{2}=2$, in which case the closed circle in the tangle is isotopic (without crossing the arcs) to a curve on the punctured sphere, which lifts to a fiber in the other fibration of $X\left(r_{1}, r_{2}\right)$. It is easy to check from Figures 22.7(b) and (d) that the fiber curves from the two sides of $P_{a}$ do not match, so $M_{7}\left(r_{a}\right)$ is not a Seifert fiber space. Since each side of $T_{a}$ is a small Seifert fiber space with orbifold a disk with two cone points, it follows that $M_{7}\left(r_{a}\right)$ contains no other essential torus.

Suppose $M_{7}$ is non-hyperbolic and let $T$ be the essential torus in $M_{7}$ given by Lemma 23.3. By Lemma 23.5 it is incompressible in both $M_{7}\left(r_{a}\right)$, therefore by the uniqueness of $T_{a}$ above we see that $T=T_{a}$ in $M_{7}\left(r_{a}\right)$ up to isotopy. As before, denote by $W$ and $X$ the components of $M_{7} \mid T$, with $X \supset T_{0}$. Then $W$ is 
the manifold on one side of $T_{a}$ in $M_{7}\left(r_{a}\right)$. Therefore we must have $W=X(2,3)$, so $X\left(r_{1}\right)=X(3,3)$ and $X\left(r_{2}\right)=X(2,2)$. We will show that this is impossible.

Let $Y$ be the component of the JSJ decomposition of $X$ that contains $T$. Then $Y$ is either hyperbolic or Seifert fibered. There are three cases.

Case 1. $T_{0} \subset \partial Y$ and $Y$ is Seifert fibered. By Lemma 23.5 $T$ is incompressible in $Y\left(r_{a}\right)$ for $a=1,2$, so $r_{a}$ is not the fiber slope on $T_{0}$. Hence the Seifert fibration extends over $Y\left(r_{1}\right)$ and $Y\left(r_{2}\right)$. In this case $\partial Y-T_{0}$ is incompressible in $Y\left(r_{a}\right)$. Since $X\left(r_{a}\right)$ is atoroidal, either $Y\left(r_{1}\right) \cong Y\left(r_{2}\right) \cong T^{2} \times I$, or $Y=X$. In the first case we have $X\left(r_{1}\right)=X\left(r_{2}\right)$, which is a contradiction because $X\left(r_{1}\right)=X(3,3) \neq X(2,2)=X\left(r_{2}\right)$. In the second case, Since $X\left(r_{1}\right)$ has orbifold $D^{2}(3,3)$, the orbifold of $X$ must be $A^{2}(3,3)$ or $A^{2}(3)$. On the other hand, since $X\left(r_{2}\right)$ has orbifold $D^{2}(2,2)$, the orbifold of $X$ must be $A^{2}(2,2)$ or $A^{2}(2)$, which contradicts the fact that Seifert fibrations for these manifolds are unique.

Case 2. $T_{0} \subset \partial Y$ and $Y$ is hyperbolic. If $\partial Y$ has more than two boundary components then the fact that $X\left(r_{a}\right)$ is atoroidal implies that $Y\left(r_{a}\right)$ is either $\partial$-reducible, or a $T^{2} \times I$. If $\partial Y=T \cup T_{0}$ then $Y=X$ and by assumption both $Y\left(r_{a}\right)=X\left(r_{a}\right)$ are annular and atoroidal. In either case $Y\left(r_{a}\right)$ is either $\partial$-reducible or annular and atoroidal. Since $\Delta\left(r_{1}, r_{2}\right)=5$ and $Y$ is hyperbolic, this is a contradiction to [Wu2, Theorem 1.1] if both $Y\left(r_{a}\right)$ are $\partial$-reducible, to [GW2] if one of them is $\partial$-reducible and the other is annular, and to [GW3] if both $Y\left(r_{a}\right)$ are annular and atoroidal. (The main theorem of [GW3] said that if $\Delta=5$ then $Y$ and $r_{1}, r_{2}$ are listed in one of the three possibilities in [GW3, Theorem 1.1], but in that case both $Y\left(r_{a}\right)$ are toroidal.)

Case 3. $T_{0} \not \subset \partial Y$. Let $X_{1}$ be the component of $X \mid(\partial Y)$ containing $T_{0}$, and let $X_{2}$ be the closure of $X-X_{1}$, so $X=X_{1} \cup X_{2}$. Since $M_{i}$ contains no nonseparating torus (Lemma 23.3), $T_{1}=X_{1} \cap X_{2}$ is a single torus. Since $X\left(r_{a}\right)$ is atoroidal, $T_{1}$ must be compressible in $X_{1}\left(r_{a}\right)$ for $a=1,2$. Thus Lemma 23.2 and the fact that $X\left(r_{a}\right)$ contains no lens space summand implies that $X_{1}\left(r_{a}\right)$ is a solid torus for $a=1,2$, as in Lemma 23.2(1); moreover, since $\Delta\left(r_{1}, r_{2}\right)>1$, by Lemma 23.2(1) $X_{1}$ is a $(p, q)$ cable space, and by [CGLS, Theorem 2.4.3] $\Delta\left(r, r_{a}\right)=1$ for $r$ the cabling slope. It is easy to see that the meridian slopes $m_{a}$ of $X_{1}\left(r_{a}\right)$ satisfies $\Delta\left(m_{1}, m_{2}\right)=p \Delta\left(r_{1}, r_{2}\right) \geq 5$. Now $X\left(r_{a}\right)=X_{2}\left(m_{a}\right)$, so by Cases 1 and 2 above applied to $X_{2}$ we see that this case is also impossible.

Denote by $c \cdot d$ the minimal intersection number between the two isotopy classes of simple closed curves on a surface represented by $c$ and $d$, respectively. If $\varphi: F \rightarrow F$ is a homeomorphism and $K$ a curve on the surface $F$, then $K$ is said to be $\varphi$-full if for any essential curve $c$ on $F$ there is some $i$ such that $c \cdot \varphi^{i}(K) \neq 0$.

If $K$ is a knot in a 3-manifold $Y$ with a preferred meridian-longitude, denote by $Y(K, p / q)$ the manifold obtained from $Y$ by $p / q$ surgery on $K$. Let $X=$ $F \times I / \psi$ be an $F$-bundle over $S^{1}$ with gluing map $\psi$, let $F_{t}=F \times t, t \in I=[0,1]$, and let $K$ be an essential curve on $F_{1 / 2}$. Then there is a preferred meridianlongitude pair $(m, l)$ on $\partial N(K)$, with $l$ the slope of $F_{1 / 2} \cap \partial N(K)$. 
Lemma 23.9 Let $X=F \times I / \psi$. Let $\eta: F \times I \rightarrow F_{0}$ be the projection, $\varphi=\eta \circ \psi$, and $K$ an essential curve on $F_{1 / 2}$. If $\eta(K) \subset F_{0}$ is $\varphi$-full and $q>1$, then $X(K, p / q)$ is hyperbolic.

Proof. Let $A_{i}$ be an annulus in $X$ with $\partial A_{i}=K \cup K_{i}$, where $i=0,1$ and $K_{i} \subset F_{i}$. Let $V_{i}$ be a regular neighborhood of $A_{i}$. Put $Y=F \times I$. Then $Y=V_{1} \cup W$, where $W$ is homeomorphic to $F \times I$, and $V_{1} \cap W$ is an annulus $A^{\prime}$. After $p / q$ surgery on $K$ we have $Y(K, p / q)=V_{1}(K, p / q) \cup W$. Note that $V(K, p / q)$ is a solid torus with $A^{\prime}$ an annulus on $\partial V_{1}(K, p / q)$ running $q$ times along the longitude. By an innermost circle outermost arc argument one can show that $Y(K, p / q)$ is irreducible, $\partial$-irreducible, atoroidal, and any essential annulus $A_{2}$ can be isotoped to be disjoint from $K_{1}$, i.e. $\partial A_{2} \cdot K_{1}=0$. Moreover, if $A_{2}$ has at least one boundary component on $F_{1}$ then $A_{2}$ is either vertical in the sense that it is isotopic to $c \times I \subset F \times I$ for some curve $c \subset F$, or isotopic to $A^{\prime}$ and hence has both boundary curves parallel to $K_{1}$. Similarly, using $A_{0}$ and $V_{0}$ one can show that $\partial A_{2} \cdot K_{0}=0$, and if $A_{2}$ is not vertical and $A_{2} \cap F_{0} \neq \emptyset$ then it has both boundary curves parallel to $K_{0}$.

The above facts imply that $X(K, p / q)=Y(K, p / q) / \psi$ is irreducible. Since the non-separating surface $F_{0}$ cuts $X(K, p / q)$ into $Y(K, p / q)$, which is not an $I$-bundle, we see that $X(K, p / q)$ is not Seifert fibered. It remains to show that $X(K, p / q)$ is atoroidal.

If $T$ is an essential torus in $X(K, p / q)$ then it can be isotoped so that $T \cap$ $Y(K, p / q)=Q$ is a set of essential annuli. Let $C_{i}=Q \cap F_{i}$. We claim that for any curve $c \subset C_{0}, \varphi(c)$ is isotopic to a curve in $C_{0}$.

We have $\psi\left(C_{0}\right)=C_{1}$, so $\psi(c) \subset C_{1}$. If $\psi(c)$ belongs to a vertical annulus $Q_{1}$ then $\varphi(c)=\eta(\psi(c)) \cong Q_{1} \cap F_{0} \subset C_{0}$. If $\psi(c)$ belongs to a non-vertical annulus then by the property proved above, $\psi(c)$ is isotopic to $K_{1}$, so $\varphi(c) \cong \eta\left(K_{1}\right)=$ $K_{0}$. Note that if $Q$ has a non-vertical component with boundary on $F_{1}$ then the fact that $C_{0}, C_{1}$ have the same number of components implies that there is also a non-vertical component $Q_{0}$ with boundary on $F_{0}$, and we have shown that each component $c^{\prime}$ of $\partial Q_{0}$ is isotopic to $K_{0}$, so $\varphi(c) \cong c^{\prime} \subset \partial Q_{0} \subset C_{0}$. This completes the proof of the claim.

Let $c$ be a component of $C_{0}$. We have shown above that $c \cdot K_{0}=0$ for any $c \subset C_{0}$. Applying the above to $\varphi^{-1}$ we see that there is a curve $c^{\prime} \subset C_{0}$ such that $\varphi\left(c^{\prime}\right) \cong c$. By induction we have $c \cdot \varphi^{i}\left(K_{0}\right)=c^{\prime} \cdot \varphi^{i-1}\left(K_{0}\right)=0$ for all $i$, which is a contradiction to the assumption that $K_{0} \cong \eta(K)$ is $\varphi$-full and hence $c \cdot \varphi^{i}\left(K_{0}\right) \neq 0$ for some $i$.

Lemma 23.10 The manifold $M_{5}$ is hyperbolic.

Proof. Let $W=M_{5} \mid F_{b}$, let $F_{+}, F_{-}$be the two copies of $F_{b}$ in $W$, and let $A$ be the annulus $T_{0} \mid \partial F_{b}$. Then $W$ is obtained from $Y=F_{+} \cup F_{-} \cup A$ by attaching faces of $\Gamma_{a}$ and then some 3 -cells.

Two faces of $\Gamma_{a}$ are parallel if their boundary curves are parallel on $Y$. Since parallel faces cobound a 3-cell in $W$, we need only attach one such face among a set of parallel faces. From Figure 11.10 one can check that the four bigons 
are parallel faces, and the two 6-gons are parallel to each other. Therefore $W$ is obtained from $Y$ by attaching one bigon $\sigma_{1}$, one 6-gon $\sigma_{2}$ and then a 3-cell. Let $\sigma_{1}$ be the bigon on Figure 11.10 between the edges $B$ and $G$, and assume that the edge $B \subset F_{+}$.

Cutting $W$ along $\sigma_{1}$, we obtain a manifold $W_{1}$ with boundary a torus, and it contains the 6-gon $\sigma_{2}$. Therefore it is a solid torus such that the remnant of $F_{+}$, denoted by $F_{+}^{\prime}$, runs along the longitude three times. If we replace $\sigma_{2}$ and the attached 3 -cell by a solid torus $J$ with meridian intersecting $F_{+}^{\prime}$ in one essential arc then $W_{1}$ becomes a $F_{+}^{\prime} \times I$ and $W$ becomes $X=F_{+} \times I$. Therefore $W=X(K, p / q)$, where $K$ is the core of $J$, and $q=3$. Let $\psi: F_{-} \rightarrow F_{+}$be the gluing map, $\eta: F_{+} \times I \rightarrow F_{-}$the projection, and $\varphi=\eta \circ \psi$. By Lemma 23.9 we need only show that the curve $K_{-}$on $F_{-}$isotopic to $K$ is $\varphi$-full.

In $M_{5}$ the bigon $\sigma_{1}$ has boundary edges $B \cup G$ on $F_{b}$, as shown in Figure 11.10(b). Suppose $B \subset F_{+}$and $G \subset F_{-}$when we consider $\sigma_{1}$ as a bigon in $F_{+} \times I$. Then $\psi$ maps the curve $B$ on $F_{-}$to the curve $B$ on $F_{+}$, which is mapped to $G$ on $F_{-}$by $\eta$. Therefore $\varphi: F_{-} \rightarrow F_{-}$maps $B$ to $G$. Since $F_{-} \mid B$ is an annulus and $B$ is disjoint from the curve $K_{-}$above, this determines $K_{-}$. Also $\varphi\left(K_{-}\right)$is the curve on $F_{-}$disjoint from $\varphi(B)=G$, so $K_{-}$intersects $\varphi\left(K_{-}\right)$transversely at a single point, cutting $F_{-}$into an annulus. Therefore $K_{-}$is $\varphi$-full, and the result follows.

Lemma 23.11 The manifold $M_{4}$ is hyperbolic.

Proof. The proof is similar to that of Lemma 23.10. In this case $W=M_{4} \mid F_{b}$ is obtained from $F_{+} \cup F_{-} \cup A_{1} \cup A_{2}$ by attaching two bigons $\sigma_{1}, \sigma_{2}$ and one 4-gon $\sigma_{3}$, so $W=X(K, p / q)$ with $q=2$, where $X=F_{+} \times I$ and $K$ is disjoint from $\sigma_{1}, \sigma_{2}$. Choose $\sigma_{1}, \sigma_{2}$ to be the bigons in Figure 11.9(a) bounded by $H \cup E$ and $E \cup N$, respectively. Then $F_{-}$can be identified with $F_{b}$, and $\sigma_{1}, \sigma_{2}$ intersects $F_{-}$ in the edges $E$ and $N$, respectively. These cut $F_{-}$into an annulus containing the curve $K_{-}$isotopic to the knot $K$ in $X=F_{+} \times I$. The map $\varphi: F_{-} \rightarrow F_{-}$ maps the edges $E$ and $N$ in Figure 11.9(b) to $H$ and $E$, respectively, so $\varphi\left(K_{-}\right)$ is the curve in the annulus $F_{-} \mid(H \cup E)$. The curves $K_{-}$and $\varphi\left(K_{-}\right)$intersect transversely at a single point, cutting $F_{-}$into a neighborhood of $\partial F_{-}$, hence $K_{-}$is $\varphi$-full, and $M_{4}$ is hyperbolic by Lemma 23.9 .

Lemma 23.12 Let $F$ be a closed orientable surface of genus 2, and let $\alpha, \beta$ be two non-separating simple closed curves on $F$, intersecting minimally, cutting $F$ into disks. Let $X$ be obtained from $F \times I(I=[0,1])$ by attaching a 2-handle along $\alpha \times\{0\}$. Identify $F$ with $F \times\{1\} \subset F \times I$, and let $T=\partial X-F$. Then

(1) a compressing disk $D$ of $F$ intersects $\beta$ at least 3 times; and

(2) an incompressible annulus $A$ in $X$ with $\partial A \subset F$ and $\partial A \cap \beta=\emptyset$ is boundary parallel.

Proof. (1) Let $E$ be the disk in $X$ bounded by $\alpha \times 1$, cutting $X$ into $X^{\prime}=T \times I$. Note that $X$ is a compression body, and $\{E\}$ is the unique (up to isotopy) complete disk system for $X$. 
By assumption $\alpha \cup \beta$ cuts $F$ into disks, hence $|\alpha \cap \beta| \geq 3$. Since $\alpha$ intersects $\beta$ minimally, we may choose a hyperbolic structure on the surface $F$ so that $\alpha, \beta$ are geodesics. Let $D$ be a compressing disk for $F$ in $X$. Up to isotopy we may assume that $\gamma=\partial D$ is a geodesic or a slight push off of a geodesic if it is isotopic to $\alpha$ or $\beta$. Then both $|\gamma \cap \alpha|$ and $|\gamma \cap \beta|$ are minimal up to isotopy.

We may assume that $D \cap E$ consists of arcs. If $D \cap E \neq \emptyset$, by taking an arc that is outermost on $D$, surgering $E$ along the corresponding outermost disk in $D$, and discarding one of the resulting components, we get a new disk $E^{\prime}$ having fewer intersections with $D$, such that $\left\{E^{\prime}\right\}$ is a complete disk system for $X$. Since $\{E\}$ is the unique complete disk system for $X, E^{\prime}$ is isotopic to $E$. Since $\left|\partial E^{\prime} \cap \partial D\right|<|\partial E \cap \partial D|=|\alpha \cap \gamma|$ and $\partial E^{\prime}$ is isotopic to $\partial E$, this is a contradiction to the fact that $|\gamma \cap \alpha|$ is minimal up to isotopy. Therefore $D \cap E=\emptyset$. Hence $D$ either (a) is parallel to $E$, or (b) cuts off a solid torus containing $E$.

In case (a) $\partial D$ is a parallel copy of $\alpha$, so $|\partial D \cap \beta|=|\alpha \cap \beta| \geq 3$ and we are done. In case (b), let $F_{1}$ be the punctured torus on $F$ bounded by $\gamma$ which does not contain $\alpha$. If $|\gamma \cap \beta| \leq 2$ then $F_{1}$ contains at most one $\operatorname{arc}$ of $\beta$, so it contains an essential loop disjoint from $\alpha \cup \beta$, which is a contradiction to the assumption that $\alpha \cup \beta$ cuts $F$ into disks.

(2) Let $A$ be an incompressible annulus in $X$ with $\partial A \subset F-\beta$. We may assume that $\alpha, \beta$ are hyperbolic geodesics, and each component of $\partial A$ is either a geodesic, or a slight push off of a geodesic if it is parallel to $\alpha, \beta$ or another component of $\partial A$. Thus both $|\partial A \cap \alpha|$ and $|\partial A \cap \beta|$ are minimal; in particular, $\partial A \cap \beta=\emptyset$. As in (1), this implies that $A \cap E$ consists of essential $\operatorname{arcs}$ on $A$. If $A \cap E=\emptyset$ then $\partial A$ lies in $F \mid(\alpha \cup \beta)$, but since $A$ is incompressible while each component of $F \mid(\alpha \cup \beta)$ is a disk, this is impossible. Therefore we may assume that $A \cap E$ is a non-empty set $C$ of essential arcs on $A$.

Let $B_{i}$ be a component of $A \mid C$. Then $B_{i}$ is a disk in $X^{\prime}=T \times I$, so $\partial B_{i}$ is a trivial loop on $T^{\prime}$, bounding a disk $B_{i}^{\prime}$ on $T^{\prime}$. Let $E_{1}, E_{2}$ be the two copies of $E$ on $T^{\prime}$. If $B_{i}^{\prime} \cap\left(E_{1} \cup E_{2}\right)$ is a single disk then one can use a disk component of $B_{i}^{\prime} \cap F$ to isotope $A$ to reduce $|\partial A \cap \partial E|=|\partial A \cap \alpha|$, which is a contradiction to the minimality of $|\partial A \cap \alpha|$. Therefore $B_{i}^{\prime} \cap\left(E_{1} \cup E_{2}\right)$ consists of two disks, and $B^{\prime} \cap F$ is a single disk $P_{i}$. One can check that $\cup P_{i}$ is an annulus on $F$ parallel to $A$.

Lemma 23.13 The manifold $M_{14}$ is hyperbolic.

Proof. Cutting $M_{14}$ along the surface $F_{b}$, we obtain two manifolds $X_{1}, X_{2}$, where $X_{1}$ is the one containing the four bigon faces of $F_{a}$, and $X_{2}$ contains the two 4-gon faces of $F_{a}$. Let $\sigma_{1}, \sigma_{2}$ be the bigons on $F_{a}$ bounded by the edges $E \cup F$ and $B \cup Y$ respectively in Figure 20.6, and let $\sigma_{3}$ be the 4-gon bounded by the edges $B \cup C \cup Y \cup F$. Note that any other face of $F_{a}$ is parallel in $X_{i}$ to one of these.

Let $A_{i}=X_{i} \cap T_{0}$. Then $X_{1}$ is obtained from the genus 2 surface $F_{b} \cup A_{1}$ by attaching $\sigma_{1}, \sigma_{2}$ and then a 3 -cell, hence it is a handlebody of genus 2 because $\partial \sigma_{1}, \partial \sigma_{2}$ are disjoint nonparallel nonseparating curves on $F_{b} \cup A_{1}$. The core of 
$A_{1}$ is a curve on $\partial X_{1}$ such that after attaching a 2-handle to $X_{1}$ along $A_{1}$ we get the manifold on the side of $\hat{F}_{b}$ which contains no torus boundary component, hence from Figure 22.14(b) we see that it is the double branched cover of a Montesinos tangle $T(2,2)$, which is a twisted $I$-bundle over the Klein bottle. This implies that the surface $F_{b}=\partial X_{1}-A_{1}$ is incompressible in $X_{1}$.

Now consider $X_{2}$. Let $F$ be the genus 2 surface $F_{b} \cup A_{2}, \alpha$ the boundary of $\sigma_{3}$, and $\beta$ the core of $A_{2}$. Then $\alpha$ intersects $\beta$ minimally at four points. From Figure 20.6(b) we see that the edges $B, C, Y, F$ cut the surface $F_{b}$ into two disks, hence $\alpha \cup \beta$ cuts $F$ into disks. $X_{2}$ is obtained from $F \times I$ by attaching a 2-handle along the curve $\alpha \times\{0\}$. Therefore it satisfies the conditions of Lemma 23.12. In particular, $F_{b}$ is incompressible in $X_{2}$.

Since $X_{1}$ is a handlebody and $X_{2}$ is a compression body, they are irreducible and atoroidal. Since $M_{14}$ is obtained by gluing $X_{1}, X_{2}$ along the incompressible surface $F_{b}, M_{14}$ is also irreducible. It is well known that an incompressible surface in a Seifert fiber space is either vertical, and therefore an annulus or torus, or horizontal, in which case it intersects all boundary components. Since the surface $F_{b}$ satisfies neither condition, we see that $M_{14}$ is not Seifert fibered. It remains to show that $M_{14}$ is atoroidal.

Assume $M_{14}$ is toroidal and let $T_{1}$ be an essential torus in $M_{14}$ intersecting $F_{b}$ minimally. Since $X_{i}$ is atoroidal, $T_{1}$ intersects $X_{i}$ in incompressible annuli. A component $A_{2}^{\prime}$ of $T_{1} \cap X_{2}$ is an incompressible annulus in $X_{2}$ disjoint from $\beta$, hence by Lemma 23.12 it is parallel to an annulus $A^{\prime \prime}$ on $\partial X_{2}$. If $A^{\prime \prime} \subset F_{b}$ then $T_{1}$ can be isotoped to reduce $\left|T_{1} \cap F_{b}\right|$, which is a contradiction to the minimality assumption. Therefore $A^{\prime \prime} \supset \beta$ and hence $A^{\prime \prime} \supset A_{2}$, so each component of $\partial A^{\prime \prime}$ is parallel to a component of $\partial F_{b}$. Since this is true for all components of $T_{1} \cap X_{2}$, we see that each component of $T_{1} \cap F_{b}$ is parallel to a component of $\partial F_{b}$.

Now let $A_{1}^{\prime}$ be a component of $T_{1} \cap X_{1}$. By the above, the two boundary components of $A_{1}^{\prime}$ are parallel on $\partial X_{1}$. Since $X_{1}$ is a handlebody, $A_{1}^{\prime}$ is parallel to an annulus $A_{2}^{\prime \prime}$ on $\partial X_{1}$. For the same reason as above, it must contain the annulus $A_{1}$. This is true for all components of $T_{1} \cap X_{1}$. Let $A_{i}^{\prime}$ be a component of $T_{1} \cap X_{i}$ which is closest to $A_{i}$. Then $\partial A_{1}^{\prime}=\partial A_{2}^{\prime}$, hence $T_{1}=A_{1}^{\prime} \cup A_{2}^{\prime}$. It follows that $T_{1}$ is parallel to $T_{0}$, contradicting the assumption that $T_{1}$ is essential in $M_{14}$.

Theorem 23.14 The manifolds $M_{i}$ in Definition 21.3 are all hyperbolic.

Proof. This follows from [GW1, Theorem 1.1] for $i=1,2,3$, and from Lemmas 23.7, 23.8, 23.10, 23.11 and 23.13 for $i>3$.

\section{Toroidal surgery on knots in $S^{3}$}

Recall that each of the manifolds $M_{1}, M_{2}, M_{3}$ admits two toroidal Dehn fillings $r_{i}^{\prime}, r_{i}^{\prime \prime}$ on a torus boundary component $T_{0}$ with distance 4 or 5 . These are the exteriors of the links $L_{1}, L_{2}, L_{3}$ in Figure 24.1. Let $L_{i}=K_{i}^{\prime} \cup K_{i}^{\prime \prime}$, where $K_{i}^{\prime}$ is the left component of $L_{i}$. Let $T_{1}=\partial N\left(K_{i}^{\prime}\right)$, and $T_{0}=\partial N\left(K_{i}^{\prime \prime}\right)$. 


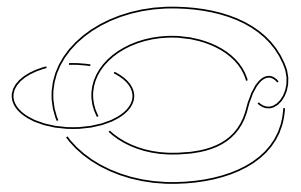

(1)

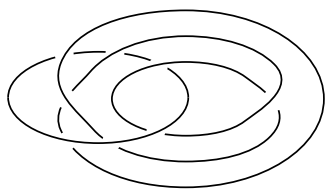

(2)

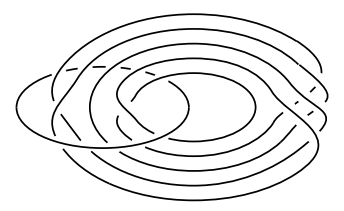

(3)

Figure 24.1

Each $M_{i}$ has a pair of toroidal slopes $r_{i}^{\prime}, r_{i}^{\prime \prime}$ on $T_{0}$. These are given in [GW1, Theorem 7.5] and shown in Figures 7.2, 7.4 and 7.5 of [GW1].

Lemma 24.1 With respect to the preferred meridian-longitude pair of $K_{i}^{\prime \prime}$, the slopes $r_{i}^{\prime}, r_{i}^{\prime \prime}$ are given as follows, up to relabeling.

(1) $r_{1}^{\prime}=0$ and $r_{1}^{\prime \prime}=4$

(2) $r_{2}^{\prime}=-2$ and $r_{2}^{\prime \prime}=2$.

(3) $r_{3}^{\prime}=-9$ and $r_{3}^{\prime \prime}=-23 / 2$.

Proof. (1) This is basically proved in [GW1, Lemma 7.1]. It was shown that $M_{1}$ is the double branched cover of the tangle $Q_{1}$ in Figure 7.2(c) of [GW1]. Let $m$ be the meridian, $l$ the preferred longitude, and $l^{\prime}$ the blackboard longitude of the diagram of $K_{1}^{\prime \prime}$ in [GW1, Figure 7.2(a)]. Calculating the linking number of $l^{\prime}$ with $K_{1}^{\prime \prime}$ in Figure 7.2(a) we see that $l^{\prime}=2 m+l$. Let $\eta: M_{1} \rightarrow Q_{1}$ be the branched covering map. If $r$ is a slope on $T_{0}$ then $\eta(r)$ is a curve of a certain slope on the inside boundary sphere, which will be denoted by a number in $\mathbb{Q}_{0}=\mathbb{Q} \cup\{\infty\}$. One can check that $\eta(m)=0 / 1$, and $\eta\left(l^{\prime}\right)=1 / 0$. The two toroidal slopes $r_{1}^{\prime}, r_{1}^{\prime \prime}$ map to slopes $-1 / 2$ and $1 / 2$, as shown in Figure $7.2(\mathrm{~d})$ and (e) of [GW1]. We have $\varphi\left(-2 m+l^{\prime}\right)=(-2 \times 0+1) /(-2 \times 1+0)=-1 / 2$, and $\varphi\left(2 m+l^{\prime}\right)=(2 \times 0+1) /(2 \times 1+0)=1 / 2$. Therefore $r_{1}^{\prime}=-2 m+l^{\prime}=$ $-2 m+(2 m+l)=l$ and $r_{1}^{\prime \prime}=2 m+l^{\prime}=4 m+l$.

(2) This is similar to (1), using [GW1, Figure 7.4] instead. We have $\eta(m)=$ $0 / 1, \eta\left(l^{\prime}\right)=1 / 2, \eta\left(r_{2}^{\prime}\right)=1 / 0, \eta\left(r_{2}^{\prime \prime}\right)=1 / 4$, and $l=l^{\prime}$. Therefore $r_{2}^{\prime}=-2 m+l$ and $r_{2}^{\prime \prime}=2 m+l$.

(3) Use 7.5(k) to denote [GW1, Figure 7.5(k)]. 7.5(a) shows that $l^{\prime}=l-6 m$. A careful tracking of $l^{\prime}$ during the modification from 7.5(a) to 7.5(b) then to 7.5(c) shows that $\eta(m)=0 / 1$ and $\eta\left(l^{\prime}\right)=1 / 3$. From 7.5(c) and 7.5(e) we see that $\eta\left(r_{3}^{\prime}\right)=1 / 0$ and $\eta\left(r_{3}^{\prime \prime}\right)=2 / 5$. Therefore $r_{3}^{\prime}=-3 m+l^{\prime}=-9 m+l$, and $r_{3}^{\prime \prime}=-m+2 l^{\prime}=-m+2(l-6 m)=-13 m+2 l$.

Lemma 24.2 Suppose $K$ is a hyperbolic knot in $S^{3}$ admitting two toroidal Dehn surgeries $K\left(r_{1}\right), K\left(r_{2}\right)$ with $\Delta\left(r_{1}, r_{2}\right)=4$ or 5 . Then there is an $i \in\{1,2,3\}$ and a slope $s$ on $T_{1}$ of $M_{i}$ such that $\left(E(K), r_{1}, r_{2}\right) \cong\left(M_{i}(s), r_{i}^{\prime}, r_{i}^{\prime \prime}\right)$.

Proof. Let $F_{a}$ be an essential punctured torus in $M_{K}=S^{3}-\operatorname{Int} N(K)$ such that $\hat{F}_{a}$ is an essential torus in $K\left(r_{a}\right)$, chosen so that $\left|\partial F_{a}\right|$ is minimal. By 
Theorem 21.4 the triple $\left(E(K), r_{1}, r_{2}\right)$ is equivalent to either $\left(M_{i}, r_{i}^{\prime}, r_{i}^{\prime \prime}\right)$ with $1 \leq i \leq 14$, or to $\left(M_{i}(s), r_{i}^{\prime}, r_{i}^{\prime \prime}\right)$ for some $i=1,2,3,14$. Therefore we need only show that the manifold $M_{i}(i=4, \ldots, 14)$ is not the exterior of a knot or link in $S^{3}$.

When $i=4$, the surface $F_{b}$ has two boundary circles on $T_{0}$ with the same orientation. Let $A$ be an annulus on $T_{0}$ connecting these two boundary components. Then $F_{b} \cup A$ is a non-orientable closed surface in $M_{4}$. It follows that $M_{4}$ cannot be the exterior of a knot in $S^{3}$ because $S^{3}$ contains no embedded non-orientable surface.

For $i=5$, let $V_{b}$ be the Dehn filling solid torus of $M_{5}\left(r_{b}\right)$. Then the $\mathbb{Z}_{2}$ homology group $H$ of $V_{b} \cup F_{b}$ is generated by $\alpha, x$ and $y$, where $\alpha$ is the core of $V_{b}$, and $x, y$ are represented by the edges $A$ and $E$ in Figure 11.10(b), respectively. A bigon in Figure 11.10(a) gives the relation $x=y$. Consider the quotient group $H^{\prime}$ obtained from $H$ by identifying $x$ with $y$. Then $H^{\prime}=\mathbb{Z}_{2} \oplus \mathbb{Z}_{2}$ is generated by $\alpha$ and $x$. Each corner of Figure 11.10(a) represents the element $\alpha$, and each edge represents $x$ in $H^{\prime}$. Since each face in Figure 11.10(a) has an even number of edges and an even number of corners on its boundary, it represents 0 in $H^{\prime}$. Therefore

$$
H_{1}\left(M_{5}\left(r_{b}\right), \mathbb{Z}_{2}\right)=H_{1}\left(V_{b} \cup F_{b} \cup F_{a}, \mathbb{Z}_{2}\right)=H^{\prime}=\mathbb{Z}_{2} \oplus \mathbb{Z}_{2} .
$$

Since the $\mathbb{Z}_{2}$ homology of any manifold obtained by Dehn surgery on a knot in $S^{3}$ is either trivial or $\mathbb{Z}_{2}$, it follows that $M_{5}$ is not a knot exterior.

Now assume that $M_{i}$ is the exterior of a knot $K$ in $S^{3}$ for some $i \geq 6$. By Theorem $23.14 K$ is hyperbolic. Put $\mathbb{Q}_{0}=\mathbb{Q} \cup\{\infty\}$. A number in $\mathbb{Q}_{0}$ is represented by $p / q$, where $p, q$ are coprime integers, and $q \geq 0$. Given a meridian-longitude pair $(m, l)$ and $r=p / q \in \mathbb{Q}_{0}$, denote by $K(r)$ the manifold obtained by surgery on $K$ along the slope $p m+q l$. There is a one to one correspondence $\eta: \mathbb{Q}_{0} \rightarrow \mathbb{Q}_{0}$ such that $\Delta(\eta(r), \eta(s))=\Delta(r, s)$, and $K(\eta(r))$ is the double branched cover of $Q_{i}(r)$, which is the manifold $X_{i}(r)$ given in Lemma 22.2. Since $K\left(\eta\left(r_{3}\right)\right)$ is a lens space, by the Cyclic Surgery Theorem [CGLS, p.237] the slope $\eta\left(r_{3}\right)$ is an integer slope with respect to the preferred meridian-longitude of $K$. To simplify the calculation, let $l=\eta\left(r_{3}\right)$.

By [GLu, Theorem 1.1] $\eta\left(r_{1}\right)$ and $\eta\left(r_{2}\right)$ are integer or half integer slopes. Suppose $\eta\left(r_{i}\right)=p_{i} / q_{i}$. Then $p_{3} / q_{3}=0 / 1$. By the above we have $q_{i}=1$ or 2 for $i=1,2$. By Lemma $22.2,\left|p_{1}\right|=\Delta\left(r_{1}, r_{3}\right)=1$, and $\left|p_{2}\right|=\Delta\left(r_{2}, r_{3}\right) \leq 2$.

If $\left|p_{2}\right|=1$ then $4 \leq \Delta\left(r_{1}, r_{2}\right)=\Delta\left(\eta\left(r_{1}\right), \eta\left(r_{2}\right)\right)=\left|p_{1} q_{2}-p_{2} q_{1}\right|$ implies that $q_{1}=q_{2}=2$. This is a contradiction to [GWZ, Theorem 1], which says that a hyperbolic knot in $S^{3}$ admits at most one non-integral toroidal surgery.

We now have $\left|p_{2}\right|=2$, so $\eta\left(r_{2}\right)=p_{2} / q_{2}= \pm 2 / 1$. Since $\Delta\left(r_{1}, r_{2}\right)=\mid p_{1} q_{2}-$ $p_{2} q_{1}|=| \pm 1-( \pm 2) q_{1} \mid \geq 4$, we must have $p_{1} / q_{1}=\mp 1 / 2$, and $\Delta=5$. From Lemma 22.2 we see that for $i \in\{6, \ldots, 13\}$, the only $M_{i}$ satisfying $\Delta\left(r_{2}, r_{3}\right)=2$ and $\Delta\left(r_{1}, r_{2}\right)=5$ are the ones with $i=7,10$ or 11 .

Consider the case $i=10$. Let $r_{0}$ be the slope such that $\eta\left(r_{0}\right)$ is the meridian slope $1 / 0$. Then we have $\Delta\left(r_{0}, r_{i}\right)=\Delta\left(\eta\left(r_{0}\right), \eta\left(r_{i}\right)\right)=\Delta\left(1 / 0, p_{i} / q_{i}\right)=q_{i}$. Therefore by the above we have $\Delta\left(r_{0}, r_{i}\right)=q_{i}=2,1,1$ for $i=1,2,3$, respectively. By Lemma 22.2 we have $r_{1}=0 / 1, r_{2}=-5 / 2$ and $r_{3}=1 / 0$. Let 
$r_{0}=p^{\prime} / q^{\prime}$. Then we have

$$
\begin{aligned}
& \Delta\left(r_{0}, r_{1}\right)=\left|p^{\prime}\right|=2 \\
& \Delta\left(r_{0}, r_{2}\right)=\left|2 p^{\prime}+5 q^{\prime}\right|=1 \\
& \Delta\left(r_{0}, r_{3}\right)=q^{\prime}=1
\end{aligned}
$$

These equations have a unique solution $r_{0}=-2 / 1$. One can check that $Q_{10}(-2 / 1)$ is the 2-bridge knot $K_{2 / 7}$, so its double branched cover is $L(7,2) \neq S^{3}$, which is a contradiction.

The tangles $Q_{7}, Q_{11}$ and $Q_{14}$ in Figures 22.7, 22.11 and 22.14 have a circle component. If $M_{i}$ is a knot exterior in $S^{3}$ then there is a slope $r$ such that the double branched cover of $Q_{i}(r)$ is $S^{3}$. Since each of $Q_{7}(r)$ and $Q_{11}(r)$ has at least two components, its double branched cover has nontrivial $\mathbb{Z}_{2}$ homology [Sa, Sublemma 15.4], so $M_{7}$ and $M_{11}$ are not knot exteriors in $S^{3}$. Similarly $M_{14}$ is not the exterior of a link in $S^{3}$.

Lemma 24.3 (1) Let $i \in\{1,2,3\}$. If $r_{1}, r_{2}$ are toroidal slopes of $M_{i}$ on $T_{0}$ with $\Delta\left(r_{1}, r_{2}\right) \geq 4$, then $\left\{r_{1}, r_{2}\right\}=\left\{r_{i}^{\prime}, r_{i}^{\prime \prime}\right\}$.

(2) The slope -7 is a solid torus filling slope on $T_{0}$ of $M_{3}$, and there is an orientation preserving homeomorphism of $M_{3}$ which interchanges the two solid torus filling slopes $\{1 / 0,-7 / 1\}$ and the two toroidal slopes $\{-9,-13 / 2\}$.

Proof. Since $M_{1}, M_{2}, M_{3}, M_{14}$ are the only ones in Definition 21.3 with two boundary components, by Theorem $21.4\left(M_{i}, r_{1}, r_{2}\right)$ is equivalent to one of the $\left(M_{j}, r_{j}^{\prime}, r_{j}^{\prime \prime}\right)$ with $j=1,2,3,14$. Since $M_{1}, M_{2}, M_{3}$ are link complements in $S^{3}$ and by Lemma $24.2 M_{14}$ is not, we have $j \neq 14$. Computing $H_{1}\left(M_{j}, T_{1}\right)$ shows $H_{1}\left(M_{1}, T_{0}\right)=\mathbb{Z}, H_{1}\left(M_{2}, T_{0}\right)=\mathbb{Z}_{3}$, and $H_{1}\left(M_{1}, T_{0}\right)=\mathbb{Z}_{5}$, hence we must have $j=i$.

By definition there is a homeomorphism $\varphi:\left(M_{i}, r_{1}, r_{2}\right) \rightarrow\left(M_{i}, r_{i}^{\prime}, r_{i}^{\prime \prime}\right)$, up to relabeling of $r_{1}, r_{2}$. For $i=1,2$, by [Ga1] and [Be] the knot $K_{i}^{\prime \prime}$ has no nontrivial solid torus surgery, hence $\varphi(m)=m$, where $m$ is a meridian of $K_{i}^{\prime \prime}$. Since $\Delta\left(m, r_{i}^{\prime}\right)=\Delta\left(m, r_{i}^{\prime \prime}\right)=1$, by the homeomorphism we also have $\Delta\left(m, r_{1}\right)=\Delta\left(m, r_{2}\right)=1$, so $r_{1}, r_{2}$ are also integer slopes. It follows that if $\left\{r_{1}, r_{2}\right\} \neq\left\{r_{1}^{\prime}, r_{1}^{\prime \prime}\right\}$ then there is a pair of toroidal slopes with distance at least 5. Since $M_{1}, M_{2}$ is not homeomorphic to $M_{3}$, this is a contradiction to Theorem 21.4 and $[\mathrm{Go}]$.

Now suppose $i=3$. By an isotopy one can deform the tangle in [GW1, Figure 7.5(c)], which is shown in Figure 24.2(a), to the one in Figure 24.2(b), which is invariant under the $\pi$ rotation $\psi$ along the forward slash diagonal. The 1/0 slope in Figure 24.2(b) corresponds to the 1/2 slope in Figure 24.2(a), which, by the proof of Lemma 24.1(3), lifts to the slope $-7 m+l$ on $T_{0}$. The two toroidal slopes $1 / 0$ and $5 / 2$ for the tangle in Figure 22.2(a) correspond to the slopes $-1 / 2$ and 2 in Figure 24.2(b), which are interchanged by $\psi$. It follows that $\psi$ lifts to an orientation preserving homeomorphism $\psi^{\prime}: M_{3} \rightarrow M_{3}$, which interchanges the two solid torus filling slopes $\{1 / 0,-7 / 1\}$ and the two toroidal 
slopes $\{-9,-13 / 2\}$. In fact, $\psi^{\prime}$ is represented by the matrix

$$
A=\left(\begin{array}{rr}
7 & 1 \\
-1 & 0
\end{array}\right)
$$

in the sense that if $A(p, q)^{t}=\left(p^{\prime}, q^{\prime}\right)^{t}$ (where $B^{t}$ denotes the transpose of the matrix $B)$ then $\psi^{\prime}(p m+q l)=p^{\prime} m+q^{\prime} l$.

Solid torus surgeries on knots in a solid torus have been completely classified by Gabai [Ga1] and Berge [Be]. It was shown that there is only one knot admitting two nontrivial solid torus surgeries, which is a 7 -braid. Since $K_{3}^{\prime \prime}$ is a 5-braid, we see that $m=1 / 0$ and $m^{\prime}=-7 / 1$ are the only solid torus filling slopes on $T_{0}$. Therefore the homeomorphism $\varphi:\left(M_{3}, r_{3}^{\prime}, r_{3}^{\prime \prime}\right) \rightarrow\left(M_{3}, r_{1}, r_{2}\right)$ must map the set of two curves $\left\{m, m^{\prime}\right\}$ to itself, possibly with the orientation of one or both of the curves reversed. If $\varphi$ preserves the orientation of $m^{\prime}$ and reverses the orientation of $m$ then $\varphi\left(r_{3}^{\prime}\right)=9 / 1$ would also be a toroidal slope, which is a contradiction to [Go] because $\Delta(-9 / 1,9 / 1)=18>8$. Similarly $\varphi$ cannot preserve the orientation of $m$ while reversing the orientation of $m^{\prime}$. Therefore $\varphi$ is orientation preserving and its induced map on the set of slopes on $T_{0}$ is either the identity map, which fixes $\left\{r_{3}^{\prime}, r_{3}^{\prime \prime}\right\}$, or the same as that induced by $\psi^{\prime}$ above, which interchanges $\left\{r_{3}^{\prime}, r_{3}^{\prime \prime}\right\}$.

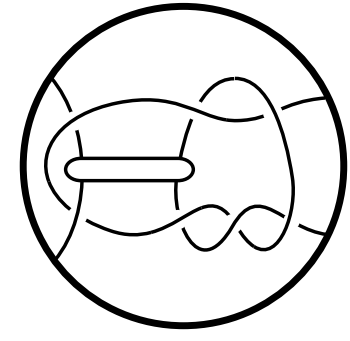

(a)

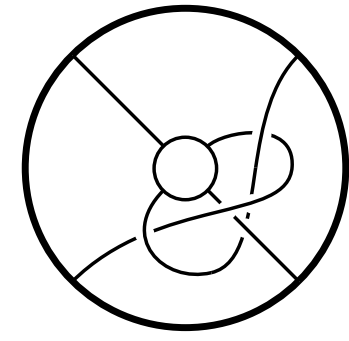

(b)

Figure 24.2

Theorem 24.4 Suppose $K$ is a hyperbolic knot in $S^{3}$ admitting two toroidal surgeries $K\left(r_{1}\right), K\left(r_{2}\right)$ with $\Delta\left(r_{1}, r_{2}\right) \geq 4$. Then $\left(K, r_{1}, r_{2}\right)$ is equivalent to one of the following, where $n$ is an integer.

(1) $K=L_{1}(n), r_{1}=0, r_{2}=4$.

(2) $K=L_{2}(n), r_{1}=2-9 n, r_{2}=-2-9 n$.

(3) $K=L_{3}(n), r_{1}=-9-25 n, r_{2}=-(13 / 2)-25 n$.

(4) $K$ is the Figure 8 knot, $r_{1}=4, r_{2}=-4$.

Proof. By [Go] the Figure 8 knot $L_{1}(-1)$ is the only hyperbolic knot in $S^{3}$ admitting two toroidal surgeries of distance at least 6 , so we assume $\Delta=4$ or 5 . By Lemma 24.2 there is a homeomorphism $\varphi:\left(M_{i}(s),\left\{r_{i}^{\prime}, r_{i}^{\prime \prime}\right\}\right) \cong\left(E(K),\left\{r_{1}, r_{2}\right\}\right)$ 
for some $i=1,2,3$ and $s \subset T_{1}$. We only need to show that $s=1 / n$ because the slopes $r_{i}$ can then be calculated using Lemma 24.1 and the Kirby calculus [Ro, p.267].

If Dehn filling on $T_{1}$ of $\partial M_{i}$ along slope $s$ produces a knot exterior $E(K)=$ $M_{i}(s)$, then the meridian-longitude of $K$ may be different from that of $K_{i}^{\prime \prime}$ on $T_{0}$. We use $\left(m^{\prime \prime}, l^{\prime \prime}\right)$ (resp. $\left.(m, l)\right)$ to denote a meridian-longitude pair of $K_{i}^{\prime \prime}$ (resp. $K$ ) in $S^{3}$.

Claim 1. If $E(K)=M_{1}(s)$ for some $s$ on $T_{1}$ of $\partial M_{1}$ then $s=1 / n$.

Since the linking number between the two components of $L_{1}$ is 0 , a $p / q$ Dehn filling on $T_{1}$ produces a manifold $M_{1}(p / q)$ with $H_{1}\left(M_{1}(p / q), \mathbb{Z}\right)=\mathbb{Z} \oplus \mathbb{Z}_{p}$, hence $M_{1}(p / q)$ is a knot complement only if $|p|=1$. It follows that $K=L_{1}(n)$, where $n=q p$.

Claim 2. If $E(K)=M_{2}(s)$ for some $s$ on $T_{1}$ of $\partial M_{2}$ then $s=1 / n$ for some $n$.

As before, let $L_{2}=K_{i}^{\prime} \cup K_{i}^{\prime \prime}$. Let $M=E(K)$. Assume $s=p / q$ and $|p|>1$. We have $K\left(m^{\prime \prime}\right)=L_{2}\left(s, m^{\prime \prime}\right)=K_{i}^{\prime}(s)=L(p, q)$. Therefore by the Cyclic Surgery Theorem [CGLS], $m^{\prime \prime}$ is an integer slope with respect to $(m, l)$, say $m^{\prime \prime}=a m+l$. By [GLu] the toroidal slopes $r_{1}, r_{2}$ of $K$ are integer or half integer slopes with respect to $(m, l)$. Recall that $\varphi\left(r_{2}^{\prime}\right)=r_{1}$ and $\varphi\left(r_{2}^{\prime \prime}\right)=r_{2}$. Since $m^{\prime \prime}$ is an integer slope, $r_{1}, r_{2}$ cannot both be integer slopes, otherwise $4=\Delta\left(r_{1}, r_{2}\right) \leq \Delta\left(r_{1}, m^{\prime \prime}\right)+\Delta\left(m^{\prime \prime}, r_{2}\right)=2$, which is a contradiction. Also by [GWZ] they cannot both be half integer slopes.

Now assume $r_{1}$ is an integer slope and $r_{2}$ is a half integer slope with respect to $(m, l)$. Since $m^{\prime \prime}$ is an integer slope, we may choose $l=m^{\prime \prime}$. Then $r_{1}=p_{1} m+l$ and $r_{2}=p_{2} m+2 l$, so $\Delta\left(r_{1}, m^{\prime \prime}\right)=\Delta\left(r_{2}, m^{\prime \prime}\right)=1$ implies $p_{1}, p_{2}= \pm 1$. But then $\Delta\left(r_{1}, r_{2}\right)=\left|2 p_{1}-p_{2}\right| \leq 3$, a contradiction.

Claim 3. If $E(K)=M_{3}(s)$ then there is an integer $n$ and a homeomorphism $\eta:\left(M_{3}(1 / n),\left\{r_{3}^{\prime}, r_{3}^{\prime \prime}\right\}\right) \rightarrow\left(E(K),\left\{r_{1}, r_{2}\right\}\right)$.

By Lemma $24.3 M_{3}(-7)$ is a solid torus, and the meridian slope $m^{\prime \prime}$ and the slope $r=-7$ are the only solid torus filling slopes on $T_{0}$. If $\varphi\left(m^{\prime \prime}\right)=m$ then $S^{3}=K\left(m^{\prime \prime}\right)=K_{i}^{\prime}(s)$ implies that $s=1 / n$ for some $n$, so $\eta=\varphi$ is the required map. If $\varphi(r)=m$, let $\psi$ be the orientation preserving homeomorphism of $M_{3}$ given in Lemma 24.3, which maps $m^{\prime \prime}$ to $r$. By Lemma $24.3 \psi$ interchanges the slopes $r_{3}^{\prime}, r_{3}^{\prime \prime}$. Let $s^{\prime}=\psi^{-1}(s)$. Then $\varphi \circ \psi:\left(M_{3}\left(s^{\prime}\right), r_{3}^{\prime \prime}, r_{3}^{\prime}\right) \cong\left(E(K), r_{1}, r_{2}\right)$ maps $m^{\prime \prime}$ to $m$. As above this implies that $s^{\prime}=1 / n$, hence $\eta=\varphi \circ \psi$ is the required map.

We now assume that $\varphi\left(m^{\prime \prime}\right) \neq m$ and $\varphi(r) \neq m$. Note that $K\left(m^{\prime \prime}\right)$ and $K(r)$ can be obtained from the solid tori $M_{3}\left(m^{\prime \prime}\right)$ and $M_{3}(r)$ by $s$ filling on $T_{1}$, so they have cyclic $\pi_{1}$, hence by [CGLS] $r, m^{\prime \prime}$ are integer slopes of $K$. Choose $l=m^{\prime \prime}$. Since $\Delta\left(m^{\prime \prime}, r\right)=1$, we may assume $r=1 / 1$ up to rechoosing the orientation of $l$. The toroidal slopes $r_{3}^{\prime}=-9$ satisfy $\Delta\left(r_{3}^{\prime}, m^{\prime \prime}\right)=1$ and $\Delta\left(r_{3}^{\prime}, r\right)=2$, which implies $r_{3}^{\prime}=-1 / 1$ or $1 / 3$ with respect to $(m, l)$. The second is impossible by [GLu]. Similarly the fact that $\Delta\left(r_{3}^{\prime \prime}, m^{\prime \prime}\right)=2$ and $\Delta\left(r_{3}^{\prime \prime}, r\right)=1$ implies that 
$r_{3}^{\prime \prime}=2$ with respect to $(m, l)$. But then we have $5=\Delta\left(r_{3}^{\prime}, r_{3}^{\prime \prime}\right)=\Delta(-1,2)=3$, a contradiction.

Corollary 24.5 A hyperbolic knot $K$ in $S^{3}$ has at most four toroidal surgeries. If there are four, then they are consecutive integers.

Proof. By [GLu] a toroidal slope of $K$ must be integer or half integer, and if it is a half integer then $K$ is a Eudave-Muñoz knot. By [T1, Corollary 1.2], if $K$ is a Eudave-Muñoz knot then it has at most three toroidal slopes, hence the result is true if $K$ has a half integer toroidal slope. Therefore we may assume that all toroidal slopes of $K$ are integer slopes. The result follows if $\Delta(r, s)<4$ for all pairs of toroidal slopes $(r, s)$ of $K$. Therefore by Theorem 24.4 we need only show that if $K$ is either $L_{1}(n)$ or $L_{2}(n)$ for some $n$ then $K$ has at most three integer toroidal slopes.

If $K$ is the knot $L_{1}(n)$ in Theorem 24.4(1) then by [BW] it has exactly two toroidal slopes unless it is the Figure 8 knot, which has three toroidal slopes.

Now consider a knot $K=L_{2}(n)$ in Theorem 24.4(2) and let $r$ be an integral toroidal slope of $K$ other than $r_{1}, r_{2}$ in the Theorem. Since $\Delta\left(r_{i}, r\right) \leq$ 4, $r$ must be between $r_{1}$ and $r_{2}$. Denote by $M_{2}(p / q)$ the $p / q$ filling on $T_{0}$ with respect to the preferred meridian-longitude pair of $L_{2}$. By the proof of Lemma 24.1(2), $M_{2}(-1)$ is the double branched cover of $Q_{2}(1)$. Using the tangle in [GW1, Figure 7.4(c)] one can check that $Q_{2}(1)$ is a Montesinos tangle $T(1 / 2,-2 / 5)$, therefore $M_{2}(-1)$ is a small Seifert fiber space with orbifold $D^{2}(2,5)$. Since $L_{2}(n)(-1-9 n)$ is obtained from $M_{2}(-1)$ by Dehn filling on $T_{1}$ and contains no non-separating surface, it is atoroidal. Because of symmetry ( $L_{2}$ is amphicheiral), $M_{2}(1)$ is homeomorphic to $M_{2}(-1)$, so $L_{2}(n)(1-9 n)$ is also atoroidal. It follows that the only possible integer toroidal slopes of $L_{2}(n)$ are $j-9 n$ for $j=-2,0,2$. This completes the proof. (Actually it can be shown that $-9 n$ is not a toroidal slope of $L_{2}(n)$ either, so it has at most two integer toroidal slopes.)

The following corollary is an immediate consequence of Theorem 24.4.

Corollary 24.6 Let $K$ be a hyperbolic knot in $S^{3}$ which admits two toroidal surgeries along slopes $r_{1}, r_{2}$, and $\Delta=\Delta\left(r_{1}, r_{2}\right) \geq 4$. Then one of the $r_{i}$ is an integer, and the other one is an integer if $\Delta \neq 5$, and a half integer if $\Delta=5$.

Although there are infinitely many hyperbolic 3-manifolds $M$ with toroidal fillings $M(r), M(s)$ at distance 4 or 5 , we have shown that they all come from finitely many cores $X(r, s)$ as defined in Section 21 .

Question 24.7 Are there only finitely many cores $X(r, s)$ of toroidal Dehn fillings on hyperbolic 3-manifolds with $\Delta(r, s)=3$ ? $\Delta(r, s)=2$ ?

We observe that the answer to Question 24.7 in the case $\Delta(r, s)=1$ is almost certainly 'no'; here is an outline of an argument. Let $N$ be a closed irreducible 3 -manifold with a unique incompressible torus $T$ up to isotopy. Let $F$ be a oncepunctured torus, regarded as a disk with two bands. It is intuitively clear that, 
for any positive integer $n$, by tangling the bands in a sufficiently complicated fashion we can construct an embedding $F_{n}$ of $F$ in $N$ so that if $K_{n}=\partial F_{n}$, then $N-K_{n}$ is hyperbolic, and $K_{n}$ cannot be isotoped to meet $T$ in fewer than $n$ points. Let $M_{n}=N-\operatorname{Int} N\left(K_{n}\right)$, and let $r, s$ on $\partial M_{n}$ be the meridian of $K_{n}$ and the longitudinal slope defined by $F_{n}$, respectively. Then $\Delta(r, s)=1$, $M_{n}(r)=N$ is toroidal by definition, and $M_{n}(s)$ contains the non-separating torus $\hat{F}_{n}=F_{n} \cup D$, where $D$ is a meridian disk of $V_{s}$. Hence, if we make sure that $M_{n}(s)$ does not contain a non-separating sphere, then $M_{n}(s)$ is also toroidal. Since the number of intersections of $K_{n}$ with $T$ is at least $n$, the triples $\left(M_{n}, r, s\right)$ cannot all come from only finitely many cores.

\section{References}

[Be] J. Berge, The knots in $D^{2} \times S^{1}$ which have nontrivial Dehn surgeries that yield $D^{2} \times S^{1}$, Topology Appl. 38 (1991), 1-19.

[BW] M. Brittenham and Y-Q. Wu, The classification of exceptional Dehn surgeries on 2-bridge knots, Comm. Anal. Geom. 9 (2001), 97-113.

[CGLS] M. Culler, C. Gordon, J. Luecke and P. Shalen, Dehn surgery on knots, Annals Math. 125 (1987), 237-300.

[Eu] M. Eudave-Muñoz, Non-hyperbolic manifolds obtained by Dehn surgery on hyperbolic knots, Geometric Topology (Athens, GA, 1993), AMS/IP Stud. Adv. Math., 2.1, Amer. Math. Soc., Providence, RI, 1997, pp. $35-61$.

[Ga1] D. Gabai, Surgery on knots in solid tori, Topology 28 (1989), 1-6.

[Ga2] —, 1-bridge braids in solid tori, Top. Appl. 37 (1990), 221-235.

[Go] C. Gordon, Boundary slopes of punctured tori in 3-manifolds, Trans. Amer. Math. Soc. 350 (1998), 1713-1790.

[GLi] C. Gordon and R. Litherland, Incompressible planar surfaces in 3manifolds, Topology Appl. 18 (1984), 121-144.

[GLu] C. Gordon and J. Luecke, Non-integral toroidal Dehn surgeries, Comm. Anal. Geom. 12 (2004), 417-485.

[GW1] C. Gordon and Y-Q. Wu, Toroidal and annular Dehn fillings, Proc. London Math. Soc. 78 (1999), 662-700.

[GW2] —, Annular and boundary reducing Dehn surgery, Topology 39 (2000), 531-548.

[GW3] —, Annular Dehn fillings, Comment. Math. Helv. 75 (2000), 430456. 
[GWZ] C. Gordon, Y-Q. Wu and X. Zhang, Non-integral toroidal surgery on hyperbolic knots in $S^{3}$, Proc. Amer. Math. Soc. 128 (2000), 1869-1879.

[HM] C. Hayashi and K. Motegi, Only single twists on unknots can produce composite knots, Trans. Amer. Math. Soc. 349 (1997), 4465-4479.

[L1] S. Lee, Exceptional Dehn fillings on hyperbolic 3-manifolds with at least two boundary components, preprint.

[L2] —, Dehn fillings yielding Klein bottles, preprint.

[MaS] D. Matignon and N. Sayari, Klein slopes on hyperbolic 3-manifolds, preprint.

[MeS] W. Meeks III and P. Scott, Finite group actions on 3-manifolds, Invent. Math. 86 (1986), 287-346.

[Oh] S. Oh, Reducible and toroidal manifolds obtained by Dehn filling, Topology Appl. 75 (1997), 93-104.

[Ro] D. Rolfsen, Knots and Links Publish or Perish, 1990.

[Sa] M. Sakuma, Homology of abelian coverings of links and spatial graphs, Canadian J. Math. 47 (1995), 201-224.

[Sch] M. Scharlemann, Producing reducible 3-manifolds by surgery on a knot, Topology 29 (1990), 481-500.

[Sct] P. Scott, The geometry of 3-manifolds, Bull. London Math. Soc. 15 (1983), 401-487.

[T1] M. Teragaito, Distance between toroidal surgeries on hyperbolic knots in the 3-sphere, preprint, arXiv:math.GT/0312201

[T2] - Toroidal Dehn fillings on large hyperbolic 3-manifolds, preprint, arXiv:math.GT/0508250

[Wu1] Y-Q. Wu, Dehn fillings producing reducible manifolds and toroidal manifolds, Topology 37 (1998), 95-108.

[Wu2] - Incompressibility of surfaces in surgered 3-manifolds, Topology 31 (1992), 271-279.

[Wu3] —, Sutured manifold hierarchies, essential laminations, and Dehn surgery, J. Diff. Geom., 48 (1998), 407-437.

Dept. Mathematics, University of Texas at Austin, Austin, TX 78712

Email: gordon@math.utexas.edu

Department of Mathematics, University of Iowa, Iowa City, IA 52242

Email:wu@math.uiowa.edu 